Portland State University

PDXScholar

1988

\title{
A fractal analysis of diffusion limited aggregation
}

Cliff Myers

Portland State University

Follow this and additional works at: https://pdxscholar.library.pdx.edu/open_access_etds

Part of the Physics Commons

Let us know how access to this document benefits you.

\section{Recommended Citation}

Myers, Cliff, "A fractal analysis of diffusion limited aggregation" (1988). Dissertations and Theses. Paper 4047.

https://doi.org/10.15760/etd.5931

This Thesis is brought to you for free and open access. It has been accepted for inclusion in Dissertations and Theses by an authorized administrator of PDXScholar. Please contact us if we can make this document more accessible: pdxscholar@pdx.edu. 
AN ABSTRACT DF THE THESIS DF Cliff Myers for the Master of Science in Physics presented November 16, 1988.

Title: A Fractal Analysis of Diffusion Limited Aggregation. APPROVED BY MEMBERS OF THE THESIS COMMITTEE:

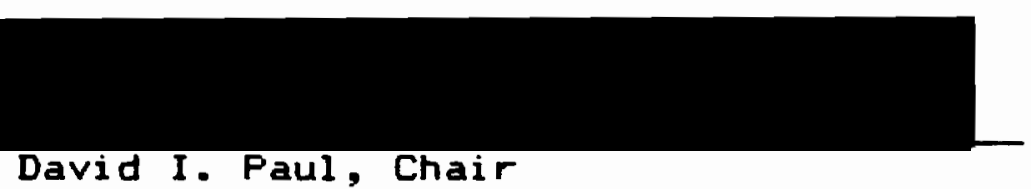

David I. Paul, Chair

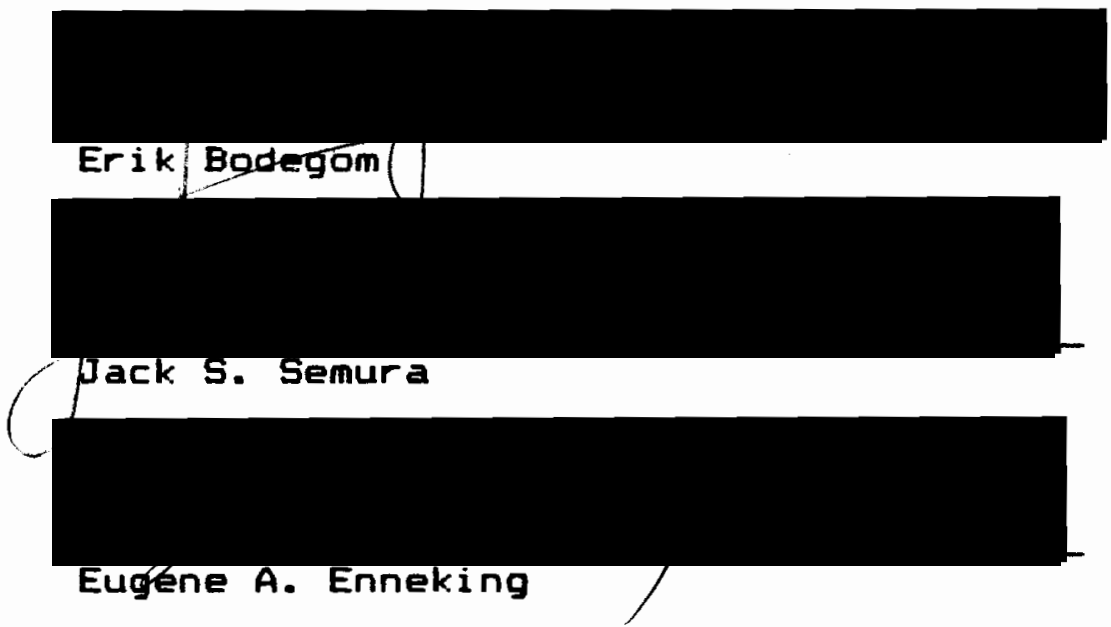

A modified Witten-sander algorithm was devised for the diffusion-limited aggregation process. The simulation and analysis were performed on a personal computer. The fractal dimension was determined by using various forms of a twopoint density correlation function and by the radius of gyration. The results of computing the correlation function with square and circular windows were analyzed. The correlation function was further modified to exclude the 
edge from analysis and those results were compared to the fractal dimensions obtained from the whole aggregate. The fractal dimensions of $1.67 \pm .01$ and $1.75 \pm .08$ agree with the accepted values. Animation of the aggregation process elucidated the limited penetration into the interior and the zone of most active deposition at the exterior of the aggregate. 
A FRACTAL ANALYSIS DF DIFFUSION LIMITED AGGREGATION

BY

CLIFF MYERS

A thesis submitted in partial fulfillment of the requirements for the degree of

\author{
MASTER OF SCIENCE \\ in \\ PHYSICS
}

Portland State University

1988 
TO THE OFFICE OF GRADUATE STUDIES

The members of the Committee approve the thesis of

Cliff Myers presented November 16, 1988.

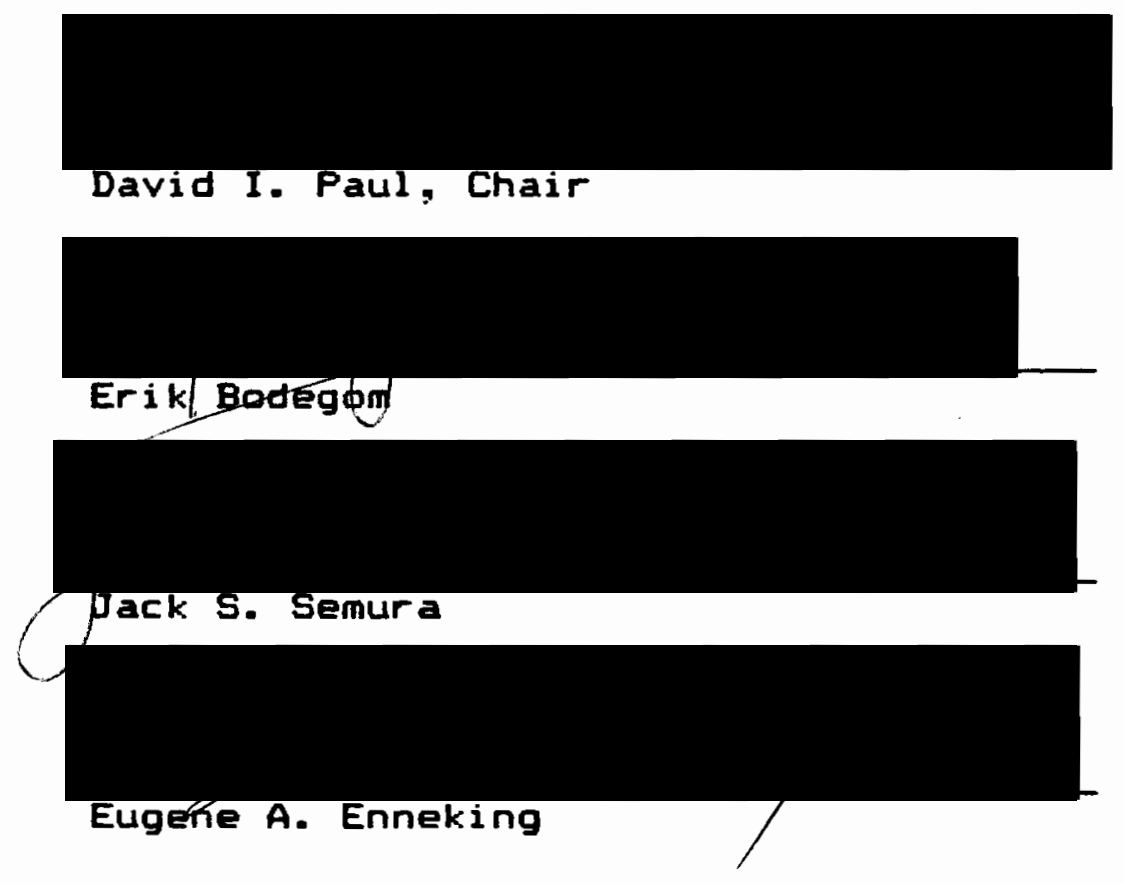

APPROVED:

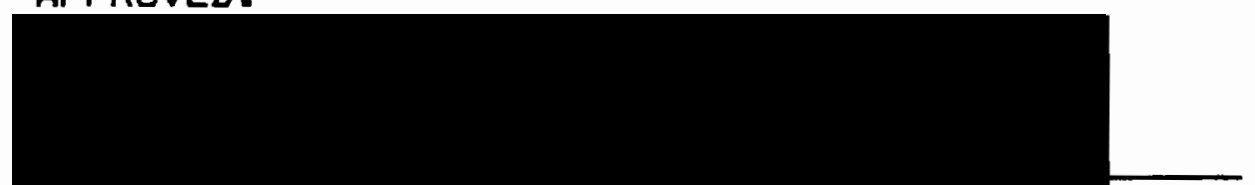

David I. Paul, Chair, Department of Physics

Bernard Ross, Vice Provost for Graduate studies 


\section{ACKNOWLEDGEMENTS}

I would like to thank my friends who stood by me and offered support and encouragement during the time it has taken to complete this "vast project".

I would like to especially extend my gratitude toward Michael E. Sullivan for his expertise and collaboration on the creation of the computer programs and for teaching me that Basic is much more than peeks and pokes, without whom the programs would lack their efficiency and elegance.

To the following members of the the faculty who participated in this project I want to thank:

Dr. Bachhuber for his patient and gentle prodding, to only "kill the flea on the tail of the lion" and for his enthusiasm for computer simulation and encouragement to study fractals.

Dr. Bodegom for his ideas, insights, and effective time devoted to this project.

Dr. Paul for 1 ighting a fire under me.

And to all these people I want to express my appreciation for consistently availing themselves to me.

Finally, I would like to thank B. Jennifer Selliken, who came to my rescue with her proficient editing and helped transform these pages into something I can be proud of. 
TABLE OF CONTENTS

FAGE

ACKNOWLEDGEMENTS $\ldots \ldots \ldots \ldots \ldots \ldots \ldots \ldots \ldots \ldots \ldots \ldots \ldots \ldots$ i $\ldots \ldots \ldots$

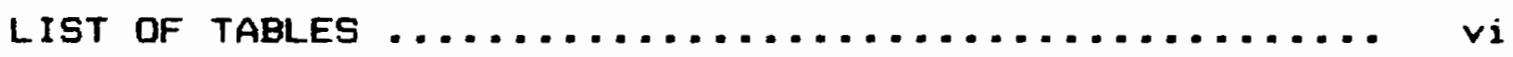

LIST OF FIGURES ........................... vi

CHAFTER

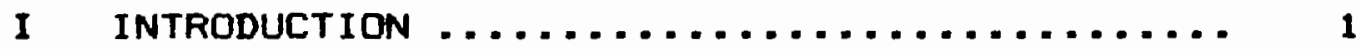

I I BACKGRQUND MATERIAL .................... 4

The Fractal Dimension ................ 4

Density Scaling .................... 5

The Correlation Function ............. 7

The Diffusion-Limited Aggregation Model .. 9

The Laplace Equation ................. 12

Experimental Realizations of the Model ... 14

Electrodeposition

Hydrodynamics

Dielectric Breakdown

I I IMPLEMENTATION OF THE MODEL $\ldots \ldots \ldots \ldots \ldots \ldots \ldots$

IV SIMULATION RESULTS AND DISCUSSION ......... 20

Numerical Results ................... 20

Correlation Function Results

Radius of Gyration Results

Graphical Results ................. 33

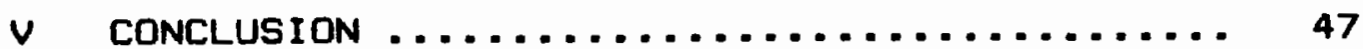


APPENDIX

A THE COMPUTER PRDGRAMS .................. 53

B NUMERICAL DATA ........................ 120

c GRAPHICAL DATA ........................ 137

D ADDITIONAL RADIUS OF GYRATION ANALYSIS ..... 153

E CONSIDERATIONS FDR FURTHER WORK ........... 160 


\section{LIST OF TABLES}

TABLE

PAGE

I Average Fractal Dimensions .............. 22

I Fractal Dimension Data for Individual Small

Aggregates

120

II Fractal Dimension Data for Individual Large

Aggregates

IV Corrected Radius of Gyratiion Results for

Aggregate Number $20 \ldots . . . . . . . . . . . . .156$

$\checkmark$ Corrected Radius of Gyration Results for

Aggregates Numbers 1 to 20, Inclusive... 157 


\section{LIST OF FIGURES}

FIGURE

PAGE

1. Scale Invariance of a Fractal Aggregate ..... 5

2. Sierpinski Gasket ..................... 6

3. Correlation Dependence on 'Window' Size ..... 27

4. Fractal Dimension vs. Aggregate Number ...... 28

5. Radius of Gyration Dependence on Number of

Deposits for Small and Large Aggregates ... 32

6. Aggregate Number $20 \ldots \ldots \ldots \ldots$

7. Quartile Stages of Growth of Aggregate Number

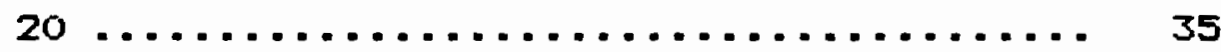

8. Radius of Gyration Dependence on Number of

Deposits for Aggregate Number $20 \ldots 36$

9. Radial Mass Distribution for Aggregate Number

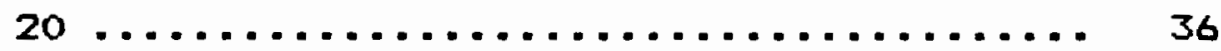

10. Angular Mass Distribution for Aggregate

Number 20 (Smoothed) ................ 37

11. Mass Distribution in $X$ for Aggregate Number

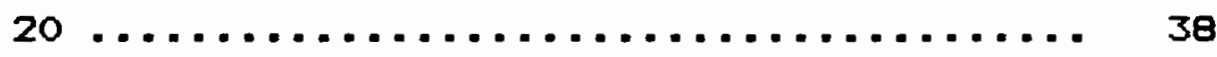

12. Mass Distribution in $Y$ for Aggregate Number

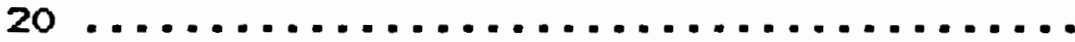

13. Upper Percentiles of Deposition for Aggregate

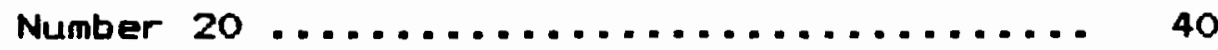


14. Cumulative Probability Distribution in $x$ and

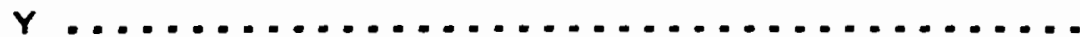

15. Cumulative Angular Mass Distribution of the 30 Large Aggregates (Smoothed)

16. Cumulative Mass Distribution in $x$ for the 30

Large Aggregates .....................

17. Cumulative Mass Distribution in $Y$ for the 30 Large Aggregates

18. Cumulative Radial Mass Distribution for the

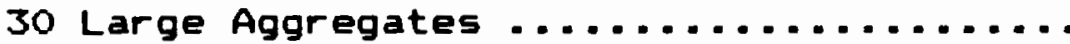
46

19. Persistence of Growth Trends ............. 46

20. Demonstration Program Flowchart .......... 55

21. Ln $\left(R_{0}\right)$ vs. Ln $(N)$ for Aggregate Number $1 \ldots 122$

22. Ln $\left(R_{0}\right)$ vs. Ln(N) for Aggregate Number $2 \ldots 122$

23. $\operatorname{Ln}\left(R_{0}\right)$ vs. Ln (N) for Aggregate Number $3 \ldots 123$

24. Ln $\left(R_{0}\right)$ vs. Ln(N) for Aggregate Number $4 \ldots 123$

25. Ln $\left(F_{0}\right)$ vs. Ln(N) for Aggregate Number $5 \ldots 124$

26. Ln $\left(R_{0}\right)$ vs. Ln (N) for Aggregate Number $6 \ldots 124$

27. Ln (Ro) vs. Ln(N) for Aggregate Number $7 \ldots 125$

28. Ln $\left(R_{0}\right)$ vs. Ln(N) for Aggregate Number $8 \ldots 125$

29. Ln $\left(R_{0}\right)$ vs. Ln(N) far Aggregate Number $9 \ldots \ldots$

30. $L N\left(R_{0}\right)$ vs. Ln(N) for Aggregate Number $10 \ldots 126$

31. Ln(R०) vs. Ln(N) for Aggregate Number $11 \ldots 127$

32. Ln $\left(R_{0}\right)$ vs. Ln(N) for Aggregate Number $12 \ldots 127$

33. Ln $\left(R_{0}\right)$ vs. Ln(N) for Aggregate Number $13 \ldots 128$

34. Ln (Ro) vs. Ln(N) for Aggregate Number $14 \ldots 128$ 
35. Ln $\left(R_{0}\right)$ vs. Ln(N) for Aggregate Number $15 \ldots 129$

36. $\operatorname{Ln}\left(R_{0}\right)$ vs. Ln (N) for Aggregate Number $16 \ldots 129$

37. Ln (Ro) vs. Ln (N) for Aggregate Number $17 \ldots 130$

38 Ln $\left(R_{0}\right)$ vs. Ln(N) for Aggregate Number $18 \ldots 130$

39. Ln $\left(R_{0}\right)$ vs. Ln (N) for Aggregate Number $19 \ldots 131$

40. Ln (Re) vs. Ln(N) for Aggregate Number $20 \ldots 131$

41. $\operatorname{Ln}\left(R_{0}\right)$ vs. Ln(N) for Aggregate Number $21 \ldots 132$

42. $\operatorname{Ln}\left(R_{0}\right)$ vs. Ln (N) for Aggregate Number $22 \ldots 1.32$

43. Ln $\left(R_{0}\right)$ vs. Ln (N) for Aggregate Number $23 \ldots 133$

44. $\operatorname{Ln}\left(R_{0}\right)$ vs. $\operatorname{Ln}(N)$ for Aggregate Number $24 \ldots 133$

45. $\operatorname{Ln}\left(R_{0}\right)$ vs. Ln(N) for Aggregate Number $25 \ldots 134$

46. $\operatorname{Ln}\left(R_{0}\right)$ vs. Ln(N) for Aggregate Number $26 \ldots 134$

47. Ln (Ro) vs. Ln(N) for Aggregate Number $27 \ldots 135$

48. Ln (Ro) vs. Ln(N) for Aggregate Number $28 \ldots 135$

49. Ln (Ro) vs. Ln (N) for Aggregate Number $29 \ldots 136$

50. Ln $\left(R_{0}\right)$ vs. Ln(N) for Aggregate Number $30 \ldots 136$

51. Corrected Radius of Gyration Dependence on

Number of Deposits for Aggregate Number 20. 156

52. Corrected Radius of Gyration Dependence on

Total Number of Deposits for 26 Small and

Large Aggregates ....................... 158 


\section{CHAPTER I}

\section{INTRODUCTION}

Many comple: forms in nature are products of some kind of growth process. There are growth processes ranging from the formation of galaxies to polymers, from the structure of snowflakes to that of living systems. It is hoped that insight into the underlying mechanisms of growth and the formation of structure can be gained from exploration of more tractable models than the direct study of these complicated physical systems. Researchers have been recently encouraged by the intricate patterns and scaling relations that can be produced by computer simulations. By using few and simple growth rules it is suggested that the computer models can elucidate some of the essentials of the mechanisms of growth.

Many everyday forms have the property of selfsimilarity, that is, the appearance of the structure is invariant under change of length scale. Familiar examples include coastlines, rivers, and lightning. The quantitative description of the structure of these forms, which had been until recently regarded as too complicated, has been facilitated by the concept of the fractal dimension, which was primarily developed by Mandelbrot in 1975. It has 
provided the tool for understanding a diverse variety of processes which lead to similar fractal geometries. Aside from scientific considerations, structures with fractal geometries are found in many processes and products of technological importance, such as, aggregates and fluid flows.

The other development which has stimulated much recent research is the Witten-Sander model of diffusion-limited aggregation (1981). The fractal graphical output produced by the computer simulation bears a striking resemblance to actual structures and patterns found in nature, examples of these include: cathodic deposition, dielectric breakdown, and viscous fingering. These physical growth processes and the stochastic growth rules of the simulation can be related to a potential field described by Laplace"s equation. Moreover, computation of the fractal dimension has been verified by direct experimental measurement. This suggests that the model provides a basis for understanding previously unrelated processes and that computer simulation can serve as a bridge between theory and experiment.

I have devised a modified Witten-Sander algorithm for the diffusion-limited aggregation process and performed the simulation and analysis on an Atari 1040ST personal computer. After generating the patterns, the fractal dimension was computed by using a two-point density correlation function and compared to that obtained using the 
radius of gyration. The method of computing the correlation function was modified to study edge effects. Frequency histograms were obtained for various coordinate systems to investigate any defects in the simulation. Animation programs were written to demonstrate the active zone of deposition and to better illustrate the deposition process. After presentation of background material and details of the model, the method of simulation and programming details are then discussed. Following that, the graphical and numerical results are analyzed and compared to similar theoretical and experimental studies. Concluding remarks are then offered in support of the accepted fractal dimension for diffusion-limited aggregation. Additionally, comments are presented to address the differences between the methods for computing the fractal dimension. 
CHAPTEF II

BACKGROUND MATEFIAL

THE FRACTAL DIMENSION

Mandelbrot has extended the application of geometrical constructs to the natural sciences by generalizing the scaling relationships found in certain mathematical functions and geometric patterns. These had been previously disregarded as pathological, to the forms common in nature. He recognized that fractal forms could serve as tools for analyzing physical phenomena. Fractal geometry may become better suited to deal with the real world of intricacies and irregularities than the Euclidean idealizations of abstract regular forms of smooth curves and surfaces.

The concept of fractal dimension, subsequently referred to in this thesis as $D_{\text {, }}$ is demonstrated by considering the diffusion-limited aggregate grown by the simulation in the embedding Euclidean dimension, $d=2$, as having a fractional dimension such that $1 \leq D \leq d$ (Figure 1.). The aggregate is not a compact surface punctured with holes, nor is it a meandering line, it is a fractal (except on the scale of pixels). The irregularities are not without order in that fractals have an intrinsic symmetry, the property of self-similarity, although for random 


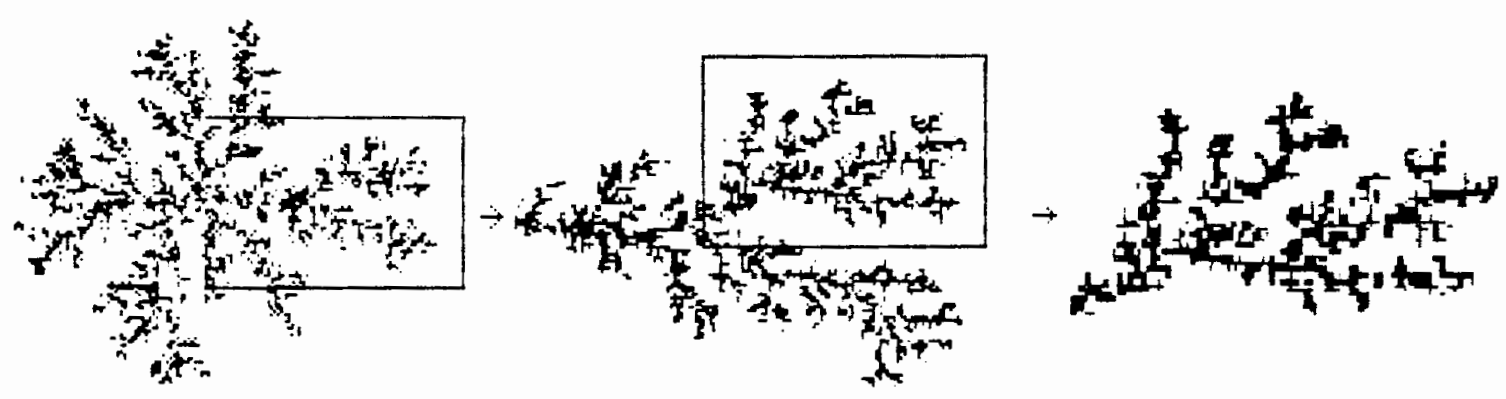

Fiqure 1. Scale invariance of a fractal aggregate.

fractals this dilation symmetry is statistical.

Although the structure is grown by a random process, it is not random. As the sections of the structure are magnified the pattern is recognizable so that similar structure exists on all scales between an upper cut off. nearly the size of the aggregate and a lower cut off, on the order of a pixel diameter. Thus, there exist 'holes' at all length scales. A purely random pattern would not show this scaling of 'holes'. As a consequence of having 'holes' of all sizes, the pixel density decreases with increasing length scale. This can be contrasted with a homogeneous object of Euclidean geometry where the density is independent of the length scale on which it is measured.

\section{DENSITY SCALING}

The fractal dimension is a measure of how density approaches zero as the length over which it is measured increases (assuming that there is no upper cut off). The functional equation, $M(\lambda L)=\lambda d M(L)$ with $\lambda>0$, describes 
how the mass of Euclidean objects scale with length. This is analogous to regular fractal objects such as Sierpinski gaskets. These can also be described by $M(\lambda L)=\lambda$ M $(L)$ with $D<d$ C D is also called the similarity dimension since it describes how the mass changes after a change of scale, $\lambda$. ) (Figure 2.) The solution for the fractal mass dependence on size is obtained by use of $\lambda=L^{-2}$ and $M(1)=1$ and is

$$
M(L)=L D .
$$

The density, $\rho$, given by $\rho=M / L d$ for exact fractals is

$$
\rho=L^{\mathbf{D}-a} \text {. }
$$
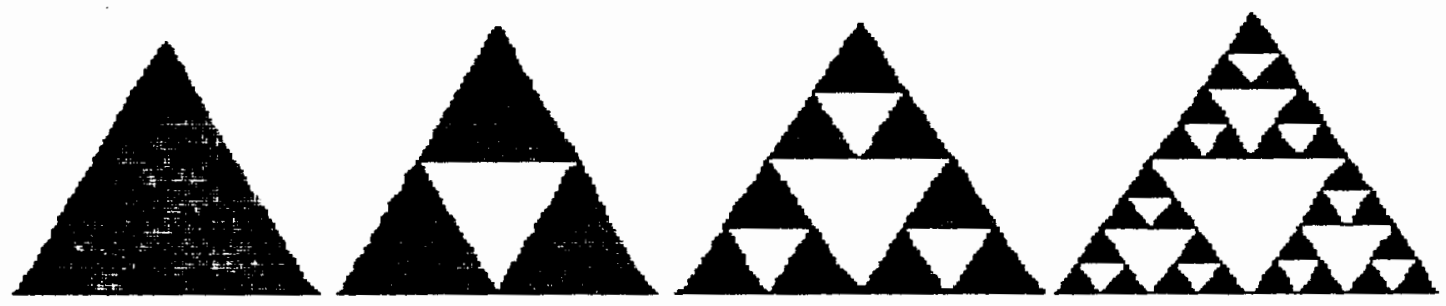

Fiqure 2. Sierpinski gasket.

For the Sierpinski gasket of figure 2, the mass scales according to $M(2 L)=3 M(L)=2^{D M}(L)$ and $D=\ln 3 / 1 n 2 \cong 1.585$. Although, for exact fractals such as Sierpinski gaskets the fractal dimension can be calculated due to their deterministic construction rules; the fractal dimension for diffusion-limited aggregates grown with a stochastic process can only be measured.

The fractal dimension, as introduced, corresponds to the mass dimension in physics and any characteristic length such as the radius of gyration can be used to relate an aggregate"s mass to its size during the process of growth. 
In a general way, the fractal dimension can be defined by:

$$
N(r)=\left(r / r_{0}\right) D
$$

where $N(r)$ is the quantity obtained by measuring a fractal medium with a gauge ro. Forrest and Witten (1979) first obtained for aggregated smoke particles that $M(L)=L^{2}-L^{4}$ and concluded that there were long range correlations in the particle density. There is another, less globally defined formulation for the fractal dimension, it is the correlation function, $C(r)$, which must also reflect the scale invariance.

\section{THE CORRELATION FUNCTION}

The correlation function, $c(r)$, may be defined as the average density of an aggregate at a length $r$ from occupied sites and, as such, it is a local measure of the average environment of a site, $C(r)=N^{-1} \Sigma \delta\left(r_{1}+r\right) \delta\left(r_{1}\right)$ summed over the occupied sites, $r_{1}, i=1, \ldots . N$. The correlatian function thus describes the probability that a site within a length $r$ is occupied. The probability of occupancy is the ratio of occupied sites to the total sites of possible occupancy. Using equation (2), the correlation function is:

$$
C(r)=r^{-\infty} r^{-\infty}=r^{-\infty}=r^{\infty} \text {. }
$$

Witten and Sander (1981) first noticed that the correlation function for diffusion-limited aggregates was consistent with a power $1 \mathrm{aw}$, and found $C(r)=r^{-0.343}$. The correlation function is scale-invariant in that $C(\lambda r)=\lambda^{\infty} C(r)$. 
Although, globally, the density of the aggregate decreases as it grows, (due to the corresponding growth in the "hole" size distribution) locally, these unoccupied sites between the extending tenuous arms do not affect the correlation function if $r \ll<$ Lmax. It is the screening effect of these growing arms that allows for fractal, as opposed to compact growth. That is, it allows for the long range correlations in the pattern, and the decrease in aggregate density.

Aggregation processes can be roughly classified into three regimes. The first of these is when an object grown near equilibrium, such as a crystal, which has only short range correlations. This correlation length or resemblance distance is on the order of the unit cells of the crystal. When the system is driven away from equilibrium, growth is in the second regime. For example, in supercooled solidification, the morphology becomes that of dendritic pattern formation where the structure may still be regarded as compact. The lengths associated with the steady-state growth of the intricate patterns of snowflakes are much longer than the crystalline lattice spacing (see Langer, 1980). The third regime, applies to diffusion-limited aggregation in which the growth process is irreversible and its growth is even farther from equilibrium. It has long range density correlations and no natural length scales, evident by its having holes of all sizes. 
THE DIFFUSION-LIMITED AGGREGATION MODEL

In the Witten-Sander model for diffusion-limited aggregation or DLA, pixels are added one at a time to the growing aggregate, via random walk trajectories on a lattice. The process is started with a single seed at the lattice origin. Subsequent pixels are introduced from random points sufficiently distant so that their flux is isotropic. They then undergo simulated Brownian motion until a site adjacent to the aggregate is reached, where they irreversibly 'stick" without rearrangement.

Various improvements and extensions to this process have been developed, beginning with the work of Meakin (1983a). Meakin injected the random walkers from a random point on a circle of radius five lattice spacings greater than the distance from the seed to the most distant pixel on the growing aggregate, $R_{x N o E c t}=R_{\max }+5$. The random walker was also 'killed" if $R>R_{k x L L}=3 R_{\max \text {. }}$

With an average aggregate size of 9700 pixels, Meakin obtained fractal dimensions, of $1.68 \pm .04$ and $1.68 \pm .07$ taken from calculations using the radius of gyration and a correlation function, respectively.

In order to investigate lattice effects, the sticking rules were modified. The particle was incorporated into the aggregate if it reached a next-nearest neighbor position and did not stick if it was at the nearest neighbor position. 
The corresponding dimensions of, $1.69 \pm .07$ and $1.70 \pm .07$ were obtained for aggregates with an average size of 5900 pixels.

In order to investigate the effects of the "sticking" probability on the fractal dimension, the probability was set at 0.25 for nearest neighbor sites and 0.0 for the nextnearest neighbors. The aggregates, with an average size of 16.300 pixels, yielded fractal dimensions of, $1.71 \pm .055$ and $1.73 \pm .13$ respectively. Setting the probabilities at 0.0 for nearest neighbor sites and 0.1 for the next-nearest neighbors, Meakin further obtained the fractal dimensions of, $1.74 \pm .03$ and $1.73 \pm .04$ respectively, for aggregates with an average size of 9,800 pixels.

Later improvements in the simulation algorithm include those by Meakin (1983b) where the aggregation rate was increased by scaling the step size of the random walk to the distance from the aggregate. The step size was increased to two lattice units if the random walker was at a distance greater than rmax + 5 lattice units from the center seed, four units, if greater than $r_{\max }+10$ units, four, if greater than $r_{\text {max }}+20$, eight if greater than $r_{\text {max }}+40$, and sixteen if $r_{\max }+80$. The correlation function was calculated for $5 \leq r \leq 50$ and gave a fractal dimension of, $1.68 \pm .05$. The radius of gyration gave a fractal dimension of. $1.73 \pm .06$. These results were obtained from aggregates whose average size was 8,585 pixels. 
It can be seen that, for these relatively small aggregate sizes (Meakin states that these aggregate sizes reached the practical 1 imit for the VAX-11/780 computer which was used), the fractal dimension obtained by radius of gyration calculations agreed well with those that were based on the correlation function. Furthermare, the results were not significantly changed by the described modifications in the simulation process.

The diffusion-limited aggregation model was developed to provide a simple model for a broad class of growth processes in which diffusion limits the rate of irreversible growth. The reason that the model produces fractal growths and not non-symmetric amorphous blobs can be qualitatively explained by the interplay of noise and growth. Consider the random deposition of a few nearby particles; tiny bumps and "holes' will be formed due to noise of the Brownian process. The bumps will grow faster than the interior of the "holes" because the probability that the random walking particles will arrive at the bumps, is greater. (This is demonstrated by the lightning rod effect in electrostatics.) As the bumps become steeper, the deposition probability decreases for the interior of the "holes": The bumps grow larger due to this screening effect and tiny bumps, in turn, begin to form on them, then subsequent splitting occurs and this gives rise to the ramified fractal structure. This evident growth instability is similar to the Mullins-5ekerka 
instability of solidification processes. The association between diffusion-limited aggregation and certain processes of electrostatics electrolytic deposition and dielectric breakdown), thermal-mass transport (dendritic solidification), and hydrodynamics (viscous fingering) is more than similar growth instabilities, or structure. Although these processes apparently do not involve diffusing "particles', the 'particles' are conserved and under appropriate conditions they can all be described by harmonic functions which satisfy Laplace"s equation.

\section{THE LAFLACE EQUATION}

That the random walkers diffuse can be understood by noting that the probability that the $x$ site is reached on the $k+1$ step is: (following Witten and Sander, 1983)

$$
u(\underline{x}, k+1)=1 / 4 \Sigma u(\underline{x}+\underline{1}, k) \text {, }
$$

where the summation over $\underline{1}$ runs over the 4 neighbors of $\underline{x}$ and is simply the previous mean value of the neighboring sites. Without boundaries to distort the probability field, the randon walk will eventually diffuse everywhere (In the simulations, it is hoped that the random walker has no preferred direction.) In the continuum limit, this becomes the diffusion equation for the probability distribution of an incoming particle cequivalent to the average concentration if many were simultaneously diffusing), with $B$ as the diffusion constant: 


$$
a / a t=B \nabla u
$$

The boundary conditions for DLA are given by the simulation rules: because the particles deposit on the growing aggregate $u=0$ on the perimeter and because the particles approach isotropically $u=u_{-}$for $\underline{x} \rightarrow \infty$. Because only one walker arrives at a time, they' see', essentially a steady-state; that is, each deposit's perturbation of the field relaxes instantaneously. Thus, the diffusion equation reduces to Laplace's equation, outside the aggregate:

$$
\nabla_{u}=0
$$

More formally, the probability distribution is analogous to a potential field, the gradient of which, is proportional to the diffusion flux of random walkers. Because the walkers are absorbed only on the perimeter, the flux, $\underline{v}$, has zero divergence $\left(\underline{v} \sigma \nabla, \nabla^{*} \underline{v}=\nabla_{u} u=0\right)$. The growth of the aggregate is given by the flux at its surface. The varied physical systems of: solidification, electrodeposition, fluid-fluid displacement, and aggregation, under appropriate approximations, all share similar interfacial growth equations and morphologies. The corresponding control variables for these systems are; undercooling, applied voltage, pressure, and concentration. For example, in electrodeposition, the potential is the electric potential, $V$, where the growth rate is proportional to the electric field, E, at the surface of the deposit (E $\mathbb{E}-\nabla, \nabla^{*} E=0$, and $\left.\nabla^{*} V=0\right)$. 
EXPERIMENTAL REALIZATIONS OF THE MODEL

\section{Electrodeposition}

Using a polymer to raise the viscosity of the copper sulfate electrolyte so as to inhibit the mixing of the sulfate ions by convection, and an added excess of sodium sulphate to screen the electric field, Brady and Ball (1984) deposited copper in which growth was limited by diffusion of Cuz+ ions. The radius of deposit was proportional to the diffusion-limited current and the mass was obtained from Faraday's law. The inferred fractal dimension obtained was $2.43 \pm .03$ which is in agreement with three dimensional simulations of DLA.

Two dimensional zinc leaves were grown by Matsushita et al. (1984) and their two-point correlation function was obtained by digitized image analysis. The deposits grew in an interfacial layer between a zinc sulphate solution and a covering of n-butyl acetate. Because the applied voltage was low, the growth process was controlled by the electrical potential field, obeying Laplace's equation. The fractal dimension obtained was $1.66 \pm .03$.

\section{Hydrodynamics}

Hele-Shaw cells consisting of two parallel plates where a low viscosity fluid, is injected into a high viscosity fluid have been used as analogs for fluid flow through homogeneous porous media. By Darcy's law, the local 
fluid velocity is proportional to the pressure gradient, and for an incompressible fluid, the fluid potential field obeys Laplace's equation. Paterson (1984) was the first to point out the similarities between the viscous fingers produced by the Saffman-Taylor instabilities and the patterns of DLA. He speculated that they should also scale like DLA.

Daccord et al. (1986) used water as the driving fluid and a high viscosity polymer for displaced fluid. The boundary conditions agreed with those of DLA because the viscosity of the water was negligible which allowed the approximation that the interface be isobaric. However, the polymer was non-Newtonian and its shear thinning introduced a non-linearity which was accounted for by using a power function of the pressure gradient. The fractal dimension was measured using various methods which produced consistent results of, $1.70 \pm .05$.

\section{Dielectric Breal:down}

Lichtenberg figures are the electrical discharge patterns formed by the conduction channels during dielectric breakdown. Niemeyer (1984) assumed that the breakdown channel is a good enough conductor to be regarded as an equipotential and that further breakdown or growth of the breakdown channel is proportional to the surrounding electric field (or the gradient of the electric potential). Under these crude approximations the electric potential obeys Laplace's equation with similar boundary conditions as 
DLA. In compressed SFo gas, the surface discharge on a plate of glass was analyzed and a fractal dimension of 1.7 was found from digitized photographs. 


\section{CHAPTEF III}

IMPLEMENTATION OF THE MODEL

Various modifications to Meakin's improvements on the original Witten-Sander model were made due to machine Iimitations and the desire to have real-time graphics display. (For more extensive discussion of these modifications see the Appendix A.) The most notable of these is the modification of the interfacial boundary conditions. In consideration of memory and speed Iimitations, the growth interface or exterior perimeter was not stored separately from the aggregate as it was grown. Consequently, the deposition rules at the interface were changed so that the pixel was deposited only when it attempted to "jump" into the aggregate and not when it was on its interface. Thus interfacial transport was allowed and the deposition probability as a function of the velocity relative to the interface, $P(v)$, was as follows:

$$
\begin{gathered}
P\left(-V_{\text {NORMAL }}\right)=1 \\
P\left(+V_{\text {NOMMAL }}\right)=P\left( \pm V_{\text {TANGENTIAL }}\right)=0 .
\end{gathered}
$$

Deposition occurred at the site from where it attempted to "jump" into the aggregate. As the pixel was only allowed to single step while inside the deposition zone, $R \leq F_{\max }+5$, and because the steps were along the orthogonal lattice 
directions, the possibility of the pixel "jumping" over a deposit filament was eliminated.

In Meakin's model the deposition forces acted over a distance of one pixel diameter, since deposition occurred as soon as the pixel entered the one pixel thick perimeter. This is in contrast to the contact forces of the model used in this study, which allowed the pixel to move tangentially along the interface until an attempted' jump' caused the centers of the pixels to coincide. In this sense, the present study deals with aggregation of points and ignores the excluded volume effect, whereas Meatin's model aggregated extended pixels of one lattice spacing in diameter. Consequently, the surface variations on the order of a lattice spacing were not smoothed over, which was an effect of the overlapping of the surrounding perimeter layer in Meakin's model. Thus, pixels could enter into cavities with entrances of one pixel in diameter and there be deposited. However, this modification did not significantly change the fractal dimension, which is a measure of the local deposit density or compactness.

The growing aggregation was surrounded by a 'birthing" circle which injected the random-walking pixels at a distance of $F_{\text {Invect }}=R_{\text {max }}+5$ lattice spacings away from the initial center seed. The release was randomized over half-degree increments around this circle. If the pixel was outside of this circle the step size was scaled as follows: 
if $10^{*} 2^{N}<R-R_{\max }<10^{*} 2^{N+2}$ then stepsize $=2^{N+2}$. The random walk was continued until deposition occurred or until the pixel was terminated on the 'killing' circle of radius $R_{x I L L}=2 \cdot R_{\max }+5$. This modification was made to expedite the deposition process.

To complete the description of the model, it should be noted that, although, there were toriodial boundaries (remnants from a previous demonstration program, from which the simulation program evolved), they were never reached because the growth terminated when the aggregate reached a radius of 200 lattice spacings. This constraint was devised to insure that the whole aggregate could be displayed. The center seed was located at $(200,200)$ in the screen space. The coordinates of the seed in the simulation space la Boolean array in main memory) were $(408,408)$ with boundaries at 3 and 812 in both $x$ and $y$. Although, larger aggregates could have been grown, their growth times would have been excessive and it would have been necessary to partition their displays. (For a more complete discussion of the memory and time constraints, see Appendix A.)

Initially, 26 small aggregates were grown using the demonstration program which stopped growth when the 'birthing' circle reached the edge of the screen at $R=200$ lattice spacings. These small aggregates were then used as 'seeds" in the simulation program which allowed for larger growth. A total of 30 large aggregates were grown. 
CHAPTER: IV

SIMULATION RESULTS AND DISCUSSION

NUMERICAL RESULTS

The output from the simulation program consisted of two files which were stored on disk. The spatial deposit array was stored as a sequential file in the order of deposition. The screen buffer was also stored as a binary file so that screen sites could be later checked for deposition. These files were processed by programs to obtain the fractal dimension from the correlation function and the radius of gyration. (For more extensive discussion of these programs see Appendix A.)

The correlation program actually consisted of three separate programs, each of which calculated the correlation function using circular and square 'windows', and from its dependence on the "window" size, the fractal dimension was determined for each aggregate. The first of these programs used circular "windows" which accumulated the enclosed pixel area by a polygonal approximation which in effect included the pixel area as either inside or outside the "window". This approximation technique affected only those pixels which were on the perimeter of the "window'. This correlation function was evaluated at all the deposits 
comprising the aggregate. The second and third programs excluded those pixels located at radii, $\dot{R}>R_{\max }=32.5$ lattice spacings as, 32.5 was the 1 argest window size. Because the edge of the growth was where deposition was most active, it was thought that by excluding the edge from consideration, the fractal dimension obtained would be more representative of the complete aggregate. The third correlation program utilized a look-up table of the exact areas for those pixels that were bisected by the perimeter of the circular "window'. The "window' sizes for all the programs were $2^{N}+.5$ lattice spacings, $N=0,1,2,3,4,5$. All the correlation programs were tested for accuracy by evaluation of the fractal dimension of compact Euclidean figures.

The radius of gyration program used the lattice origin and not the center of mass of each aggregate to compute the radius of gyration. The calculation of the center of mass at each deposition would have greatly increased the process time. Furthermore, it was assumed that any offset would not be appreciable. If it was appreciable, it would distort the numerical results in a complicated manner.

\section{Correlation Function Results}

For each aggregate, the results of the dependencies of $L n(C(r))$ on $L n(r)$, and $L n\left(R_{0}\right)$ on $L n(N)$ were analyzed by linear regression to give the corresponding fractal dimensions. The individual results are given in Appendix $B$. 
Each of the 26 small aggregates served as a seed for the growth of the large aggregates. The correlation results of all the individual aggregates were averaged by a separate least squares analysis of the average results of each 'window'. The average fractal dimension, as determined from the radius of gyration, was determined by processing a composite of all individual growths. (This composite was also utilized in the determination of the frequency histograms, which are discussed below under Graphical Results.) These results are listed in the following table.

\section{TABLE I}

AVEFIAGE FRACTAL DIMENSIONS

Fracta: Diaens:on froe Average Correlation 'Hindow' Data

Souares $\frac{\text { Inclutina Edoe }}{\text { 'Circles' }} \quad$ Souares $\quad \frac{\text { Excludino Edqe }}{\text { 'Circles' }}$ Circles

Said Aqureqates

$\begin{array}{llllll}\underline{D} & 1.66410592 & 1.610013451 & 1.6953093637 & 1.6393097109 & 1.6952591969 \\ \underline{5 . d_{1}} & .0082032213497 & .0079478124734 & .012463779431 & .011922381434 & .012101511144\end{array}$

Large Aggregates

$\begin{array}{llllll}\underline{D} & 1.66684462208 & 1.6107480877 & 1.6725249781 & 1.6160897292 & 1.672937113 \\ \underline{\text { s.d. }} & .005354910725 ? & .0050211512165 & .0058509194604 & .0056517456068 & .0057074275171\end{array}$

Fractal Diension tros Conposite of all Agareqates based on Radius of Grration

Snal! Agqreqates $\quad 1.8452894007 \quad$ Larqe Aggreqates $\quad 1.8120055785$

Average Agoregate Size

Seall Agoreqates $N=4510 \pm 702$ oixels Large Agqreqates $N=16290 \pm 2159$ pixels 
Polygonal approximation of the circular 'windows' was utilized to expedite implementation. Circular 'windows' which computed the exact areas were justified in so far as the correlation function utilized the Euclidean metric. Furthermore, in a statistical sense, the aggregates tended to have a circular symmetry. It had been for computational convenience that Forrest and Witten used square 'windows' to determine the correlations of smoke particles. However, the underlying square lattice geometry also suggests the utilization of the more natural square "windows". In the absence of an adequate discussion of this issue in the literature, it will now be discussed as to whether these computational schemes yielded significant differences of the resulting fractal dimension.

The average fractal dimensions which were obtained by using the correlation function with circular "windows" and by excluding the edges of the aggregates, were, as follows: for the small aggregates, polygonal approximation gave results of D.c.= $1.639 \pm .012$ and exact calculation yielded results of $D_{c}=1.696 \pm .012$. For the large aggregates, results were, $D_{-c}=1.616 \pm .006$ and $D_{c}=1.673 \pm .006$, respectively. Therefore, the polygonal approximation is not justified.

Comparison of the results obtained from the correlation function by using exact circular and square 'windows' and by excluding the edges of the aggregates, 
indicates that the choice of method is arbitrary. Specifically, the fractal dimensions which were obtained far the small aggregates were, for circular and square 'windows"; $D_{c}=1.696 \pm .012$ and $D_{e}=1.695 \pm .012$, respectively, and for the large aggregates the dimensions were identical, $D_{C}=D_{e}=1.673 \pm .006$. Whether structural symmetry or the underlying lattice geometry alter the fractal dimension, as determined by this correlation function, can not be decisively concluded on the basis of this analysis. other correlation functions and scaling relations could be formulated to address this issue more conclusi vely.

The effect of screening on deposition is evident by the decrease of the average fractal dimensions, computed where edges are excluded, as the aggregates become larger. Comparison of the corresponding average fractal dimensions between the small and large aggregates must take into account that the individual large aggregates were grown from individual small aggregate seeds and not independently, each with a particular fractal dimension and growth trend based on its structure. However, because the analysis is based upon the average fractal dimensions, (which suppress any particular trend that an individual aggregate may have in terms of its fractal dimension), it is valid for comparing the change in the fractal dimension between the average small aggregate and the average large aggregate. Because 
the excluded edge is 32.5 lattice spacings for both the small and the large aggregates, the proportion of the region of active deposition that is excluded, is greater for the small aggregates than for the large aggregates. Conversely, proportionately more of the inactive interior region (which is more compact and thus has a greater fractal dimension) is used in the correlation calculation that excludes the edge for the small aggregates rather than for the large aggregates. (Screening, and the active deposition zone, are more fully discussed in the Graphical Results section.)

The average fractal dimensions computed by not excluding the edges of the aggregates and by using the correlation function using square "windows" are; for the small aggregates, $D_{0}=1.664 \pm .008$, and for the large aggregates, $D_{0}=1.667 \pm .005$. The difference in these fractal dimensions is not significant, and is not inconsistent with the above analysis. Furthermore, it suggests that the active zone also scales as a fractal.

The sequence, of the average fractal dimensions, obtained by using the various correlation function schemes, (presented in Table I), is consistent between the small and large aggregates. This is illustrated in Figure 3, on both the graphs for the small and large aggregates, where the slopes of the regression lines are listed in decreasing order. The regression line, for the rejected scheme using polygonal approximation, is skew to those regression lines 
for the exact schemes. The coincident regression lines for the exact schemes: where the edge is excluded, are parallel to the regression 1 ine for the scheme using exact squares, where the edge is included; is true for large aggregates and not for the small aggregates. The regression lines have different intercepts simply because edge deposits were excluded. The average fractal dimensions, calculated by the exact schemes, for the large aggregates, yield the fractal dimension of $D=1.67 \pm .01$. However, the corresponding results, for the small aggregates, do not agree within statistical uncertainty. Further analysis of the average dimensions, between the small and large aggregates, of all the exact schemes, indicates a convergence, as the aggregates become larger, toward the results given by the scheme using squares, and where the calculations included the edge. This convergence is also supported by the agreement between the average fractal dimensions of the small and large aggregates, which are produced by the scheme where the edge is included and the correlation function utilizes squares. This agreement also yields $\mathrm{D}=1.67 \pm .01$. This suggests that, to fully characterize a growing aggregate, an additional fractal dimension for the zone of active deposition could be utilized.

The sequence of the fractal dimensions, obtained by the various correlations schemes, is further illustrated in 
Figure 4. The graphs of the results for the individual small and large aggregates do not intersect, indicating that the consistency of the schemes is not dependent upon the averaging process.
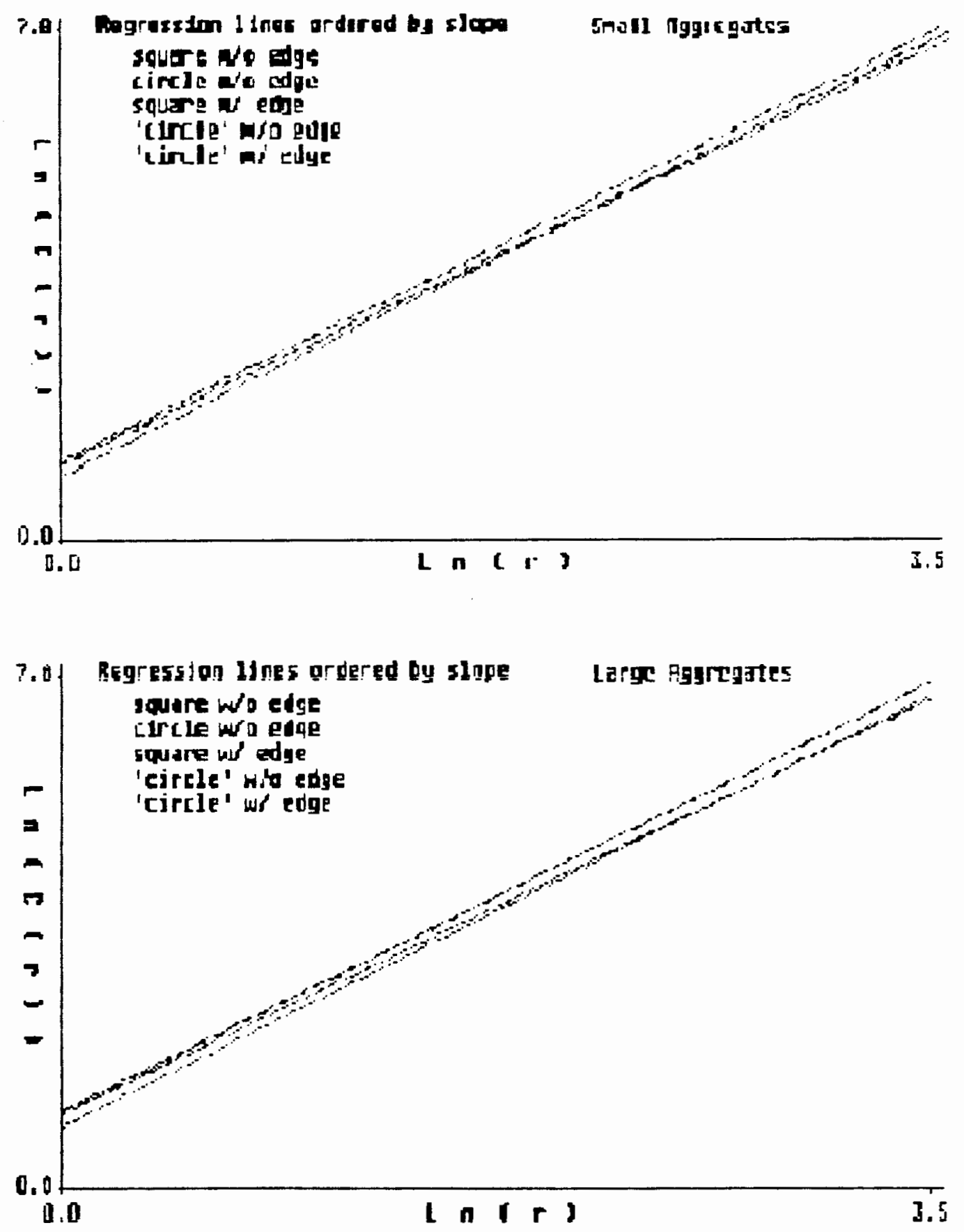

Fiqure 3. Correlation dependence on 'window' size. 

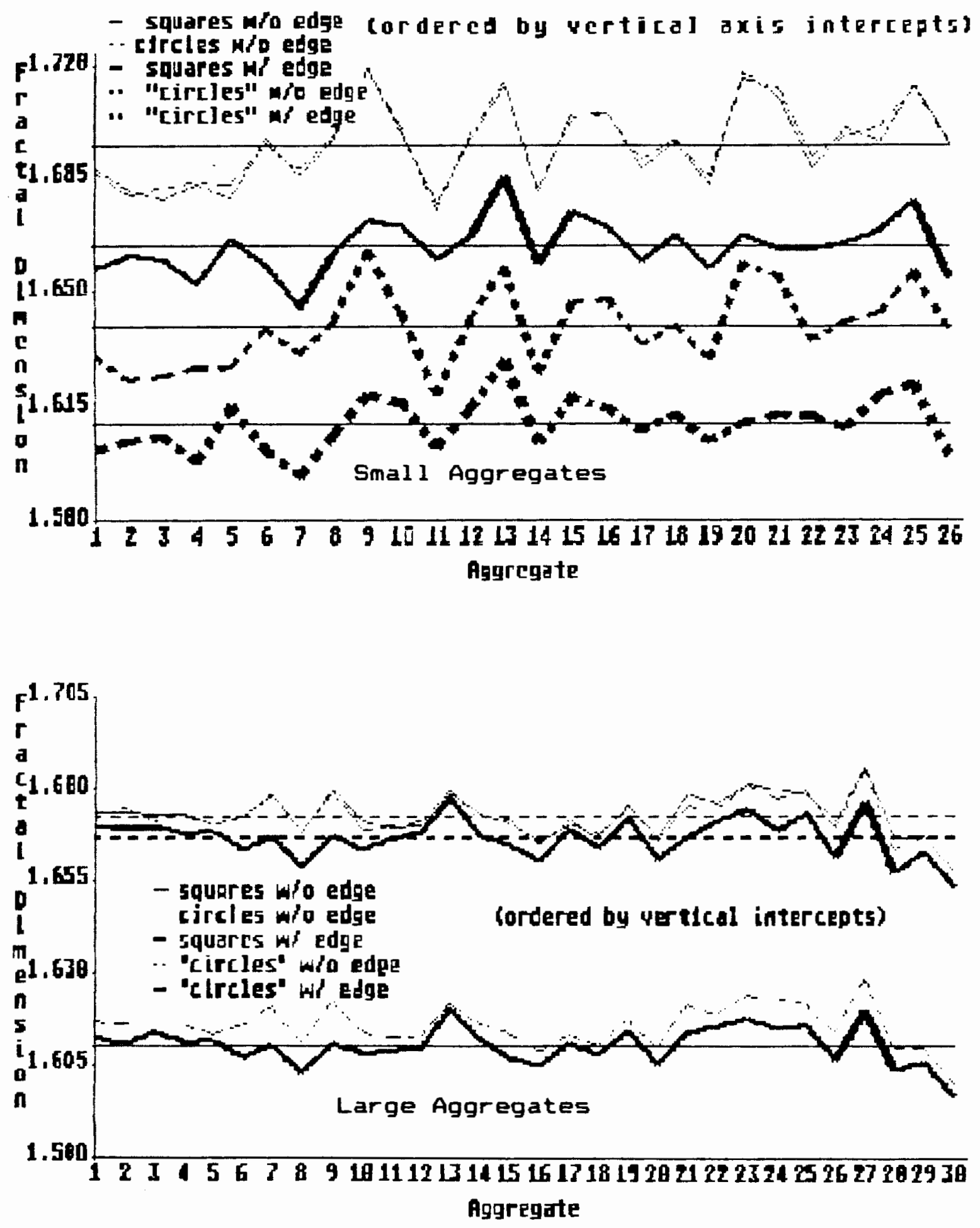

Fiqure 4. Fractal dimension vs. aggregate number. 


\section{Radius of Gyration Results}

The results from the radius of gyration, $R_{0}$, dependence on the number of deposits, $N$, reported in Table I, are not in immediate agreement with the results discussed above concerning the correlation function, $C(r)$, dependence on the "window" size. In further contrast, are the fractal dimensions reported by Meakin, which do agree. (These were similarly related to the slopes of the graphs of Ln(Ro) vs. $\operatorname{Ln}(N)$ and $\operatorname{Ln}(C(r))$ vs. $\operatorname{Ln}(r)$.$) The fractal dimensions,$ calculated from the reciprocals of slopes of the graphs of Ln $\left(R_{0}\right)$ vs. Ln $(N)$, were determined from composites of all the small and large aggregates, over the entire ranges of $N$. Time did not allow for an estimation of the statistical uncertainties associated with the listed fractal dimensions, even though this would have required only minor modifications to the least squares routine in order to obtain the standard deviation of the regression coefficient. However, inspection of any of the $\operatorname{Ln}\left(R_{\bullet}\right)$ vs. $L n(N)$ graphs in Appendix B, indicates that the graphs for the individual aggregates are not initially linear and only appear to asymptotically become so with increasing N. However, due to the condensed size of the graphs, this interpretation may not be valid. The non linear region of the graphs, for small values of $N$, indicates that the aggregates are initially random, and that their structure stabilizes and becomes fractal with more deposition. This corresponds to 
the apparent linear portions of the graph. As an aggregate becomes larger, a deposit's perturbation of the global geometry is diminished. With the average large aggregate size of only $N=16298$, it is unknown whether the fractal dimension also has an upper cut off, above which the aggregate becomes non-fractal, or its dimension approaches another value. It was hoped that the averaging of the individual aggregates into a composite would damp the initial transients and the graph would be linear over its entire range. Indeed, at a first glance, the graphs in Figure 5, appeared to indicate this result. However, when the regression was parameterized by a lower cut off, the resulting fractal dimensions did not stabilize, in fact, the results, as shown in the chart overlaid on the graphs, indicate that the graphs are actually slightly concave. This is in accord with the effect of screening by the perimeter. As the aggregate grows the perimeter effectively leaves behind it a region "frozen" at an intermediate fractal dimension. Deposition, when penetration is restricted, tends to increase the radius of gyration more because it occurs, on the average, at a greater distance. A more thorough study of this concavity and asymptotic growth would require an analysis of the scaling properties of the zone of active deposition. The results which suggest the concavity may lack statistical significance, as the maximum graphical error for the graph of the large 
aggregates is only $\cong 2 \%$. Furthermore, the curve tends to oscillate, which indicates that the graph can be regarded as linear. The use of the upper endpoint, with the parameterized lower cut offs in the linear regression, may not accurately determine the fractal dimension for the average mid region of the aggregates because it tends to attach more statistical weight to the active zone. A separate correlation function analysis of the active zone would determine whether the active zone had a smaller or greater local density than the mid region of the aggregate. Even without this separate analysis, it may be inferred that the active zone had a smaller local density than the mid region of the aggregate. This inference is drawn from an analysis of the results of correlations over the entire aggregate, between those which exclude and those which include, the edge. (These results are listed in Table I.) The question arises, of whether the reported results should represent just the global properties of a stabilized and relatively large aggregate, or whether they should also include the residual effects of its incipient growth. Utilizing the results for an average "mature', yet growing aggregate, the fractal dimensions are, for small aggregates, $D=1.799$, and for large aggregates, $D=1.773$. In acknowledgement of the uncertainties involved, and of the apparent inverse nature of the growth of the aggregate and its fractal dimension, the final result, using the radius of 
gyration is, $D=1.78 \pm .01$. This does not agree with the correlation function results. The relative discrepancy is $\cong 6.6 \%$ The radius of gyration program could be flawed, as there is no obvious explanation for the discrepancy between the two methods (The averages of the individual aggregates,
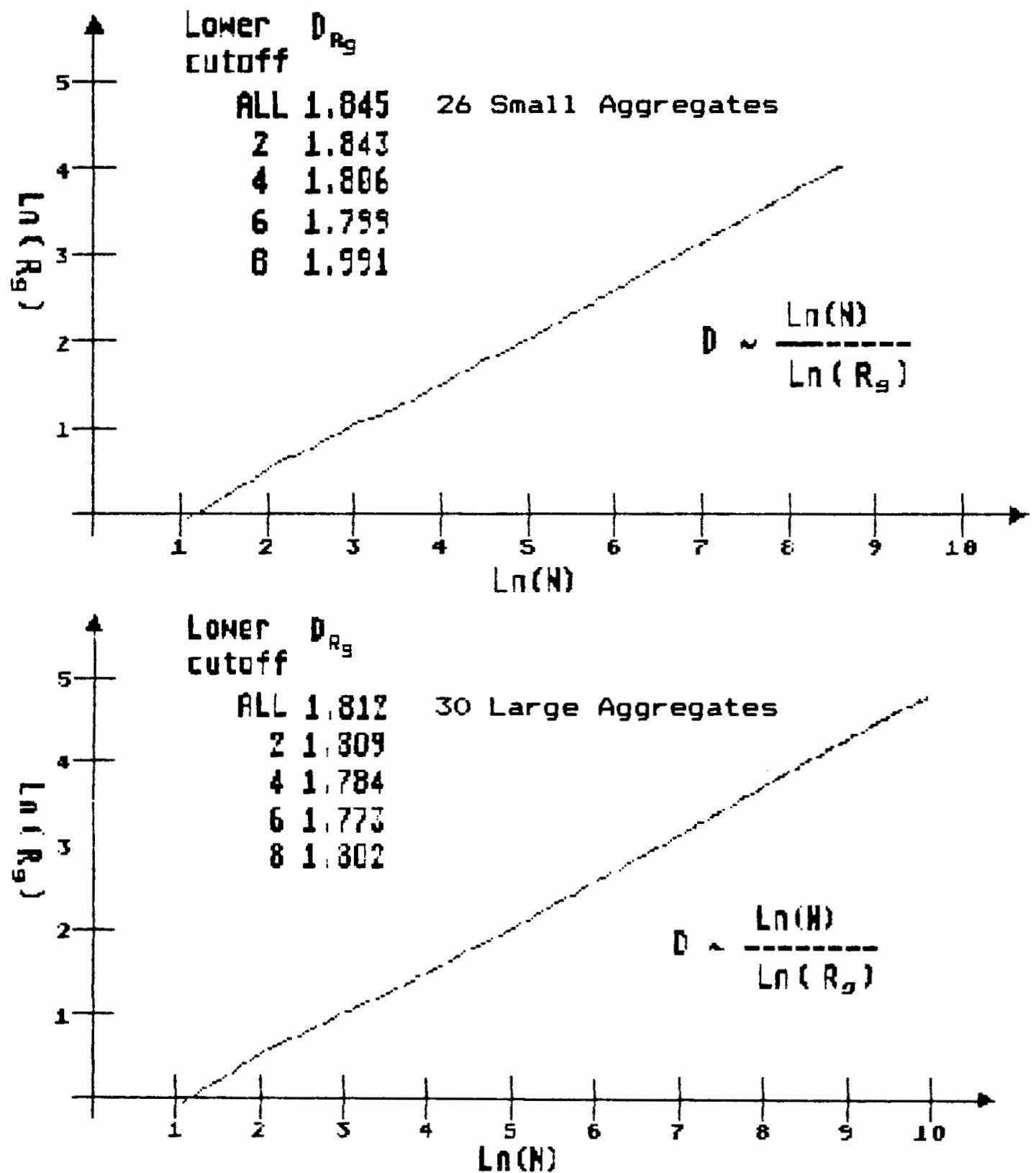

Fiqure 5. Radius of gyration dependence on number of deposits for small and large aggregates. 
without cut off, are, for small aggregates, $D=1.84 \pm .07$, and for large aggregates, $D=1.80 \pm .05$.

GRAPHICAL RESULTS

This section discusses the graphical depiction of the aggregates. The graphical output for all the aggregates are found in Appendix C. It is evident that the aggregates represent a diversity of structure, yet a recognizable pattern is discernable. However, without the fractal dimension, only a qualitative description of this pattern is possible. However, aside from the pattern, other characteristics can be demonstrated. Symmetries and anisotropies were investigated by the use of frequency histograms. The dynamics of growth were studied by use of animation programs, the results of which were distilled into the series of images depicting the evolution of growth. Additionally, the animation programs were used to construct a sequence displaying the depth of penetration at varying stages of screened growth. Aggregate number 20 was selected as a representative aggregate and its characteristics are presented (Figure 6.). A similar presentation follows for the composite of all the large aggregates. The extent that subsequent growth depends upon initial conditions and the persistence of growth trends are studied by the comparison between two of the large growths, which were grown from the same small growth. 
The most salient features are the radial symmetry and the similarity of branching structure ramified over different orders of magnitude. Predicting its occurrence and structure in terms of natural ratios of characteristic lengths, such as arm diameters and interarm distances, unfortunately, was not relevant to the present study, although it certainly merits further study.

Examination of the growth stages of aggregate number 20, in Figure 7, indicates that the initial pattern of the
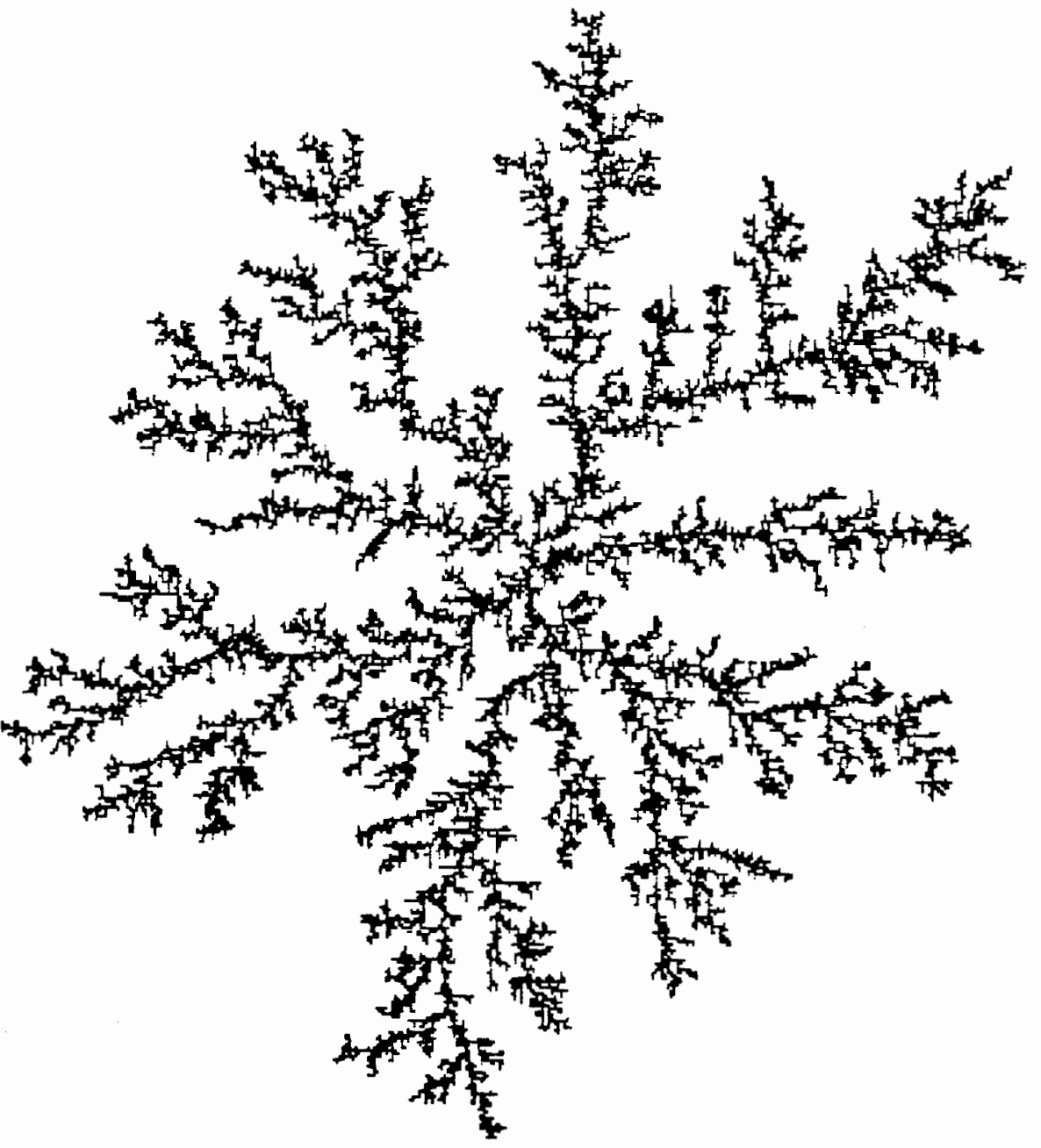

Fiqure 6. Aggregate number 20. 
main branches is propagated, and persists in, the more intricate stages of later growth. The Ln $\left(F_{0}\right)$ vs. $L n(N)$ graph for this aggregate is presented in Figure 8 . The transients of the initial growth are visible in the oscillations of the lower portion of the graph. The frequency histogram of the radial mass distribution is presented in Figure 9. The presence of "holes" is indicated by the increasing portion of the histogram. Growth was terminated before uniformity in the distribution for the mid region of the aggregate could be ascertained.

The radial symmetry is manifest in the outward growth of the arms. The angular distribution, as shown in its frequency histogram in Figure 10, indicates that the arms "sweep up" the incident flux of random walkers. The flux is assumed to be uniform and isotropic. (The unsmoothed data for aggregate number 20 is given Appendix C.)

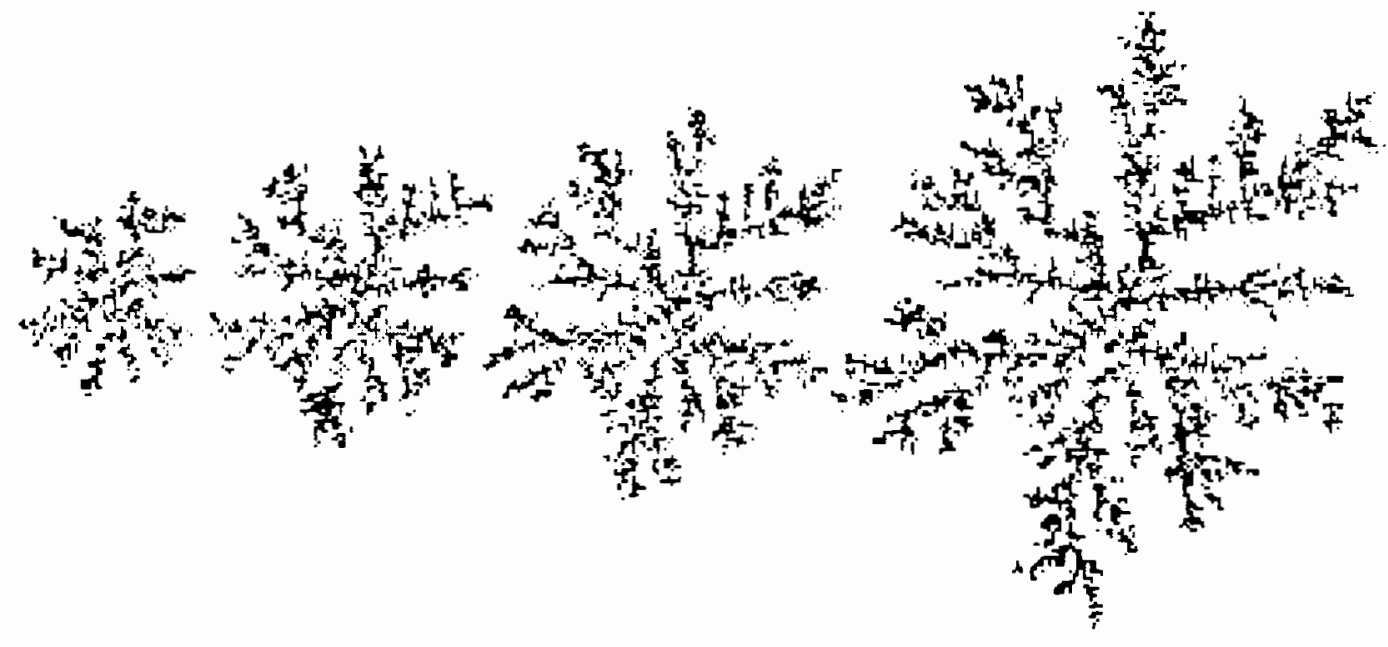

$25 \% \quad 58 \% \quad 18 \% \%$

Figure 7. Quartile stages of growth of aggregate number 20. 
Because the deposit's diameter, lattice spacing, and step size, prior to deposition, are identical, it is improbable that any periodicities in the $X$ and $Y$ directions would be detected in the histograms for these coordinates.

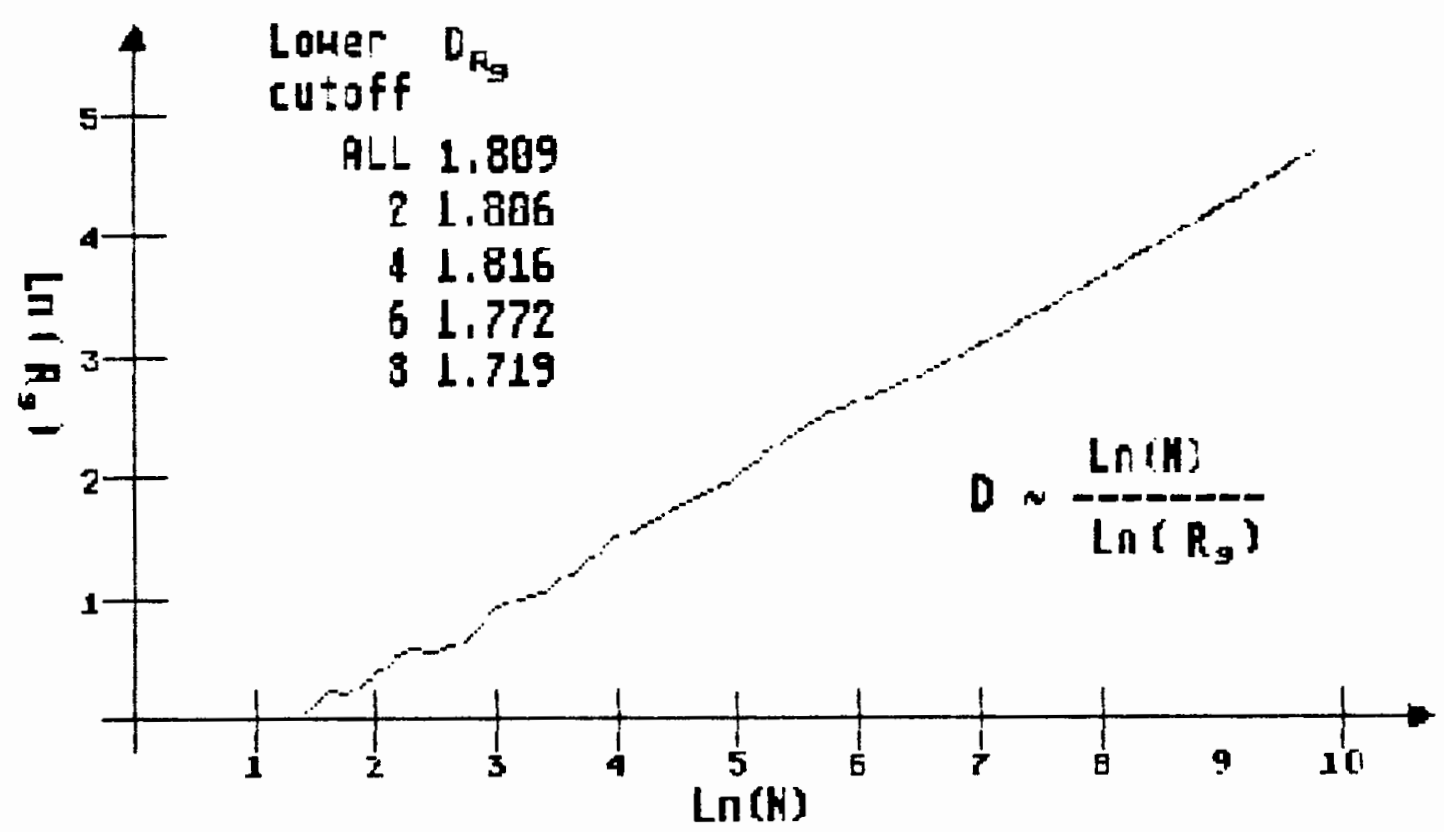

Fiqure 8 . Radius of gyration dependence on number of deposits for aggregate number 20.
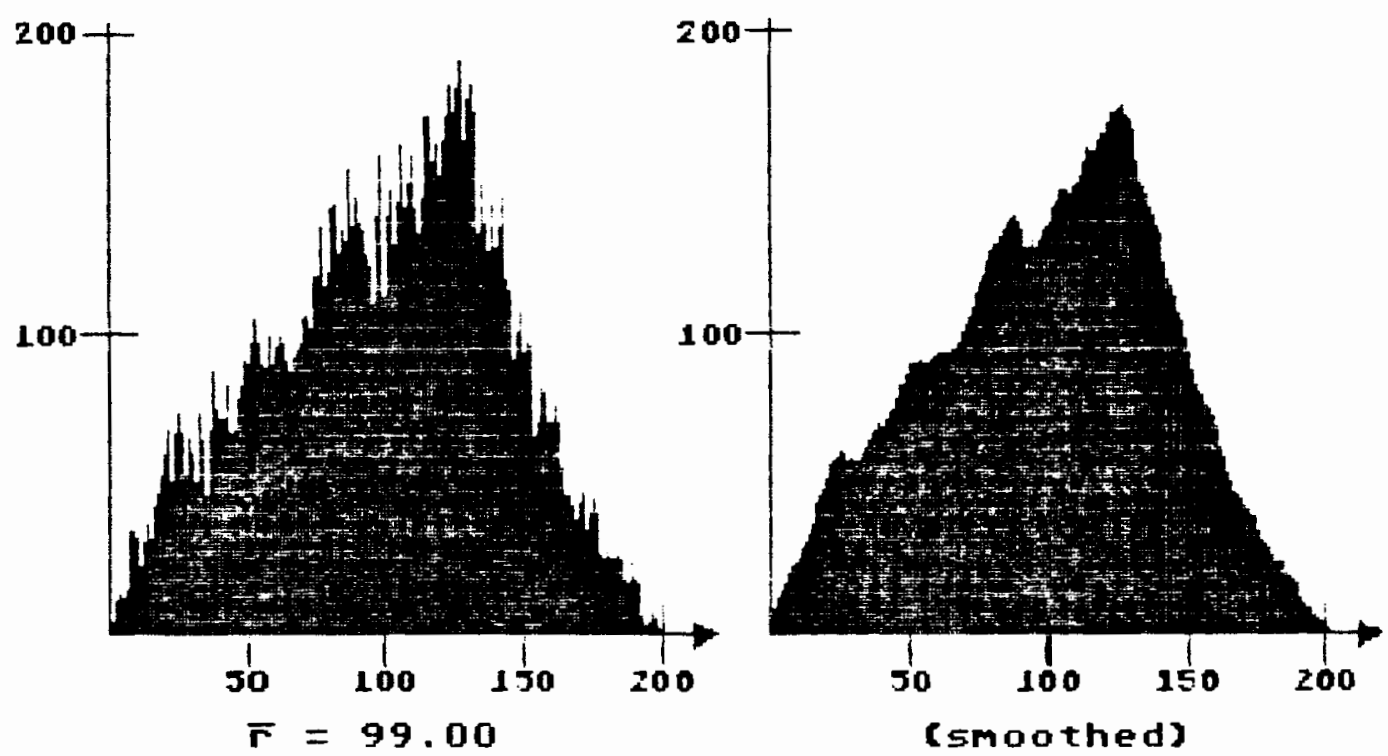

Figure 9. Radial mass distribution for aggregate number 20. 
These distributions, presented in Figures 11 and 12 , are not uniform due to the interaction between the arms and the deposition process. (Comments concerning the averages of these distributions are presented below under the discussion of the cumulative distribution of the large aggregates.)

The effect of screening on the growth is depicted in Figure 13. The ultimate $N \%$ of the total deposits are illustrated, for $N=10, \ldots, 90$. On the average, the deposition occurs in the outer and more active shell. However, occasionally, screening is incomplete and a random walker wanders deeply into a "fjord" before coming to rest,

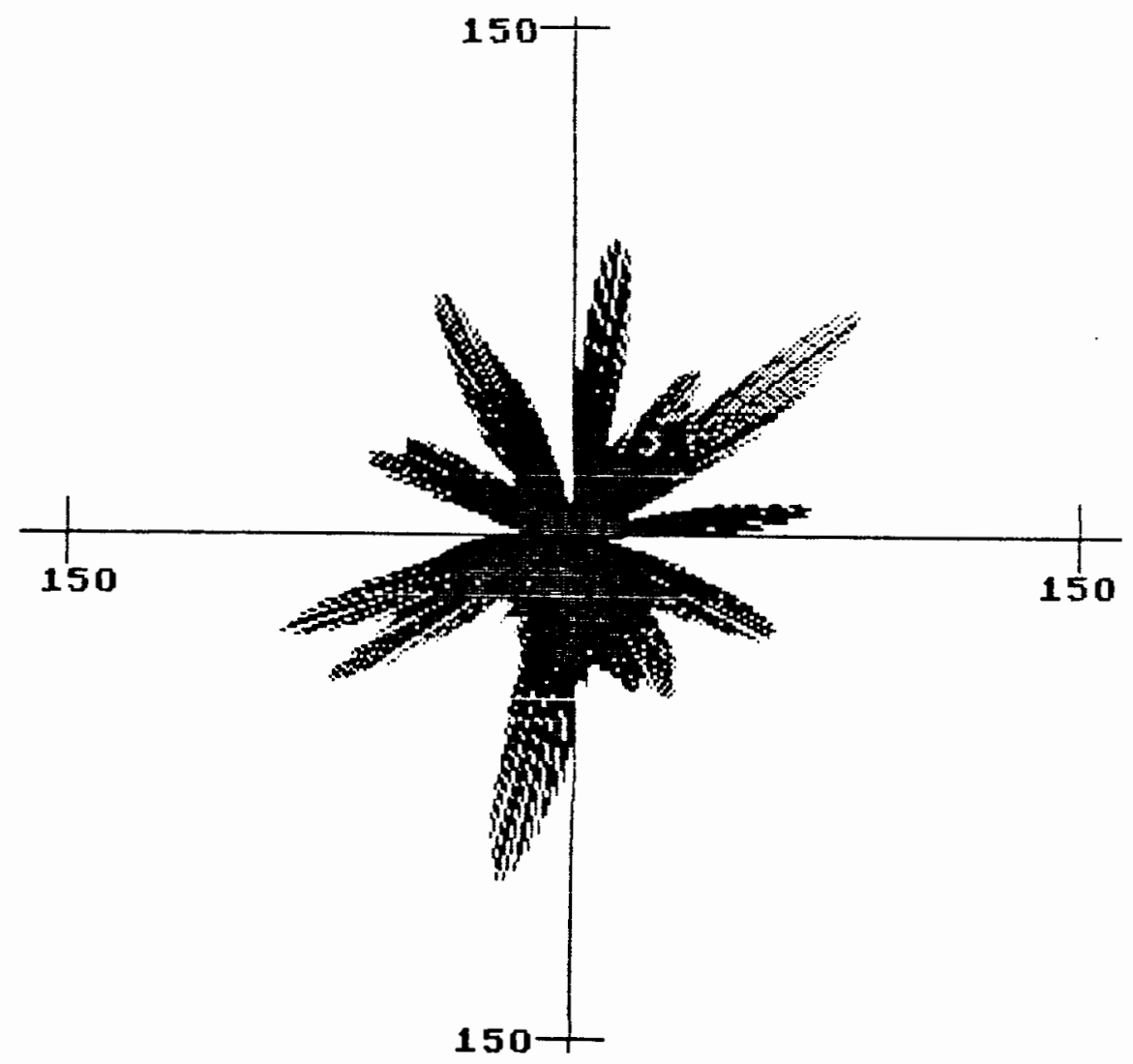

Fiqure 10. Angular mass distribution for aggregate number 20 (smoothed). 
as shown by the stray deposits which have penetrated the interior. This screening process limits the "filling in" of the interior, and growth continues in the outer shell. Subsequently, this active shell extends, by virtue of the deposition occurring there, leaving behind the incompletely "filled in" interior of the aggregate, which is a fractal, rather than a compact structure.

Figure 14 examines the sample space of the cumulative probability distribution of the large aggregates for uniformity and isotropy of deposition. The suggestion of underlying arms, most discernable in those images labeled

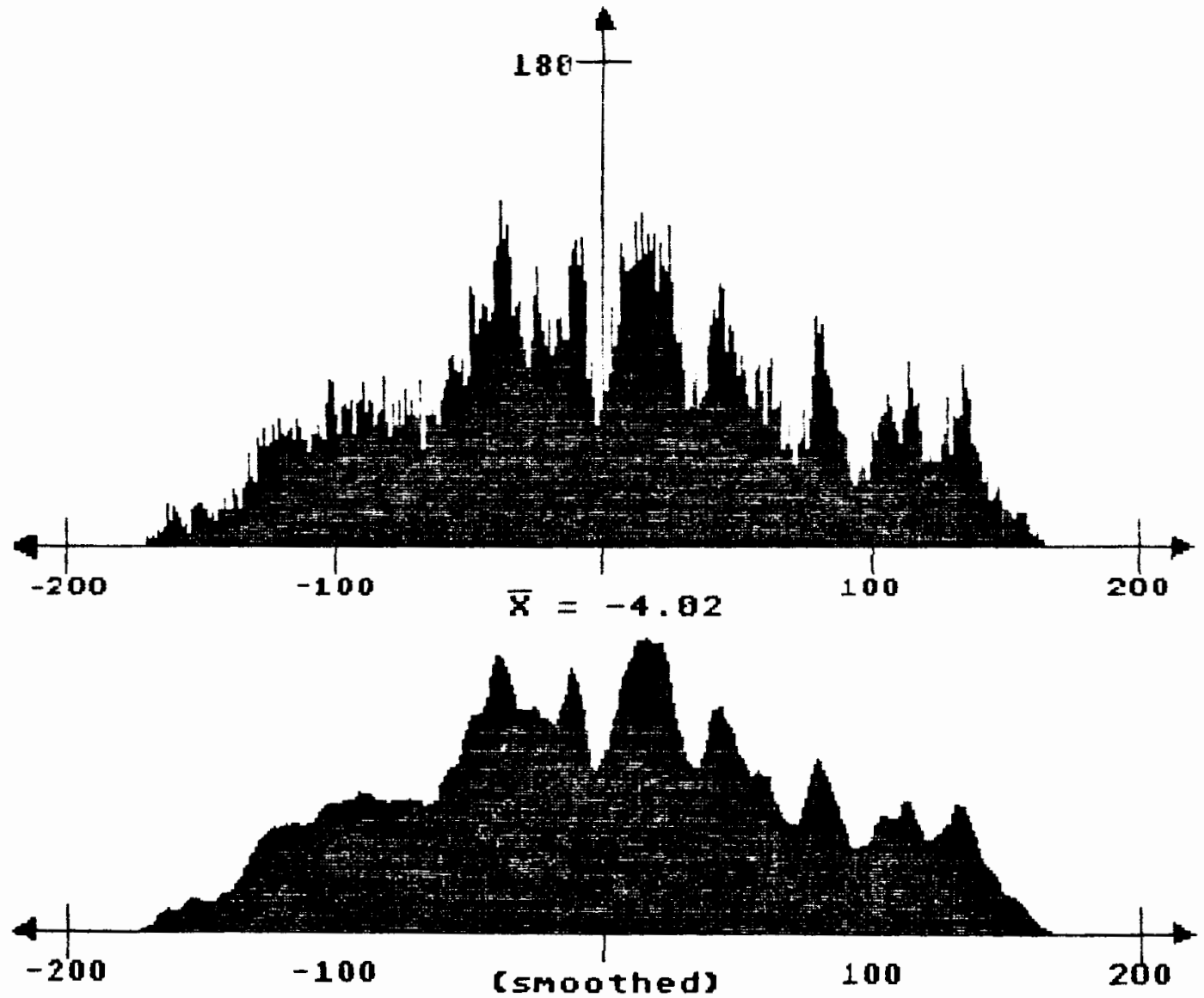

Fiqure 11. Mass distribution in $X$ for aggregate number 20. 
$30 \%$ and $40 \%$, (which are projections of the deposition distribution onto the $X Y P l a n e$, for $P(\underline{X}) \geq .30$ and .40 ) and the corresponding modes in the angular mass distribution of the 1 arge aggregates, which is presented in Figure 15, could be an effect of the lattice, if deposition was most probable along the orthogonal and diagonal directions of the lattice. Moreover, there does not appear to be any pattern associated with those sites which have not been deposited, except that they tend to be between those arms. The averaged growth appears to be uniform and radial because the perimeters of Figures 14 and 15 can be regarded as circular.

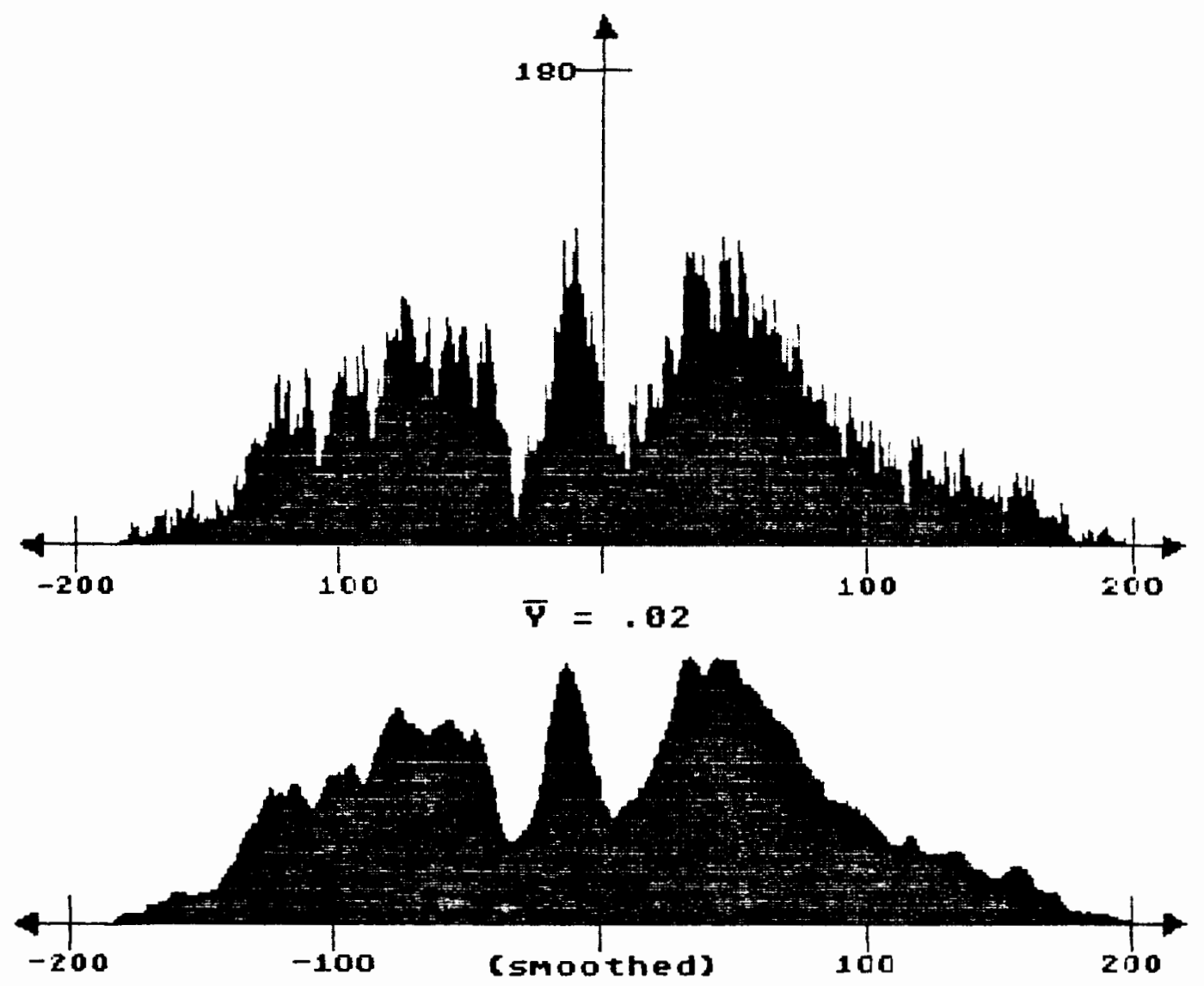

Fiqure 12. Mass distribution in $Y$ for aggregate number 20. 


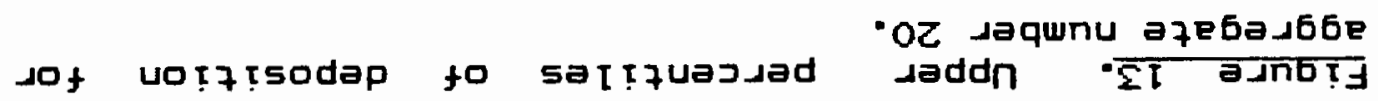

\section{$\% 06$}

\%88

$\%$ B

at

* int

trit

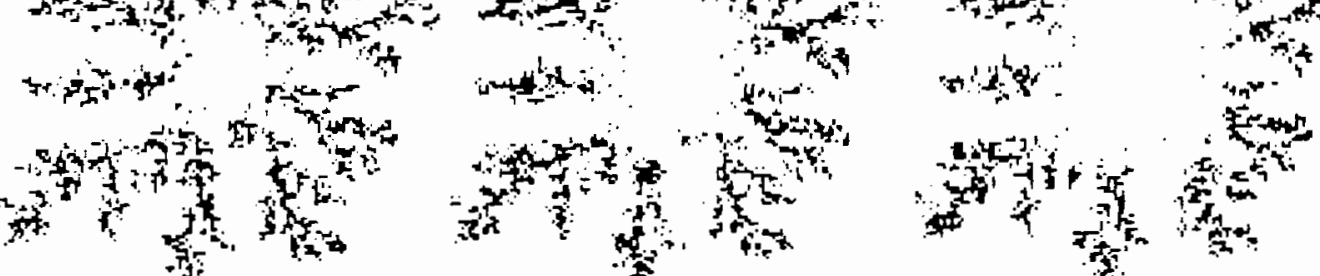

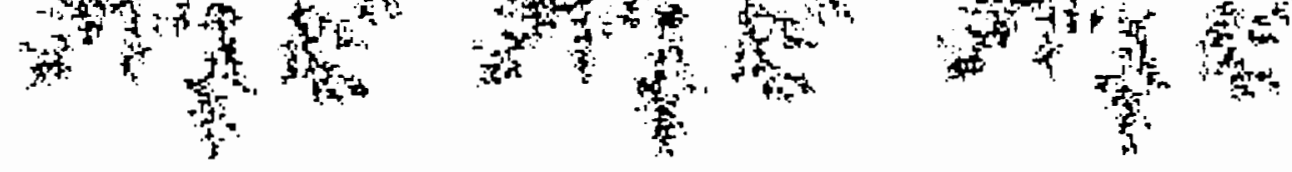

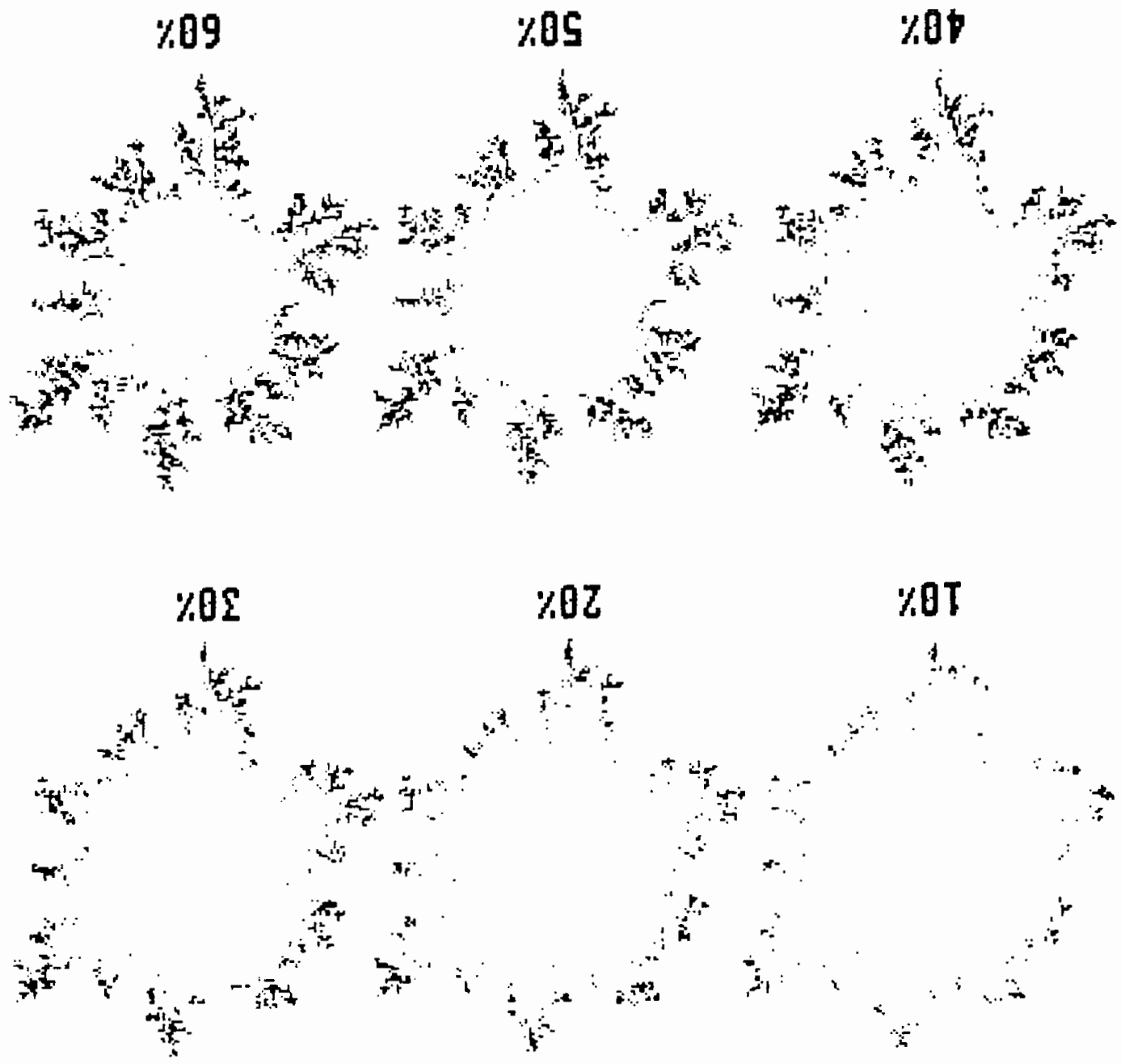


The frequency histograms for the cumulative distributions in $X$ and $Y$ are displayed in Figures 16 and 17 , respectively. The center of deposition is located at (3.47,-5.37). The center is 6.4 lattice spacings from the origin of the simulation. This result exposes a possible source of error in the fractal dimension based on the radius of gyration and is discussed at length in the Conclusion and Appendix D. Factors which might influence the displacement of the average center of mass, as accumulated over the

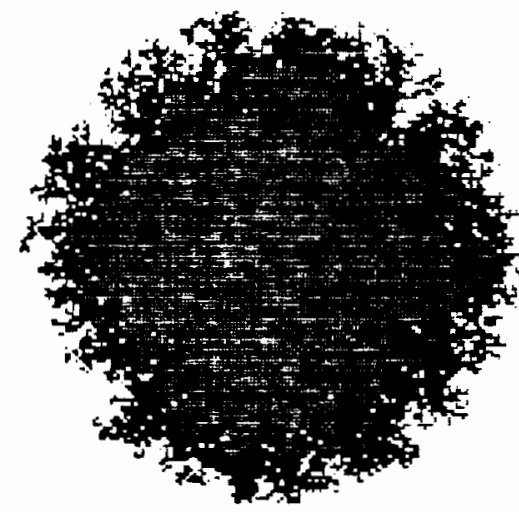

Qj.

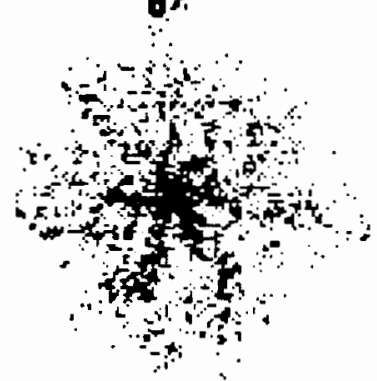

$30 \%$

E. B.:

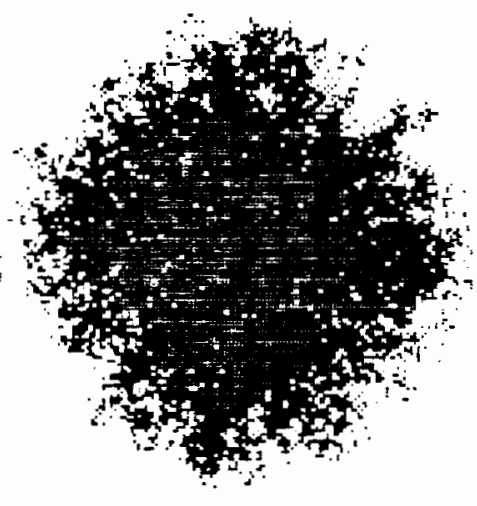

$10 \%$

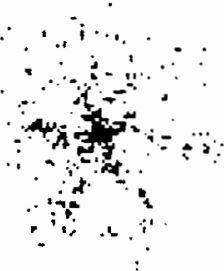

407

$+$

$70 \%$

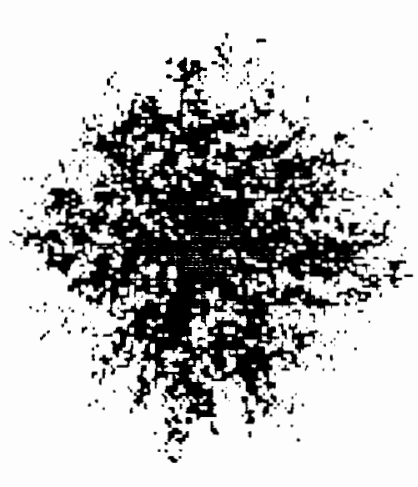

$20 \%$

$50 \%$

Pixels displayed represent sitcs with deposition probability greater than or equal to the indicated percentage.

Fiqure 14. Cumulative probability distribution in $X$ and $Y$. 
relatively large sample of aggregates, are that the incident flux is not isotropic, that the deposition is preferential to certain orientations, or that growth is restricted in some directions. (The center of mass for any particular aggregate is expected to be displaced.) Because the graphics screen was dimensioned by even, and not odd integers, the lattice origin was slightly eccentric to the screen boundaries. Consequently, growth was terminated slightly more often when the maximum radius was in the fourth quadrant. However, this would explain the location of center of deposition in the second, and not in the fourth quadrant. Possibly, this asymmetry was caused by nonuniformity of the random number generator function. If it was biased towards higher values, the "birthing" circle would have released a greater flux of random walkers into the fourth quadrant. Unfortunately, time did not allow for analysis of the random number generator. (This bias also would have caused anisotropy in the Brownian motion, which could have countered the above effect, because the leeward side of the aggregate would have obstructed movement and collected more deposition. However, not knowing the shape of the random number distribution, it is impossible to predict how the "jump" procedures, which direct the movement, would have responded to the anisotropy.) The radial symmetry is indicated by the joint symmetry in $x$ and Y, as shown in the histograms. 
The frequency histograms for the radial distribution of the large aggregates, shown in Figure 1B, are included for comparison to Figure 9. Because uniformity of deposition would imply that the aggregates would not be fractal, it is not to be expected. If the large aggregates are fractal, then the increasing portion of the histogram should exhibit power law dependence, specifically, rD. That it departs from this is most probably due to occasional penetration into the interior. The decreasing portion of the histogram indicates that growth is incomplete and possibly that the active zone of deposition has different

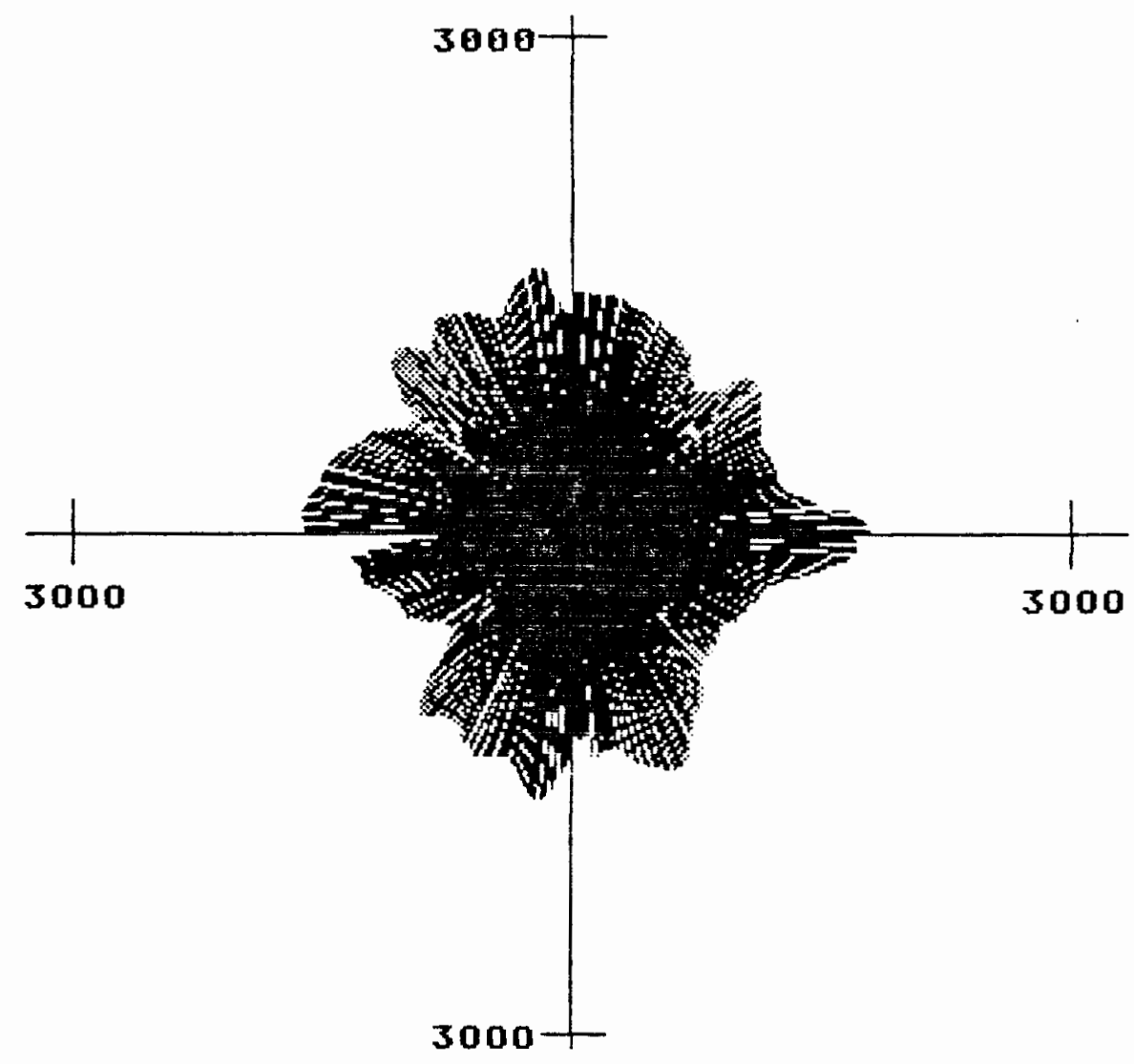

Fiqure 15. Cumulative angular mass distribution of the 30 large aggregates (smoothed). 
scaling properties than the more complete interior region. However, its decreased inclination, as compared to Figure 9, is most probably the result of the averaging which occurred when the histogram was constructed from a composite of all the large aggregates.

Figure 19 depicts the dependence that subsequent growth has on initial conditions. The large aggregates, numbers 23 and 27, were each grown from the small aggregate, number 23. Even though the large aggregates are more than three times the size of the seed aggregate, the small aggregate seems to have imparted a general growth trend.

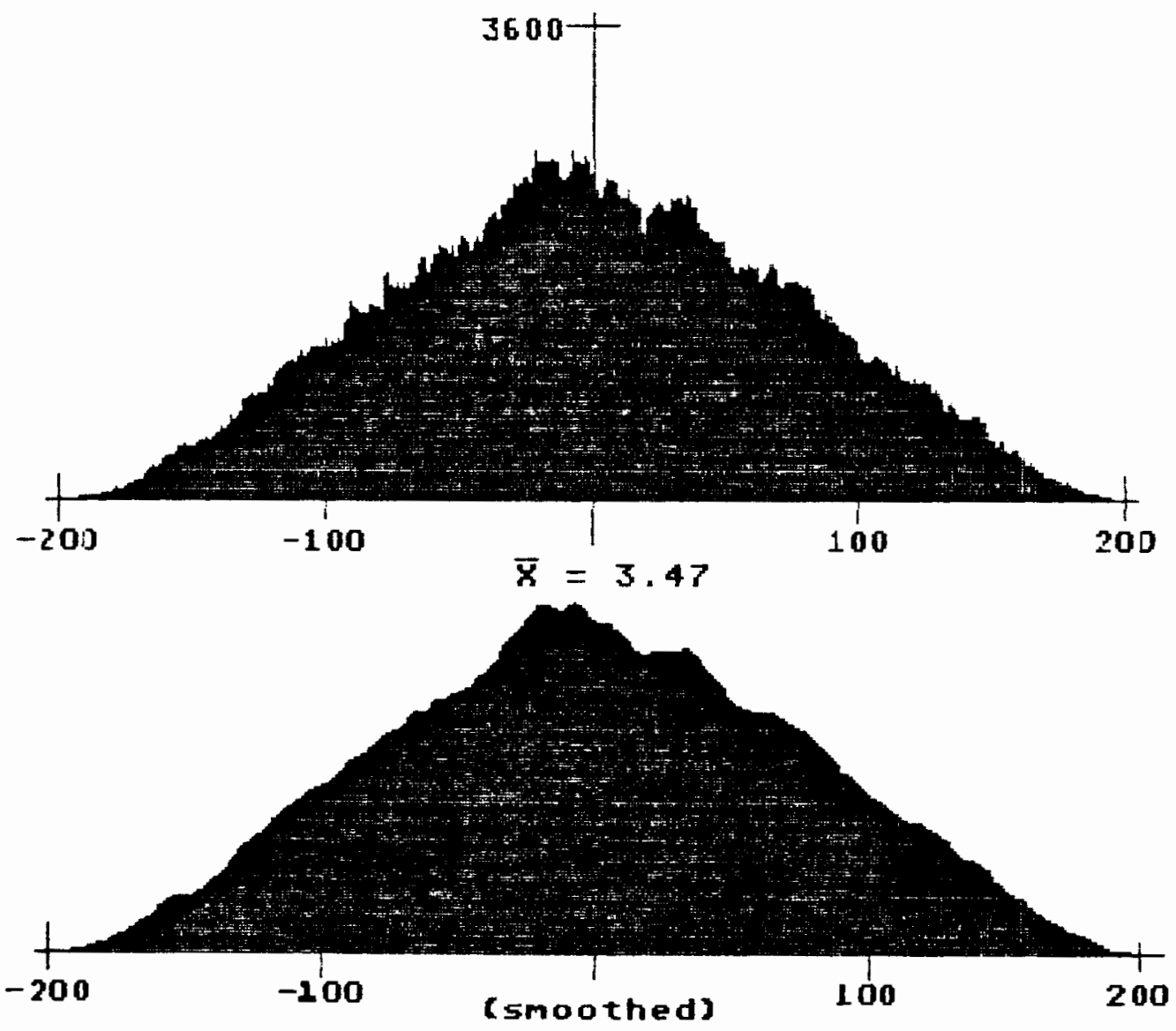

Fiqure 16. Cumulative mass distribution in $X$ for the 30 large aggregates. 
This similarity of structure between the two large aggregates persisted, even into regions beyond the scale of the original aggregate. The large aggregates were grown to sizes of 16464 and 19056 deposits, respectively. An investigation of the divergence of their morphologies with further growth was not performed.

All of the small aggregates were grown from a single featureless seed. Yet, each of the aggregates developed distinctly, with its own characteristic structure. The

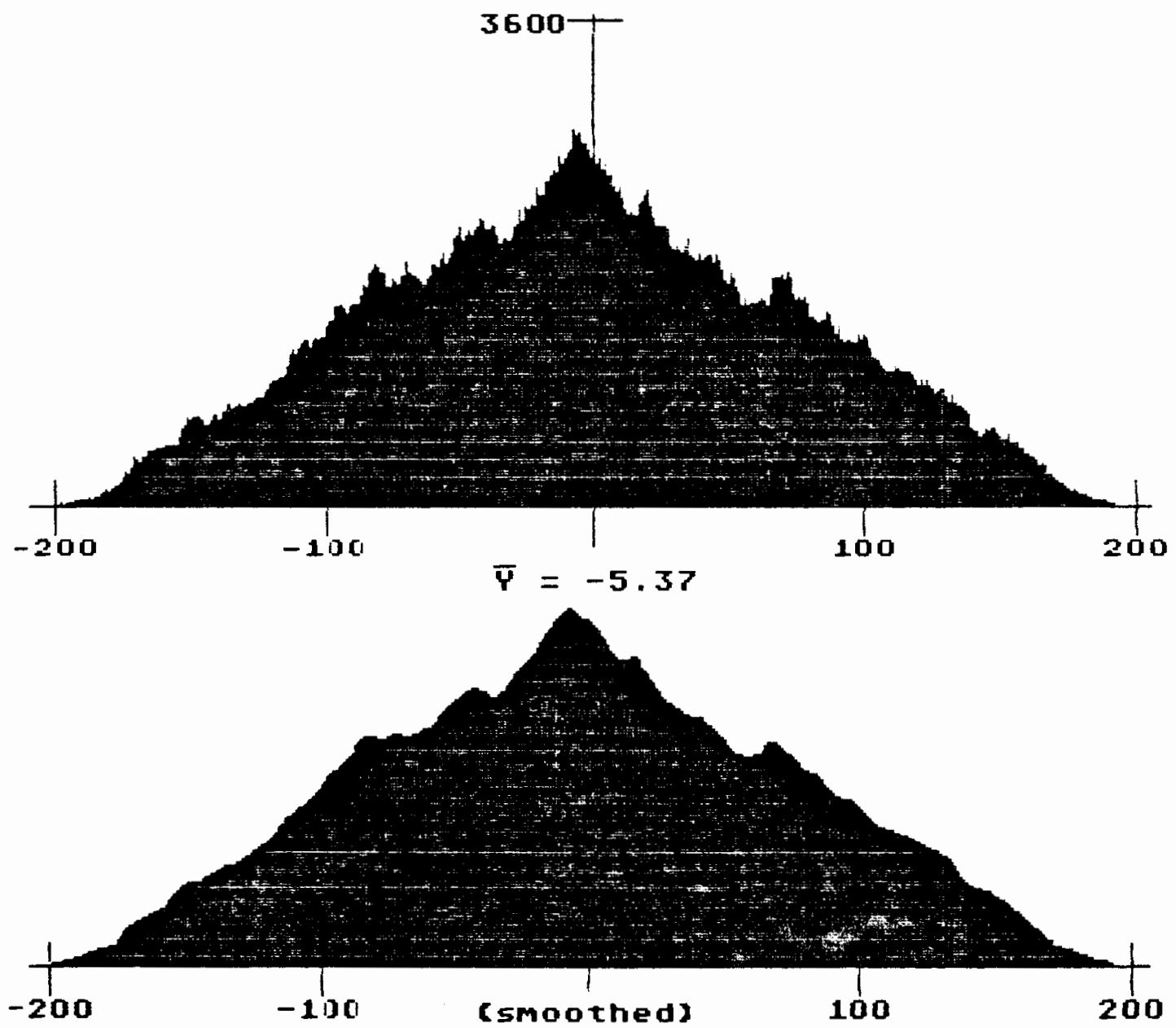

Fiqure 17. Cumulative mass distribution in $Y$ for the 30 large aggregates. 


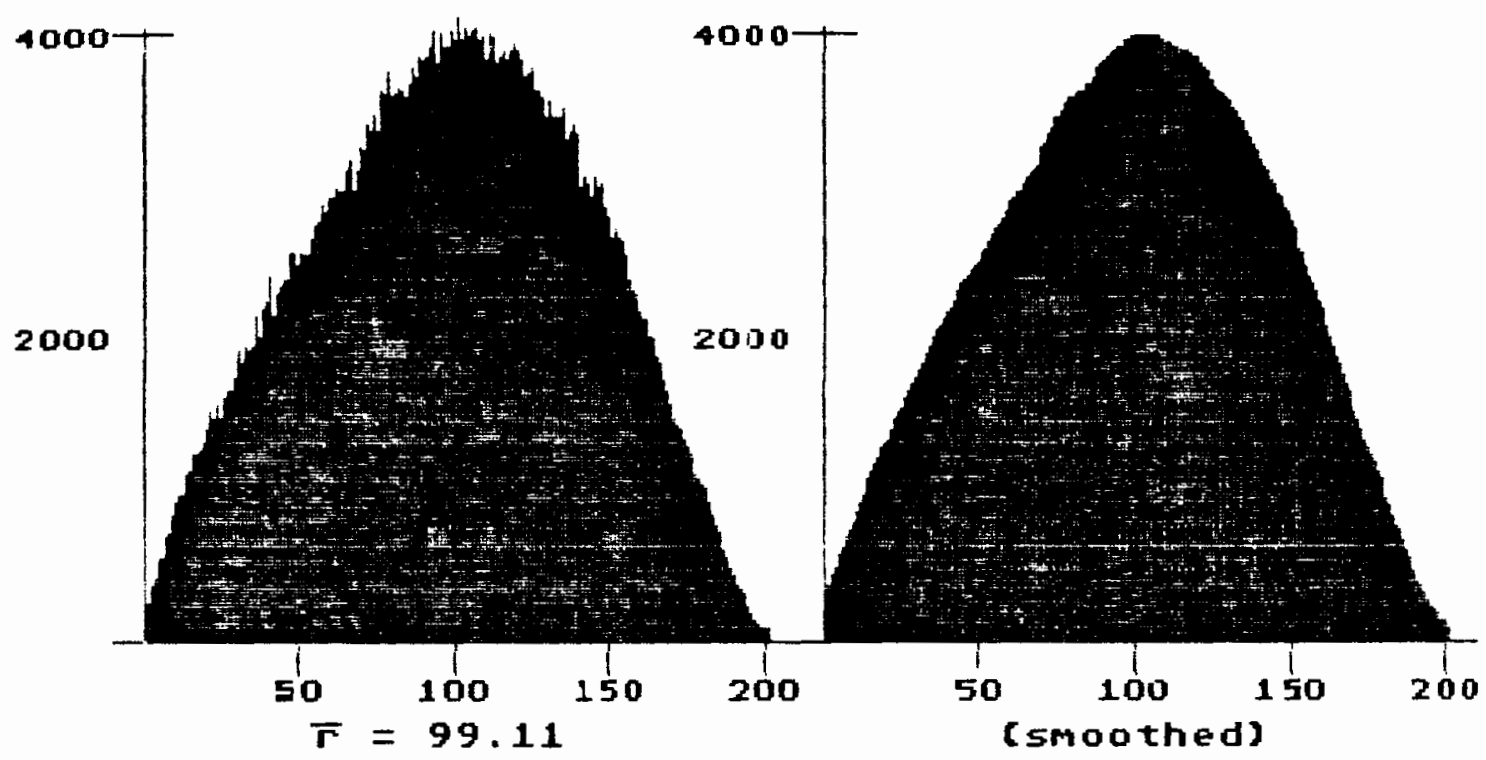

Fiqure 18. Cumulative radial mass distribution for the 30 large aggregates.

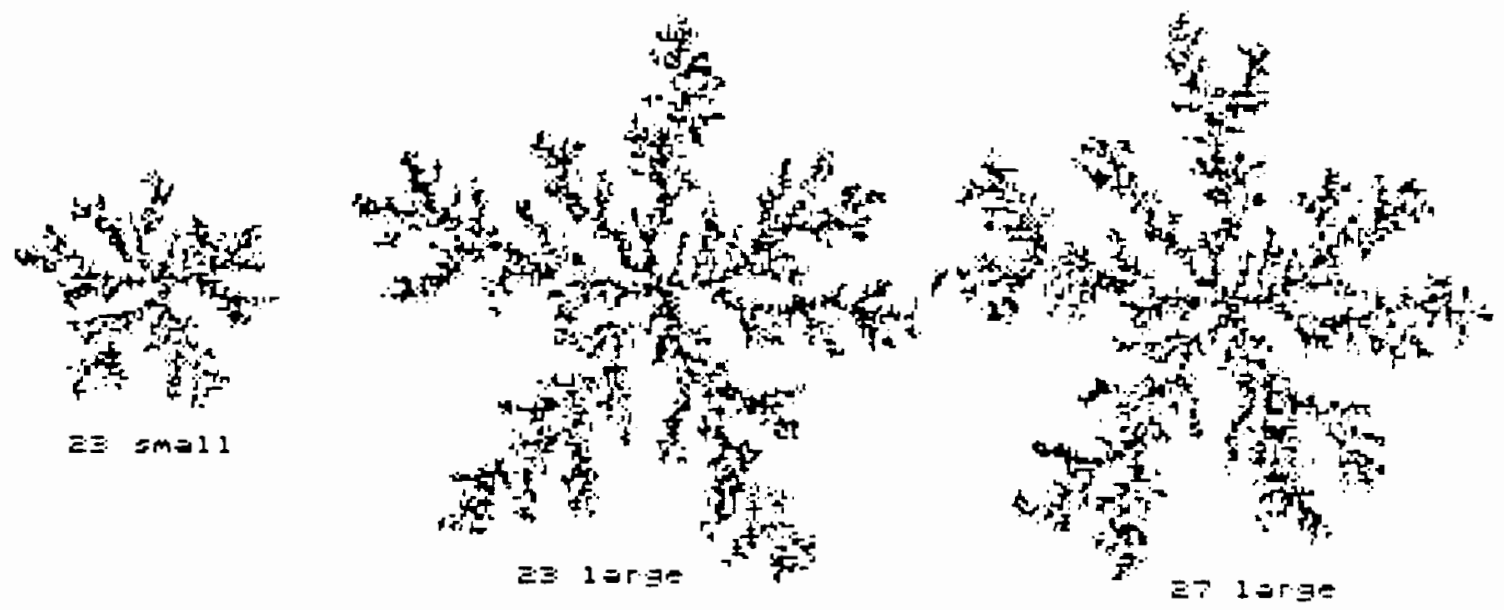

Fiqure 19. Persistence of growth trends.

fractal dimension not only describes how its density scales, both locally and globally, but also the resemblance noticeable in those characteristic structures due to the scale invariance, or self-similarity. 


\section{CHAPTER $V$}

\section{CONCLUSION}

The aggregates were grown by a random process yet their structure is not entirely random. Their structure is symmetric under changes of scale, from lengths of a few pixels to that on the order of the size of the aggregate itself. A consequence of their self-similarity for scaleinvariance of their patterns) is that their density decreases as their size increases. By contrast, a two dimensional Euclidean disk with homogeneous mass density, which is compact within its perimeter, has constant density regardless of its size. Consequently, as the density of a fractal aggregate decreases to zero the perimeter becomes infinite. (Another formularization for the fractal

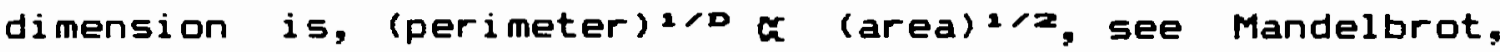
1983.) The ramification of the structure of an aggregate contributes to this increase in the aggregate's perimeter. The screening effect which causes the arms to grow out more than interior to fill in, contributes to the decrease in density. The diffusion-limited aggregation mechanism operates on the microstructure using local growth rules, the effects of which are mediated through the fractal property of self-similarity and affect the resulting 
macrostructure.

Mass/length scaling relationships associated with the aggregates were analyzed to obtain a measure of the fractal dimension. The dependence of the radius of gyration on aggregate mass yielded a dimension related to global properties of the aggregate while the density-density correlation function gave a dimension more associated with local properties. The agreement between these two methods is due to the fractal property of scale invariance. The various modifications of the correlation function indicated that the shape of the correlation "window" is not pertinent to the evaluation of an aggregate with radial symmetry and which is grown on a square lattice. However, the results given by the method using both square "windows" and the inclusion of the edge, more quickly attained the value to which the results of the other methods appeared to converge, as the average size of the aggregates increased. It should be noted however, that the method which would have used exactly circular "windows" together with inclusion of the edge was not performed so that this value could be due to only the inclusion of the edge, independent of the shape of the 'window'. The methods which excluded the edge did provide additional information about the screening effect. Furthermore, the results of these methods which utilized square 'windows' and circular 'windows' did not differ significantly. The fractal dimension as calculated over the 
entire aggregate essentially remained constant as the size of the aggregate increased. When the edge was excluded from the correlation analysis, the correlation function indicated that the interior of the aggregate had a greater fractal dimension than the entire aggregate. However, the interior did not become compact indicating that the outer edge was screening the interior. (See Appendix E for possible modifications of the edge analysis.) The fractal dimension using the correlation function is $D_{C}=1.67 \pm .01$.

After finalizing the analysis and discussion of the graphical results, it became evident that the offset in the location of the center of deposition from the lattice origin was, in fact, appreciable. Consequently, the approximation used in the radius of gyration calculations was not justified and the results had a systematic error. This offset, L, enters into the radius of gyration calculation in a complicated manner. Although, utilization of the parallel axis theorem could correct the radius of gyration for each deposition, $N_{\text {s }}$ it would require the functional dependence, $L(N)$. However, the dependence that the offset has on $N$ is non-trivial and depends on the interaction of the growing structure with the random mechanisms of the simulation. Further discussion of the approximations used in the recalculation of the fractal dimension based on the corrected radius of gyration is given in Appendix D. It is noted there that the concavity in the graphs, mentioned 
above, may be due, in part, to this error. The error, also indicates that "radius of gyration", as measured from the lattice origin, is not as characteristic of the aggregate as the true radius of gyration. The fractal dimension based on the radius of gyration dependence is, $D_{\text {no }}=1.75 \pm .08$.

The correlation function results using 'windows' of 1.5 to 32.5 lattice spacings of $1.67 \pm .01$ are in agreement with the accepted results of $1.68 \pm .05$, as reported by Meakin (1983b), where "windows" of 5 to 50 lattice spacings were utilized. The radius of gyration results of $1.75 \pm .08$ are in precise agreement with the accepted results reported there.

The differences with Meakin's model do not give significantly different numerical results. The slight difference in the boundary conditions, which might allow pixels to more completely fill cavities with entrances of one pixel in diameter, could give slightly different graphical results. The aggregates could be analyzed for the presence of "lakes", which would indicate that occasionally a pixel could close off the opening of a "fjord". However, this analysis was not performed, in part, because Meakin's graphical results were not available.

The graphical results demonstrated the diversity in the morphologies of the aggregates as well as the symmetry property of self-similarity. The animation programs clearly demonstrated the decreasing penetration into the interior of 
the aggregates by the random walkers as the aggregates grew larger. The perimeter of an aggregate screens the interior and grows preferentially. Intricacies in the perimeter are enhanced by the growth mechanism and tend to be extended. Thus, the patterns of the large aggregates resemble the patterns of their predecessors.

The morphology of a diffusion-limited aggregate resembles the fractal structures of those physical processes such as electrodeposition and fluid-fluid displacement. The measured fractal dimensions for these processes, as previously stated in Chapter II, are 1.66 and 1.70, respectively. This supports the contention that diffusionIimited aggregation belongs to the same universality class of physical behavior. 


\section{REFERENCES CITED}

Brady, R. and R. Ball. "Fractal Growth of Copper Electrodeposits," Nature, 309, 225, (1984).

Daccord, D., J. Nittmann, and H. Stanley. "Fadial Viscous Fingers and Diffusion-Limited Aggregation: Fractal Dimension and Growth Sites," Phys. Rev. Lett.. 56, 3.36, (1986).

Forrest, S. and T. Witten. "Long Range Correlations in Smoke Particles," J. Phys. A: Math. Gen., 12, L109, (1979).

Langer, J.. "Instabilities and Fattern Formation in Fattern Growth," J. Rev. Mad. Fhys., 52, 1, (1980).

Mandeltrot, B.. The Fractal Geometry of Nature, (Freeman, New York, 1983).

Matsushita, M. M. Sano, Y. Hayakawa, H. Honjo, and Y. Sawada. "Fractal Structures of Zinc Metal Leaves by Electrodeposition," Phys. Fev. Lett., 53, 286, (1984).

Meakin. P.. "Diffusion-Controlled Cluster Formation in Two, Three, and Four Dimensions," Phys. Rev. A, 27, 604, (1983a).

Meakin, P.. "Diffusion-Controlled Cluster Formation in 2-6 Dimensional Space," Phys. Rev. A, 27, 1495, (1983b).

Niemeyer, L., L. Pietronero, and H. Wiesmann. "Fractal Dimension of Dielectric Breakdown," Phys. Fev. Lett., 52, 1033, (1984).

Paterson, L.. "Diffusion-Limited Aggregation and Two-Fluid Displacements in Porous Media," Phys. Rev. Lett., 52, 1621. (1984).

Witten, T. and L. Sander. "Diffusion-Limited Aggregation, a Kinetic Critical Phenomenon," Phys. Rev. Lett., 47, 1400, (1981).

Witten, T. and L. Sander. "Diffusion-Limited Aggregation," Phys. Fev. B, 27, 5586, (1983). 


\section{APPENDIX A}

\section{THE COMPUTER PROGRAMS}

The selection of this thesis topic was, in part, motivated by the desire to demonstrate the feasibility of performing credible physics research on a personnel computer. Many student researchers do not have access to mainframe computers, especially those with graphics capabilities. Although, it could be said that fractal geometry is one of the computer viruses of the $1980^{\circ} \mathrm{s}$. The computer programs developed in this project can serve as a basis for further research by students interested not only in the fractal patterns they generate, which resemble many patterns found in nature; but more importantly, by the apparent generality of the model to natural and technological processes.

Initially, the simulation was attempted on a Commodore C-64 computer as it was a very popular and inexpensive system. However, with only $64 k$ bytes of random access memory, a slow (1Mhz) 8 bit microprocessor, small maximum array size (32k), and a graphics screen of only 320 pixels by 200 pixels at 'high' resolution, it was abandoned as soon as larger and faster machines became available. The Atari $10405 T$ was selected because it had the most advanced 
technology at that time (1986), although, since then it has been superseded by other systems, preferred by researchers, because these systems are more technically supported.

The Atari 1040ST with its $16 / 32$ Motorola 68000 microprocessor operating at 8 Mhz with 1 Megabyte of random access memory is still a respectable system. However, the basic language interpreter supplied by Atari had 'bugs' in the integer arithmetic routines and could not even use $32 k$ of memory for arrays. With this memory limitation, simulations could not be done which would realize the potential of the 640 pixels by 400 pixels graphics display. Fortunately, GFA Basic was developed by GFA-Systemtechnik (which has become the system standard for the Atari, especially in Europe, where Atari is on par with IBM or Apple computers). The following computer programs were written in GFA Basic version 2.0.

The following short demonstration program was the prototype of more complicated and extensive programs and is included, with comments, to offer insight into the structure and coding of the simulation. It models DLA in a toroidal geometry on a two dimensional square lattice. The simulation space is a 400 by 400 lattice. The deposits are stored sequentially in an integer array using ten bit packed words; at the termination of the program the core image is dumped to a binary sequential file on disk. 


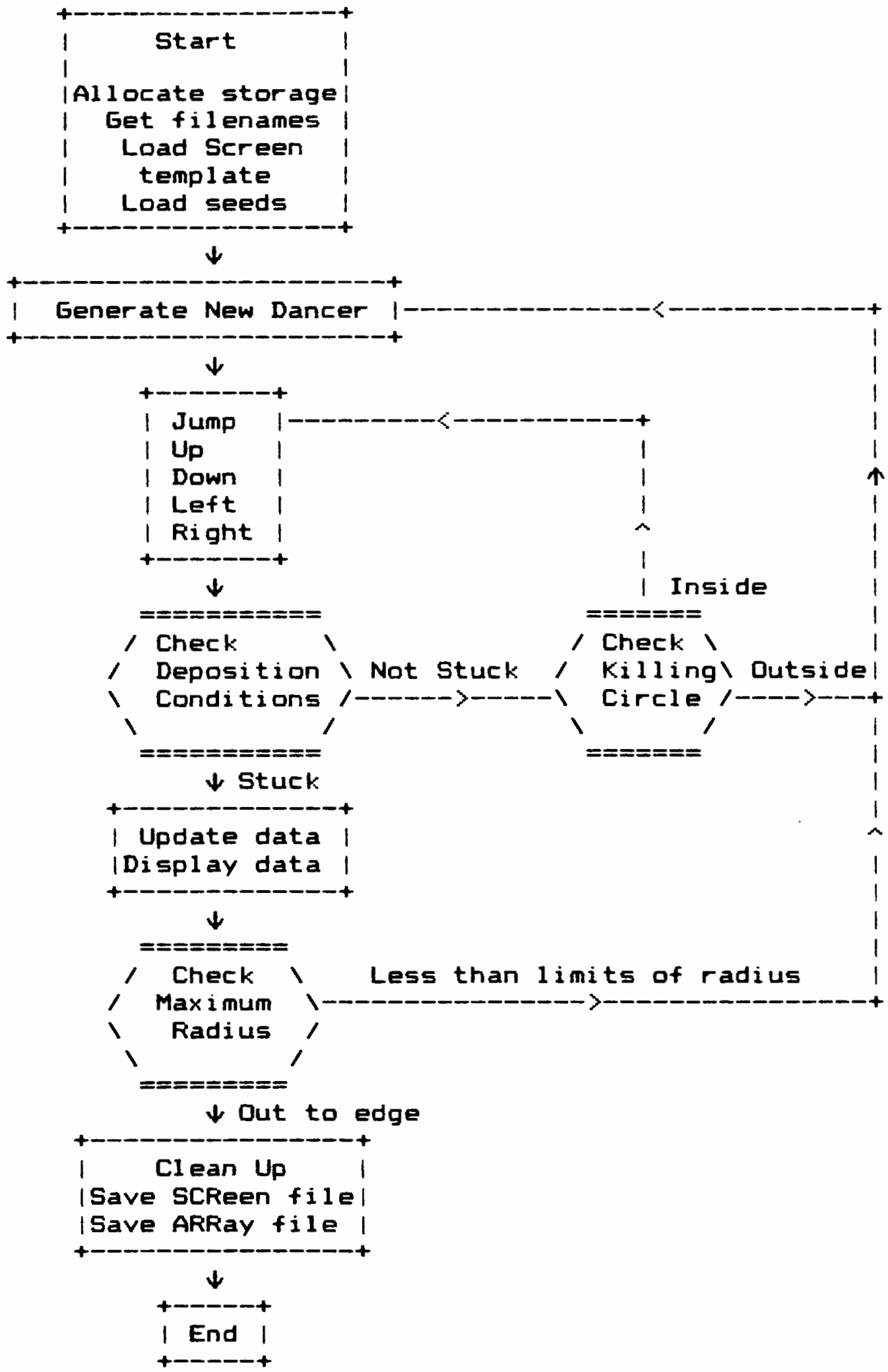

Fiqure 20. Demonstration program flowchart. 
Cls 'Clears the screen.

Grapheode?

' 3 is complesent oode, 50 plot $(x, y)$ alternately sets and clears $(x, y)$.

Deftext 1

' Standard text aode for Text cosoand.

Color 0

- Plot color is white (for white dot on black background).

On Break 6osub Breakhandler

- Control-Shift-Reset vectors through this cleanup routine.

On Error Gosub Errorhandler

' Any errors vector through this cleanup routine.

Print "Starting̣ seed filenane:"

Fileselect "M.SCF", "SEED.SCR", As

' Selects a filenane for NULL for nene) to act as the seed.

Print At $(1,1) ; "$ "Storage filename:

Do

Fileselect "( 1 , SCR", Hids (As, 2), 8s

' Selects filenase to save work.

Exit If 8 s $>$ " And Bs (\rangle$\left.^{\prime \prime}\right) "$

' Won't accept null filenases, a place is needed to save work:

' Loops until a vaild filenane is obtained.

Loop

If Instr (85, "SCF") $=0$ Then

'If the SCReen extension isn't there...

If Instr (B\$, " $")=0$ Then

"checks for a period;

$B s=B S+$ ".

'adds it if it's not there.

Endif

$B s=E s+{ }^{2} S C F^{2}$

'then adds SCKeen extension.

Endif

Hides

Din Ordery $(30000)$

- Allocates storage for the array of deposit coordinates.

Order $h(0)=1$

' (0) is location for the nuaber of deposits, $n=(0)+1$, since $(0)$ and $(1)$ are occupied.

- That is, first deoosit is in Orderq(2).

Order $\%(1)=0$

' (1) is the saxious radius of the growth fron the center of the screen.

If $A \$="$ "Or $A \$="$ " Then

'If 'CANCEL' was selected for "Starting Seed", then sets up standard screen.

$\mathrm{Cls}$

Deffill 1,1

- Sets fill as solid black, and

Fill 320,200

' fills it up froe the center out.

Plot 200,200

- Starting point (seed).

Order: $(2)=205000 ! 205000=200 ! 1024+200$

Order $\%(0)=2$

- Put the seed as the first eleaent of the array. 
Line $400,0,400,400$

' Right boundary.

Line $401,301,639,301$

- Dividing line beween title and data sections.

Text 408,16. "Sinulation of Diffusion-"

Text 408, 32, "Linited Aggregation by"

Text 408,48, "single particle eigration."

Text 408.64,"Diffusion space: 2-D planar"

Text 408,80," square lattice"

Text 408,96. "Deposit 5pace: 2-D planar"

Text 408,112," souare lattice"

Text 408,128: "Trajectories:"

Text 408,144," collision layer; unit steps"

Text 408,160," diffusion zone: or thogonal"

Text 408,176," steps; scaled to $R^{*}$

Text 408,192," $(R=$ axiau radius; dynanic)"

Text 408,208,"Initial seed: central pixel"

Text 408,224," "Eenerating geonetry: circle;"

Text 408.240," radius $=R+5 "$

Text 408,256, "Killing geosetry: annulus;"

Text $408,272, "$ siniaus radius $=2 R+5$ "

Text 408, 288," Sticking probability $=1.0$ "

- Data section of screen starts here:

Text 408,316, "Deposits:"

Text 408,332, "Maxicue grouth radius:"

Text 408,348 , "Angle of maxinue radius:"

Text 409,364, "Data on Last Dancer"

Text 408,380, "R: $\quad \theta:$ :

Text 408,396, "Nuaber of jups:"

Else

' Else if a filenane was selected for a seed, load the

Bload As, Xbios (2)

- screen portion into the screen eesory and the

Bload Lefts (As, Instr (As,".") +'ARG", Lpeek (Arrptr (Order\% (1))

' array portion into the previously allocated array.

Endif

Jupp: $=1$

- Junp\% is the nuaber of spaces a dancer can jusp, depending on how close it is to the deposition zone Njuaps $=0$

' Nuapst is the nubber of junos dancer (s) have ade since last depostion.

Do

' Main loop of progran. Loops until deposit reaches the edge.

Stuck=False

' Starts out with dancer unstuck. so it can oove.

Juapl=1

Gosub Mewdancer

- Generates a new particle.

Repeat

- Actual dancing loop. This akkes the dancer sove.

Xold $d y=x y$

Yoldy=Yy

' Saves old location of dancer for conparison, 
' or to leaves particle there if deposition conditions are satisfied.

On Rando (4)+1 Gosub Up, Down, Left, Right

- Randor nubber 1 through 4,1 goes up, 2 down, etc.

Inc Nuaps\%

- A juap was aade, so count it.

On Juap\% Gosub Check

- If Jupp:=1 (ie. in depostion zone! then checks deposition criteria.

If Not Stuck Then

- If the criteria was not set then

Plot Xold\%, Yold\%

"erases the old dancer pixel.

Plot $X \%, Y \%$

' and draws the new one at the new coordinates.

Endif

Rdy $=$ Int (Sar $\left.\left((x y-200)^{\wedge} 2+(Y y-200) \wedge 2\right)\right)$

'Calculates the distance fron the center of the deposit.

If Roy $>210$ dery $(1)+5$ Then

'If the dancer gets outside the killing circle at 2 kisax $+5 \ldots$ Stuck $=$ True

"artificially sticks it (so it gets replaced with a new dancer)

Plot $X Y, Y y$

' and erases it froa the screen.

Endif

If Rdy $>$ ordery $(1)+5$ Then

- If outside depostion zone, scales the junoing distance; larger jusps will econonize run tiee. Juep $\%=2^{\wedge} \operatorname{Int}(1.442695 t \log ((\operatorname{Rd} \%-0 \mathrm{rder} \%(1)) / 5))$

El se

Jueph $=1$

' Inside the deposition zone, jusping is single-stepped: the deposit

' can't be juaped over and contact is noraal.

Endif

Until Stuck

'Repeats dancing with this dancer until it's stuck (deposited or killed).

Exit If $210 r$ der $z(1)+5>200$

"Exits the aain loop if grouth is big enough,if the killing circle reaches the edge of the screen. Loop

Gosub Cleanup

- Cleans up the ess before finishing the progras.

End

- Procedure Libraryi

Procedure Nendancer

- Makes a new particle to deposit.

$x y=$ Fianda $(720)$

- Radial location in half degrees, 0 to 719.

$r y=200+$ Int $((\operatorname{Order} z(1)+5)+\operatorname{Cos}(x / \mathrm{tP} i / 360))$

- Generating circle is Reax +5 , $50 y=R C 0 s$ (theta) and

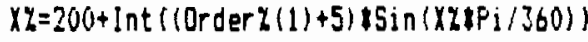

- thet $=($ hal fdegrees $\times \mathrm{pi}) / 360$.

Plot $X \%, Y \%$

'Puts the new dancer on the screen.

Return

Procedure Up 
Sub Yh, Juap\%

- Jup up, so y coordinate is decreaented by the distance to juap.

If $r \&<0$ Then

- If juep is off the screen, wraps around to the other edge,

- (never satisfied with killing circle present; dancer dies first).

Add $Y \%, 400$

Endif

Return

Procedure Down

Add Y\%, Junp\%

'Likewise, only juep is downward (increasing y coordinatel.

If Y\% 399 Then

Sub $Y \%, 400$

Endif

Return

Procedure Left

Sub $x y$, Jusey

'As above, only decrease $x$.

If $x y<0$ Then

Add $x \%, 400$

Endif

Return

Procedure Right

Add $x \%$ Juep\%

If $x y>399$ Then

Sub $x \%, 400$

Endif

Return

Procedure Check

' Checks to see if deposition conditions are satisfied. If they are then, stick, Stuck=True.

If Mot -Point (X\%, Yy) Then

' If the point jueped to is already occupied, then collision is detected

Stuck $=$ True

' and stick at prevoius coordinates ( $\left.X_{0}\right) \mathrm{d} \%$, Yold\%).

Inc Order"

- Records the number of deposits as being one greater.

Order $y(\operatorname{Order} y(0))=x_{0}$ old $z 11024+y 01 d y$

- Encodes and saves the coordinates of the deposited particle.

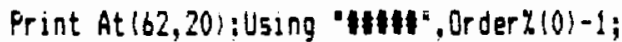

- Displays the oosition

Raz $=5$ ar $\left.\left(\left(x_{0}\right) d y-200\right)^{\wedge} 2+\left(Y_{0} d \alpha y-200\right)^{\wedge} 2\right)$

Print At $(55,24)$; Using 'At)', Ray;

' and the radius of the deposit. Then calculates the angle from the center.

Angley=Atn ( Y Yol dy-200)/(xold $x-200+0.01) / 157.3$

Thet ay=Angl e\%

- This calculates the true angle fron the arctan function, which gives

' angles from -90 to +90 degrees, instead of 0 to 359 degrees.

If Angley<0 Then

Thet a $=360+$ Angl ey

Endif

If Xold 6200 then

Thet ay $=180+$ Angl e\% 
Endif

If Raysorderz(1) Then

- If this is a saxinus radius deposit, then

Order $z(1)=$ Ray

'updates Reaz and

Hangl $\mathrm{e}=$ =Thet $\mathrm{a} \%$

'reports the angle of the saxiaun radius of the deposit.

Enjif

"Prints it all out...

Print At $(75,21)$; Using 'Aft', Order $2(1)$;

Print At $(77,22)$; Lusing 'AH", Manole\%"

Print At $(63,24)$ : Using 'At', Thetah;

Print At $(69,25)$; Using 'At4t:, Hjumps;

' Hakes a been to indicate deposition.

Sound $1,15,1,8,1$

Sound $\$, 0$

Njunp $5 \%=0$

'Resets Njuaps for the new dancer which will be generated. It's here

' 50 Kjuassh is only reset between deposits, not when a dancer is killed

' and replaced: if it were in newdancer, it would count juops only for that dancer.

Endif

Return

Procedure Breakhandler

' If Control-Shift-Reset is key-stroked, comes here and clean up.

Gosub Cleanup

- Does the elean up routine,

On Break

' resets basic language's default Break handler,

End

' and ends the progras.

Return

Procedure Errorhandler

'If an error happens, cones here.

bosub Cleanuo

- Cleans up the aess,

Err $\$=$ Error "tStr\$(Err)+" occurred. | Data duaped to oisk."

' oakes a uessage telling what happened,

Alert 1, Err\$, 1, "Return', $x$ \%

'and displays it. Then...

On Error

' resets error handler to basic's regular one,

End

' and ends the progran.

Return

Procedure Cleanup

- This does the actual work of cleaning up.

If Point (xoldy, Yoldy) $=0$ Then

'If there's a dancer on the screen at an old cocrdinate

Plot Xoldy, Yoldy

'erases it so that it doesn't appear in the SCR file.

Endif

If Point $(x y, y z)=0$ Then 
'Likewise if it's at the new coordinates.

Plat XY..YY

Endif

- Binary saves the screen cantents to the save filenase,

Bsave 8s. X6ios (2).32000

' binary saves the Order array to a file with an ARe extensich.

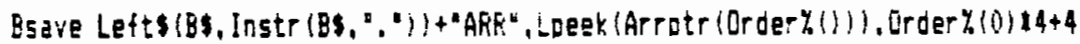

' and announces the saving.

Text 80.64 . Data saved to file" +8 s

Return

In order to display the whole aggregate on the screen at once, it was necessary to limit the maximum size of the aggregate to 30,000 deposits. If a partitioned display had been utilized, the constraints would have been upon the limitations of the computer memory and the amount of time available to run the simulation. The average time to grow the small aggregates was approximately $\theta$ hours and it took 30 hours to grow the large aggregates. If time had not been a factor, then the memory requirements of the Boolean array simulation space and the integer array deposit space, would have allowed for a maximum of approximately 75, 000 deposits. For the large version of the simulation program, the simulation was moved from the screen buffer into the main memory. Additionally the deposit array was a changed from a real number array with nine bit packed words consisting of; the $x$ and $y$ coordinates and the number of "jumps' taken from a pixel's 'birth', to its deposition, into an integer array with ten bit packed words consisting of; the $x$ and $y$ coordinates of each deposit. (The encoding of the of the coordinates saved memory space, allowing the simulation spaces to be 1 arger. In order to have the coordinates of 
the large simulation space to be greater than 512 the coordinates required ten bits.) Although, the simulation space needed four times as much memory as the deposit space, in order to allow for the diffusion zone enclosed in the 'killing' circle, the deposit space could be larger than the memory locations of the deposit array because the deposition was fractal and not compact. Integer arrays require 4 bytes of memory for each element, floating point arrays 6 bytes, and Boolean arrays need only 1 bit for each element. In order to more quickly execute the simulation, deposition was determined by checking the spatial array of the simulation space, rather than the sequential deposit array and then only when the stepsize was a unit step. In the large simulation, the information concerning the 'dancer' or random walker was deleted; the "dancer" or random walker was not plotted, the number of 'jumps' was not counted, and its polar coordinates at deposition were not calculated. Implementation of a smaller 'killing' circle rather than Meakin's, (2R $\max$. vs. $\left.3 R_{\max }\right)$, reduced the time a pixel would be in the diffusion zone, this effectively increased the rate of deposition. (The agreement of the fractal dimension supports this modification. Further analysis was not conducted to investigate whether this simulation was, in fact, less diffusive than Meakin's.) Various look-up tables were used to decrease the run time. Examples are the jump table which gave the lengths of the 
jumps that the random walker took when in the diffusion zone (instead of using the exponential function), and the Pythagorean array which gave radial distances (rather than taking the square root).

Among the programs developed for this research, the more salient are presented below. They are menu driven and are provided with "Help' screens. The Correlate Program calculates the correlation function using exact circles and squares. It is representative and the most developed of the three correlation programs. It provides additional data such as the number of excluded pixels in the edge and the run time, (approximately 24 hours). (The number of excluded pixels was computed with the intention of additional analysis; to determine the connection between the aggregate's geometry, the correlation function results, and the number of excluded pixels.) The look-up table of partial areas is given for only one octant and by employing symmetry, is used for the whole circle.

The Radius of Gyration Program utilizes a running average as it evaluates the deposit array. It also includes the special procedure which corrects for the previously mentioned error and calculates the radius of gyration from the center of mass.

The following programs provide graphical output and analysis; Megamenu is the animation and file maintenance program, Coremenu determines the various mass distributions 
for single aggregates and composites, and the Deposition Frequency Histogram Program also compiles the composites, in addition to. "slicing" the cumulative deposition probability distribution, at any arbitrary deposition probability.

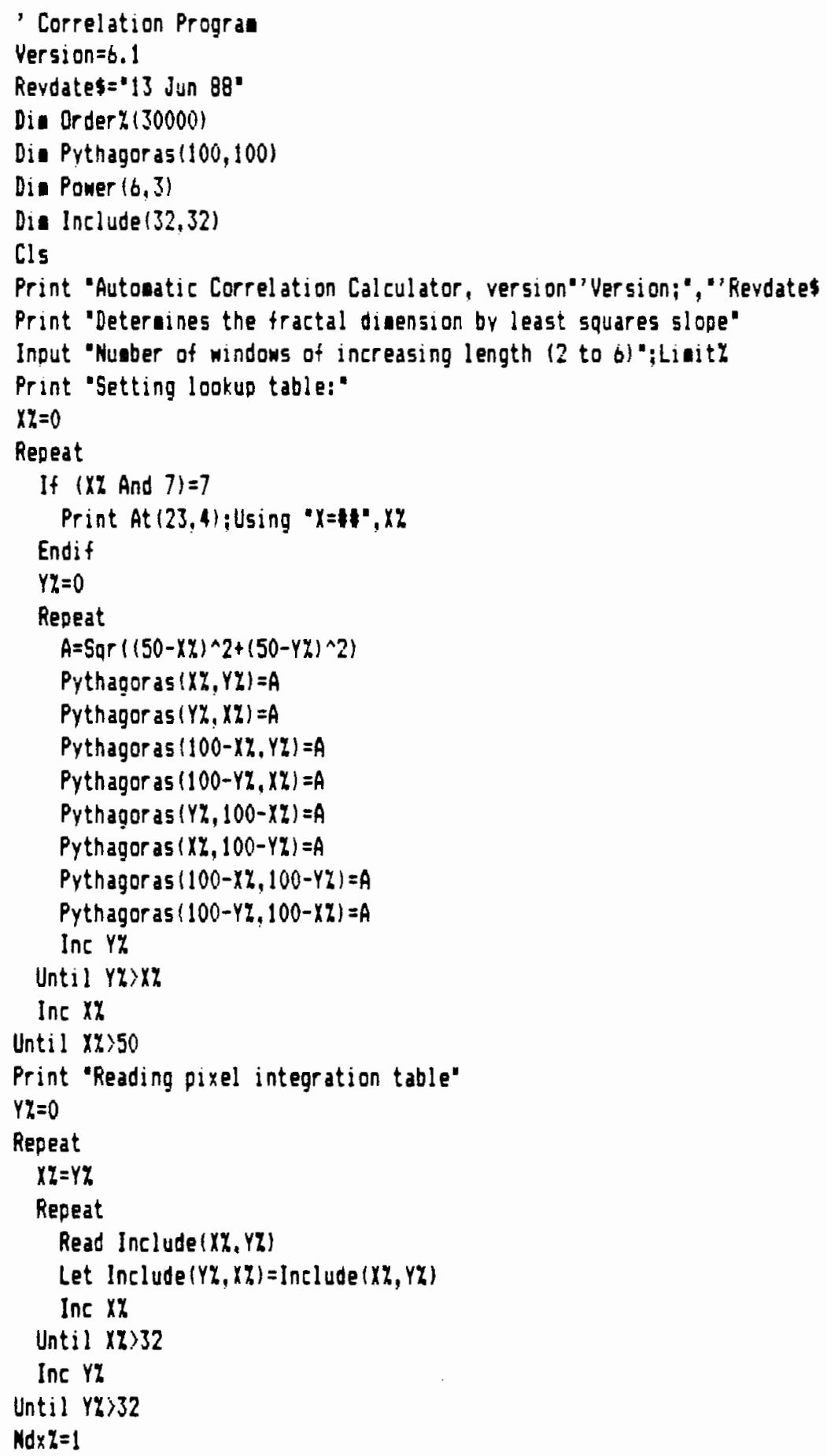


Radius $y=2^{\wedge}(L i n i t z-1)$

Repsat

Power $($ Ndx $\%, 1)=$ Radius $\%+0.5$

Inc Noxy

Div Radius\%, 2

Until Noxh>Lieity

Do

Cls

Showe

Print "Choose Mode of Operation: Type nuber or elick on selection."

Print

Print " 1 Autonatic processing of all . ARR files on disk"

Print

Print "z Use alreadv sreated directory of filenanes (CORELATE. DIF) -

Print

Print "J Process single file"

Print

Print '4 Helpful hints and instructions"

Print

Print "5 Exit"

Graphaode 3

Deffill 1,1

Ptrver to0s\%=housey

If Frac (Ptrvertoos\%/32) 0.5 Then

Gosub Inbou (Ftrvertpos\%)

Else

$\ln i=0$

Endif

Do

Repeat

Ptrvertpos:h=Housey

If (In:>0) And (Frac (Ptrvertpos\%/32)>0.5) Then

Gesub Dutboy (Ptryertoos\%)

Endi:

If $(\operatorname{In} \%=0$ ) And (Frac (Ptrvertpos $\% / 32)<0.5)$ Then

Gosub inbox (Ptryertoos\%)

Endif

jwitchis=Housek

If Switchiso then

If Iny $y 0$ Then

Switchy $=($ ftryertoos $\%(32)-2$

Else

Switeh $\%=0$

Sound $1,15,6,7,5$

Sound 1,0

Endif

Endif

Keys=Inkeys

Until Keys Y" Or Suitchy

If Switchy Then

Key $s=S t$ s (Switchy)

Endif 
Exit If Val (Key $\$ 1>0$ And Val (Key $\$(6$

Sound $1,15,6,7,5$

Sound 1,0

Loop

$\mathrm{Cl} 5$

Graphoode 1

On Val (Kev") Gosub Auto, Existingfile,Single, Help, Exit

$\ln \%=0$

Switeh $y=0$

Loop

End

Procedure Inbox (Htw)

$H t y=32 t(H t x) 32)$

If $H t \%>16$ And $H t \%<192$ Then

Pbox $-1, \mathrm{Ht} \%, 500, \mathrm{Ht} \%+16$

In\%=Ptrvertpos\% 132

Endif

Return

Procedure Qutbon (Ht:

$H+\%=32 \ln \%$

Pbox -1, Ht $\%, 500, H t \%+16$

$\ln \%=0$

Return

Procedure Exit

Edit

Return

Procedure Help

C15

Print " This progra can run in autonatic node. The requirements are that"

Print "it sust be given a disk with a series of .ARR files with their"

Print "associated .SCR files. There can be no other .ARR files on the dist."

Print "If there are no. ARR files in the current disk or directory, a bus"

Print "error (two bonbs) will result."

Print "To use the pre-existing directory code leg. to do only soae of"

Print "the .ARR files on a diskl, create a text file naned CORELATE.DIR,"

Print "containing the filenaes of then .ARR files you wish to process."

Print "Each filenane should appear on a single line in the file."

Print " In both these cases, the results go into a file called CORELATE. DAT"

Print "in a tabular form, with the filenaee at the top, followed by lines"

Print "with three nuabers separated by cousas. These represent $R$, Mdisk(R),"

Print "and Ksquare(f) for each $R$ processed (ndisk is the aver age pixel"

Print "density in a disk of radius R). The slopes of the best-fit power"

Print "curves for each technique are printed on the next two lines. These"

Print "slopes are the fractal diensions as deterained by the two-doint"

Print "correlation function over disks and squares respectively. The total"

Print "nuber of deposits and the number of Dixel excluded to eliainate edge"

Print "effects are printed on the last two lines."

Print "The single file ode allows you to process a single file on the"

Print "disk, which can be entered froe a Fileselect box. The results do not"

Print "go into a file, but are just printed on the screen."

Print - Hit any key to continue"

Repeat 


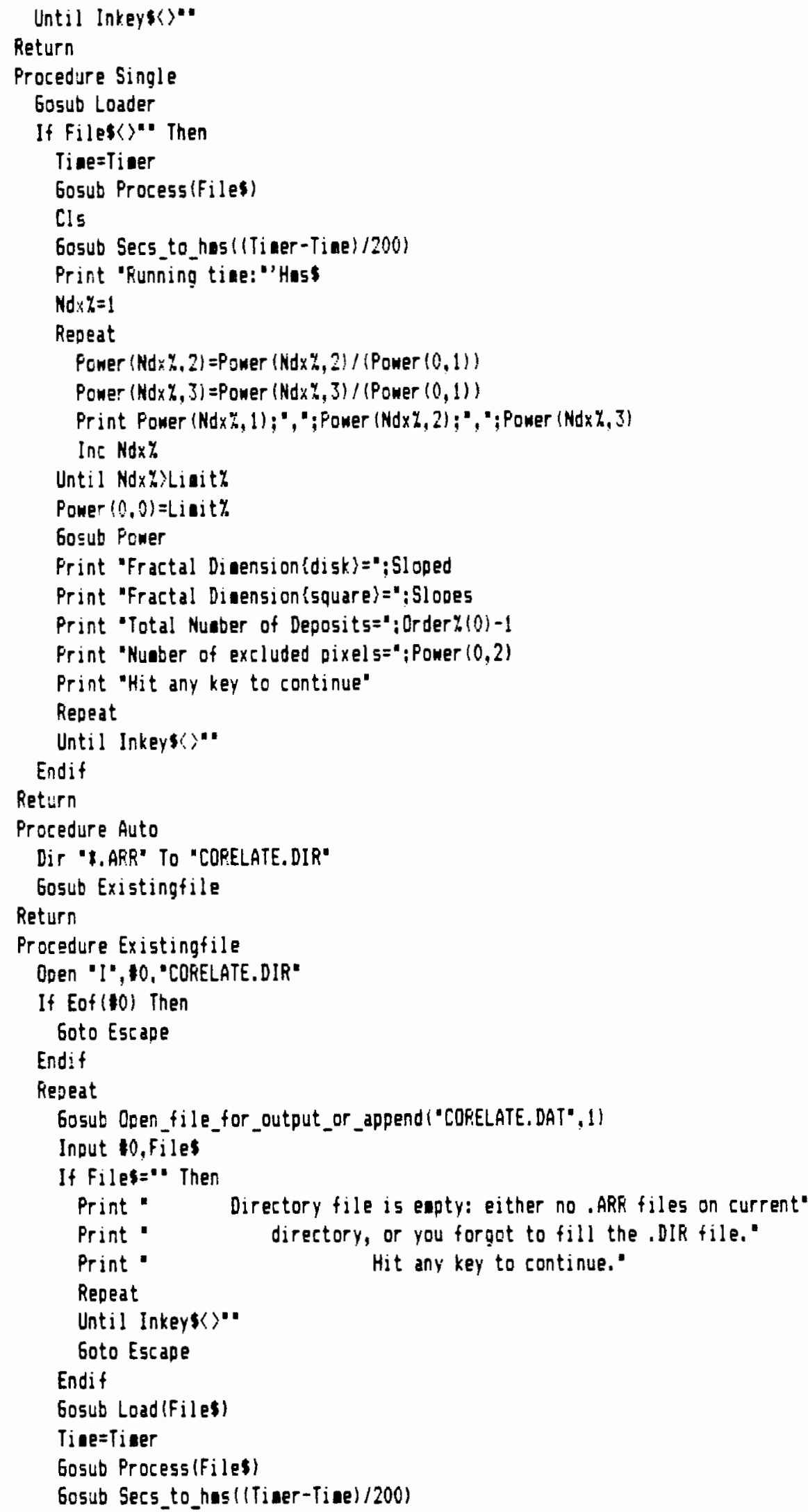


Print 1l, Files

Print 11, "Running tice:" Hass

$\operatorname{No} x \mathrm{z}=1$

Receat

Power (Ndx $\%, 2)=$ Power $(N d x \%, 2) /($ Power $(0,1))$

Poner (Ndxy,3)=Power (Ndx $h, 3) /($ Power $(0,1)$ )

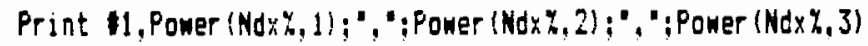

Ine Ndey

Until Noxyliaity

Power $(0.0)=$ Liaity

Gosub Fower

Print 1, "Fractal Diension\{disk\}=', Sl oped

Print 11,"Fractal dieension $\{$ square $=$ ": Slopes

Print 11."Total Nubuer of Deposits=";Ordery(0)-1

Print 11. "Nuaber of excluded oixel $s={ }^{2}$; Power $(0,2)$

Close 11

Inti! Eof $(10)$

Estape:

Close

Return

Frocedure Process (File\$)

Deffill 0,1

Pbo: $401,0,630,390$

Deffill 1,1

Ruindosax $y=$ int $($ Power $(1,1))$

Rdepositnax $h_{h}=$ Order $z(1)$

Nd $x y=1$

Poner $(0,1)=0$

Power $(0,2)=0$

Repeat

Power $(N d x \%, 2)=0$

Power $(N d x \%, 3)=0$

Inc $N d x \%$

Until Ndxylioity

Print At (53,3): 'File: ':'Files

Print At $(53,5):{ }^{\circ} \mathrm{N}=0$ "

Print At(53,7);Using "Out of tftt\$ total deposits', Order\%(0)-1

Print At $(53,9)$ : "Excluded pixels $=0$ "

$N 4=2$

Reseat

$x_{w} y=\operatorname{Order} z(N z) \cup 1024$

$Y_{n} y_{h}=\operatorname{Brder} y_{(N y)}(N)$ And 1023

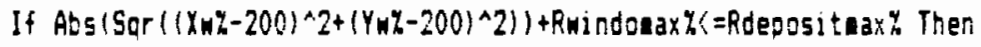

Ine Power $(0,1)$

$x y=x w^{y}$-Riwindonaxy

Repeat

$Y$ Y $=Y_{w}$ h-Rwindosax ?

Repeat

If Point $(x y, y y)$ Then

Roi $x=$ Pythagor as $\left(x z_{m}-x_{m} y+50, y z-y_{w} z+50\right)$

$M d x z=1$

Repeat 


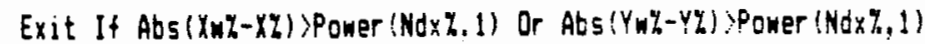

Inc Power (Ndx $y, 3)$

Exit If Roix >Power (Noxt., 1) +0.70710678119

If Roix $<$ Power (Ndx \%, 1)-0.70710678119 Then

Inc Power (Nd:Y, 2)

Else

Corner $=$ Sgn $($ Power $(N d x \%, 1)-$ Foj $x) 10.5$

Renr $=($ Abs (Abs $(X z)-A b s(X w))+$ Corner $)^{\wedge} 2$

Add Ronr, (Abs (Abs (YZ)-Abs (YwY)) +Corner)^z

Renr=Sar (Renr)

If Power (Ndx ${ }^{2}, 11$ ) Min (Roix, Renr) And Fower (Ndx\%,1) (Max (Roix, Renr)

Add Power (Nexy, 2), Include (Abs $\left(X_{w} y_{2}-X_{\%}\right)$, , Abs $\left.\left(Y_{w} w_{0}-Y z\right)\right)$

Else

If Power (Ndx\%, 1) )Fipix Then

Ine Power $($ Non $y, 2)$ Endif

Endif

Ens: $f$

In: Ndxy

Until Nexisulaty

Endit

Ine Yy

Until Yy YYwy+Rwindomaxy

$\ln x \times \%$

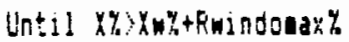

El se

Ine Power $(0,2)$

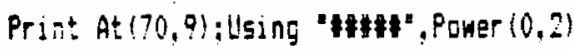

\section{Endif}

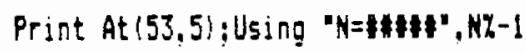

Ine N\%

Option "U1"

Unti! Nh>Order\%(0)

Return

Frocedure Loader

Print At (1.3): "Select array:

Fileselect 'U.ARR'" "SEED. ARR', Files

If Filew" Then

Gosub Load(Files)

Endif

Return

Procedure Load(Files)

Hidea

Arravfill Orderz (1):0

Bload Files, Lpeek (Arpotr (Order z1))

Gosub Parsefilenane(Files)

Bload Pathnanes+" 1 "Left (File\$, Instrifiles, ". "1)+"SCF", Xbios (2)

Return

Procedure Parsefilenane (Fns)

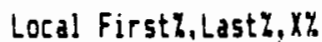

Pathnaes $=$ Left $\$$ (Fns, Instr (Fns, ": '))

Firsty=lnstr $(F n, ")\}$ 
For $x z=\operatorname{Len}(F n)$ Domnto 1

If Mids $\left(F \cap s, X_{n}, 1\right)=-$ !"

Lasty $=x \%$

Endi:

Exit If Mids (Fns, XY, 1) ="

Next $X \%$

Pathnaes=Pathnane\$+hido (Fns. Firsty,Lasty-Firsty)

Files=hids (Fns, Lasty +1$)$

Return

Procedure Ooen_file_for_outout_or_acpend (Filet.Chanly)

It Not Existifilesi then

Open "li", tharil\%, Files

Else

Open "A", thanl\%,Files

Endif

Return

Procedure Power

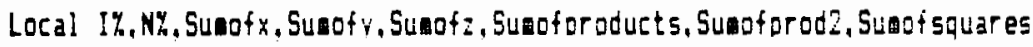

$N_{h}=$ Fower $(0,0)$

Suact $x=0$

Suarof $v=0$

Sunot $i=0$

Suaforoducts $=0$

Sugotprod $2=0$

Sunof souares $=0$

For $1: i=1$ To $\mathrm{NY}$

Add Sunof $x$, Log (Fomer $(I \%, 1))$

Add Sunofy, Log (Fower $(I \%, 2\})$

Add Sunof $z$, Log (Fomer (I: 3,3$)$ )

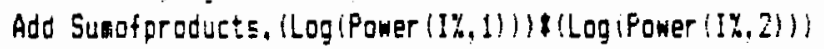

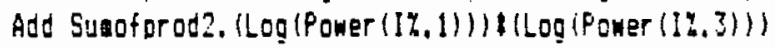

Add Suncisquares, $(\log ($ Fower $11 \%, 1)))^{\wedge}$ ?

Next 1\%.

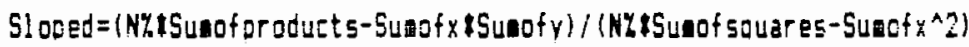

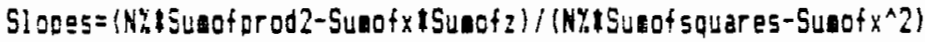

Return

Procedure Secs_to_has (Secs)

Local $H, H, S$

Has $="$ "

$H=5 e c 513600$

$M=($ Secs Mod 3600$) 160$

$S=($ Secs Mad $3600 ! \operatorname{Mod} 60$

If $H>0$ Then

Has $\$=$ Str $\$(H)+{ }^{\prime}$ hours,

Endif

Hes $\$=$ Hass+Str $\$(M)+$ " inutes, "+Strs(S)+" seconds"

Keturn

Data $1, .97173982736, .98323197634,1, .99072351790,0,0,0, .99509549182$

Data $0,0,0,0,0,0,1, .99747439951,0,0,0,0,0,0,0,0,0,0,0,0,0,0,1 . .99971790316$

Data .54540604028, .76932502669,1,.87746746419,0,0,1,.93596316355

Data $0,0,0,0,0,0,1, .96712950449,0,0,0,0,0,0,0,0,0,0,0,0,0,0,1, .9833279216$

Data . $13685659153,1, .51818108335,0,0,1, .75601286272$ 
Data $0,0,0,0,0,0,1, .87575702090,0,0,0,0,0,0,0,0,0,0,0,0,0,0,1, .93711375142$

Data $.79041291337, .040939641236,0,0,1, .44699616090$

Data $0,0,0,0,0,0,1, .72232444282,0,0,0,0,0,0,0,0,0,0,0,0,0,0,1, .859943588826$

Data $0,0,1, .92966414755, .063188476255,0,0,0,0,0,0,1, .50504463758$

Data $0,0,0,0,0,0,0,0,0,0,0,0,0,0,1, .75159505196$

Data $1, .99978095027, .36479676534,0,0,0,0,0,0,0,1, .22126674792$

Data $0,0,0,0,0,0,0,0,0,0,0,0,0,0,1,61175243929$

Datà . $50713675836,0,0,0,0,0,0,0,1, .86285867404, .00444268875975$

Data $0,0,0,0,0,0,0,0,0,0,0,0,0,0,1, .44000194594$

Data $0,0,0,0,0,0,0,1, .43815088971,0,0,0,0,0,0,0,0,0,0,0,0,0,0,0,1$

Data. 23582534771

Data $0,0,0,0,0,1, .88988358922, .037209702477,0,0,0,0,0,0,0,0,0,0,0,0,0,0$

Data $1 . .96754775755$. . 031044089235

Data $0,0,0,1, .99993904191, .32508603414,0,0,0,0,0,0,0,0,0,0,0,0,0,0,0,1$

Data . 72754780948,0

Data $0,0,1, .61938262358,0,0,0,0,0,0,0,0,0,0,0,0,0,0,0,0,1$

Data $.42180377617,0$

Data $1, .76123760181,031030323298,0,0,0,0,0,0,0,0,0,0,0,0,0,0,0,1$

Data $.98595157766 .0 .09436695: 496,0$

Data . $05568853789,0,0,0,0,0,0,0,0,0,0,0,0,0,0,0,0,1, .70187921077,0,0$

Data $0,0,0,0,0,0,0,0,0,0,0,0,0,0,0,0,1,28507660143,0,0$

Data $0,0,0,0,0,0,0,0,0,0,0,0,0,0,1, .82387863618, .0043956641457,0,0$

Data $0,0,0,0,0,0,0,0,0,0,0,0,0,1, .32956993207,0,0,0$

Data $0,0,0,0,0,0,0,0,0,0,0,1, .782780131, .0039658586902,0,0,0$

Data $0,0,0,0,0,0,0,0,0,1, .989801506335, .20740175794,0,0,0,0$

lata $0,0,0,0,0,0,0,0,1, .55789679661,0,0,0,0,0$

Data $0,0,0,0,0,0,1, .83028649833,0344906049143,0,0,0,0,0$

Data $0,0,0,0,1, .95098614268, .16377227806,0,0,0,0,0,0$

Data $0.0 .1, .99069313362, .31065568299,0,0,0,0,0,0,0$

Data $1, .99880149052,41972986399,0,0,0,0,0,0,0,0$

Data $.45948678866,0,0,0,0,0,0,0,0,0$

Data $0,0,0,0,0,0,0,0,0$

Data $0,0,0,0,0,0,0,0$

Data $0,0,0,0,0,0,0$

Data $0,0,0,0,0,0$

Data $0,0,0,0,0$

Data $0,0,0,0$

Data $0,0,0$

Data 0,0

Data 0

- Radius of Eyration Progras

Version $=1.7$

Revdate $\$=29$ Det $88^{\prime}$

Dis Order $\%(30000)$

Di. Radii $(1,400)$

Dis Pythagoras $(100.100)$

Do

$\mathrm{Cl} 5$

Showe

Print "Autonatic Radius of Gyration Calculator, version" Version:","Revdates

Print "Choose Mode of Operation: Type nuaber or elick on selection." 
Print "I Autonatic processing of all . ARR files on disk"

Print

Print " 2 Use already created directory of filenanes (GYRATE.DIR)"

Print

Print " 3 Process single file"

Frint

Print "4 Heloful hints and instructions"

Print

Print '5 Exit"

Print "b Special processing of single file"

Graphoode 3

Deffill 1.1

Ptrver toos $\%=$ Housey

If Frac (Ptrvertoos" $/ 321<0.5$ Then

Gosub Inbo: (Ptrvertpos\%)

Else

$\ln \frac{y}{2}=0$

Endit

Do

Repeat

Ptrvertpos\%=Housey

If $(I n \%) 0)$ And (Frac (Ptrvertoos\%/32)>0.5) Then Gosub Dutbox (Ptrvertoosh)

Endif

If (In\%=0) And (Frac (Ftrvertpos\%/32)<0.5) Then Gosub Inbox (Ptrvertoos\%)

Endif

Switchy=Housek

If Suitchysy Then

If $\ln y) 0$ then

Switch\%=(Ftrvertoos\%(32)

Else

Suites $\%=0$

Sound $1,15,6,7,5$

Sound 1,0

Endif

Endi!

Key $\$=$ Inkeys

Until Keys(>" Or Switch"

If Suitchl Then

Key $=$ Strs $($ Switchy)

Endif

Exat If Val (Keys) >0 And Val (Key $\$ 17$

Sound $1,15,6,7,5$

Sound 1,0

Loop

$\mathrm{Cls}$

Graphoode 1

On Yal (Key\$) Gosub Auto, Existingfile, Single, Helo, Exit, Special

In $y=0$

Suiten $y=0$ 


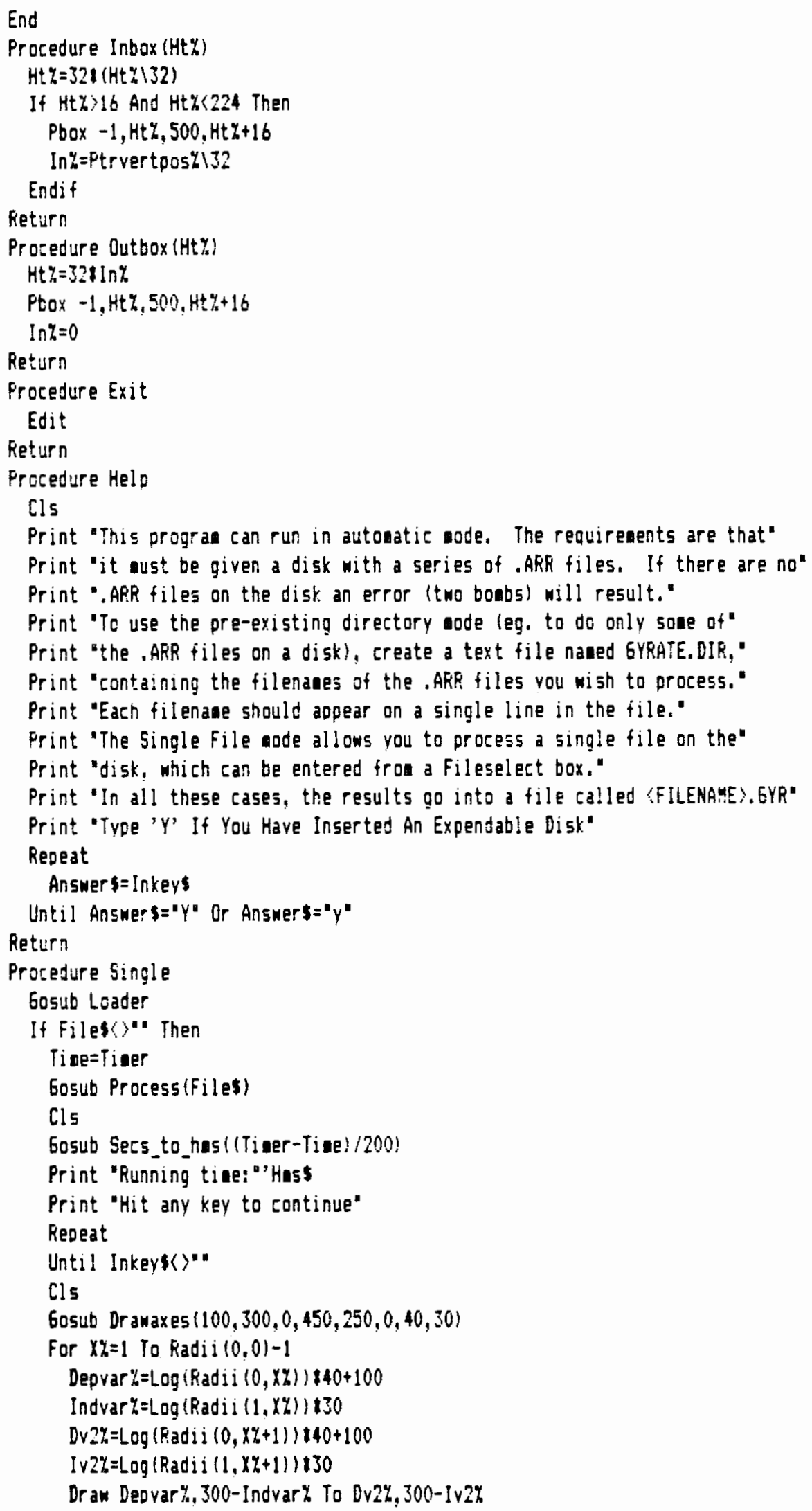




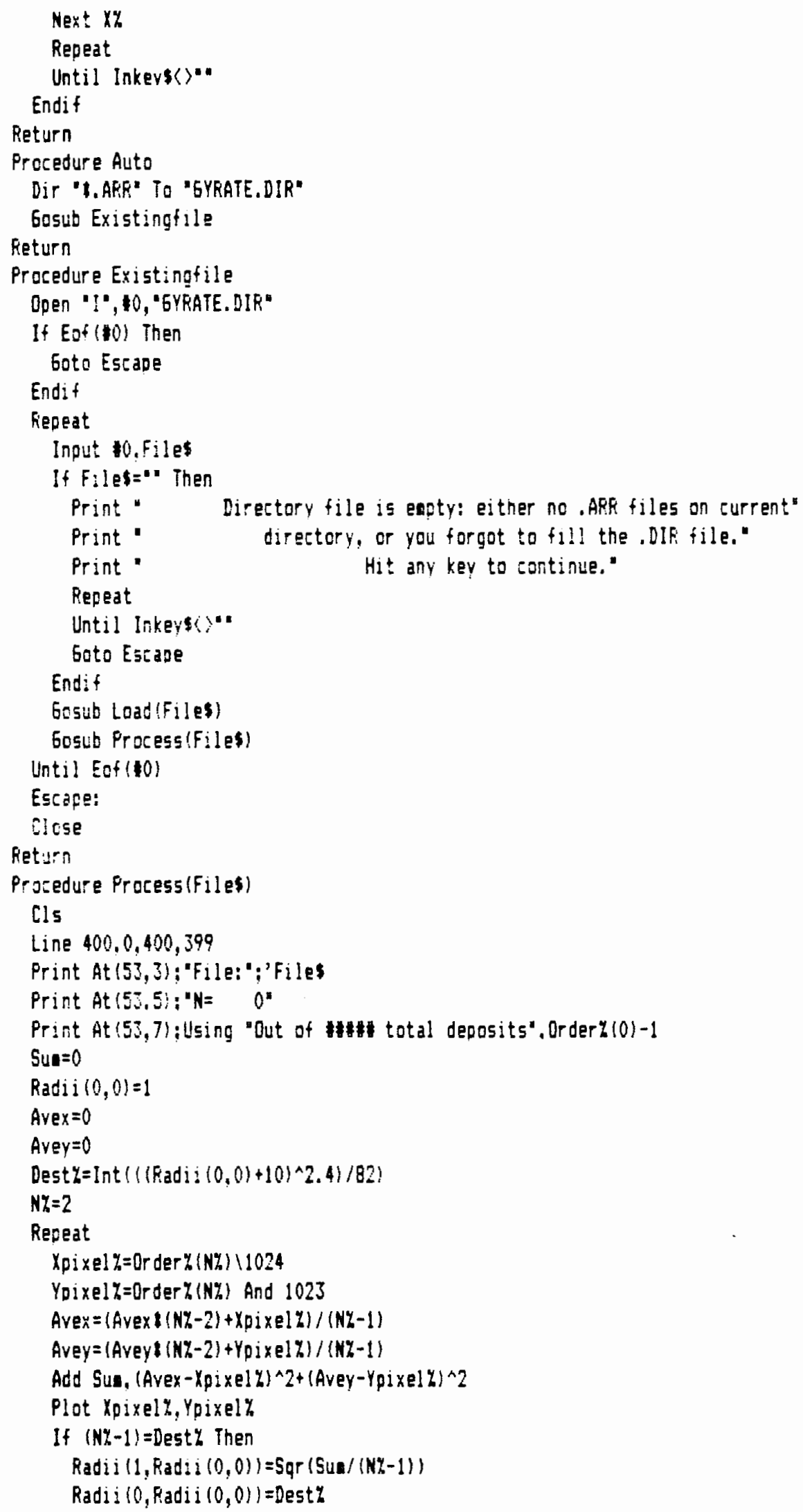


Inc Radii $(0,0)$

Dest $y=\operatorname{Int}((\operatorname{Radi} ;(0,0)+(0) \cdot 2.4) / 82)$

Endif

If $\left(N_{h}^{2}-1\right)$ Mod $100=0$ Then

Print At $(53,5)$; Using 'N=\$AtA4', $\mathrm{Nz}-1$

Endi?

Ine $\mathrm{N} \%$

Dption 'U1"

Until Ny>Order\%(0)

Dec Radii 10,0$)$

Gosub Parsefilenaae (Files)

Files =Pathnane\$+" $\mid$ "Left iFiles, InstriFiles, ". "I)+"EYA"

Bsave Files, Loekk (Arrotr (Radij(1)), (Radii $(0,0)+1)(1 ! 2+8$

Return

Procedure Loader

Print At $(1,3)$ : "Select arrav:

Fileselect "U. ARP" "SEED.ARR", Files

If Files, "Then

bosut Load(Files)

Endif

Return

Procedure Load(Files)

Hiden

Arravfill Order\%(1),0

Arraytill Radii(),0

Bload Files, Lpeek (Arrptr (Order:111)

Return

Procedure Farsefilenane (Fns)

Local First\%,Lastz, X\%

Pathnames=Left (Fns, Instr (Fons, ": ")

Firsty =instr (Fn\&, $\cdots)$

For $x y=\operatorname{Len}\left(F_{n}\right)$ Downto 1

If $\operatorname{Mids}\left(F n s, x_{y}, 1\right)=\cdot 1$

Lasty $=X Y$

Endif

Exit If Mids(Fns, $x h, 1)=\cdot$ ?"

Next $x \%$

Pathnate = Pathnames+Hids (Fns,Firsty,Lasty-Firsty)

Files=Hids $(F n s, L a s t \%+1)$

Return

Procedure Pomer

Loca! 1\%, N\%, Sunof $x$, Sunofy, Sunof $z$, Suchefproducts, Sunofprod2, Sunof squares

$N y=\operatorname{Power}(0,0)$

Sun of $x=0$

Sunafy $=0$

Sunof $z=0$

Sunof products $=0$

Susofprod $2=0$

Sunof squares $=0$

For II $=1$ To $N y$

Add Suaof $x, \log ($ Power $(1 \%, 1))$

Add Sula of $v, \log$ (Fower $(I \%, 2))$ 
Add Sunof $z$, Log (Pomer!1\%,3))

Add Sunofproducts, (Log (Fomer $(1 \%, 11)) 11$ Log (Pawer $(1 \%, 2))$ )

Add Sulof prod2, (Log(Fower $(1 \%, 1)$ ) 1 (Log (Pawer $(1 \%, 3))$ )

Add Sunof sauares, ILog (Power $(1 \mathrm{~h}, 1 \mathrm{H}))^{\wedge} 2$

Next Iy

S1 oped $=($ N\%tSunof products - Sunof $x$ \&Sunof $y) /\left(\right.$ N\%t tSunof squar es - Sunof $\left.x^{\wedge} 2\right)$

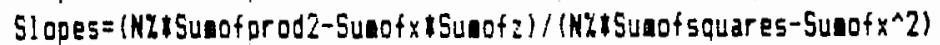

Return

Procedure Secs_to has (Secs)

Loral H, M, S

Hes $\$=" 4$

$H=5 e \operatorname{sis}: 3600$

$h=15$ ecs Mod 36001160

$S=($ Secs Mod 3600$)$ Mod 60

If $H>0$ Then

Hess=Str $\$(H)+"$ hours, "

Endif

Has $s=$ Has $\$+S t r \$(H)+2$ minutes, "Str $\$(5)+"$ seconds"

Return

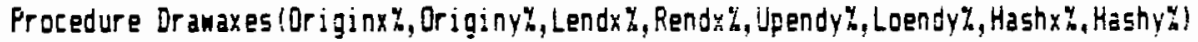

Defline $1,1,1,1$

If Lend $x=$ ? Then

Defline $1,1,0,1$

Endif

If Rend $y_{0}=0$ Then

Def! ine $1,1,1,0$

Endif

Dran Originxy-Lendx\%, Originy" To Originx"+Rendx

Defline $1,1,1,1$

If Upendy $y=0$ Then

Defline $1,1,0,1$

Endif

If Loendy $h=0$ Then

Defline $1,1,1,0$

Endif

Draw Originx\%, Originy"-Upendy\% To Originxz,Originyz+Loendv\%

Local Ay, Length\%

Lengthy $=10$

Defline $1,1,0,0$

If Hashx\%:>0 Then

For $A$ z =Originx\% To Originay-Lendxy Sted -Hashx\%

Draw Ah. Originvz-Lengthz To Ah, Originvy+Lengthy

Next A\%

For Ay=Originxy To Originxh+Rendxy Step Hashxy

Draw Ah, Originyb-Lengthz To Az, Originyz+Length\%

Next $A$ \%

Endif

If Hashy: $>>0$ Then

For Az=Originyz To Originyz-Upendy\% Step -Hashy"

Draw Originxz+Lengthz, AZ To Originxz-Lengthy,A\%.

Next Ay

For $A$ y=Originy" To Originyz+Loendyz Step Hashyz 
Draw Originxz+Length\%, A\% To Originxh-Length\%, A\%

Nent $A \%$

Endif

Return

Procedure Centerofoass (P. array, Loty)

$N K=0$

Swap IP. array, Ayearrayz(1)

Avex $=0$

Arey $=0$

Do

Inc $N \%$

Exit If Ny)Laty

Avex $=($ Avex $((N)-1)+($ Avearrayz $(N y+1)) 1024)) / N z$

Avey $=($ Avey $($ (N\%-1) $+($ Avearray $h(N \%+1)$ And 1023$)) / N \%$

Loop

Swap IP. array, Avearraył()

Return

Procedure Special

Gosub Loader

If Files<'" Then

Do

Print "Input nuaber of deposits to include in Rg (up to "iOrder\%(0)-1:", 0 to quit) ":

Input Linity.

Exit If Linit $=0$

Gosub Centerof ass (torderz(), Lisity)

Print "Center of ass =" Avex-200:" "200-Avey

Print "Distance Center of Mass to Origin $="$ 'Sqr $\left(\left(\right.\right.$ Avex-200)^ $\left.2+(\text { Aver-200 })^{\wedge} 2\right)$

Gosub Specialprocess (File\$)

Print "Ln(t of deposits) =" $\log (L i$ it $(\%)$

Print $\operatorname{Ln}(R g)=$ ' $\log ($ Sqr (Sus/(Linith)))

\section{Loop}

Endif

Return

Procedure Specialprocess(filet)

Sun $=0$

$N y=2$

Repeat

Xpixei $y=\operatorname{Order} y(N Y) \backslash 1024$

Ypixely=0rder (Ny) And 1023

Add Sus, (Ayex-Xpixely)^2+(Avey-Ypixelz)^z

Inc N\%.

Option "UI"

Until Wh>lioity

Return

- Meganenu Progras

Version $=4.3$

Reviates=' 29 Jun 88"

Dis Order (30000)

Dis Order\%(30000)

Dis Menus $(50)$

Let Menus $(0)=$ 'Desk" 
Let Menus $(1)==^{-}$Utilities info"

Let Menus $(2)=1-$

For $1=3$ To 9

Let Menu $\$(I)=5 \operatorname{st} \$(I)$

Next I

Do

Ine I

Read Menus (I)

Exit If Menus $(I)=0+4$

LOOp

Data "Exit"." Quit "" "Utilities", Invert"," Display SCR file

Data " Dunp to printer"." Strio data lines". Vlew array file","

Data "Anination"." Load ARR file ","-...-...-.-.-.-.-." Aninate"

Data " Involute"," Zonal grouth",",","

Henu Menus ()

On Menu Gosub Handle_it_for_oe

Print At $(1,3)$; "Menu Progran Version"'Version:", "Revdate\$

Do

On Menu

Loop

End

Prosedure Handle_it_for_me

Cls

If Henu $(0)=1$ Then

Gosub Give_info

Else

On Henu(0)-11 Gosub Quit, Duany, Duany, Invert, Di sp, Prscreen, Strio, Viewarr

If Menu(0) $>19$ Then

On Menu (01-19 Gosub Dunsy, Duncy, Loader, Dunay, Ani eate, Involute, Zona! Endif

Endif

Menu Menus ()

Print At $(1,3)$; "Select function:

Return

Pracedure 6ive_info

Return

Procedure Quit

Menu kill

Edit

Return

Procedure Invert

Print At $(1,3)$ : "File to invert: "

filesel ect "It.SCK", "SEED. SCK", As

If As $>$ " Then

Hides

Blodd As, Xbios(2)

For $x y=x$ bios (2) To $x$ bios (2)+31998 Step 2

Dpoke Xy, Not Dpeek (xy)

Next $x y$

Bsave As, Xbios (2), 32000

Showe

Endif 


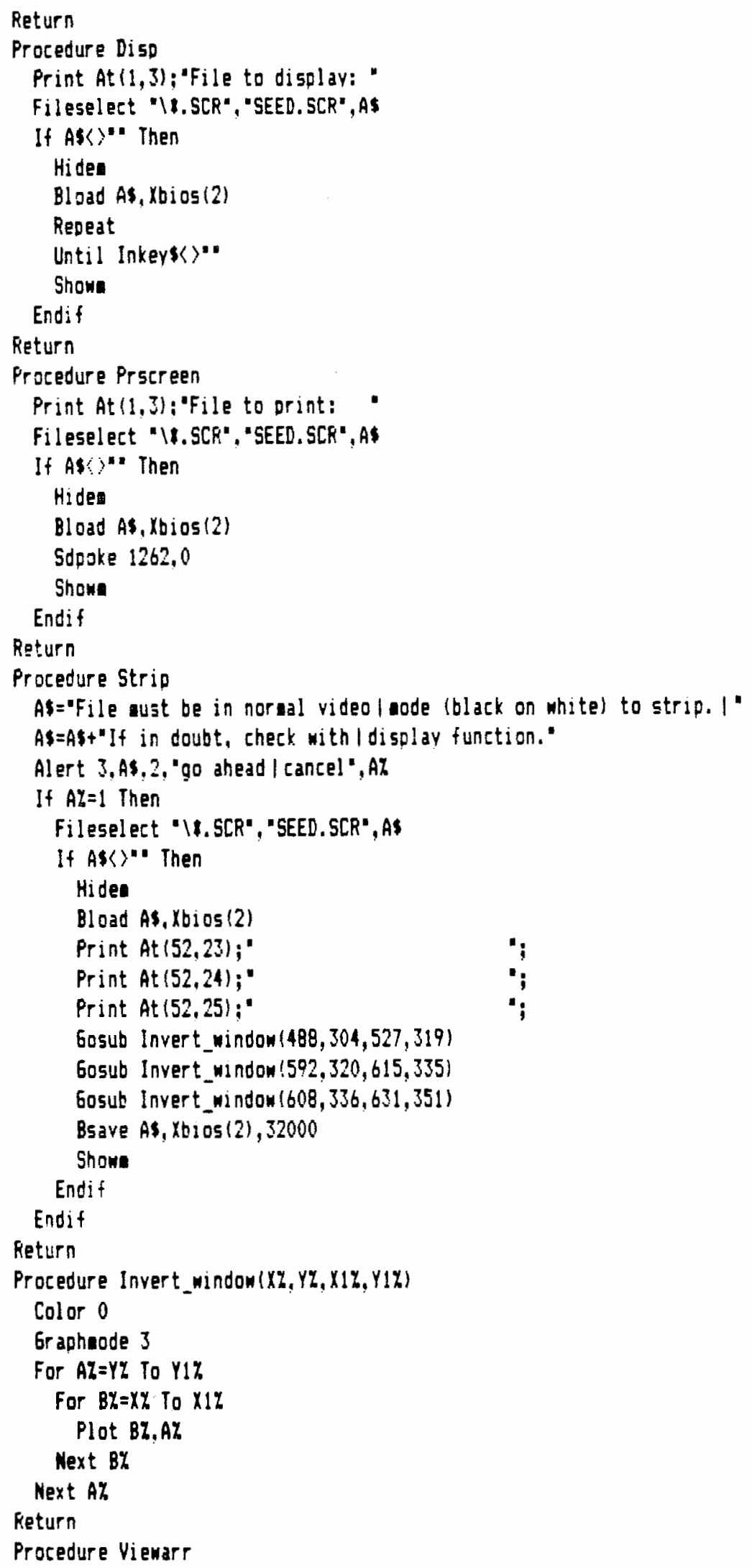




\section{Begin:}

Local Start $\%$, Line $\%$,Len $\%, A s, 01 d !$,Optr $\%$, Notr $\%$, Changed!

Changed! =False

Optry =Lpeek (Arrptr (Order ())

Notr $h=L$ Leek (Arrptr (Orderq(1))

Lpoke Optr\%, 30001

Looke Nptrin, 30001

Arrayfill Order 11,0

Arrayfill Order $\%(1), 0$

Print At $(1,3)$; "Array file to view:";

Fileselest "It.AR?", "SEED. ARR", Arr"s

If Arrs)" Then

Bload Arrs, Optry

Old! = True

Leny=Order (0)

If Order (1) C)Int(Order(1)) Then

Baove Optr\%, Nptr\%,8

If Order $y(0)<30001$ Then

Buove Optr \%, Nptr\%, Order $\%(0) 14+8$

Else

Beove Optr\%, Nptr\%, 120008

Endif

Old! =Fal se

Endif

If Not 0ld! Then

Len $y=0$ order $y(0)$

Endif

If Iristr ('23456789', Rights (Arr\$, 1) ) $=0$ Then

Gleny=Len\%

Seg $\%=1$

Seguentz $=0$

El se

Open ' $R$ ', $\$ 1$, Lef t (Arrs, Len (Arr $\$)-1)+{ }^{a} R^{\prime}, 4$

Field 1,4 As Buf

Get 11,2

6lenh=Cvl (Bufs)

Close 1

Segl=Val (Right $)$ (Arrs,1))

Segaent $y=29999($ Seg\% -1$)$

Endif

Gosub Vi ewarrscreen

Do

For Linez=5tarty To Start $y+23$

If 01d! Then

If Liney $=0$ Then

Print At $(1,2) ;$

Endif

$N="$ Order (Line $\%)-1 ; "$

If Linel $=1$ Then

Print At $(1,3) ; "$

Endif

If Linely And Line\%<3000! Then

Print At (1,Linez-Start $\%+2)$; Using "illt "Line $\%-1$; 


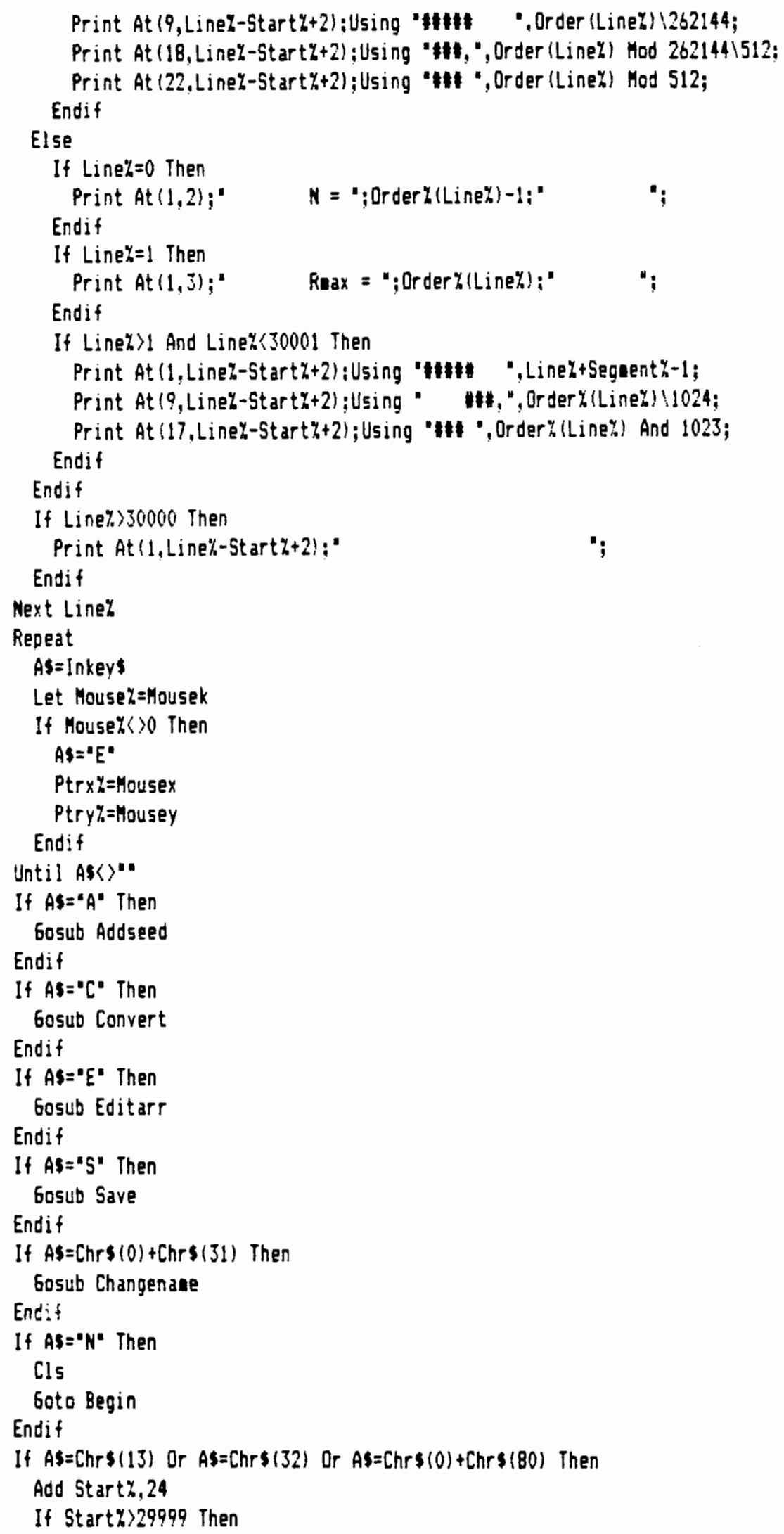




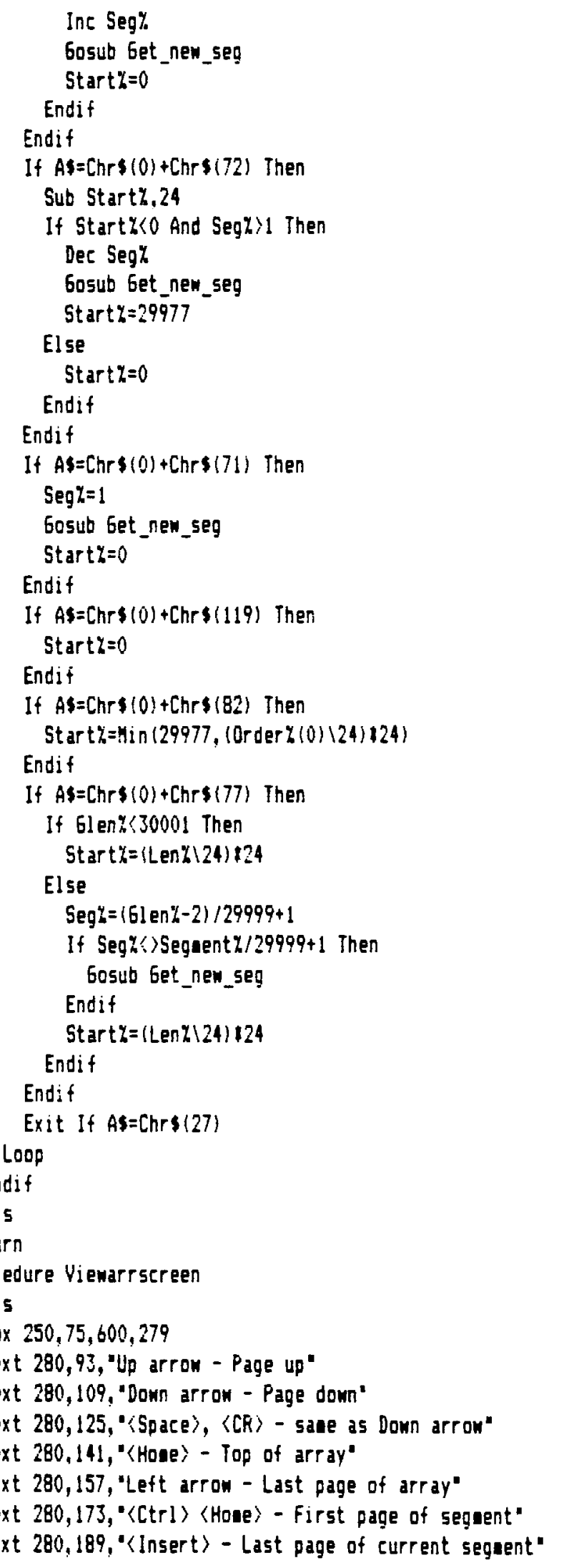


Text 280,205," "ZEsc $\rangle$ - Main menu"

Text 280,221, 'iShift) $C$ - Convert file'

Text $280,237, "\langle$ Shift $\rangle$ A - Add seed Doint to file"

Text 280,253 " "Shift) 5 - Save oodified file"

Text $280,269 . "\langle A l t\rangle S$ - Change filenaae and save"

Start $y=0$

If 01d! Then

Print At $(4,1)$; "N";

Print At $(9,1) ;$ "Juaps";

Print At $11:, 11 ;{ }^{2} x^{2}$;

Print At 23,11$)^{\mathrm{y} Y}$;

Print At $(54,1)$ ' $^{01 d}$ strle array"

Else

Print At 14,1$)$ : 'N';

Print At 114,1$) ; " x "$;

Print At $(18,1)$; "Y";

Print At (54,1): "Nen style array"

Endif

Print At 132,11 : Arr

If Changed! Then

Print At $(54,2)$;'t File Changed!!!"

Endif

Return

Procedure Get_new_seg

If Changed! Then

Print At $(52,20)$; 'Writing changed seguent..."

Gosub Save

Endif

If Seg $\%=1$ Then

Arr $\$=$ Left $($ Arr $\$$, Len (Arr $\$)-1)+' R$ '

Else

Arr $\$=$ Left $($ Arr $\$$, Len (Arr $)-1)+5 t r($ Seg $Y)$

Endif

Arrayfill Order $\%(1,0$

Seguent $y=29999($ Seg $\%-1)$

Print At $(32,3)$; "Loading segrent"'Seg\%; "... Please wait."

Bload Arr\$, Notry

Len $\%=$ Order $\%(0)$

Gosub Viewarrscreen

Return

Procedure Addseed

Local Seedlocation, A\%

Seed! oc at i on $=205000$

If Old! Then

$A y=$ optry

Else

$A y=$ Notry

Endif

Cls

Print "l's checking the length block of "; Arr"'"=':Leek (Ay)

If Loeek (AZ) >30001 Then

Print 'l' resetting the length block to 30001 " 


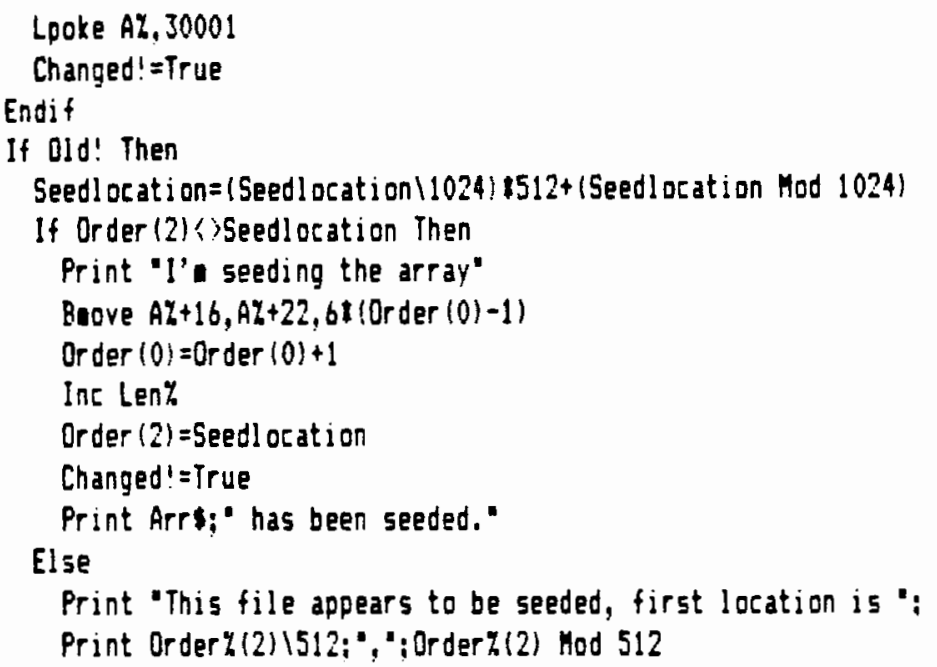

\section{Endif}

Else

If Orderq(2)<>Seedlacation Then

Print "I' seeding the array"

Beove $A \%+12, A \%+16,4 \leqslant(0$ rder $z(0)-1)$

Order $\%(0)=\operatorname{Order} z(0)+1$

Ine Len\%

Order $z(2)=$ Seedl ocation

Changed! = True

Print Arrs:" has been seeded."

Else

Print "This file appears to be seeded, first location is ";

Print Order\%(2) 11024;", "Orderz(2) And 1023

Endif

Endif

Print "Hit any key to continue."

Repeat

Until Inkey\$>

Gosub Viewarrscreen

Return

Procedure Convert

Local As

Cls

If Not 0id! Then

Print "This file appears to be converted already!"

Print "Hew for at $N="$ "Order $z(0)-1 "$ "Raax $="$ :Order $"(1)$

Else

input "Should I convert it anyway (Y or N)? ", As

$A \xi=>p$

Endif

If (Asc (As) And 223) $=89$ Then

Lpoke Notry.30001

Arrayfill Drder $2(1,0$

Print 'N $\times 1000: " \cdot$ '

For $x=0$ To Order (0)

If $x>1$ 


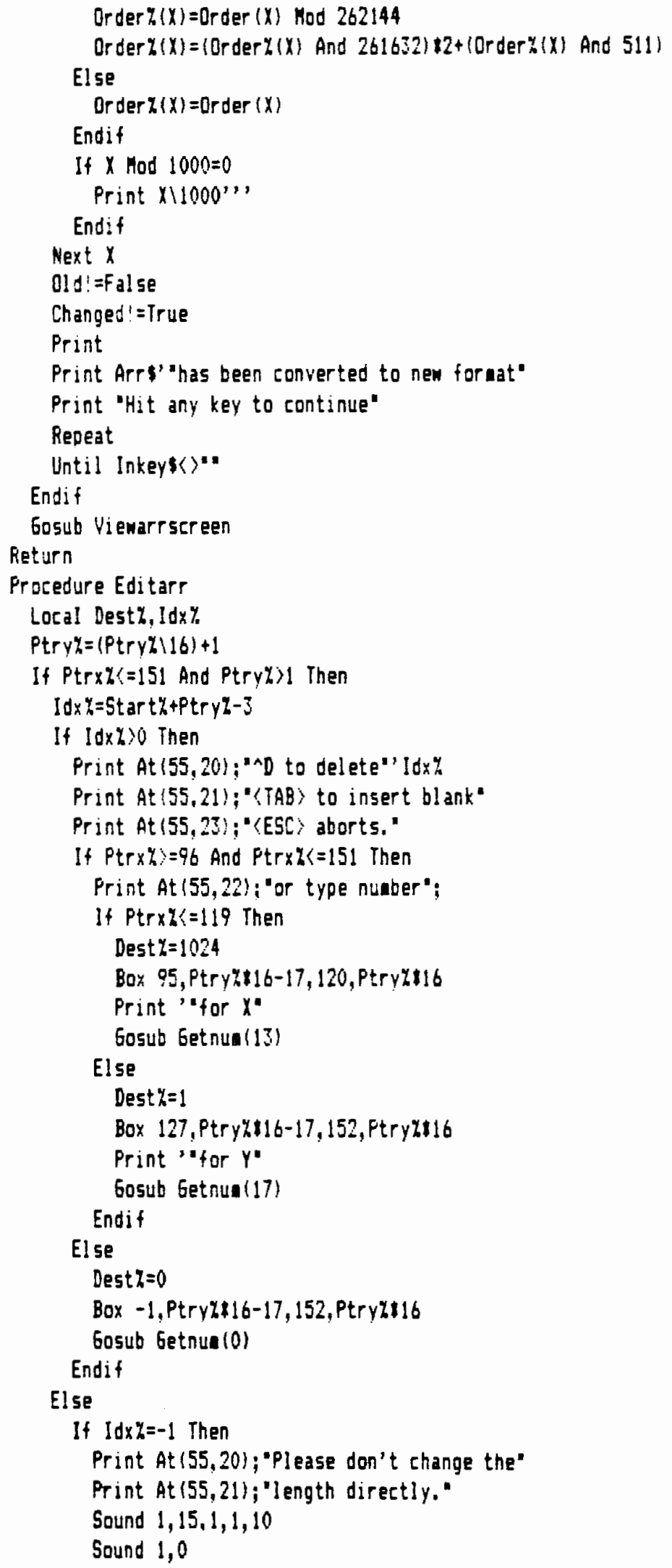




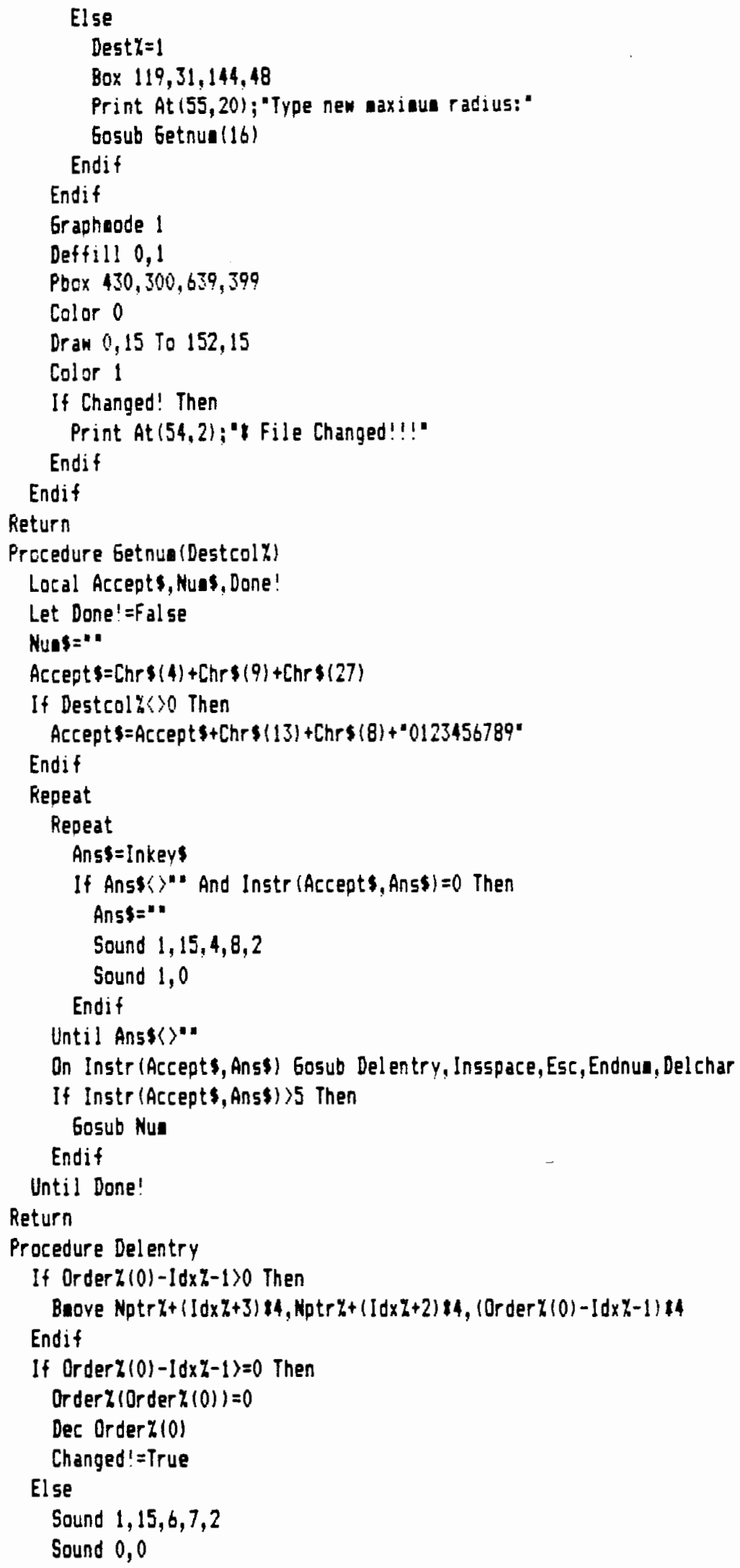




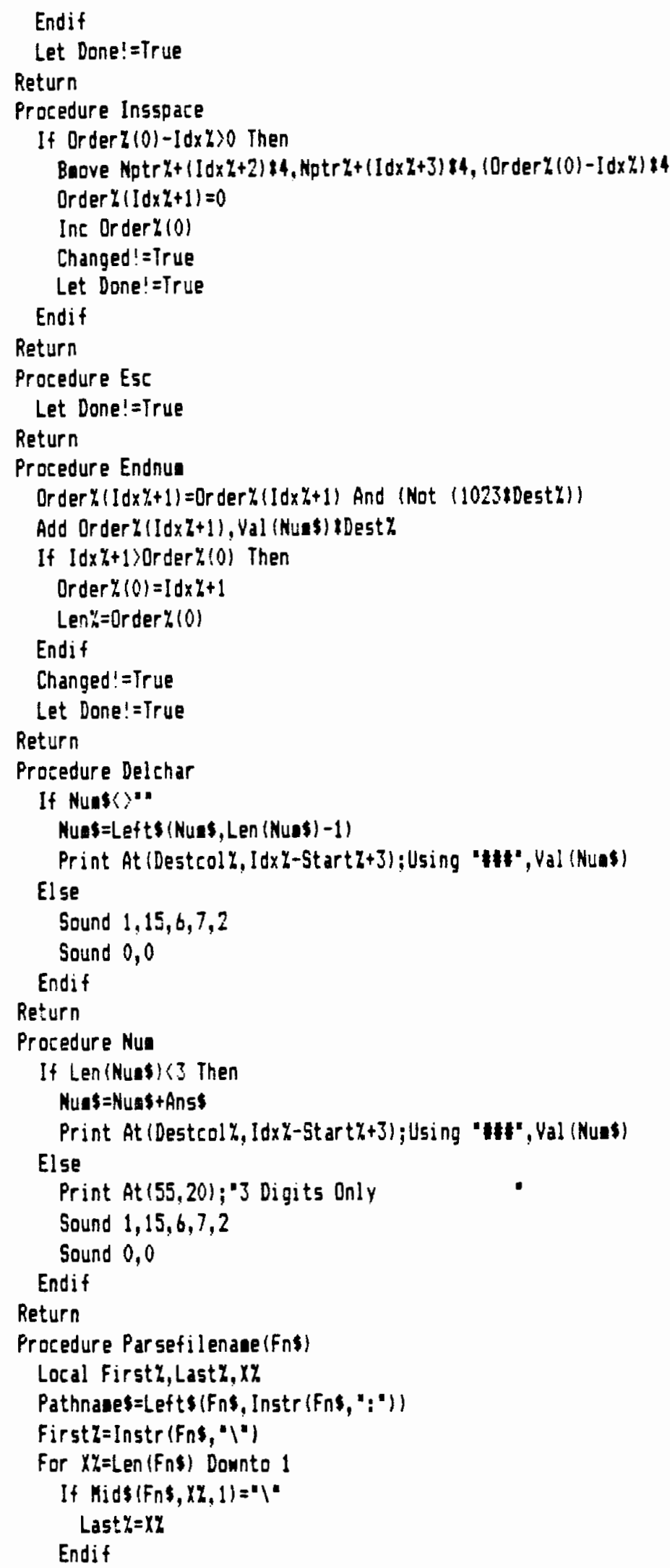


Exit If Hids(Fns, X\%, 1)="1"

Next $X \%$

Pathnase $=$ Pathnane $\$$ Hid (Fn\&,First\%,Last\%-Firsth)

File $\$=$ Mid $\$(F n \&$, Last $\%+1)$

Return

Procedure Loader

Print At $(1,3)$; 'Select array:

Fileselect "U. ARR", "SEED. ARR", File"

If Files $\rangle \cdot$ " Then

Bload Files, Lpeek (Arrptr (Order Zil))

Endif

Return

Procedure Save

Local Ay, FIy,F2y, Tlen\%

Tlen $y=$ Hin (Len $\%, 30000)$

$A$ Hotry

$\mathrm{F} 1 \%=4$

$F 2 z=8$

If 0ld! Then

Al $=90$ tr $\%$

$F 1 y=6$

$F 2 \%=10$

Endif

Print At $(32,2)$; "Saving array to" 'Arr's

Bsave Arrs, AZ, IlenZtF!\%+F2\%

Changed! $F$ al se

Gosub Viewarrscreen

Return

Procedure Changenaue

Local Teps

Tops=files

Gosub Parsefilenane (Arr\$)

Print At $(32,2)$; "File to save array to:"

Fileselect "(t.ARR', Arrs, Arrs

Gosub Parsefilenane (Arrs)

If Instr (Files,.$"$ ) $=0$ Then

Arr $\$=$ Arr\$+". ARR"

Endif

File $\$=$ Taps

Gosub Save

Return

Procedure Drawsereen

If File $\$="$ "Then

Gosub Loader

Endif

$\mathrm{Cl} 5$

Hides

Graphnode 3

Color 1

Line $400,0,400,399$

Deftext $0,16,0,32$

Text 455, 45, "Aniuator" 
Text 455,375, "Aninator"

Deftext $0,0,0,13$

Frint At $(52,1)$; Files

Text $410,77, " *$ - Reverse grouth direction"

Text 410,109, "Enter > Continue autonatic"

Text 490,125, "grouth"

Text $410,157, \therefore$ - Stop autosatic growth"

Text 410,189," ( - White background"

Text $410,221, " 1$ - Black background"

Text 410,253, 'Any other key - Single step'

Text 538, 269, 'in St op code'

Text $410,301, \%$ - Fill in to current pixel *

Text 410,333," "Undo - Exit/abort anilator"

Return

Procedure Plot (Start\%, Finish\%, Directionz, Hidth\%)

Local Wait!., S\$

$X_{h}=$ Starty

Repeat

If (Directiony>0 And $x \not\langle=F i n i s h y)$ Or (Direction\%(0 And $x y$ )=Finish\%) Then

Plot Order $y(x y)$ 11024, Order $y(x y)$ And 1023

If widthy>0 And $x y>$ width $y+1$ then

Plot Orderz(Xz-Widthz) 11024, Orderz(Xz-Widthz) And 1023

Endif

Add $x \%$, Direction\%

Endif

As=Inkeys

If Wait! Then

Repeat

$A s=$ Inkeys

Until As<>"

Endif

If $A \$\langle>$ "Then

If $A \xi=" q$ " Then

Plot Order $z(x z) 11024,0$ der $z(x y)$ And 1023

If Widthy>0 And $x y$ )

Plot Order $z\left(x z-w_{i d t h}\right) \backslash 1024$, Order $z\left(x y-H_{i} d t h z\right)$ And 1023

Endif

Swap Start\%,Finishy

nul Direction $\psi_{3}-1$

Endif

If $A s="$ " Then

Mait! $=-1$

Endif

If $A s=$ Chrs (13) Then

Hait $!=0$

Endif

If $A s=$ " ?" Then

Setcol or 0,1

Endif

If $A \xi=1$ - Then

Setcolor 0,0

Endif 
If $A s=1 /$ Then

Sget 5 s

$A \%=x \%$

Gosub Plot $(2, \times 7,1,0)$

Sput Ss

$X Y=A \%$

$A s=\cdots$

Endif

Endif

Until As=Chr $\$(0)+$ Chr $\$$ (97)

Return

Procedure Anieate

Gosub Drauscreen

Gosub Plot 2 , Order $\%(0), 1,0)$

Setcol or 0,1

Shawe

Return

Procedure Involute

Gosub Drawscreen

Gosub Fl ot (Order $\%(0), 2,-1,0)$

Setcolor 0,1

Shown

Return

Procedure Zonal

Cls

Print At $(10,12)$;

Input "Enter nuaber of pixels to display in deposition zone"; Width"

Gosub Drawscreen

Gosub Plot $(2, \operatorname{Order} \%(0), 1$, Hidth\%)

Setcolor 0,1

Shown

Return

- Coreanenu Progran

Version $=5.6$

Revdate $\$=2$ Oct $88^{\circ}$

Di Order $1(32000)$ ! Make roon for FHE arrays too.

Din Results $(1,400)$

Dis Power $\{1,400\}$

Die Std_graph:112)

Di. Menus (50)

Let Menus $(0)=$ "Desk"

Let Menus(1)=" Utilities info"

Let Henu $\$(2)="--$

For $I=3$ To 9

Let Menus (I) $=$ Str $\$(I)$

Next I

Do

Ine I

Read Menus (I)

Exit If Menus $(I)=1 \cdot \cdot$

Loop 
Data "Exit'," Buit ",", "ARR Funcs"," Autocorrelation Vectors"

Data " Mass Distribution in $X$ and $Y$ "

Data " Mass Distribution in $R$ and Theta "

Data ", "FHG Funcs"," Mass Distribution in $x$ and $y$ "

Data - Mass Distribution in $R$ and Theta -

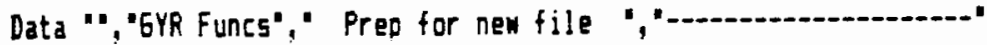

Data " Vien ?YA File"," Plot"," Regression",",","4"

Henu Henu\$1)

On Henu Gosub Handle_it_for_me

Print At $(1,3)$; "Correlation functions, Version" 'Version: ", "Revdates

Do

On Menu

Loop

Procedure Handle_it_for_ne

$\mathrm{Cls}$

If Henu $(0)=1$ Then

Gosub bive_info

Else

On Menu(0)-11 Gosub Guit, D, D, Auto, Massxy, Massrt, D, D, Fuassxy, Feassrt

If Menu(0) $>2 !$ Then

On Menu(0)-21 Gosub Dusey, D, Prep_for_new, Dusny, Vi ewdat,Pl otya, Regression

Endif

Endif

Menu Henu 11$)$

Print At $(1,3)$ :"Select function:"

Return

Procedure Give_info

Return

Procedure Quit

Menu Kill

Edit

Return

Procedure Auto

Local A\%, B\%,C\%, Ih, J\%

Gosub Loader

$\mathrm{Cls}$

If Files $\rangle "$ " Then

Input "Input n: ": $N \%$

Print "Calculating Autocorrelation vectors"

For $A \xi=2$ To Order $y(0)-N \%$

If Ay Mod $100=0$ Then

Print At $(1,6) ;{ }^{\prime} N=1 ; A \%$

Endif

$J y=\operatorname{Order} z(A z+H z)$

$I y=\operatorname{Order} \psi(A \%)$

$x j z=J z 11024$

$x_{i} y=1 \% 11024$

$Y j y=J z$ And 1023

$Y_{i} y=1 \%$ And 1023

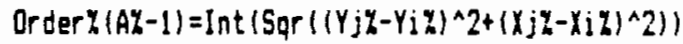

Next A\%

$\mathrm{Cls}$ 


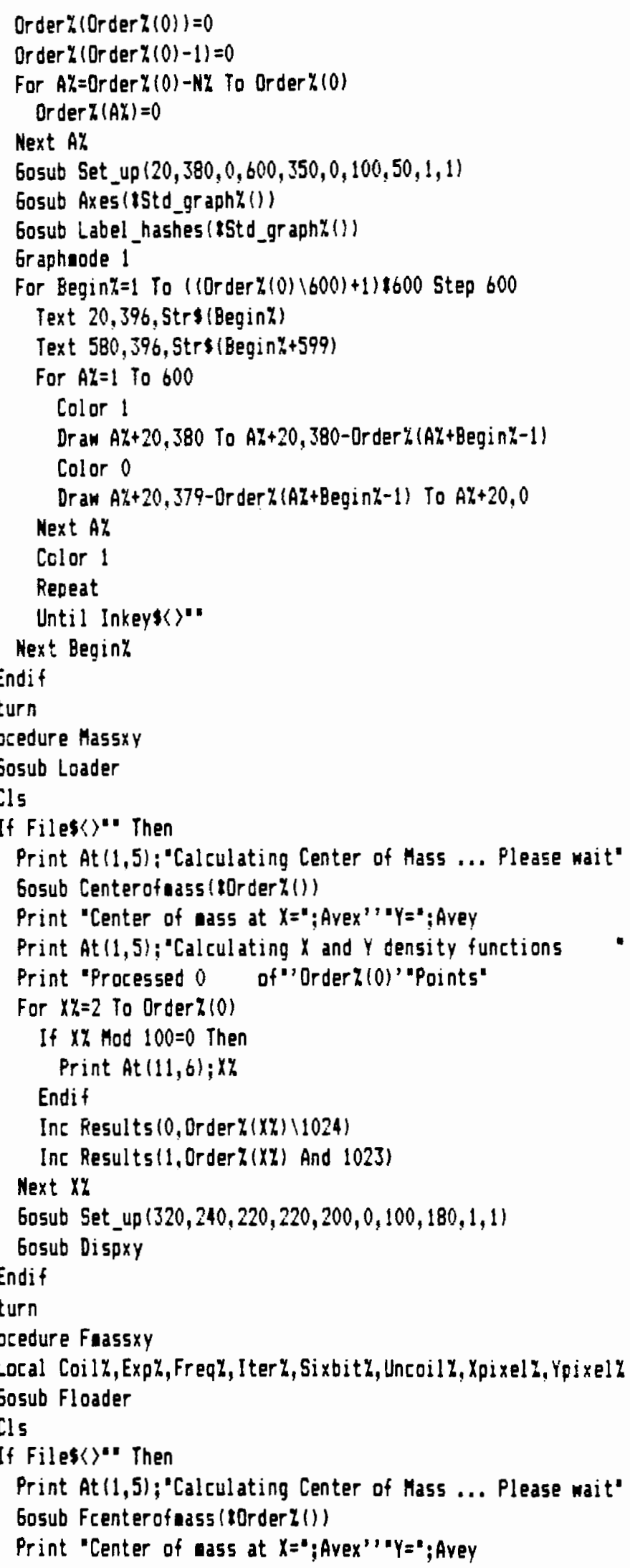


Print At $(1,5)$; Calculating $X$ and $Y$ density functions

Print "Processed 0 of 160000 Points"

For Iter $\mathrm{z}=1$ To 32000

Coil $y=($ Iter $\%-1): 5$

Freq $\%=0$ order (Iter $y$ )

If Coily Mod $100=0$ Then

Print At $(11,6)$; Coil\%

Endif

If Freq $k>0$ Then

For Sixbity=0 To 4

Exp $=64^{\wedge}$ Sixbit\%.

Frea $y=0 r d e r y(I t e r y)$ And (63tExpy)

If Freqh $\langle>0$ Then

Div Freqh, Exp\%

Uncoil $y=$ Coil $y+$ Sixbity

Xpixel $y=$ Uncoil $1 \% \backslash 400$

Ypixel $h=$ Uncoil\% Mod 400

Add Results $\left(0, x_{p}\right.$ ixel $\left.\%\right)$, Freq"

Add Results (l, Ypixel $\%$ ), Freq\%

Option "UI"

Endif

Next Sixbity

Endif

Next Iter\%

Gosub Set_up $(320,240,220,220,200,0,100,180,1,-20)$

Gosub Dispxy

Endif

Return

Procedure Dispyy

Tester:

$\mathrm{Cls}$

Graphoode 1

If Loaded! Then

Files=Dats

Endif

Gosub Parsefilenane(Files)

6osub Axesitstd_graphy(1)

Gosub Label_tashes! (5td_graphz(1)

Std_graphz $(0)=1$

Std_graphy $(3)=0$

Std_graphx(4) $=399$

Std_graphy (5) $=-200-\operatorname{Int}$ (Ayex)

Gosub Plot (tstd_graphy ())

Lbl $\$=$ "Deposit " $+F i l$ e\$+" Hass Distribution Function in $x$ "

$A$ $=40-\operatorname{Len}(\operatorname{Lb} \mid s) / 2$

Print At $(A \%, 22) !$ Lbls

Print At $(52,4)$; "Center of Mass:"

Print At $(52,5)$; Avex; ", "Avey

Gosub Cad_driver ("

Cls

Gosub Axes (t5td_graphy())

Gosub Label_hashes (tstd_graphy(1) 
Std_graph $\%(0)=1+4$

Std_graphz $(3)=0$

Std_graphy $(4)=399$

Std_graph $(5)=-200+$ Int (Avey)

Gosub Plot (1Std_graph\%(1))

Lbl \&=Left $(L b \mid \$$, Len $(L b \mid \$)-1)+" y$ c

Print At $(A \%, 22)$; Lb1s

Print At $(52,4) ;$ "Center of Mass:"

Print At $(52,5)$; Avey;", "Avey

Gosub Cod_driver (")

\section{Return}

Procedure Massrt

Local A\%, B\%, RY, Thy, Rav, Ro\%, Fho\%

Graphoode 1

Gosub Loader

$\mathrm{Cls}$

If File $\$\langle$ " Then

Print At $(1,5)$; 'Calculating Center of Mass ... Please wait"

Gosut Center of ass (180rder\%(1))

Print "Center of nass at $x=$ ": Avex" " $y=$ "; Avey

Print At $(1,5) ; " C a l c u l a t i n g ~ R$ and Theta density functions "

Print "Frocessed 0 of "Ordery(0)" "Points"

For $x y=2$ To Order $y(0)$

If $x \%$ Hod $100=0$ Then

Print At $(11,6) ; X \%$

Endif

$A \%=0 \operatorname{rder} z(x z) \backslash 1024$

$B \%=\operatorname{Order} y(x \%)$ And 1023

$R Z=\operatorname{Int}\left(S q r\left((A z-A v e x-200)^{\wedge} 2+(B \%+A v e y-200)^{\wedge} 2\right)\right)$

Th\% $\%=\operatorname{Trunc}(A \operatorname{tn}((B \%+A v e y-200) /(A \%-A v e x-200+0.00001)) 157.3)$

Add Th\%, 180

If A\%-Avex-200;0 Then

Add Thz, 180

Endif

If Thy $)=360$ Then

Sub Th\%, 360

Endif

Inc Results $\left(0, R^{4}\right)$

Inc Results (1, Thy)

Next $x y$

Gosub Set_up $(100,250,0,220,220,0,50,100,1,1)$

Gosub Disprt

Endif

Return

Procedure Faasst

Local Coil , Exp\%, Freq\%, Iter $\%$, Sixbit\%, Uncoil $\%$, Xpixel\%, Ypixel\%

Gosub Floader

$\mathrm{Cls}$

If Files(>" Then

Print At $(1,5)$; 'Calculating Center of Mass ... Please wait'

Gosub Fcenter of ass (10rder $y(1)$

Print "Center of ass at $X=" ;$ Avex" "Y=";Avey 
Frint At(1,5): "Calculating $k$ and Theta density functions "

Print "Processed 0 of 160000 Points"

For Itery $y=1$ To 32000

Coil $z=(1 \operatorname{ter} \%-1) 15$

Freq $y=$ Order $\%($ Iter $\%)$

If Coil\% Mod $100=0$ Then

Print At $(11,6):$ Coil\%

Endif

If Freqkiso Then

For Sixbity=0 To 4

Exp $=64 \%$ Sixtit\%

Freq" $=0$ ordery (Itery) And (6T)Exph)

If Freq?yo Then

Div Frea\%, Exp\%

Uncoil $\%=$ Co: $1 \%+5 i x$ it $\%$

Xpixel $y=$ Uncoil $\% 1400$

Ypixely= Uncoil 1 : Hod 400

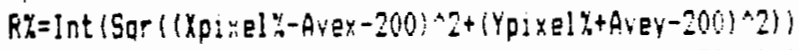

Thy=Trunc (Atn (lYpizel $\%+A v e y-200) /($ kpixel $\%$-Avex-200+0.00001) 157.3)

Add Th\%, 180

If xpixely-Avex-200<0 Then

Add Th\%, 180

Endif

If Thy $\rangle=360$ Then

Sub Thy, 360

Endif

Add Results $(0$, , F $\%)$,Frea\%

Add Results (!, Th\% , Freq\%

Option "U1"

Endif

Mext Sixbity

Endif

Next Iter\%

Gosub Set_uo $(100,250,0,220,220,0,50,100,-20,-20)$

5osut Disprt

Endif

Return

Procedure Disprt

$\mathrm{Cls}$

If Loaded! Then

Files $=$ Dats

Endif

Gosub Farsefilenane(file\$)

Testing:

Scl $\times y=$ Std_graphy (9)

Std_graphy $(9)=1$

Gosub Axes (tstd_graphy())

Gosub Label_hashes (t5td_graphy(1))

Std_graph $z(\overline{0})=1+8$

Std_graphz(3) =0

Sto_graph $x(4)=201$

Std_graph $y(5)=0$ 
Gosib Plot (tstd_granh\%())

Lbl $\$=$ "Deposit "+File $\$+$ "Mass Distribution Function in R."

$A_{h}^{4}=40-\operatorname{Len}(\operatorname{Lbl} \$) / 2$

Print At $(A \%, 22)$; LbI

Print At $(52,4)$; "Center Of Mass:"

Print At (52,5); Avex:' ", 'Avey

Print At $(52,6):$ Chr $(255): "="$ 'Ave

Graphaode 3

Text 408,93, "r"

Gosub Baror aph!100+Ave, 250,0,-20)

Gosub Bargraph(100+Moden, 250,0,-28)

Graphacde 3

Deftext $1,0,0,6$

Text $100+$ Ave $-?, 250+20+10, " r$ "

Text 100+Ave-3, 250+20+9, Chr $\$(255)$

Text 100+Hodey-15,250+20+18, "aode"

Deftext $: 1,0,0,13$

Graphoode !

Gosub Cod_driver ("r")

$\mathrm{Cls}$

Gosut Set_up $\left(220,200,180,180,180,180,150,150,50 ! h_{4}\right.$, std_graph\%(10)!

Gosub Axes (15td_graphy())

Gosut Label_hashes (t5td_graph\% (i)

Std_graphs $(\overline{0})=1+4+32$ !1 color, 4 ufper, 32 polar

Std_graphy $(3)=0$

Std_graphy $(4)=360$

Std_graph\%(s) $=0$

Gosub Plot (tstd graph\%(1)

Lbl s=Lefts (Lb!s, Len (Lbis)-1)+" Theta"

$A Z=\operatorname{Len}(L b l s)$

Print At $(52,2)$; Lefts (Lb) $1, A \%-35)$;

Print At $(52,3)$; Hids (Lb $\left.15, A_{6} \%-34,17\right)$

Print At $(52,4)$; Pight $\$(L b 15,17)$

Frint At $(52,6)$ : "Center of Mass:"

Print At $(52,7)$; Avex; ', "Avey

Gosub Cadodriver(")

Let Loaded! $=$ True

Len $\%=360$

Return

Procedure Cad driver (Char $\$$

Local Char\%

Char $\%=-(\operatorname{Char} \$\langle\rangle)^{n)}$

Do

Gosub Falinel"S = Sooothing $H=$ Modes $E=$ Edit Screen (ESC) aborts (CF) stores screen")

Repeat

As $=$ Inkeys

Until As \langle\rangle $"$ "

Exit If $A s=C h r \$(27)$

If (Asc (As) And 95) $=83$ Then

On Chary 6osub Label_ave

Gosub Suoothing

On Chary Gosub Label_ave 


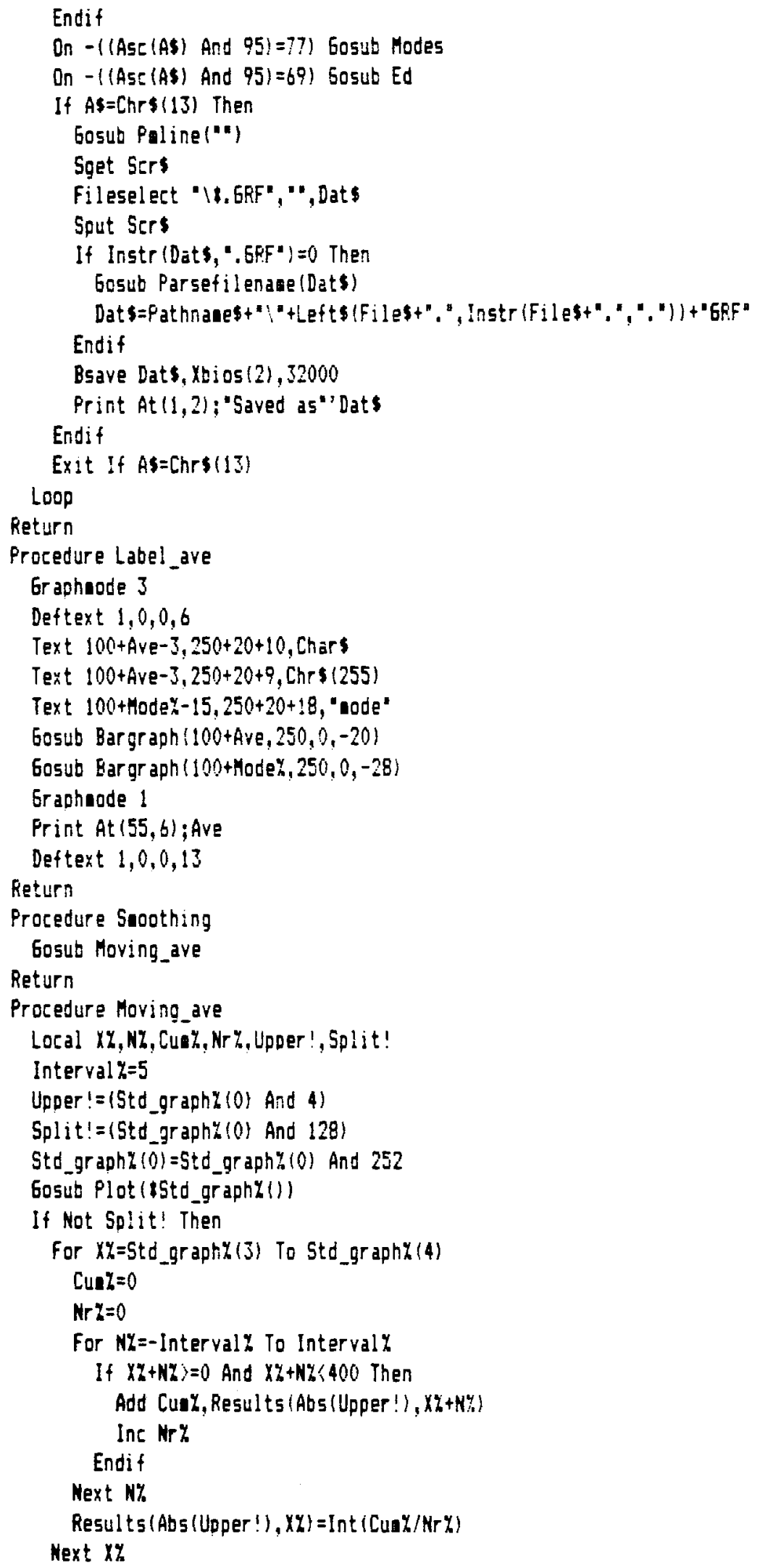


Else

Endif

Add Std_araph\% (0) , 1

Bosub Plot (15td_graphz))

Return

Procedure Ed

Local $X, Y, K, A$ s

Gosub Paline ("Lleft Button〉 - Add Text (Right Button> - Move Area (ESC) exits")

Repeat

Mouse $X, Y, K$

On $K$ Gosub Text, Move

If $K$ Then

Gosut Paliriel"(Left Button〉 - Add Text (Right Button〉-Move Area (ESC) exits") $k=0$

Endif

As =Inkey

Until $(A s=$ Chrs $(13))$ Or $(A s=C h r s(27))$

Return

Procedure Preo_for_new

Let Loaded! =False

Dats $="$ "

Return

Procedure Viewdat

Loca! Start\%, Line\%,AS, OId!,Optr\%,Nptr\%

Optry=Lpeek (Arrptr (Results ())!)

If Not Loaded! Then

Gosub Dloader

Endif

If Dats?" Then

Gosub Viendatscreen

Do

For Line $=$ =5tart\% To Start $\%+23$

If Liney 401 Then

Print At $(1$, Liney-Start\%+2); ising " H\# "Line\%+Base\%;

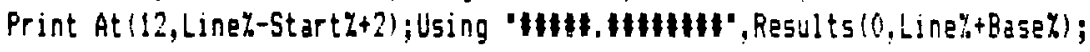

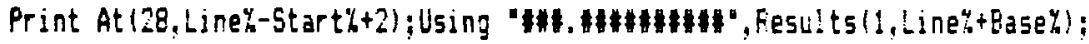

Endif

If Liney $>400$ Then

Print At (1,Line\%-Starty+1);"

Endif

Next Linez

Repeat

As $=$ Inkeys

Until As<>"

If $A s=C h r \$(13)$ Or $A s=C h r s(32)$ Or As=Chrs(0)+Chrs $\$(80)$ Then

Add Starty,25

If Starty>375 Then

St art $y=375$

Endif

Endif

If $A s=C h r s(0)+\operatorname{Chr} s(72)$ Then

Sub Start\%, 25 


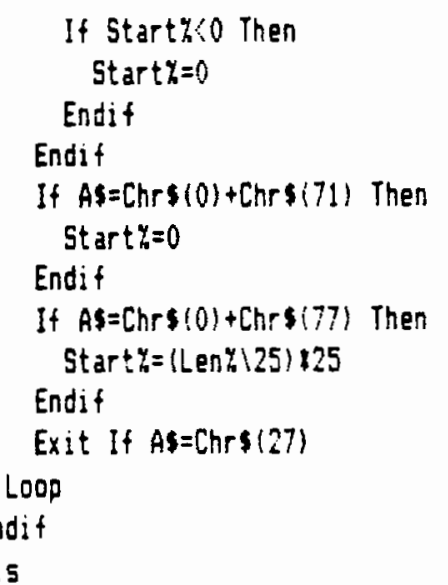

Returin

Procedure Viewdatsereen

Cls

Box $384,75,600,185$

Text 392,93, "Up arrow - Page up"

Text 392,109, "Down arrow - Page down"

Text 392,125, "〈Space〉, (CR) - sane as Down arrow"

Text 392,141, "Hone' - Top of array"

Text 392,157, "Left arrow - Last page of array"

Text 392,173, " $\langle$ Esc $\rangle$ - Main senu"

Start $y=0$

Print At $(1,1) ;$ "N of "Len"

Print At $(12,1)$ : Zeros;

Print At $(28,1)$; Ones;

Print At $(49,1)$; Dats

Return

Procedure Plotya

If Not Loaded! Then

Gosub Dloader

Endif

If Dats $>$ " Then

If Typ $s=$ 'XYA' Then

Gosub Di spxy

Else

If Typs="RYA" Then

Gosub Disprt

Else

$\mathrm{Cls}$

Gosub Axes (tsto_graphy(1)

Gosub Labe!_hashes (tstd_graphz(1))

Gosub Flot (t5td_graph\%())

Gosub Regression

Gosub Cad_driver(")

Endif

Endif

Endif

Return

Procedure Regression 
Loca! Cutoff, Cutoffl

If Not Loaded! Then

Gosub Dloader

Endif

If Dats $>$ " Then

Power $(0,0)=0$

Input "Lower cutoff for regression $(L n(N)$ in linear region, 0 for all) "; Cutoff

If Cutoff $=0$ Then

Cutoff $y=1$

Else

Cutoff\% $=$ Int $\left.\left(\left(82 t E_{x p} \text { ! Cutoff }\right)\right)^{\wedge} 0.41666666667\right)-10$

Endif

For N\%=Cutoffy To Len\%

Ine Power $(0,0)$

Power $(0$, Power $(0,01)=$ Results $(0$, N\%)

Power $(1$, Power $(0,01)=$ Fesults $(1, N \%)$

Next N\%

Gosub Power

Print " $0=" 11 /$ Slope

Print "File = "idats

Endif

Return

Procedure Loader

Print At (1, 3); "Select array:

Filesel ect "I" ARF", "SEED. ARR", Files

If Files?" Then

Arrayfill Results (1),0

Arrayfill Orderzll,0

Bl oad Files, Lpeek (Arrptr (Orderz()))

Endif

Return

Procedure Floader

Print At $(1,3)$; "Select array:

Fileselect "It.FHG", "LONGLIST. FHG", Files

If Filek $\rangle "$ "Then

Arrayfill Results (1,0

Arrayfill Order $\%$ (), 0

Bload Fil es, Loeek (Arrotr (Ordery (1))

Endif

Return

Procedure Dl oader

Local T\$\$, To $\$$

Do

Print At (1,3); "Select array:

Fileselect "It.?YA"," Dats

If Dat $\rangle "$ "Then

Tf $\$=$ Files

Tps=Pathnaes

Gosub Parsefilenane (Dat\$)

Typ $\$=$ Mid (Files, Instr (File\$,.$")+1)$

Datpath $\$=$ Pathnanes

Pathnames $=$ Tps 
Files $=$ Tf

Endif

Exit if Instr ("PYAgYaXYaRYA", Typ 130 Or (Dat $\$="$ )

Print At $(1,1)$; Dats' is an unknom tyoe of data file. Please"

Print "enter file with .FYA, .GYA, .XYA, or . RYA extension."

Loop

If Dats $>\cdots$ Then

Arrayfill Results 11,0

Bload Dats, Lpeek (Arrotr iResults(i))

Let Loaded! =True

If Instr("PG', Lefts(Typs, 1)) >0 Then

Len $y=$ Results 10,0$)$

Base $\%=1$

Split! $=$ True

If TYp $=$ "PYA" Then

Zeros="Radius of zone"

$X s=' \ln R$ '

Let Ones="Filled Area"

$Y \xi=" \operatorname{Ln} C(R) *$

Gosub Set_up $(100,300,0,430,230,0,50,50,50,50)$

Std_graph\% $(0)=1+64+128+256$

Std_graph\%(3)=Ease\%

Std_graph\% $(4)=$ Len $\%$

Std_graph' $(5)=0$

Else

Zero $\$=" 1$ of Deposits"

$X s=" \operatorname{Ln} N "$

Let Ones="Kadius of Gyration"

$Y s=" \operatorname{Ln} R g$ "

Gosub Set_up $(100,300,0,430,230,0,40,40,40,40)$

Std_gr aphy $(0)=1+64+128+256$

Std_graphy (3) =Base\%

Std_graphy (4)=Len\%

Std_graphy $(5)=0$

Endif

Else

Base $z=0$

Split! = False

If TYO $\$=$ "XYA" Then

Len $y=400$

Len $0 \%=400$

Len $1 \%=400$

Zeros: "Density in $x$ "

Let One $=$ "Density in $Y$ "

$x:=>x^{\prime \prime}$

$Y s=$ 'Density in $x^{\circ}$

Gosub Set_up $(320,240,220,220,200,0,100,180,1,1)$

Std_graphy $(0)=1$

Std_graphy(3)=Basel

Std_graphy $(4)=$ Len\%

Std_graph $\%(5)=0$

Endif 


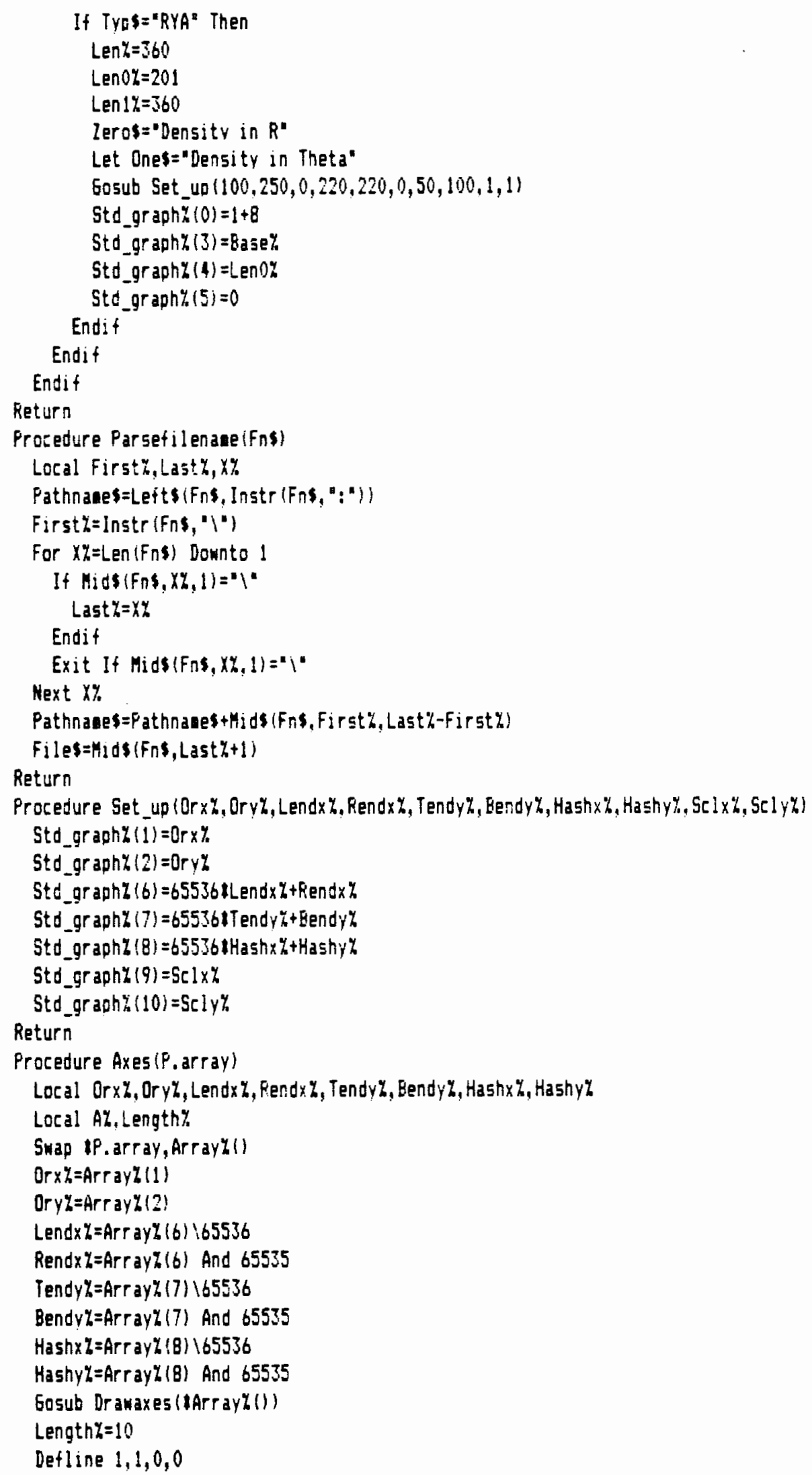


If Hashxusio Then

For $A \%=0 r x \%$ To Or $x y$-Lendx\% Step -Hashx"

Dr aw A\%,Ory\%-Length\% To Az, Ory\%+Length\%

Next $A$ Y

For $A \%=0 r x y$ To Or $x_{n}^{y}+$ Rendx\% Step Hashxy

Draw A\%,Oryz-Length\% To Ah, Ory\%+Length\%

Next $A \%$

\section{Endif}

If Hashyzi>0 Then

For $A \%=0 r y \%$ To Ory\%-Tendy\% Step - Hashy\%

Dr aw Orxy+Lengthy, Ay To Orxy-Length $y_{\text {, }}$ \%

Next Ay

For $A \%=$ Ory\% To Oryz+Bendy\% Step Hashy\%

Draw Orxy+Length\%, A\% To Orxy-Length\%,A\%.

Next A\%

Endif

Swap IF. array, Array?()

Return

Procedure Dramaxes /P.array!

Local Orx $\%$, Ory $\%$, Lendx $\%$, Rendx $\%$, Tendy $\%$, Bendy, Hashx\%, Hashy\%

Swap IP.array,Arrayz)

Orxy =Arrav:(1)

Ory:=Arrayz(2)

Lend ${ }^{2} h=$ Arr av $\%(6) 165536$

Rendx $y=\operatorname{Arr}$ ay $\%(6)$ And 65535

Tendy $\%=$ Array $\%(7) 165536$

Bendy $\%=$ Array:(7) And 65535

Defline $1,1,1,1$

If Lend $x=0$ Then

Defline $1,1,0,1$

Endif

If Rendx $h=0$ Then

Defline $1,1,1,0$

Endif

Draw Or $x \%$-Lendxy, Ory To Or $x \%+$ Rend $x \%$, Oryz

Defline $1,1,1,1$

If Tendy $y=0$ Then

Defline $1,1,0,1$

Endif

If Bendy $=0$ Then

Defline $1,1,1,0$

Endif

Draw Orxy, Oryz-Tendy\% To Or $x y_{,}$Ory $y+$ Bendy $\%$

Swap (P.array, Array (1)

Defline $1,1,0,0$

Return

Procedure Label hashes (P. array)

Local Hashz, Loendy, Hi endz, Scal e, AZ, Lbl

Swap tP. array, Arrayz()

Deftext $1,0,0,6$

Hashy=Array\% (8) 165536

Loendz=Arr ayh(6) 165536 
Hiendz=Arravz(6) And 65535

Scal e=Arrayz(9)

If Scale<0 Then

Scal e $=-1 /$ Scale

Endif

$A \%=H a s h$ ! To Hiendy Step Hash"

While AYCHiendy

Lbl $\$=\operatorname{Str} \$(A z / S c a l e)$

Text Array\%(1)+A\%-Len (Lb1 \$) 44 , Array\%(2)+18,Lbls

If AzLLoend\% Then

Text Arrayz(1)-A\%-\{Len (Lb1 $\$)+1) 14$, Arrayz(2)+18, $x-4+L E 1 \$$

Endif

Add A\%, Hash?

Wend

Hashy=Array\%(8) And 65535

Hi end? =Arr ay $2(7) \backslash 65536$

Loend" $=$ Arrav" $(7)$ And 65535

Scal $e=$ Arr ay $y(10)$

If Scalec 0 Then

Scale $=-1 /$ Scale

Endif

$A \%=H a \operatorname{sh} \%$ ! To Hiend\% Step Hash\%

thile AKrHiendy.

Lbl $s=\operatorname{Str}(A \mathrm{~A} / \mathrm{Sc}$ al e)

Text Arrayz(1)-10-Len (Lb] 1$)$ 18, Arrayy (2)-A\%+4,Lb)

If Ak:Loendy Then

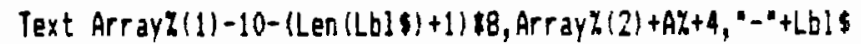

Endif

Add A\%, Hash\%

Wend

Swap IP. array, Arrayz ()

Deftext $1,0,0,13$

Return

Procedure Plot (P.array)

Local Flags\%, Upper!, Collect!, Xplot!, Pol ar!, Line!, Split!, Logs!, Count\%

Local Datur, Indep, Xbeg\%, Xend $\%$, Ybeg\%, Yend\%, Max $\%$, Sun $\%$, Nave\%.

Local Sclx, Scly

Swap 1P. array, Arrayz)

Fl ags\%=Arrayz 10 )

Upper! $=F l$ ags\% And 4

Collect! =Flags\% And 8

Xplot! $=51$ ags\% And 16

Polar! =Flagsh And 32

Let Line! =F! ags* And 64

Split! $=F !$ ags\% And 128

Logs! =5lags\% And 256

Sc $x=$ Arr ayz $(9)$

Scly=Arrayz(10)

If Sc] $x<0$ Then

Sel $x=-1 / \operatorname{Sc}] x$

Endif

If Scly<0 Then 


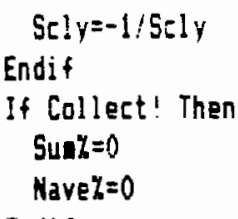

Endif

Color Flags\% And 1

Graphuode (Flags" And 2) +1

For Count $\%=$ Arrayz(3) To Arrayz(4)

If Split! Then

Indep=Resul $t s(0$, Count $\%)$

Datum=Resul ts (1. County)

Else

Indep = Count\%

Datua =Resul ts (Abs (Upper!), County)

Endif

If Logs! Then

Indep $=\log (\operatorname{lndep})$

Datun=Log (Datun)

Endif

If Not Polar! Then

Xteg\%=Array:(1)+Abs (xplot!+1) (Indep $15 c$ (x+Array $(5))$

Ybeg\%=ArravZ(2)+Abs (Xplot!) (Indep (Se) y+ArrayZ(5))

$X$ endy $=0$ atuatScl $x$ tAbs (Xplot!)

Yend\%=Datuatsel Y Abs $\left(X_{0} l o t !+1\right)$

El se

$x$ beg $\%=\operatorname{Array} z(1)$

Ybeog $h=A r r a y z(2)$

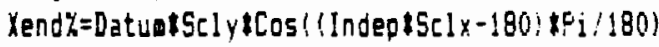

Yend $z=-D$ atualSclytSin $($ Indep $(S c] x-180)$ if $1 / 180)$

Endif

If Line! Then

If Count? $=$ Array $(3)$ Then

Dram xbeg\%+xendy, ybeg $\%$-yendy

Else

Draw To Xbeg $\%+x e n d z, Y b e g \%$-Yendy

Endif

Else

Dr an Xteg\%, Ybeg\% To Xbegh+Xend\%, Ybeg\%-Yend\%

Endif

If Collect! Then

If Datury) Max I Then

Hax : = Datus\%

Mode $\%=$ Count $\%$

Endif

Add Sun\%, Results (Abs (Upper !), Counth) County

Add Nave\%, Results (Abs (Upper! i, County)

Endif

Next County

If Collect! Then

Ave=Sun\%/Nave\%.

Endif 
If ((F) ags

Color 1

Graphoode 1

Gosub Drawaxes (\&Arrayz(1))

Endif

Swap IF. array, Arrayä()

Return

Procedure Bargraph (Xbeg\%, Ybeg\%, Xend $\%$, Yendz)

Draw Xbeg\%, Ybeg\% To Xbeg\%+Xend\%, Ybeg\%,

Return

Procedure Po! ine(Txt)

Deftext $1,0,0,6$

Graphoode 1

Tent 0,398, Space $(80)$

If Txtsy" Then

Graphoode 4

Txt $=$ Spaces (40- (Len (Txt5) $) 2))+T x+5$

Text 0,398, Txt $\$+5 p$ aces $(80-\operatorname{Len}(T x+s))$

Endif

Graphoode 1

Show:

Return

Procedure Text

Local $X, Y, K$, In\$, Titles, Mses, Bi gose\$, Sers, Si ze\%, As

Size $=6$

Gosub Faline("〈Left) - Locate text line T - Toggle print Size 〈ESC〉 aborts")

Deftent $1,0,0,5 i z 2 \%$

Graphode 1

Mses=Hk1 $\$(393224)+H k 1 \$(65536)+H k i s(1)+H k 1 s(2080412160)+H k 1 \$ 1811949568)$

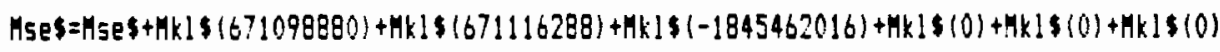

Hses=Hses+Hkl $(27648)+\mu k l s(268439552)+m k 1 s(268439552)+m k l s(268439552)$

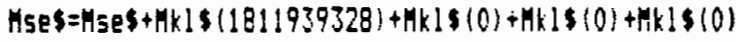

Bignse $=M k l \$(65537)+H k i s(1)+m k 1 s(1)$ ! Ref at 1,1 ; filler; standard colors 0,1

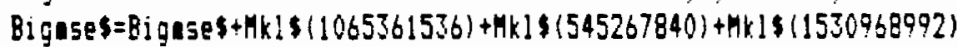

Bi guses=Bi geses+mkl $(-1147108448)+m k(s(-1079984224)+m k 1 s(1598038144)$

Bigeses=Bigise\$+Hk1 $\$(\$ 45267840)+H k 1 \$(1065353216)$

Bi getse $s=$ Ei gases+Hk1 $\$(7936)+H k 1 s(520101632)+4 k 1 s(612385856)+H k 1 s(1145076800)$

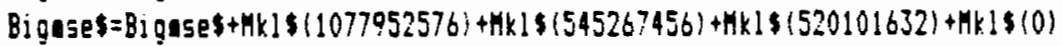

Defnouse Mse

Repeat

Unti! Mousek=0

Repeat

House $X, Y, K$

As=Inkeys

If (Asc (AS) And 95 ) $=84$ Then

Sizey $=-(5 i z e z-9.5)+9.5$

If Sizey $=13$ then

Defuouse Bignses

El se

Defnouse Mses

Endif

Deftext $1,0,0$, Sizex 


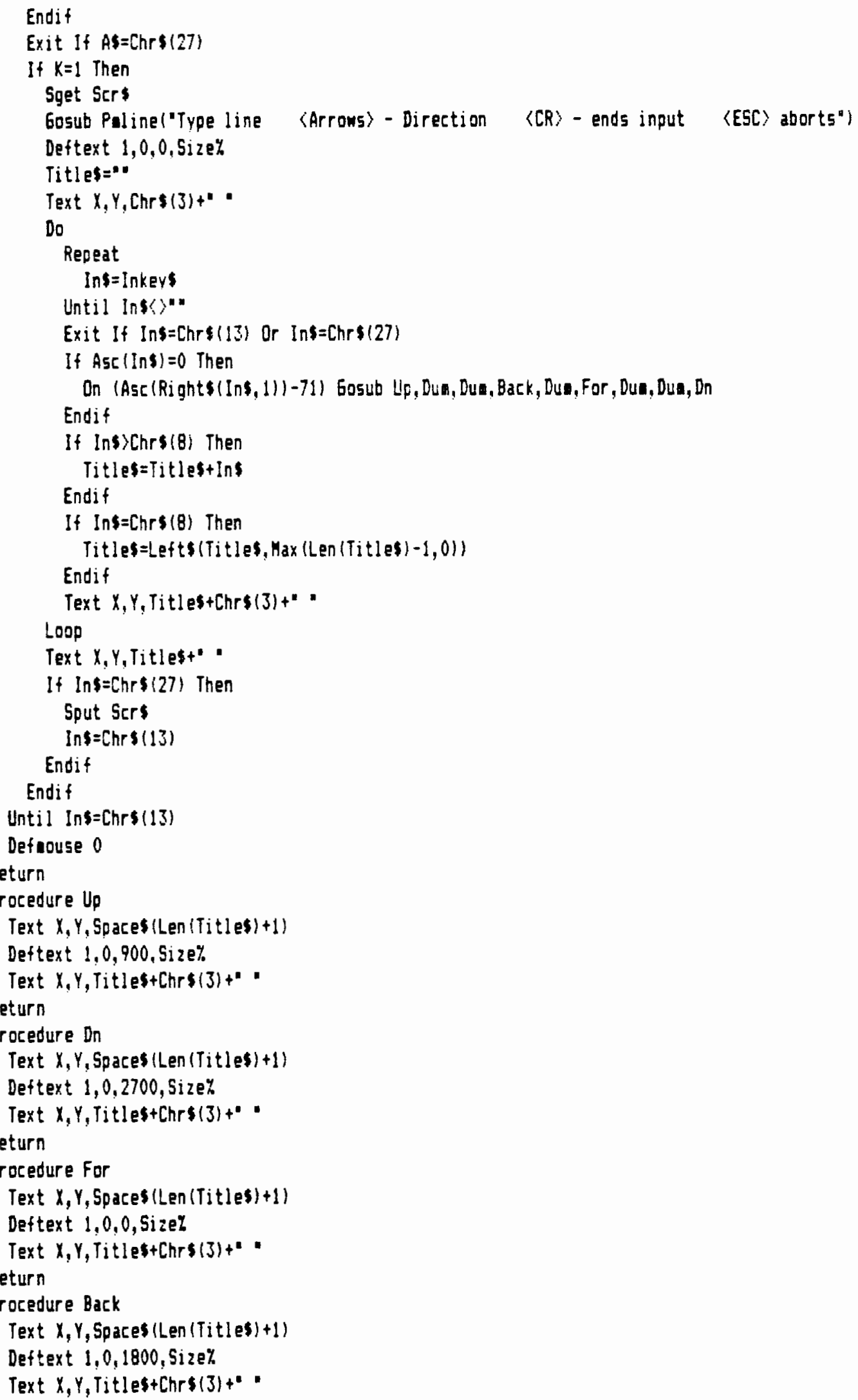


Return

Procedure Move

Local X, Y, K, As, Hses, XO\%, YO\%

Mouse $X, Y, K$

Gosub Puliner"〈Right> - opens box, rel ease records area.")

Graphaode 3

$x 0 \%=x$

YoY $=Y$

While $k=2$

Mouse $X, Y, K$

Box $\times 0 \%, Y 0 \%, X, Y$

Box $10 \%, Y 0 \%, X, Y$

Wend

Get $X 0 \%, Y 0 \%, X, Y, H s e s$

$x O x_{2}=\operatorname{Hin}(X 0 \%, X)$

Yo\%=Hin $(Y 0 \%, Y)$

Put Xo\%, Yo\%, Mses, 6

Gosub Palinel" $\langle$ Left - Places area C - Copies area D-Deletes area 〈ESC〉 aborts")

Hidew

Grapheode :

Do

House $X, Y, K$

Put $X, Y, Y$, Hse $\$, 6$

$A \xi=\operatorname{lnkeYs}$

If (Ase (As) And 95) $=67$ Then

Put $\times 0 \%, Y 0 \%, 458 \$, 7$

Endif

If (Asc (As) And 95$)=68$ Then

Put X,Y,Hses, 6

$K=1$

Endif

If $A \&=C h r \$ 127)$ Then

Put $X, Y, M s e \$, 6$

Put $X 0 \%, Y 0 \%, M 5 e \$, 7$

$k=1$

Endif

Exit If $K=1$ Or Mousek=!

Put $X, Y$, Hses, 6

LoOp

Shoms

Repeat

Until Mousek =0

Return

Procedure Center of nass (P. array)

$N z=0$

Swap 19 array, Avearrayzl)

Print "Processed 0 of "Avearrayz(0)" "Points"

Avex =Ave arrayz(2) 11024

Avey=Avearray (2) And 1023

Do

Ine $N \%$

If N2 Mod 100=0 Then 
Print At $(11,6): N \%$

Endif

Exit If Avearrayz $(N z+1)=0$ And Avearrayz(Nz+2) $=0$

Avex $=($ Avex $($ (Nz-1) $)+($ Avearrayz $(N z+1) 11024)) / N \%$

Avey $=($ Avey $(N z-1)+($ Avearrayz(KZ+1) And 1023$) 1 / N z$

Loop

Sub Avex, 200

Avey=200-Avey

Swap IP. array, Avearrayz)

Return

Procedure Fcenterofeass (P.array)

Swap IP. array, Avearraył ()

Print "Processed 0 of 160000 Points"

County $=0$

$\mathrm{N} \%=0$

Repeat

Inc NY

Until Avearray'h(N\%)<>0

Sixbity $=0$

While (Avearrayz(NY) And (63464^Sixtity)) $=0$

Inc Sixbity

Hend

Uncoil $\%=(N \%-1) 15+$ Si $\times$ bit $\%$

Exp $y=64^{\wedge}$ Si $x$ bity

Freq $\%=($ Avearray $\%$ (NY) And (63tExp\%))/Exp\%

Avex $=($ Uncoil $\% \backslash 400)$ \&Freq\%.

Avey=(Uncoily Mod 400) IFreq\%

Add Counth, Freq\%

Inc Sixbity

Repeat

Exp $=64^{\wedge}$ Sixbity

Freq $=$ (Avearrayz(Ny) And (63tExph) $) /$ Exp\%

If Frequ>0 Then

Avex $=($ Avex $x$ Count $\%+($ Uncoi $1 \%)$ 400) Ifreq $\%) /($ Count $\%+$ Freq $\%)$

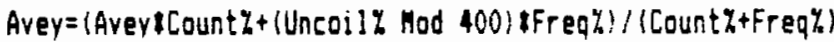

Add Count\%, Freq\%

Endif

Ine Sixbity

Unti] Sixbit $x>4$

Ine $\mathrm{NH}$

Repeat

Coil $x=(N z-1) 15$

Freq $h=$ Avearrayl (NZ)

Uncoil $y=$ Coil $1 \%+$ Sixbit $y$

If Uncoily hod $100=0$ Then

Print At $(11,6)$; Uncoily

Endif

If Freqki>0 Then

For Sixbity $=0$ To 4

Expz $=64^{\wedge}$ Sixbity

Freoz=Avearrayz(Nz) And (638Expy)

If Freaz<>0 Then 


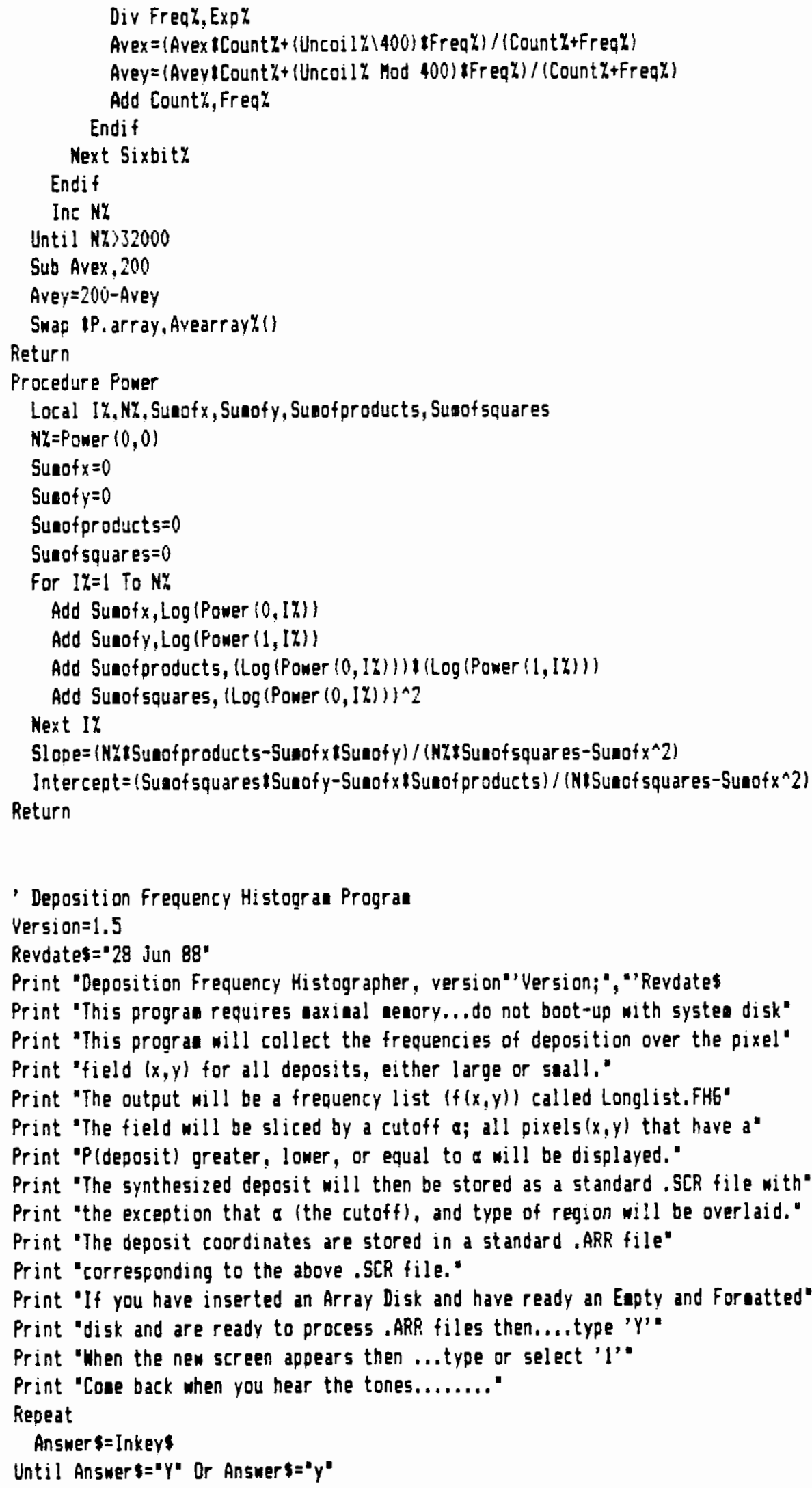




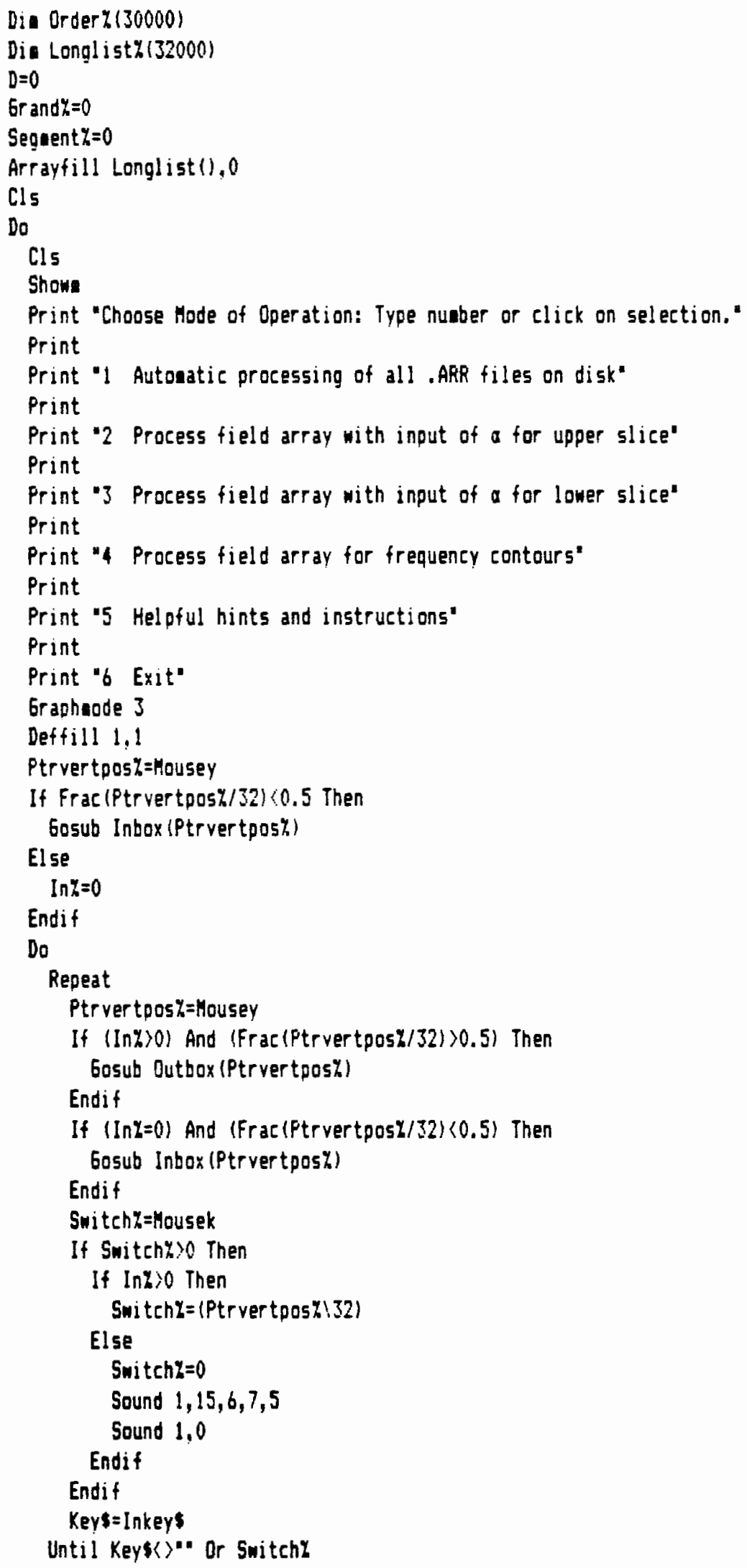


If Switch\% Then

Keys $=5 \operatorname{tr} \$$ (Switchy)

Endif

Exit If Val (Keys) $>0$ And Val (Keys) $<7$

Sound $1,15,6,7,5$

Sound 1,0

Loop

$\mathrm{Cls}$

Grapheode 1

On Val (Key\$) Gosub Array, Upper, Lower, Contour, Help, Exit

$\ln y=0$

Switch: $=0$

Loop

End

Procedure Inbox (Ht $\%$ )

$\mathrm{Ht} \%=321(\mathrm{Ht} \%(32)$

If Hty>16 And $H t \% 224$ Then

Pbox $-1, H t \%, 500, H t \%+16$

Inz=Ptrvertpos\% 132

Endif

Return

Procedure Outbox (ht $\%$ )

$H t y=321 \operatorname{In} \%$

Pbox $-1, \mathrm{Ht} \%, 500, \mathrm{Ht} \%+16$

$\ln \xi=0$

Return

Procedure Exit

Edit

Return

Procedure Help

Cls

Print "This progra has two stages; the first, the .ARR file processor"

Print "requires a disk with a series of . ARP files. If there are no , ARR"

Print "files on the disk an error (two bonbs) will result."

Print "The screen during this processing is overlaid with deposits however,"

Print the screen density is not representative of the frequency at $(x, y)$."

Print "The second stage slices the cunulative histogran at the value of a"

Print "wich is input at the proupt. .SCR, .FHE, and. ARR files are then"

Print "set-up after the input whether the higher or lower slices are chosen."

Print "After viewing, these files naned (Freqhist), can be further processed"

Print "by existing uethods"

Print "If you have inserted an Array Disk and have ready an Enpty and Foratted Disk."

Print "and are ready to process . ARR files then....type ' $y$ '"

Print "When the new screen appears then ...type or sel ect '!"

Print "Cone back when you hear the tones........."

Print 'If you want to further process a Longlist............. then type ' $\psi$ '.'

Print 'When the new screen appears then type or select ' $l$ ' or ' 2 '."

Print "and follow the proupts...."

Repeat

Answer $\{=$ Inkey

Until Answer $\$=\mathbf{C H}^{\circ}$ Or Answer $\boldsymbol{s}=\mathrm{Cy}^{\prime}$

Return 
Procedure Upoer

Local Sixbitz, Exp\%, Freq\%, Fain\%, Coil $\%$, Uncoily, Itery

On Error Gosub Seg_array

Gosub Checkandload

Tax $z=$ Longlisty(16041) And 63 ! The center pixel $(200,2001$ always on.

Gosub bet_freq

Files="UPPER"+Strs(Int(F)inh/Taaxht100))+". SCR"

Cls

Print At 152,11 ; "Upper slice of"

Print At $(52,2)$; "frequency histogran"

Print At $(52,4)$; "Pixels displayed"

Print At $(52,5)$; 'represent sites with"

Print At $(52,6)$; "frequency a $>=$ "Fain $\% /$ Taxy

Print At $(52,7)$ : "based on "Toaxy' "deposits"

Print At $(52,20)$ : $^{\text {" }} \%$ of screen painted"

Order $y(0)=1$

Order $\%(1)=201$

For Iter $y=1$ To 32000

Coil $\%=(I$ ter $\%-1) 15$

Freqy=Longl ist $z$ (Iter $y$ )

If Iter\% Mod 320=0 Then

Print At $(52,20)$; Using "B(4", Iter $\$ 1100 / 32000$

Endif

If Freqtis>0 Then

For Sixbit $\%=0$ To 4

Uncoil $\%=$ Coil $y+$ Sixbity.

Expy=64:5ixbitz

Freq $h=$ Longlistz(Iter $\%$ ) And (63tExp

If Freq $2<>0$ Then

Div Freq\%, Exp\%

If Freqh) $=$ Feint Then

$x p i x e l x=$ Uneoil $1 \% \backslash 400$

Ypixel $\mathrm{b}=$ Uncoily Mod 400

Plot Xpixeiy, Ypixel\%.

Ine Ordery(0)

Opticn "U1"

Order $z(0$ der $z(0))=x_{p}$ ixel $z+1024+y_{p}$ ixel $y$

Endif

Endif

Hext Sixbity

Endif

Next Iter\%

Hiden

Print At $(52,20) ;$ Spaces (28)

Bsave Files, xbios (2), 32000

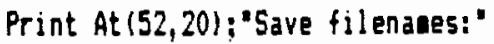

Print At $(52,21)$; Files

Print At $(52,22)$; "and"

File $\$=L$ Left (Fil es, Instr (File\$, " "1)+"ARR"

Print At $(52,23)$; Fil es

Gosub Save_segs

Return 
Procedure Lower

Local Sixbith, Expy, Freq\%, Foinz, Coil\%, Uncoily, Iter\%

On Error Gosub Seg_array

Gosub Checkandload

Tax $\%=$ Longlistz(16041) And 63 ! The center pixe! $(200,200)$ always on.

Gosub Get_freq

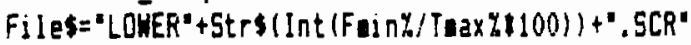

CIs

Print At $(52,1)$; "Lower slice of"

Print At $(52,2) ;$ "frequency histogras"

Print At $(52,4)$; "Pixels displayed"

Print At $(52,5)$; 'represent sites with"

Print At $(52,6)$; "frequency a $=$ "Foin\%/Teax\%.

Print At $(52,7)$ : "based on "Toaxy' "deposits"

Print At $(52,20):$ " $0 \%$ of screen painted"

Order $y(0)=1$

Ordery $(1)=20 !$

For Iter $y=1$ To 32000

Coil $y=($ Iter $y-1) 15$

Freq $\%=$ Longl ist $\%$ (Iter $\%$ )

If Iter\% Hod $320=0$ Then

Print At $(52,20):$ Using " $\$ 1 \%$ ", Iter $21100 / 32000$

Endif

If Frea\% $>0$ Then

For Sixbit $\%=0$ To 4

Uncoil $z=$ Coil $\%+5 j \times$ bity

Exp\% $=64^{\wedge}$ Sixbity

Freq\%=Longl ist $\%(I$ ter $\%$ ) And (6.3:Exp\%)

If Freax $>0$ Then

Div Freq\%, Exp\%.

If Freqk $=$ Fainh and Freq\%>0 Then

Xpixe! $y=$ Uncoil $1 \% \backslash 400$

Ypixel $\%=$ Uncoil\% hod 400

Plot xpixel $\%$, Ypixel\%.

Inc Orderylo!

Option 'UI"

Order $\%($ Order $\%(0))=x p i x$ el $\%(1024+y p i x e l \%$

Endif

Endif

Next Sixbity.

Endif

Next Itery

Hidea

Print At $(52,20)$; 5 paces $(28)$

Bsave Files, xbios(2), 32000

Print At $(52,20)$; 'Save fil enanes:"

Print At $(52,21)$; Files

Print At 152.22$)$; " and"

File\$=Left\$(Files, Instr (Files," "))+"ARR"

Print At $(52,23)$; files

Gosub Save_segs

Return 


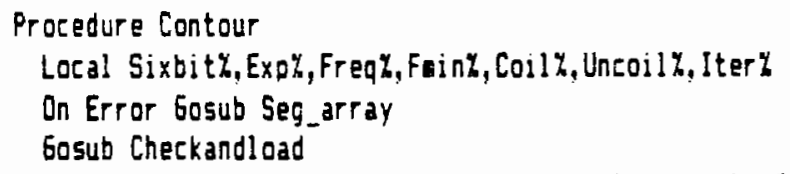

Thaxz=Longlistz(16041) And 63! The center pixel $(200,2001$ always an.

Gosub Get_freq

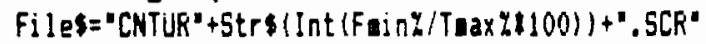

Cls

Print At $(52,1):$ "Contour slice of"

Print At $(52,2) ;$ "frequency histogran"

Print At 152,4$):$ "Pixels displayed"

Print At $(52,5)$; "represent sites with"

Print At $(52,6) ;$ "frequency a ="'Fniny/Toaxy

Print At (52,7): "based on" Thax $h^{\prime}$ "deposits"

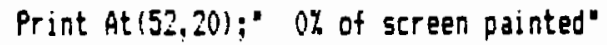

Order $\%(0)=1$

Order $\% ! 1 !=201$

For Iter $\%=1$ To 32000

Coil $y=($ Iter $y-1) 15$

Freq $y=$ Longl ist $\%$ (Iter $\%$ )

If Iter\% Mod $320=0$ Then

Print At $(52,20)$;Using "Htw", Iter $41100 / 32000$

Endif

If Freqt $<>0$ Then

For Sixbit $y=0$ To 4

Uncoil $\%=$ Coil $y+$ Sixbit $\%$

Exp ${ }^{4}=64^{\wedge}$ Sixbity

Freqy=Longlist (Itery) And (63)Exp\%)

If Freativo Then

Div Freq $\%$, Exp\%.

If Freq\%=Fainz Then

Xpixe! $y_{0}=$ Uncoil $1 \% 1400$

Ypixel\%=Uncoil\% nod 400

Plot Xpixely, Ypixel\%

Ine Ordery(0)

Option "U1"

Ordery(Ordery (0)) =xpjxel $\not 11024+Y p i x e l y$

Endif

Endif

Next Sixbity

Endif

Next Iter $\%$

Hidea

Print At $(52,20)$; Spaces (2B)

Bsave Files,xbios (2), 32000

Print At $(52,20)$; 'Save filenaes:"

Print At $(52,21)$; Files

Print At $(52,22) ; "$ and"

File $=$ Left (Files, Instr (Files," "))+"ARR"

Print At $(52,23)$; Files

Gosub Saye_segs

Return 
Procedure Checkandload

Local Fail!,Files, Devlists, Deventy

Dev]ists="ADB"

If Longlist $\%(0)=0$ Then

Do

Devent $\bar{k}=1$

Repeat

Files=Mids (Devl ist $\$$, Devent $\%, 1)+"$ : ULOMGLIST. FHG“

Print "Checking device"'Left (Files,2) "for LONELIST. FHE"

Exit If Exist (Files)

Inc Devent\%.

Until Deventy>Len (Devlists)

Exit If Devent\% $<=$ Len (Devlists)

Print "Can't find any longlist files. Flease load a disk with a"

Print "longlist at tod level and hit any key, 〈ESC〉 aborts"

Print "the progran."

Repeat

Files=Inkey

Until Files(s)"

If Fil es $=\mathrm{Chr} \$(27)$ Then

Edit

Endif

Loop

Print "Loading" 'Files

Bload Files, Lpeek (Arrptr (Longlisty(I))

Arrayfill Orderzi), 0

Endif

Return

Procedure Get_freq

Local Fuin

Print "Cutoff frequencies oust be integer oultiples of $1 /$ "isaxh;"."

Print "Frequency will autosatically be rounded to nearest $1 /$ "; Thax"; "th."

Do

Input 'Cutoff frequency (absolute $n$, or $a y)$ ';Fains

If Instr (Fuins, "ya)<>o Then

Fain $=$ Tax $210.01 \mathrm{Wal}(\mathrm{Fain})+0.5$

Else

Fain $\%=V a d$ (Fain $)+0.5$

Endif

Exit If Foinz $=$ Toax:

Print "Frequency can't exceed $100 \%$ or "Teax $z^{\prime}$ 'deposits. Please reenter." Loop

Return

Procedure Seg_array

Local Ecode\%, Seg\%, Segs

Ecodel =Err

On Error Gosub Seg_array

If Ecodezs 16 Then

On Error

Error Ecode\%

Endif

Seg $z=($ Segaent $\%(29999)+1$ 
Print At $(53,21) ;$ "Seguenting . ARR file"

Print At $(53,22)$; 'Seguent" 'Seg"

Print At $(53,23)$; "Please wait..."

If Segh)! Then

Seg $\$=$ Lefts (Files, Instr (File\$, ". ")1) 'AR'+Str (Segh)

Else

Segs=Lefts/Files, Instr (Files,".")1+"ARR"

Endif

Dec Ordery (0)

Bsave Seg\$, Lpeek (Arrptr (Drder $\$(1)$ ), Order $\%(0) 14+8$

Arrayfill Order $\%$ (1), 0

$\operatorname{Order}:(0)=2$

Order $z(1)=201$

Add Seguent $\%, 29999$

Print At (52,21); Spc (29)

Print At $(52,22) ;$ Spc $(29)$

Print At $(52,23) ;$ Spc $(29)$

Resuse

Return

Procedure Save_segs

Local Bases

Base\$=Left (Files, Instr (File\$,".')

If Segnenty $=0$ Then

Bsave Files, Lpeek (Arrptr (Order $\%(1)$ ), Order $\%(0) \$ 4+8$

Else

File $\$=$ Base $\$+$ "AF'+Str\$(Int (Segent $\% / 299999)+1)$

Bsave Fil es, Lpeek (Arrotr (Ordery(1)), Order $\%(0) 14+8$

Open "R", 11, Base\$"ARR", 4

Field 11.4 As Bufs

Lset Buf \$=hk] (Segnenth+Order\%(0))

Put 11,2

Close 11

Endif

Return

Procedure Array

Print At $(52,1) ;$ "Deposit Grand Total $=0$ "

Print At $(52,3)$; "File:"

Print At $(52,5)$; "File nuaber $=": ' 0$

Print At 152,7$):{ }^{\prime} \mathrm{N}=\mathrm{O}^{\circ}$

Print At (52.9): "Out of 0 total deposits"

Repeat

Dir '1.ARE' To "FREQHIST. DIR"

Open "I", IO, "FREQHIST. DIR"

Repeat

Gosub Loader

Gosub Process

Until Eof (10)

Repeat

Print At $(52,22)$; "Hit any key to continue"

$P=$ Trunc $(125 /$ Rnd $(1)+0.5)$

Sound $1,15,1 F, 50$

Until Inkey $\$>"$ 


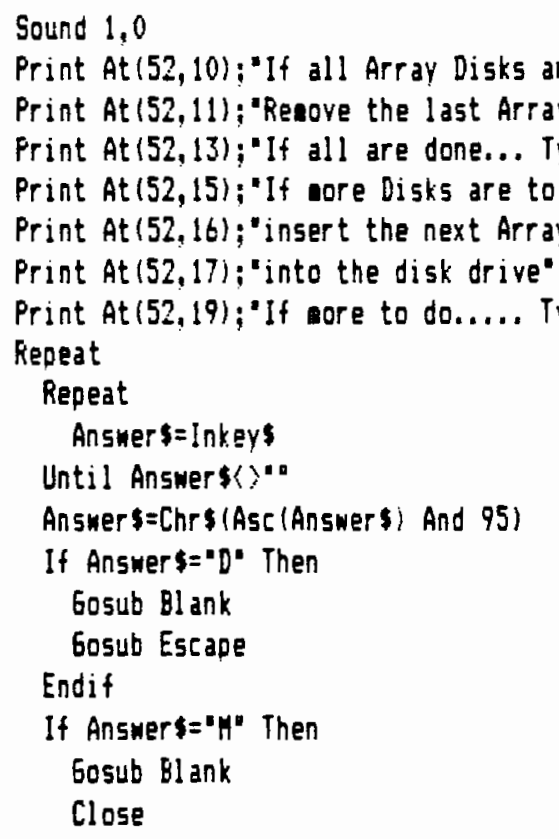


Ine 6rand\%.

If (NY-1) Mod $100=0$ Then

Print At $(72,1)$; Using 'Itwat', Grand\%

Print At $(54,7)$; Using "Amt*", N\%-1

Endif

Inc $N$ \%

Until Ny>0rder\%(0)

Print At $(52,1)$; Using "Deposit Grand Total=\$ttt\$", Gr and\%

Longlist $\%(0)=6 \mathrm{r}$ and\%

Return

Procedure Loader

Arrayfill Order $z(1,0$

Input $10, F i l e s$

Bload Files, Lpeek (Arrptr (Orderz(1))

Return

Procedure Blank

Deffill 0,1

Pbox 401,124,639,399

Return 
APPENDIX B

NUMERICAL DATA

TABLE I I

FRACTAL DIMENSION DATA FOR INDIUIDUAL SMALL AGGREGATES

Ineluding Edae

Squares 'Circles'

De

$b_{c}$

Deposit N Do

1.8355364401

1.6573104232

1.6020511402

1.6880587015

1.6609789001

1.6046445531

1.6810192676

1.6062148931

1.6785667926

1.8374010038

1.6599607926

1.7910889197

$1.652560096 ?$

1.5983715229

1.6831302681

1.8931199782

1.6661022793

1.6156322755

1.6790711059

1.6020197103

1.697172808

2.0458284656

1.658277456

1.5943751484

1.6069461333

1. 807984199

1.6621301618

1.6187794088

1.6857970137

1.6969820377

1.7187509464

1.6992186088

1.7398365792

1.6716125386

1.6162811829

1.6601788582

1.871951025

1.7310142902

1.8186270042

1.6670967371

1.6030092667

1.6769611661

1.6151354645

1.6974291693

1.6293001878

1.7139555174

1.9193907128

1.6041053153

1.6808722818

1.744598435

1.908052491

1.674623683

1.6185214248

1.7054809105

1.7050727751

1.6143191837

1.6081433871

1.688375014

1.6962384505

1.8347762916

1.774914963

1.6598486635

1.6127089791

1.6571104783

1.6045614382

1.6832531101

1.8319819071

1.6676959306

1.6104902431

1.7177188699

1.6127396026

1.7101350582

1.9340069265

1.6632756023

1.6635810306

1.6119526325

1.6881568945

1.6087703385

1.7004290546

1.6962588726

1.6186259044

1.6220593534

1.7136615252

1.6962772363
Excluding Edae

'Circles' Cireles

$D_{\text {e }}$

ع

$1.6267701358 \quad 1.6839210176$

$1.6272517401 \quad 1.6833733409$

$1.6389580136 \quad 1.6957571247$

$1.6313605475 \quad 1.688136336$

$1.641131271 \quad 1.6984915942$

1.66281653811 .7197489563

$1.6438876088 \quad 1.7012425911$

$1.6188760225 \quad 1.6749274763$

$1.6423349234 \quad 1.699262593$

$1.6575690305 \quad 1.7150032462$

$1.6253296977 \quad 1.6819975035$

$1.64673117711 \quad 1.703906851$

$1.6477016711 \quad 1.7047734201$

$1.6344183185 \quad 1.6909629697$

$1.6398140939 \quad 1.696631758$

$1.6293954963 \quad 1.6864977259$

$1.6591283631 \quad 1.7163278898$

$1.6554795156 \quad 1.7130257265$

$1.6351435214 \quad 1.6918002253$

$1.6410850401 \quad 1.69825338$

$1.6438149748 \quad 1.7007075376$

$1.6562096633 \quad 1.7132077267$

$1.6391177077 \quad 1.6958024112$ 
TABLE I I I

FRACTAL DIMENSION DATA FOR INDIVIDUAL LARGE AGGREGATES

\author{
Includino Edge
}

Squares 'Cirtes'
Excluding Edge

Squares 'Circles' Circles

$\underline{D_{e}}$

$\underline{D_{c}}$

\begin{tabular}{|c|c|c|c|c|c|c|c|}
\hline posit & $\underline{M}$ & $D_{k 0}$ & ع & ع & $D_{\varepsilon}$ & $D_{c}$ & $\underline{D_{c}}$ \\
\hline 1 & 17428 & 1.82979542 & 1.6699438252 & 1.6126899269 & 1.6747005497 & 1.6170335607 & 1.67383586665 \\
\hline 2 & 14872 & 1.8571485234 & 1.6695933746 & 1.6113824213 & 1.6750954405 & 1.6167919779 & 1.6737356634 \\
\hline 3 & $154 ! 1$ & 1.777909686 & 1.6693841613 & 1.614141633 & 1.6711044925 & 1.6163991096 & 1.6733642509 \\
\hline 4 & 15243 & 1.7746517225 & 1.6682995941 & 1.6118757841 & 1.6735022653 & 1.6164864462 & 1.67338664314 \\
\hline 5 & 12208 & 1.8329931474 & 1.66888731604 & 1.6120067388 & 1.6705759311 & 1.6138091472 & 1.6705 \\
\hline 6 & 18052 & 1.843776623 & 1.6635696705 & 1.6073511179 & 1.673057665 & 1.6160872085 & 1.67276 \\
\hline 7 & 19429 & 1.9543270665 & 1.6670483288 & 1.6106346917 & 1.6786194158 & 1.6212306642 & 1.678184297 \\
\hline 8 & 16525 & 1.7756269843 & 1.659262413 & 1.6031247851 & 1.668189111 & 1.6115455375 & 1.6682588539 \\
\hline 9 & 21268 & 1.7853591922 & 1.6672958319 & 1.6113788372 & 1.679632124 & 1.62308 & \\
\hline 10 & 17189 & 1.73540 & 1.6635703673 & 1.6088319099 & 1.6693108723 & 1.61440 & 1.671145 \\
\hline 11 & 13706 & 1.8248885544 & 1.66666992685 & 1.6092006897 & 1.6700040655 & 1.6128300932 & 1.669612847 \\
\hline 12 & 14395 & 1.73574 & 1.6680916443 & 1.610045142 & 1.671178 & 1.6131 & 1.069 \\
\hline 13 & 15720 & 1.788791 & 1.6776510535 & 1.62072031 & 1.6798353916 & 1.62209 & 1.67893644 \\
\hline 14 & 12854 & 1.8613387844 & 1.6675974528 & $1.6: 25763577$ & 1.6715090894 & 1.61675 & 1.67349765 \\
\hline 15 & 14897 & 1.73335 & 1.66498 & 1.6076303434 & 1.671864 & 1.6 & 1.67077 \\
\hline 16 & 16326 & 1.86938 & 1.660715 & 1.6052765298 & 1.664775 & 1.60896 & 1.665931117 \\
\hline 17 & $14 \div 44$ & 1.7978242181 & 1.6689430768 & 1.6111271969 & 1.6716337209 & 1.61362 & 1.6705724942 \\
\hline 18 & 16538 & 1.8051414443 & 1.6639543543 & 1.60817652 & 1.66677147 & 1.61102 & 1.66790 \\
\hline 19 & 15752 & $1.769 ? 621464$ & 1.6720236587 & 1.6144379524 & 1.675944359 & 1.61788 & 1.675013293 \\
\hline 20 & 17715 & 1.8009581388 & 1.6613071914 & 1,6057271884 & 1.66629000664 & 1.61054 & 1.667241696 \\
\hline 21 & 19255 & 1.8781901382 & 1.6670620771 & 1.6142472474 & 1.6752969272 & 1.62188 & 1.678824 \\
\hline 22 & 12613 & 1.732079330 & 1.6710463412 & 1.6159165965 & 1.6745009834 & 1.619317032 & 1.676198892 \\
\hline 23 & 16464 & 1.7898308771 & 1.67466882685 & 1.6178770816 & 1.6815031254 & 1.6243320708 & 1.68119243 \\
\hline 24 & 17161 & 1.7829201003 & 1.6688024005 & 1.6152812764 & 1.6774442037 & 1.627302901 & 1.680249824 \\
\hline 25 & 16907 & 1.8066831114 & 1.67335685334 & 1.6161727463 & 1.6796282729 & 1.6220518204 & 1.6788707044 \\
\hline 26 & 19615 & $\therefore 8434988508$ & 1.661437214 & 1.6072074545 & 1.6695702278 & 1.61454239 & 1.671326523 \\
\hline 27 & 19056 & 1.7922802635 & 1.6761666367 & 1.6198860929 & 1.6857029235 & 1.6287472478 & 1.68570740 \\
\hline 28 & 17401 & 1.8293299347 & 1.6572971729 & 1.6042628967 & 1.663857593 & 1.6101775892 & 1.66664987 \\
\hline 29 & 15680 & 1.7937369861 & 1.6629574201 & 1.6063976033 & 1.6672379819 & 1.6101002107 & 1.666882021 \\
\hline 30 & 14909 & 1.8046050822 & 1.6536853066 & 1.59716666179 & 1.6573053395 & 1.6004981638 & 1.65733531 \\
\hline
\end{tabular}

The following graphs of the radius of gyration dependence on the number of deposits are based on the radius of gyration which was calculated from the lattice origin. The slopes are also listed in Table III. 


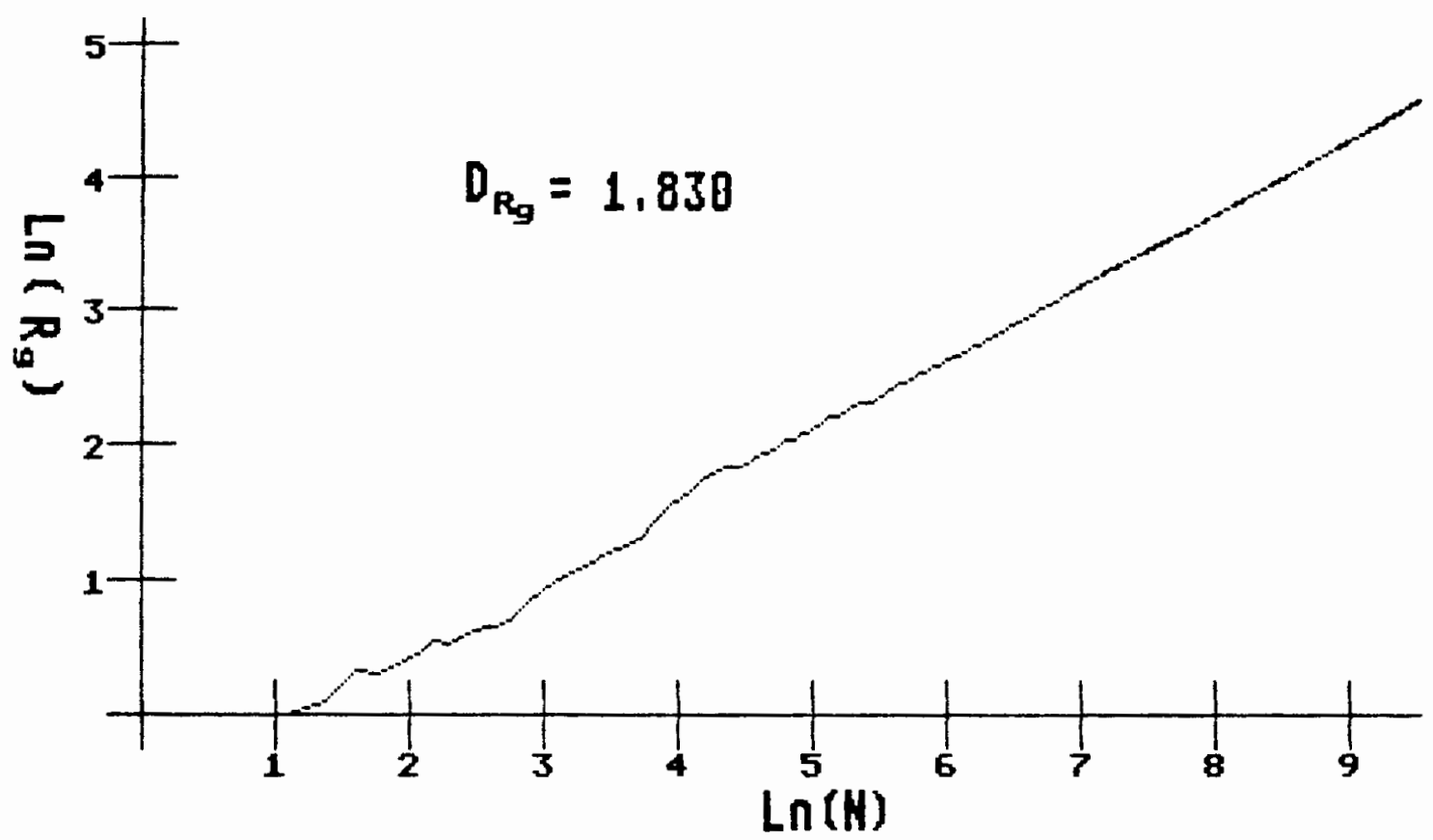

Fiqure 21. Ln(Re) vs. Ln(N) for aggregate number 1.

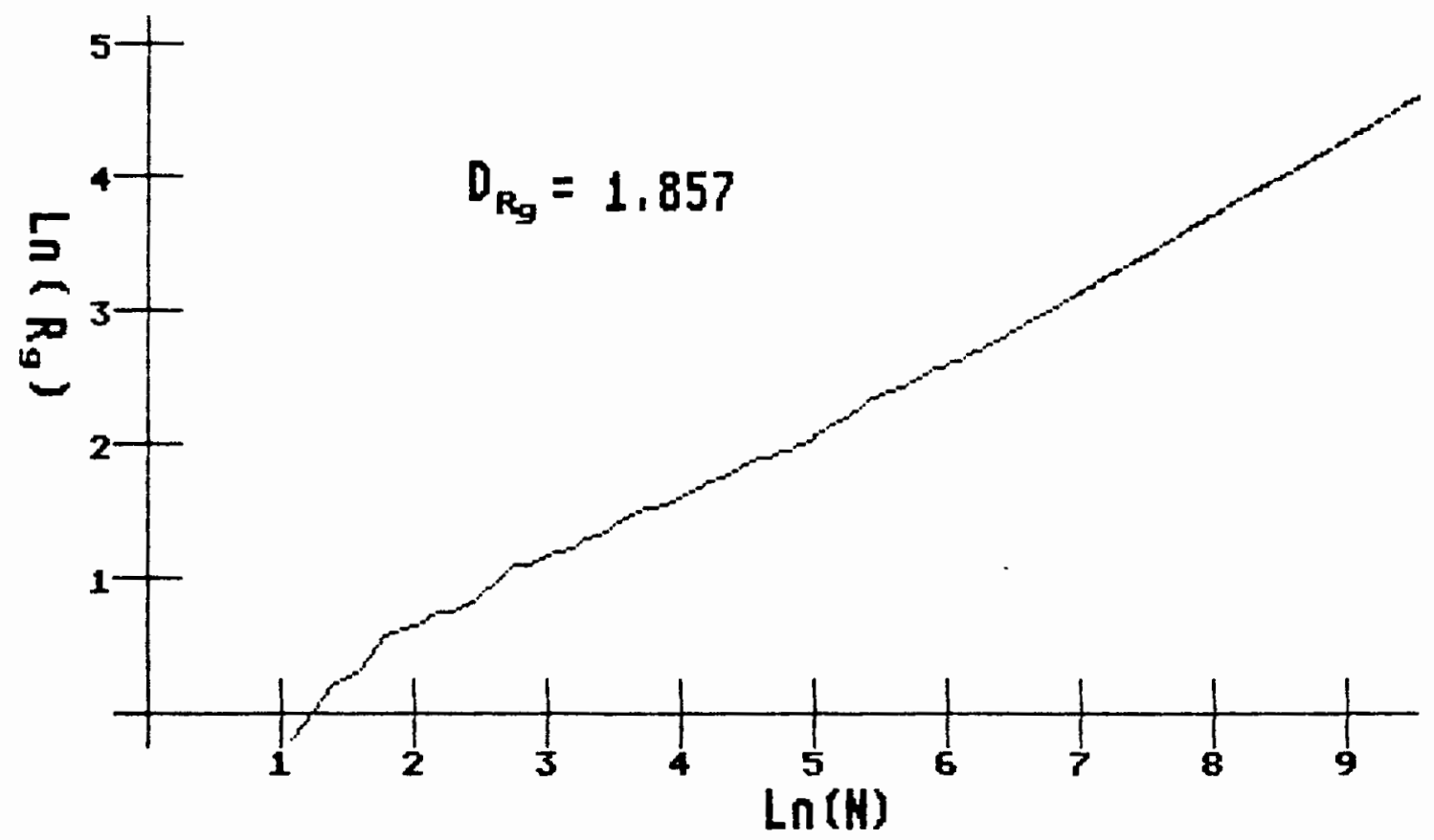

Figure 22. $\operatorname{Ln}\left(R_{0}\right)$ vs. $\operatorname{Ln}(N)$ for aggregate number 2. 


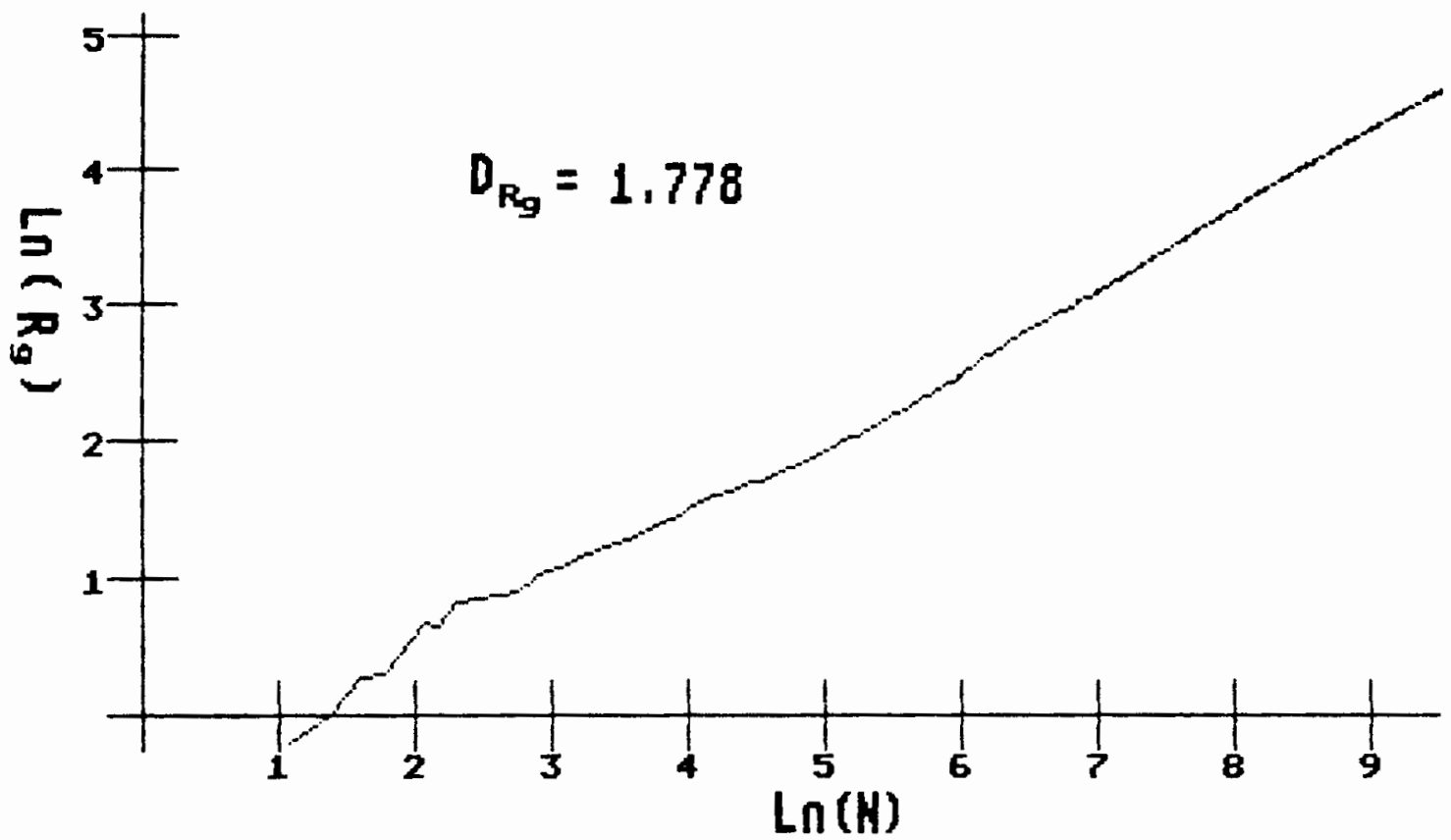

Fiqure 23. Ln(Ro) vs. Ln(N) for aggregate number 3.

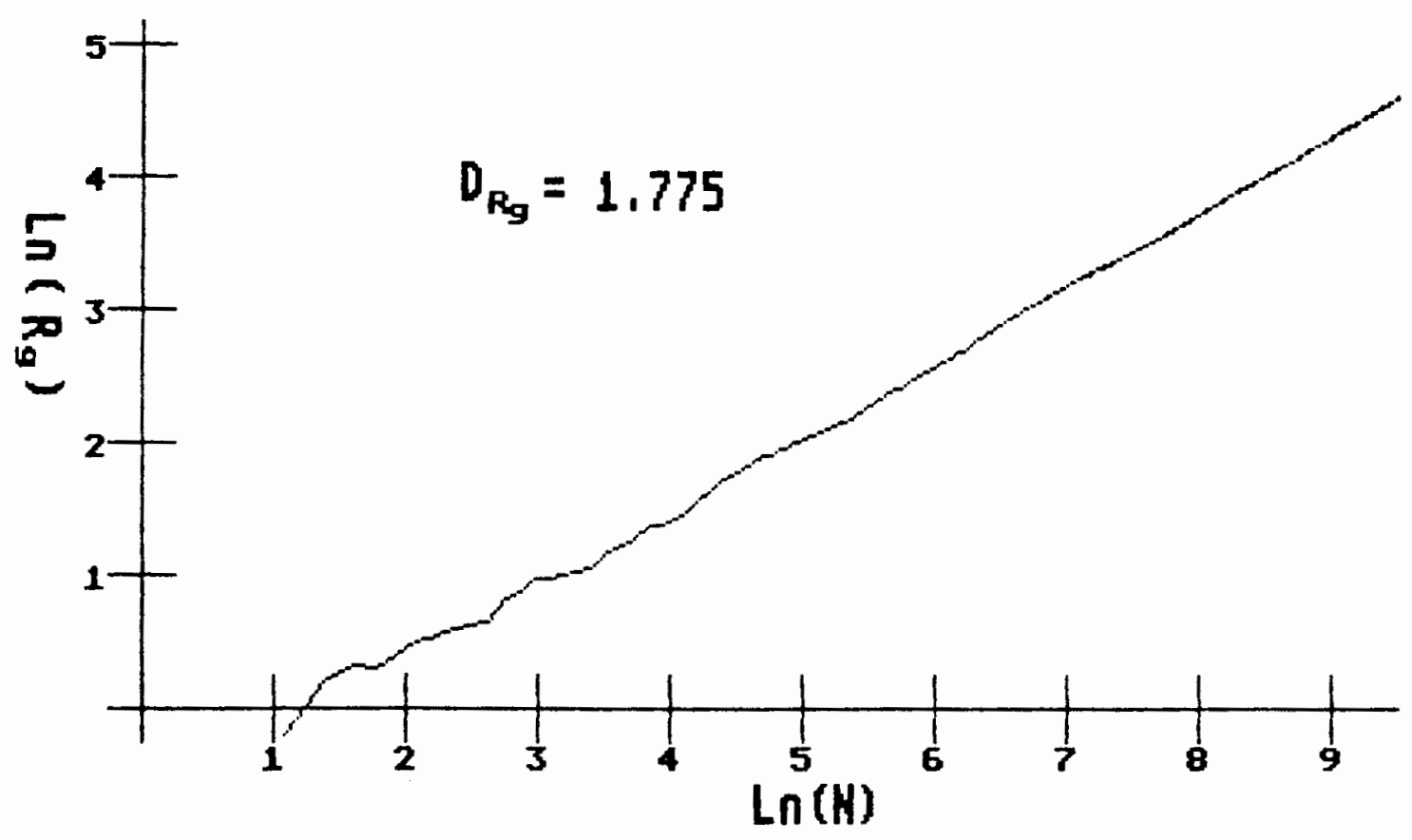

Fiqure 24. Ln $\left(R_{0}\right)$ vs. Ln(N) for aggregate number 4. 


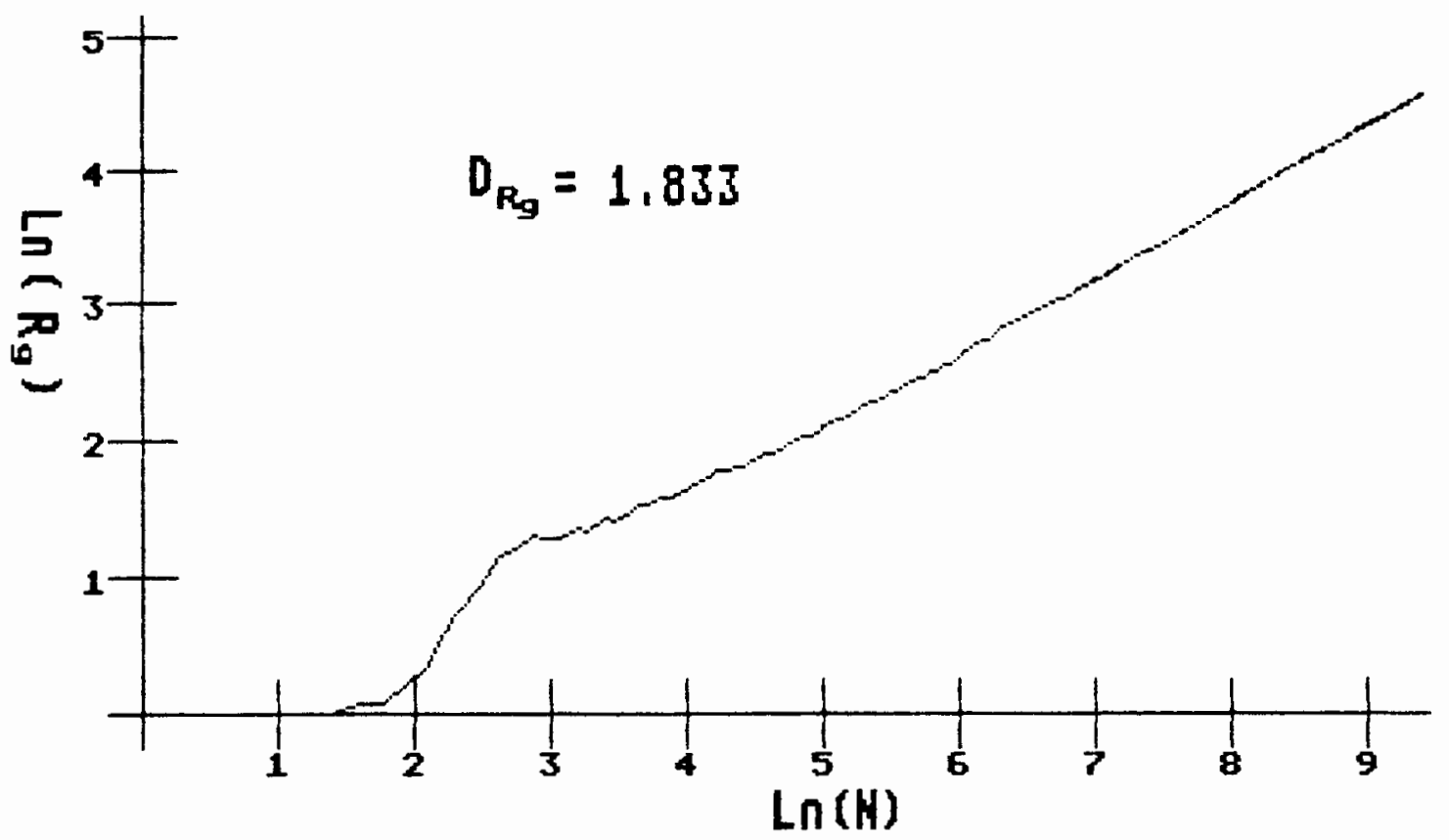

Fiqure 25. Ln $\left(R_{0}\right)$ v5. Ln (N) for aggregate number 5.

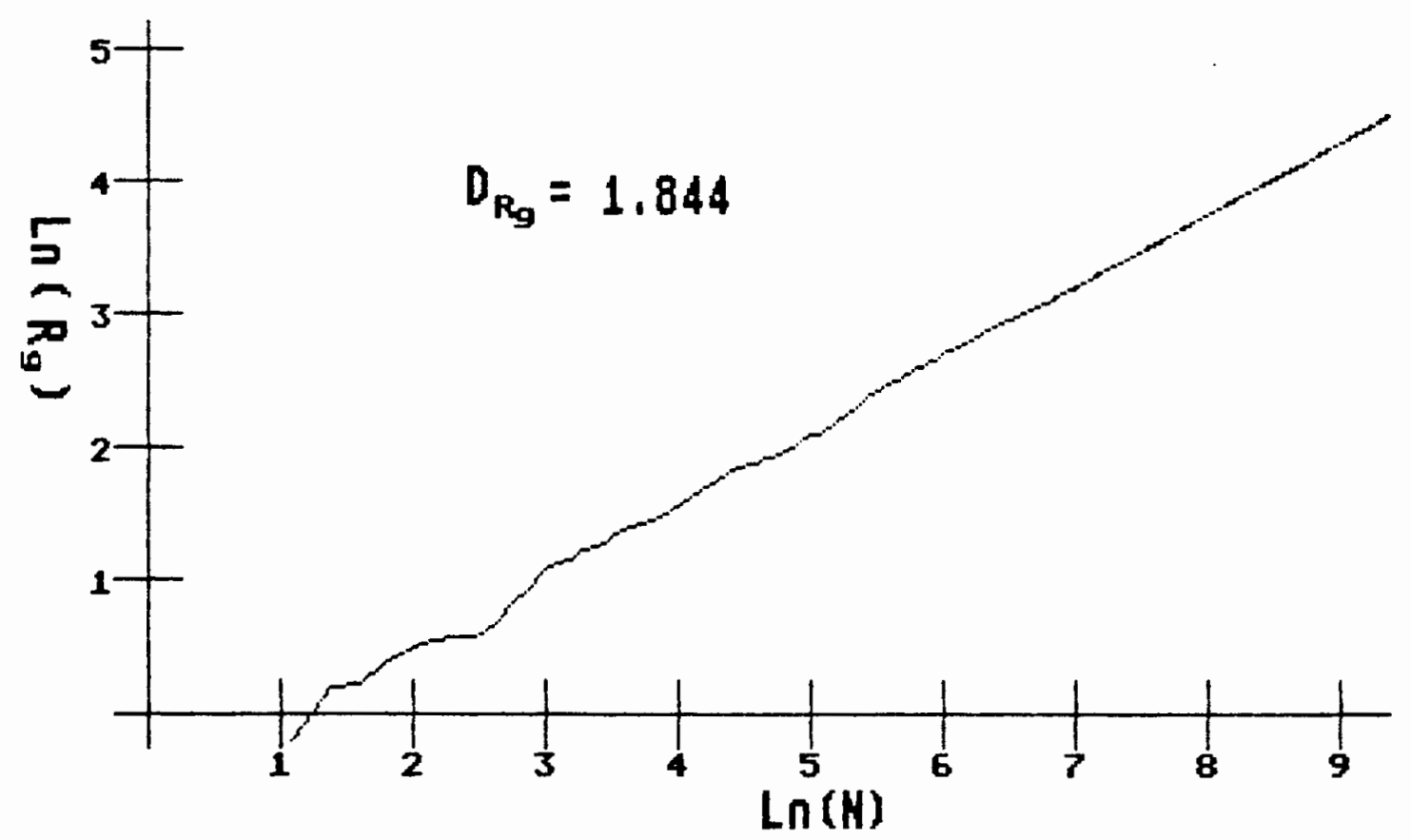

Fiqure 26. $\operatorname{Ln}\left(R_{0}\right)$ vs. $\operatorname{Ln}(N)$ for aggregate number 6. 


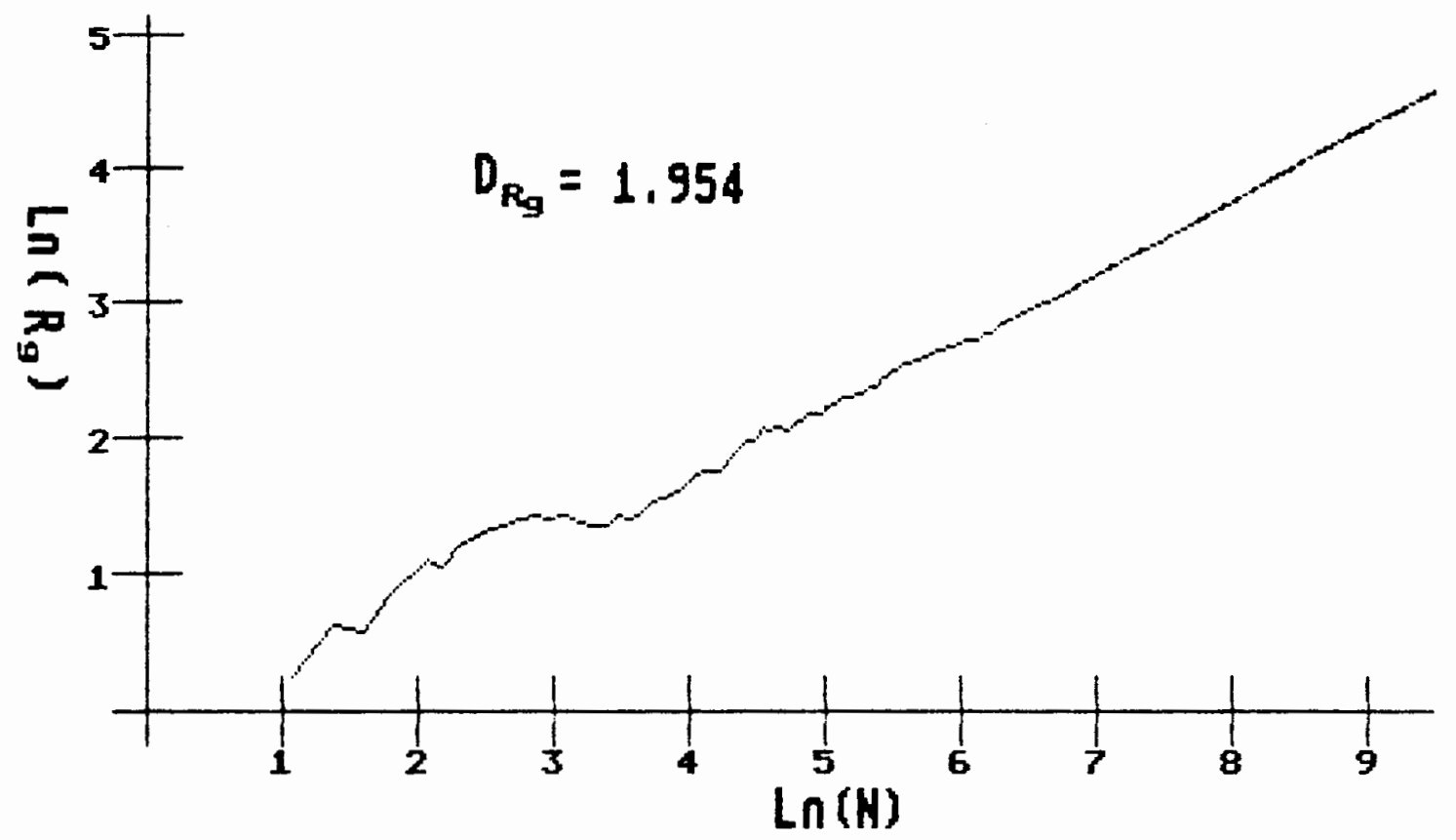

Figure 27. Ln $\left(F_{\infty}\right)$ vs. Ln(N) for aggregate number 7.

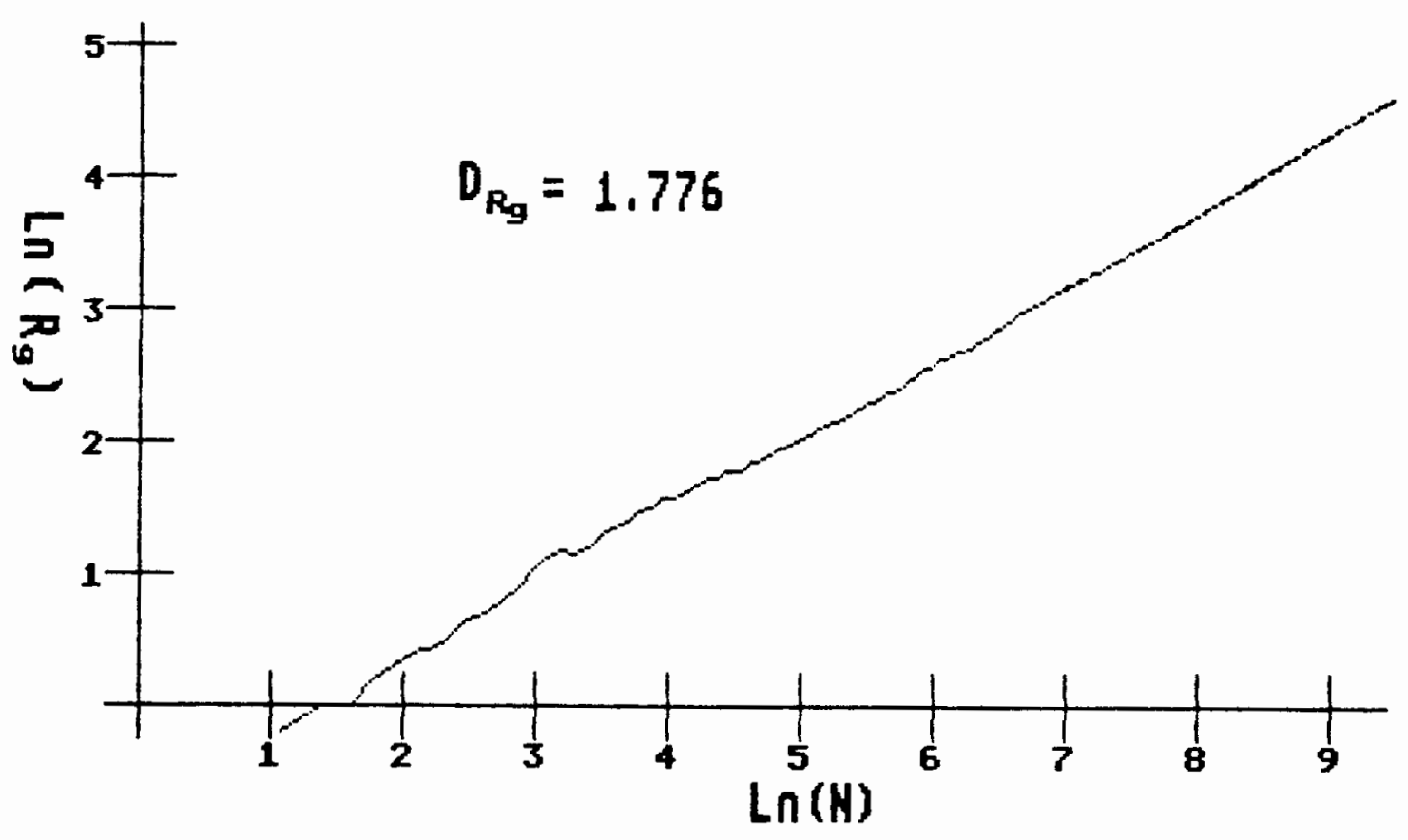

Fiqure 28. Ln $\left(R_{0}\right)$ vs. $\operatorname{Ln}(N)$ for aggregate number 8. 


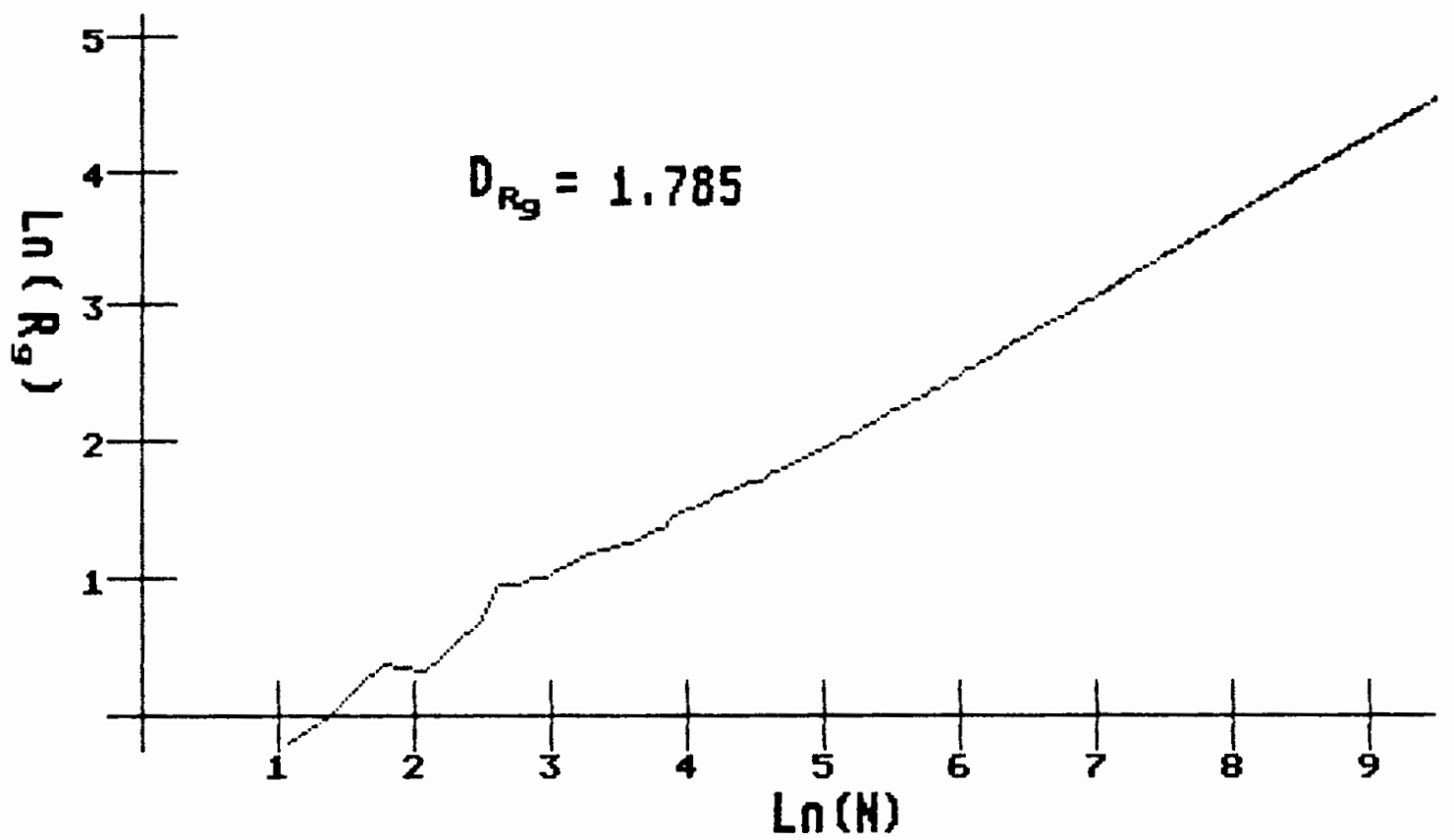

Figure 29. $\operatorname{Ln}\left(R_{0}\right)$ vs. $L n(N)$ for aggregate number 9.

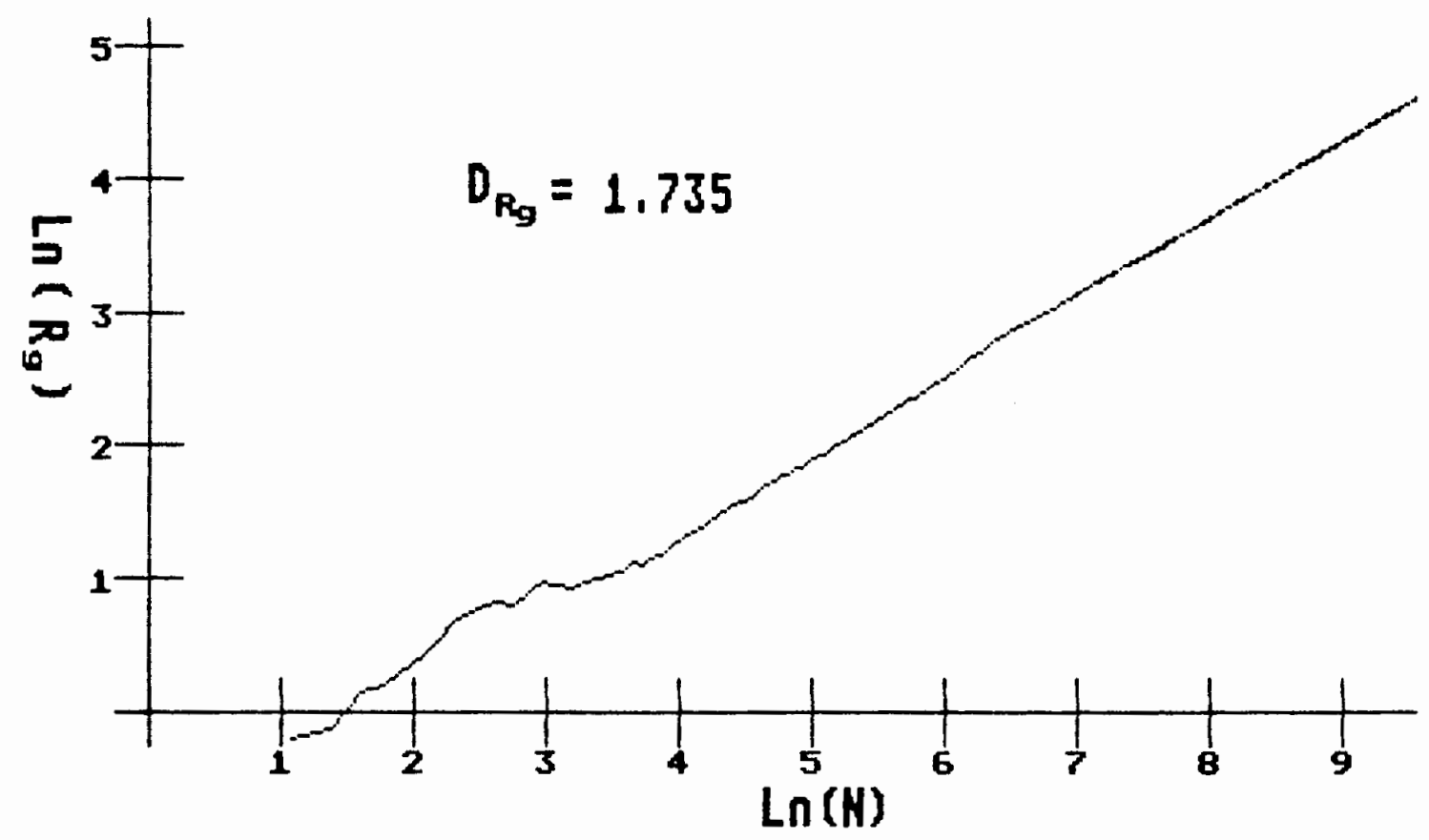

Fiqure 30. Ln(Ro) vs. Ln (N) for aggregate number 10. 


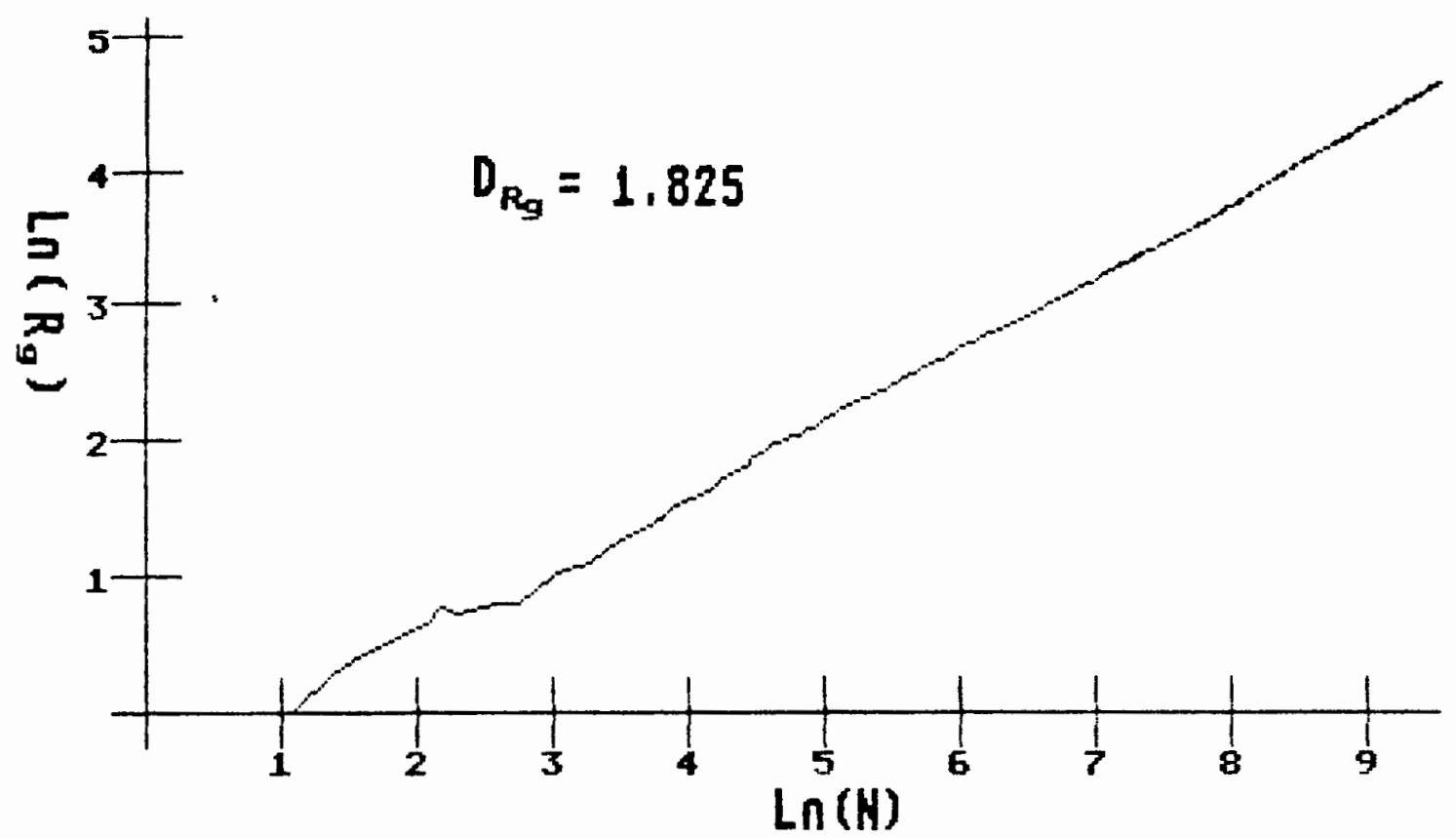

Fiqure 31. $\operatorname{Ln}\left(\mathrm{F}_{0}\right)$ vs. $\operatorname{Ln}(N)$ for aggregate number 11.

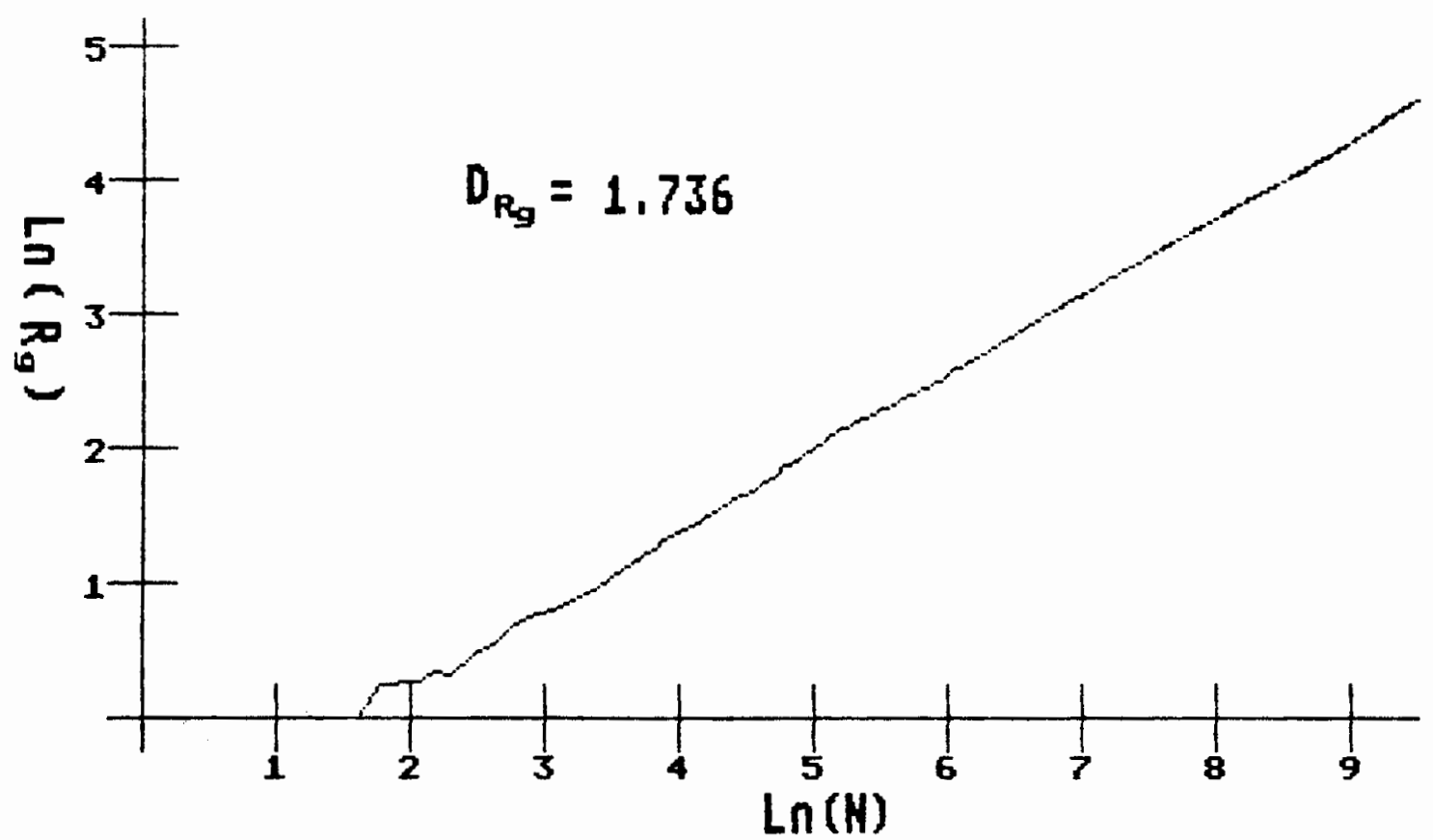

Fiqure 32. $\operatorname{Ln}\left(R_{0}\right)$ vs. $\operatorname{Ln}(N)$ for aggregate number 12. 


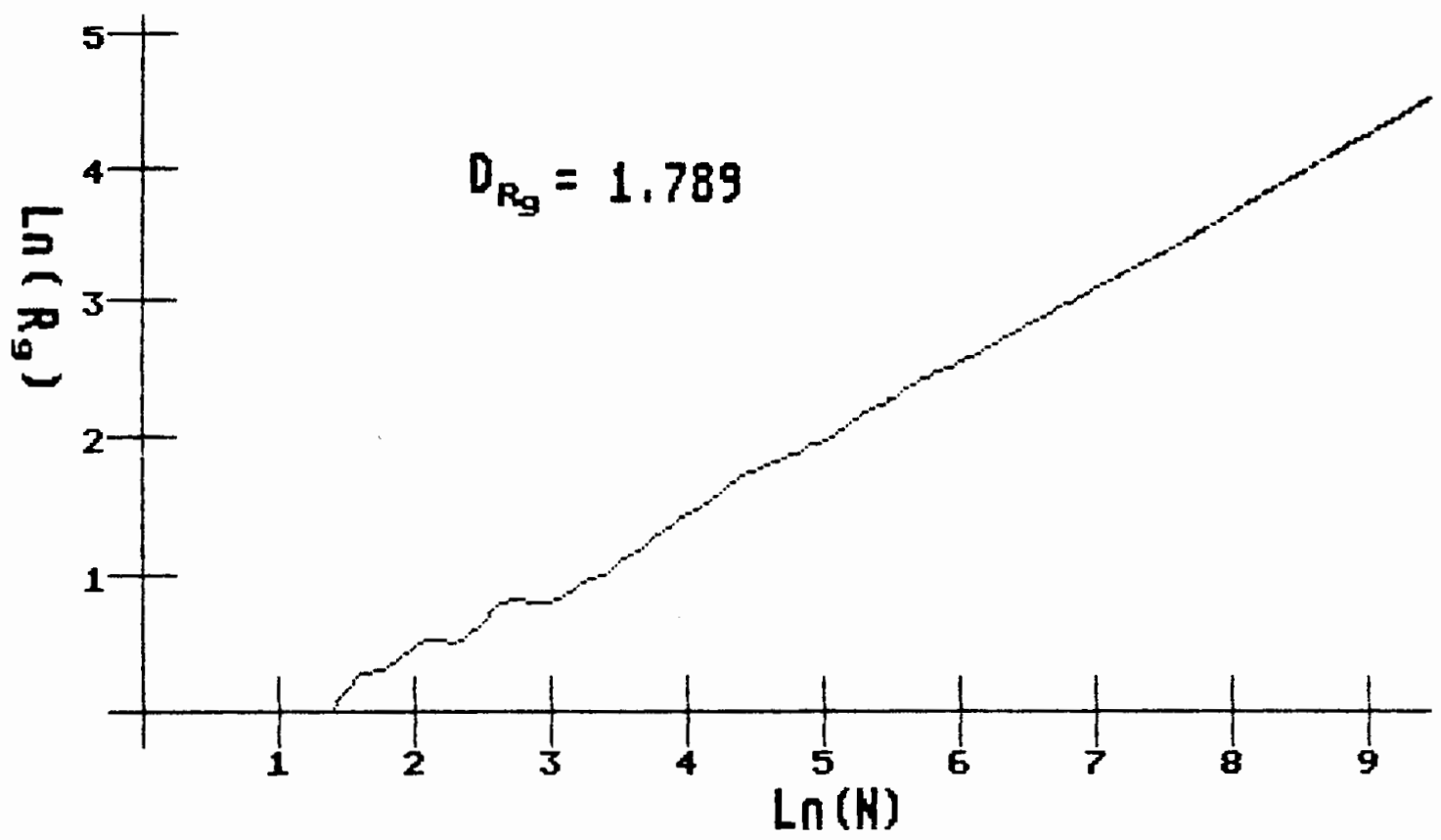

Fiqure 3.3. $\operatorname{Ln}\left(R_{0}\right)$ vs. $L n(N)$ for aggregate number 13.

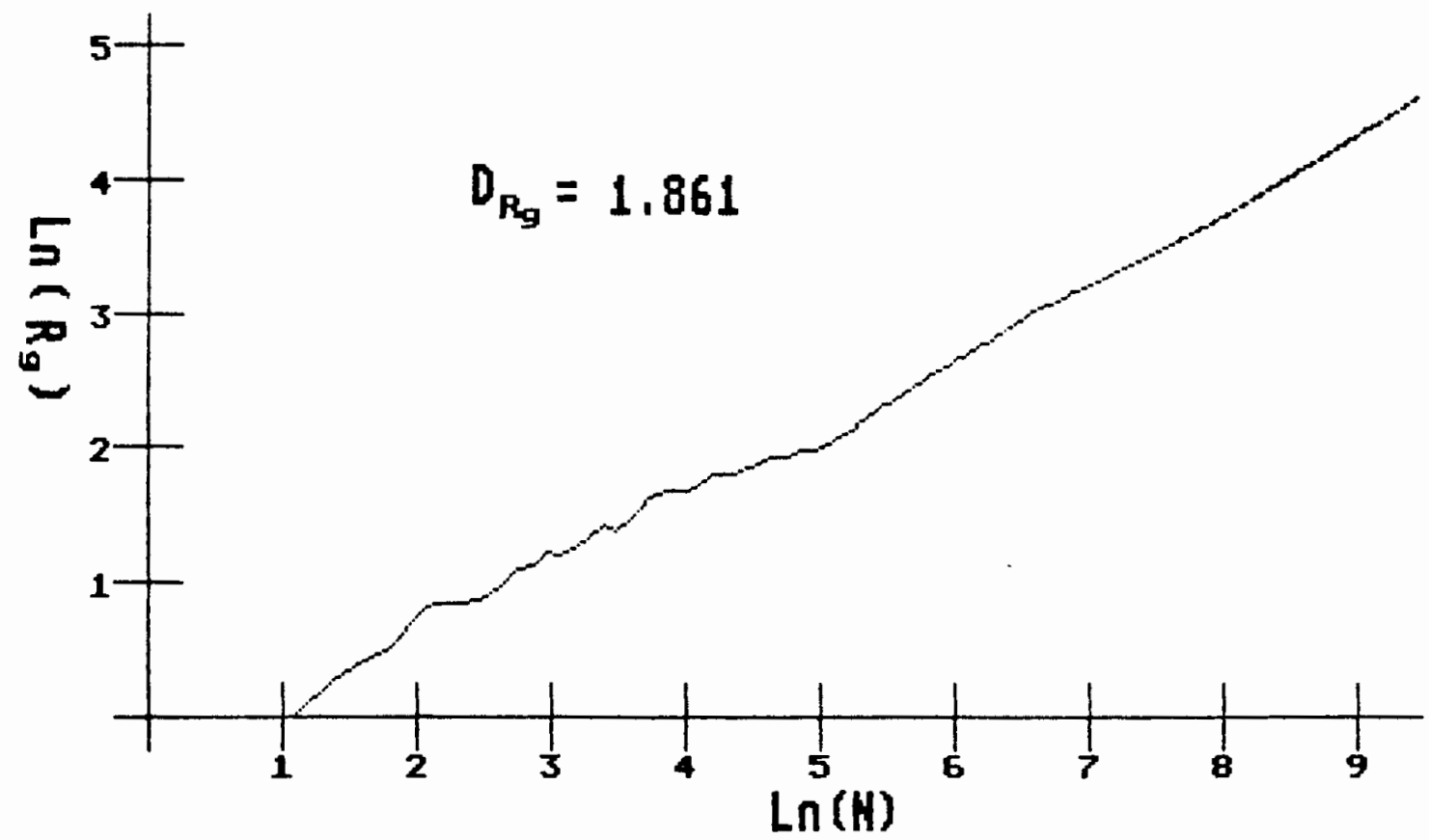

Figure 34. Ln(Ro) vs. Ln (N) for aggregate number 14. 


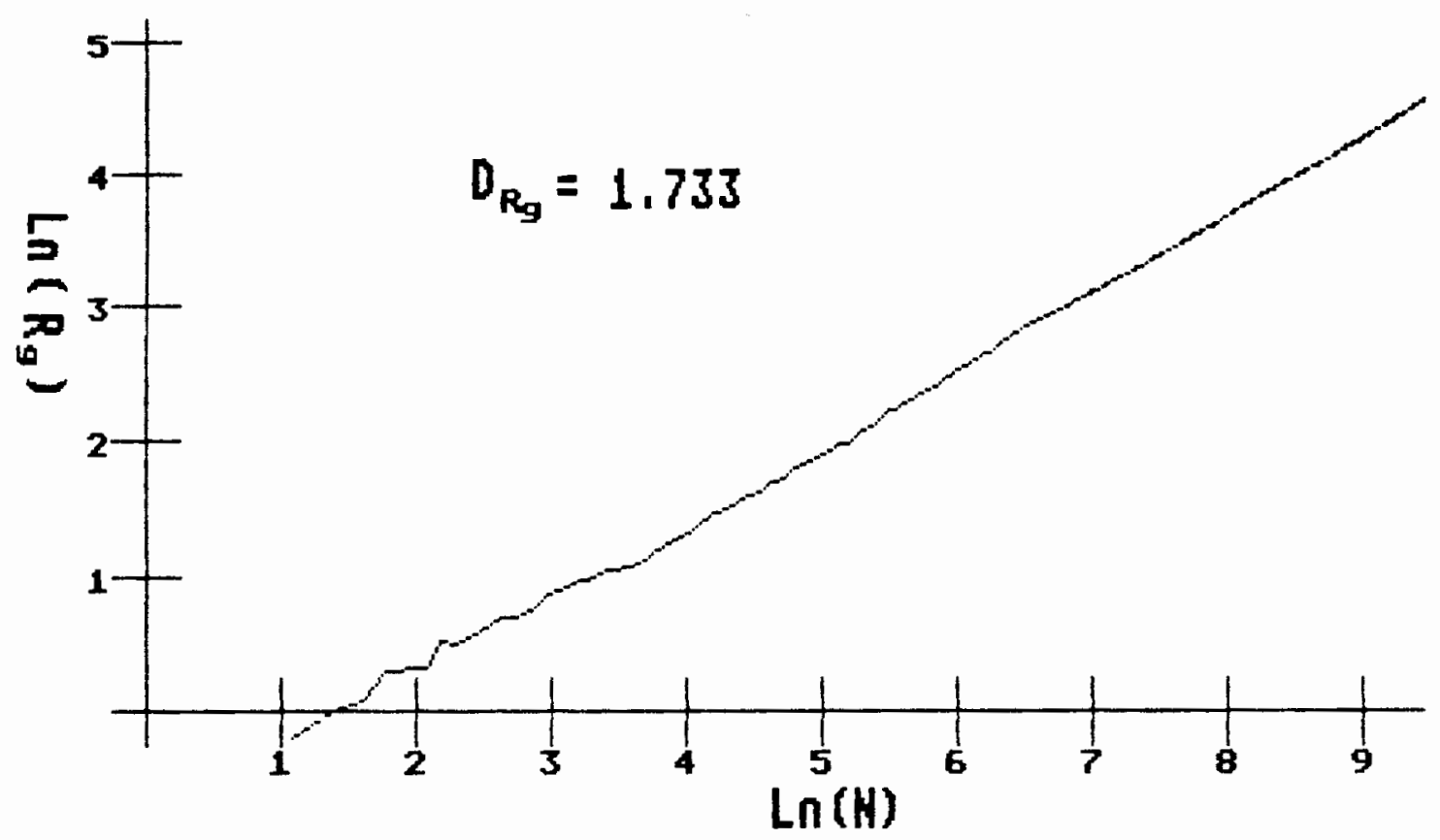

Fiqure 35. Ln $\left(R_{0}\right)$ vs. Ln $(N)$ for aggregate number 15.

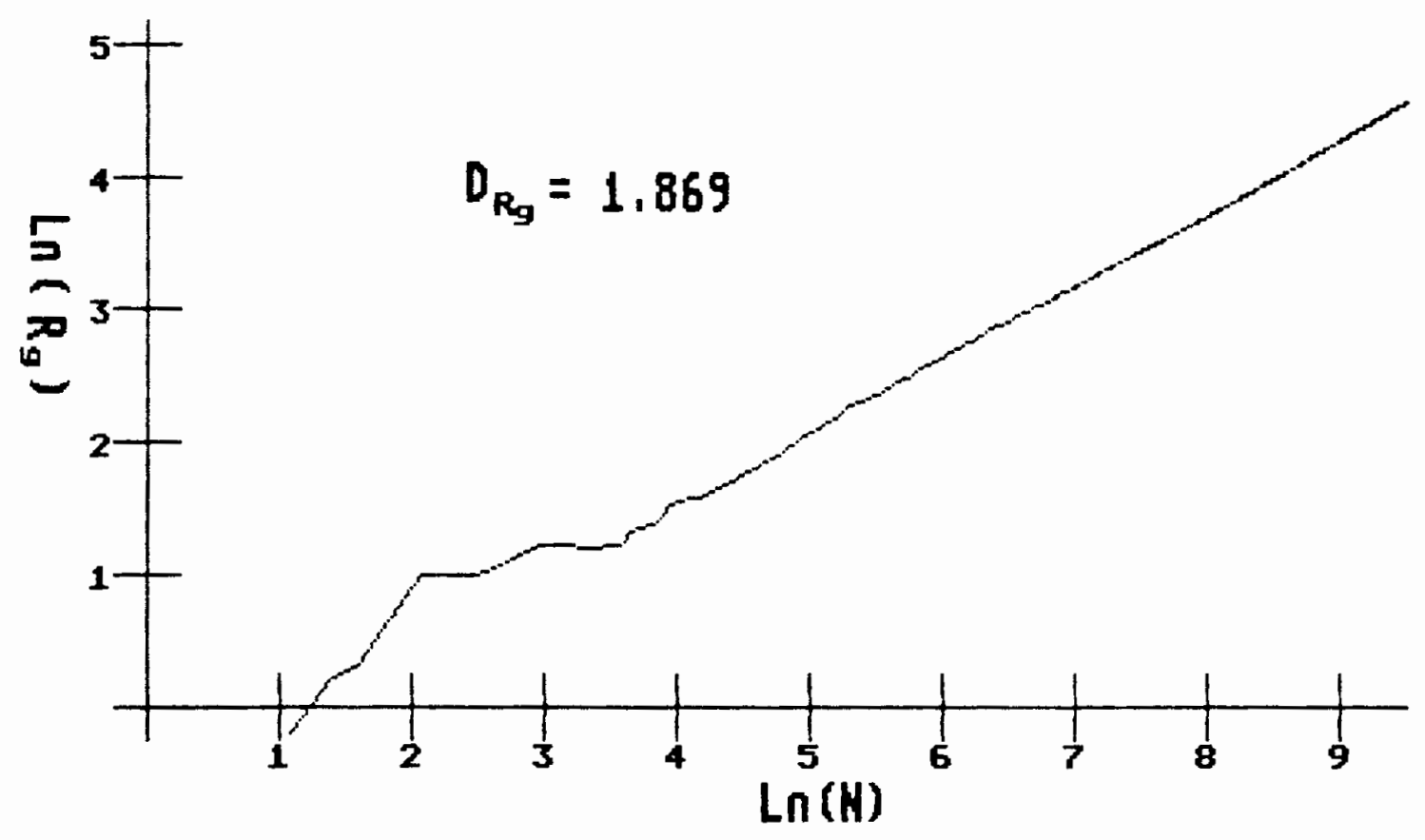

Fiqure 36. $\operatorname{Ln}\left(R_{0}\right)$ v5. $L n(N)$ for aggregate number 16. 
130

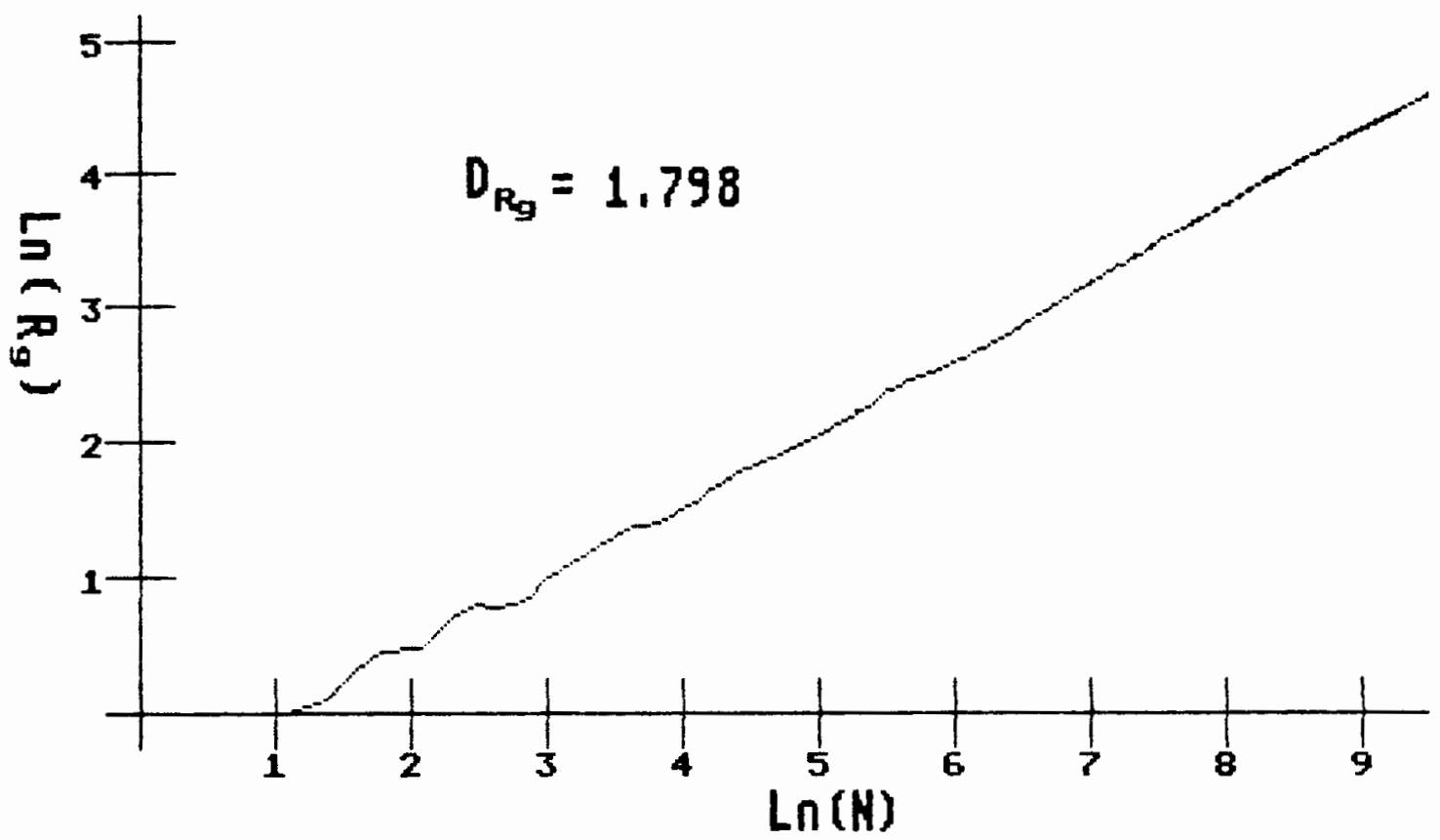

Fiqure 37. Ln(Ro) vs. Ln(N) for aggregate number 17.

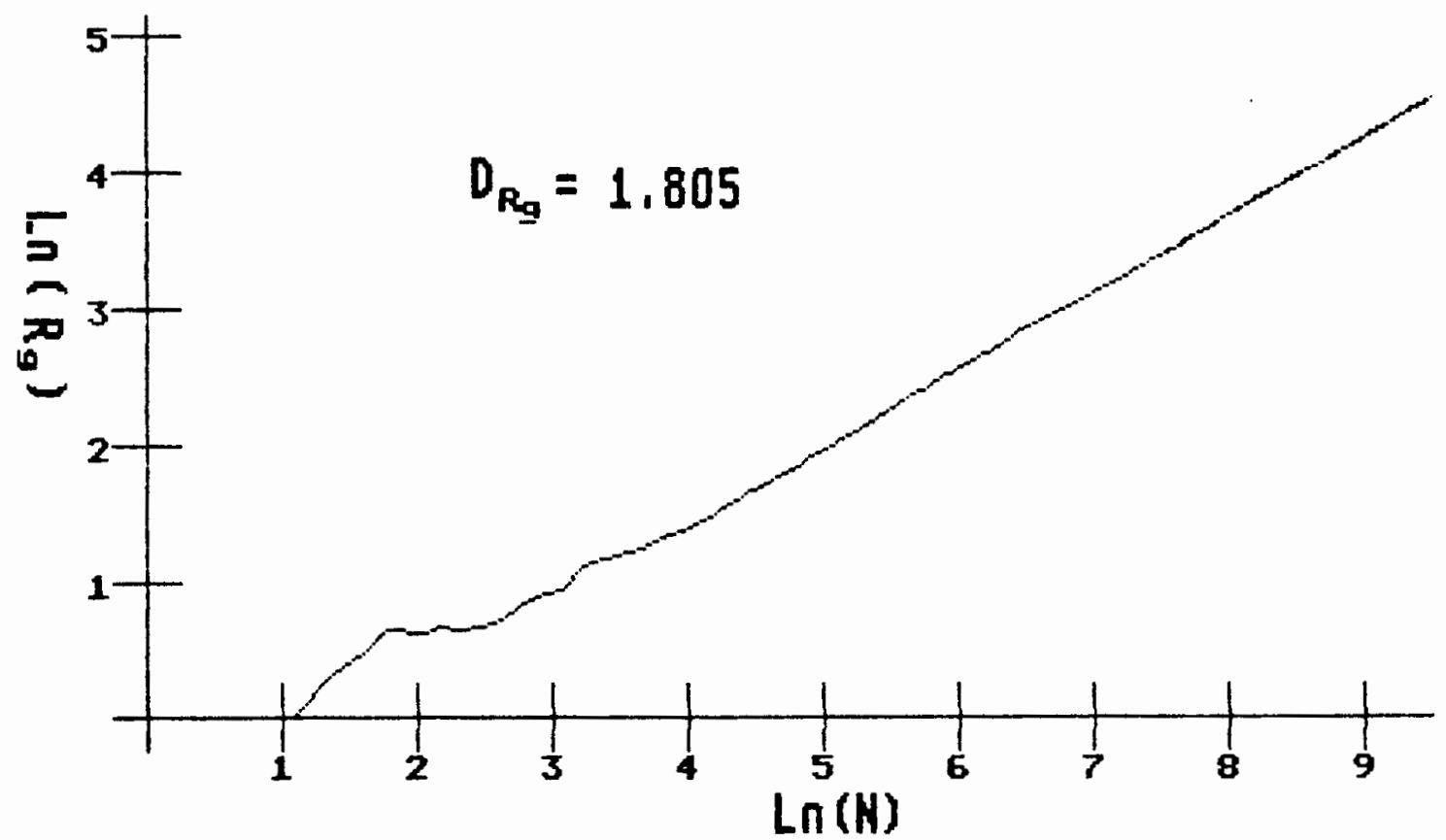

Figure 3B. Ln $\left(R_{0}\right)$ v5. Ln (N) for aggregate number 18. 


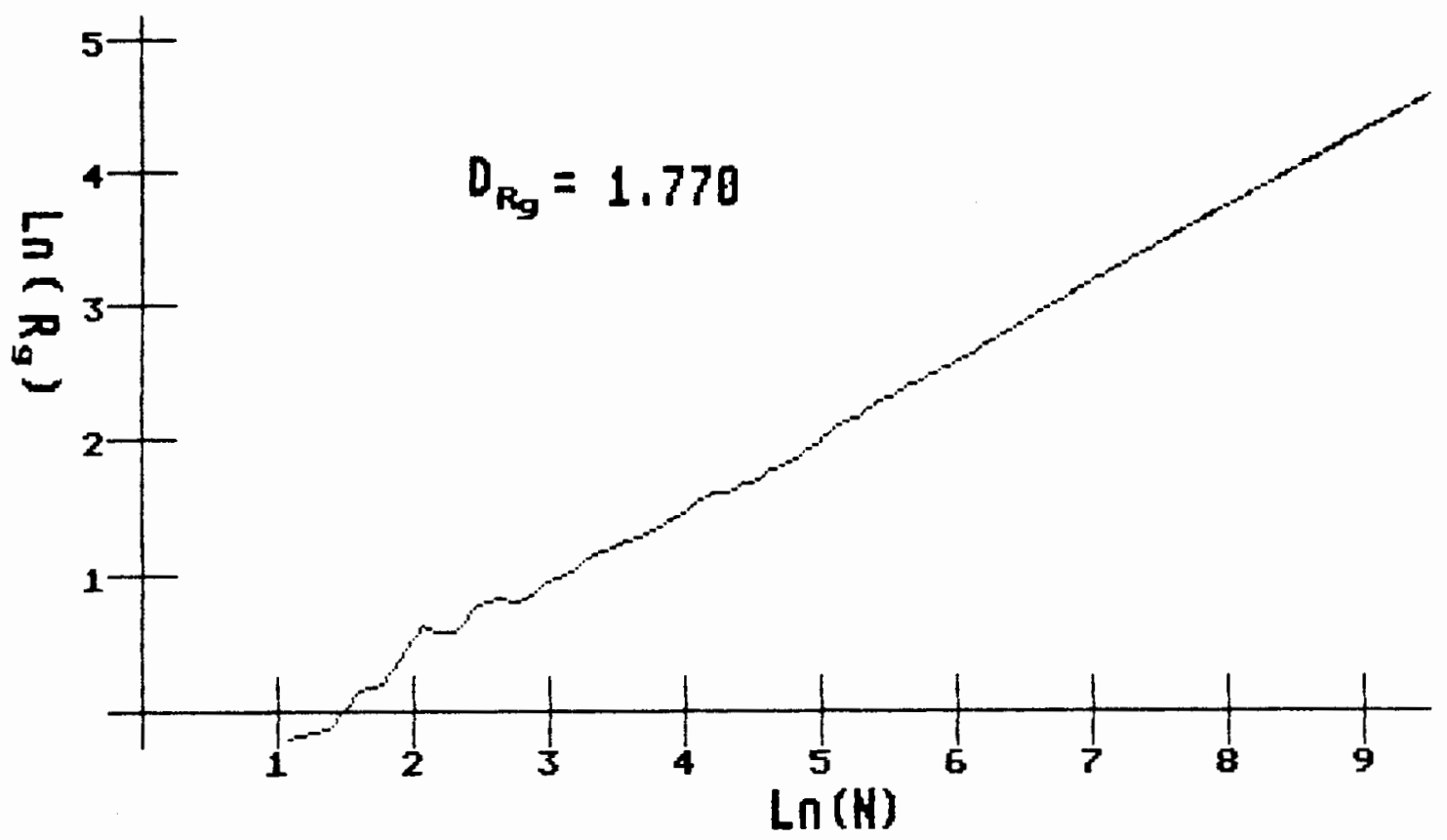

Figure 39. $\operatorname{Ln}\left(R_{0}\right)$ vs. Ln (N) for aggregate number 19.

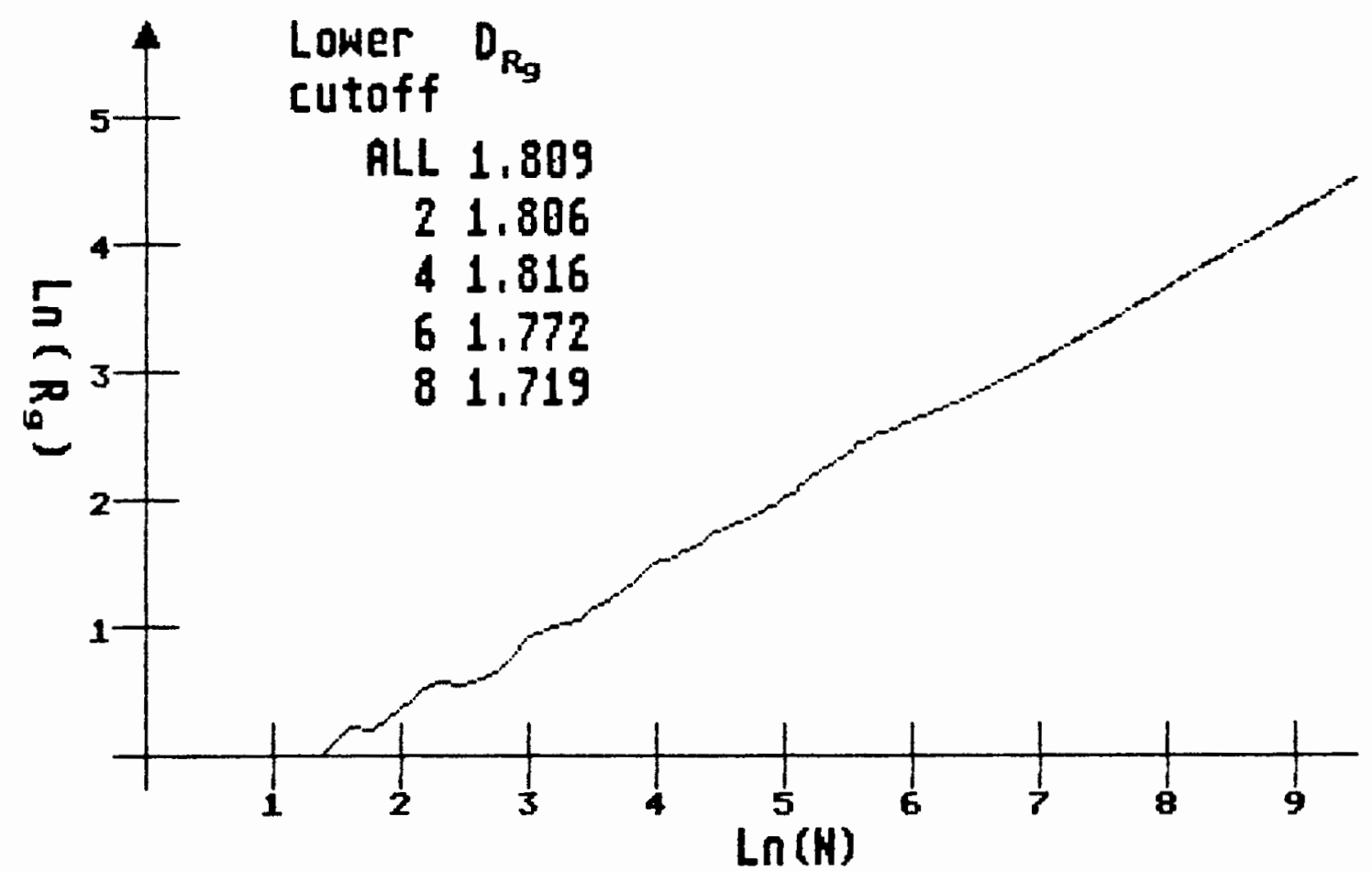

Fiqure 40. Ln $\left(R_{\odot}\right)$ vs. Ln (N) for aggregate number 20. 


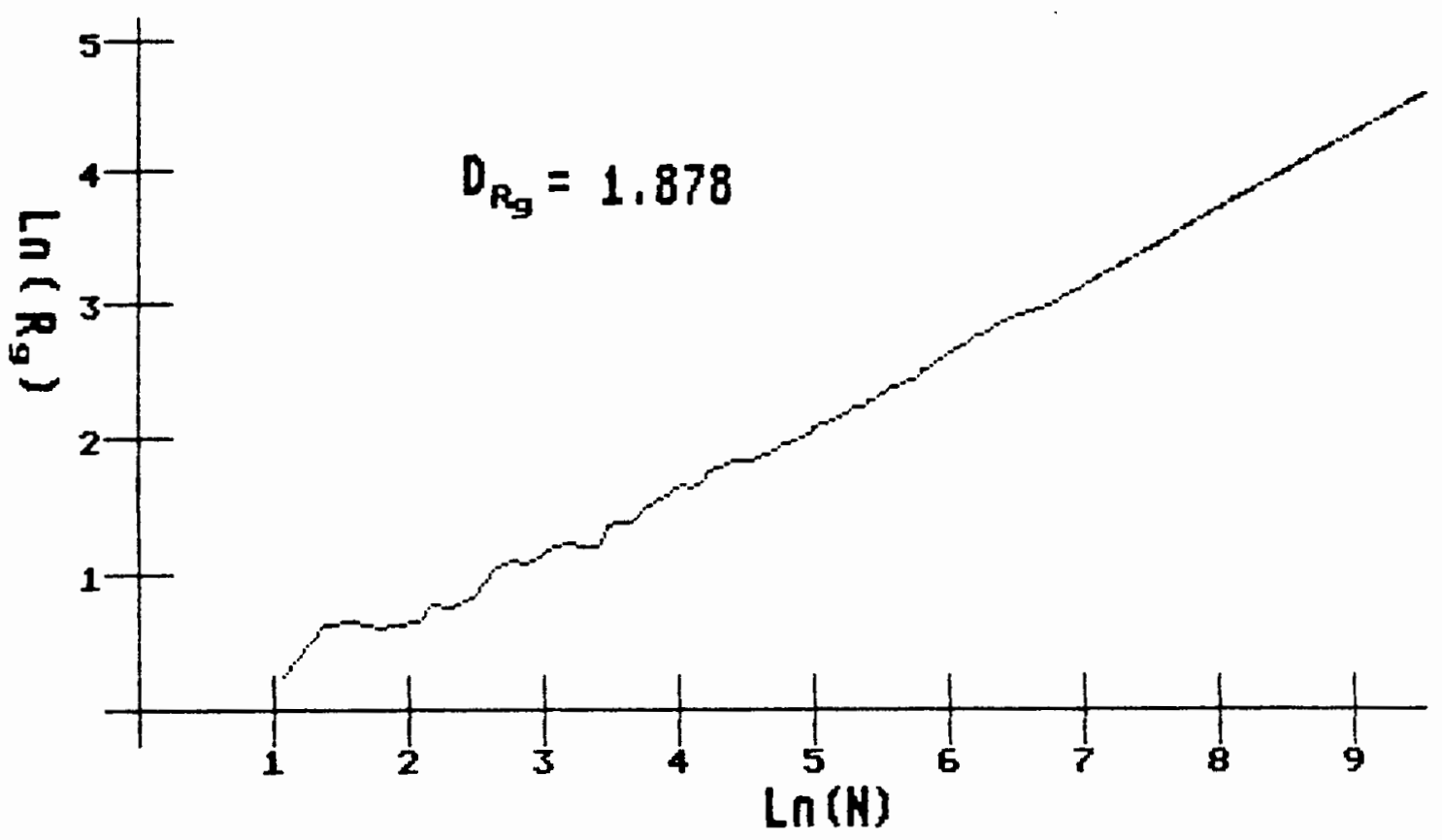

Figure 41. Ln $\left(R_{0}\right)$ vs. Ln (N) for aggregate number 21 .

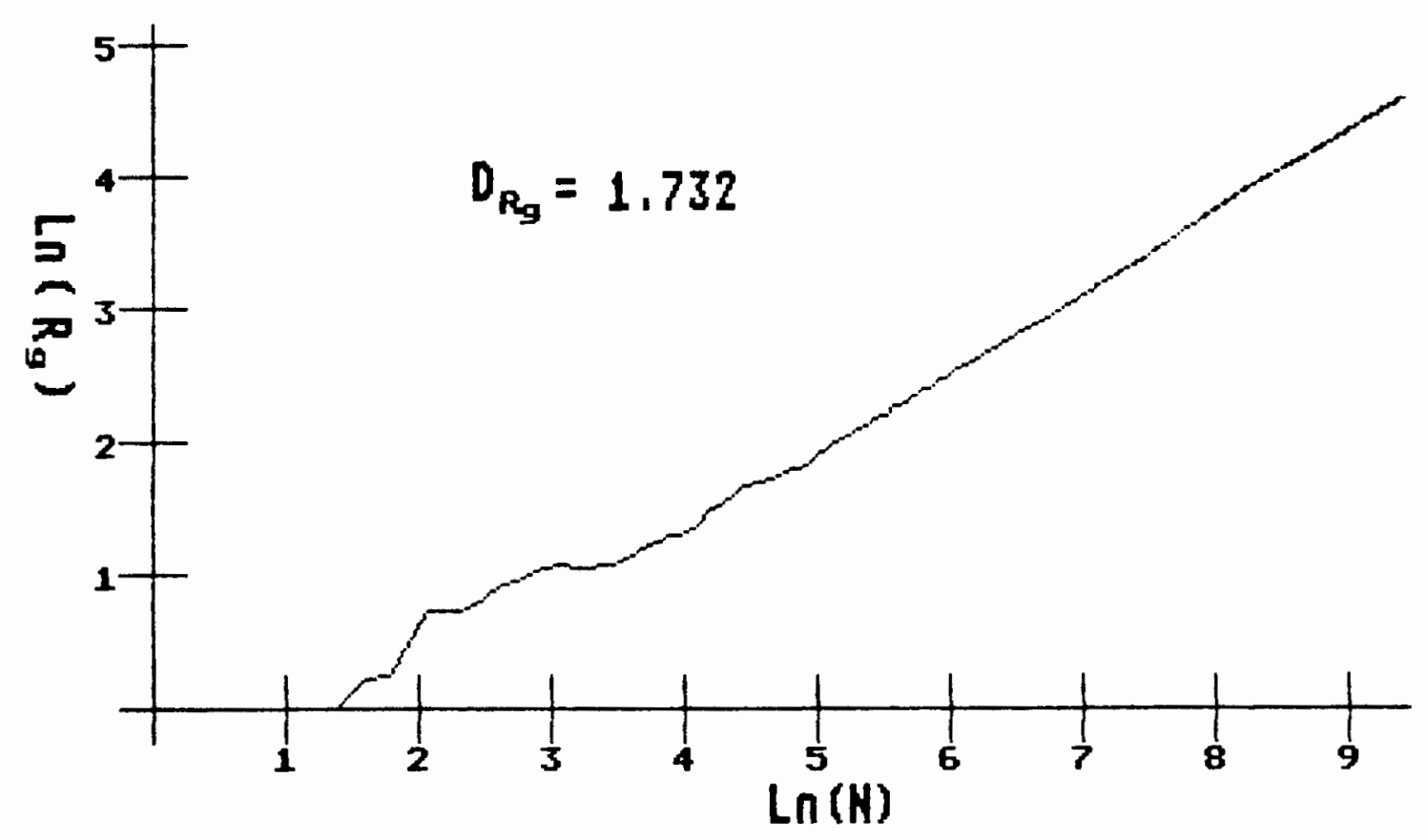

Fiqure 42. Ln $\left(R_{0}\right)$ vs. Ln (N) for aggregate number 22. 


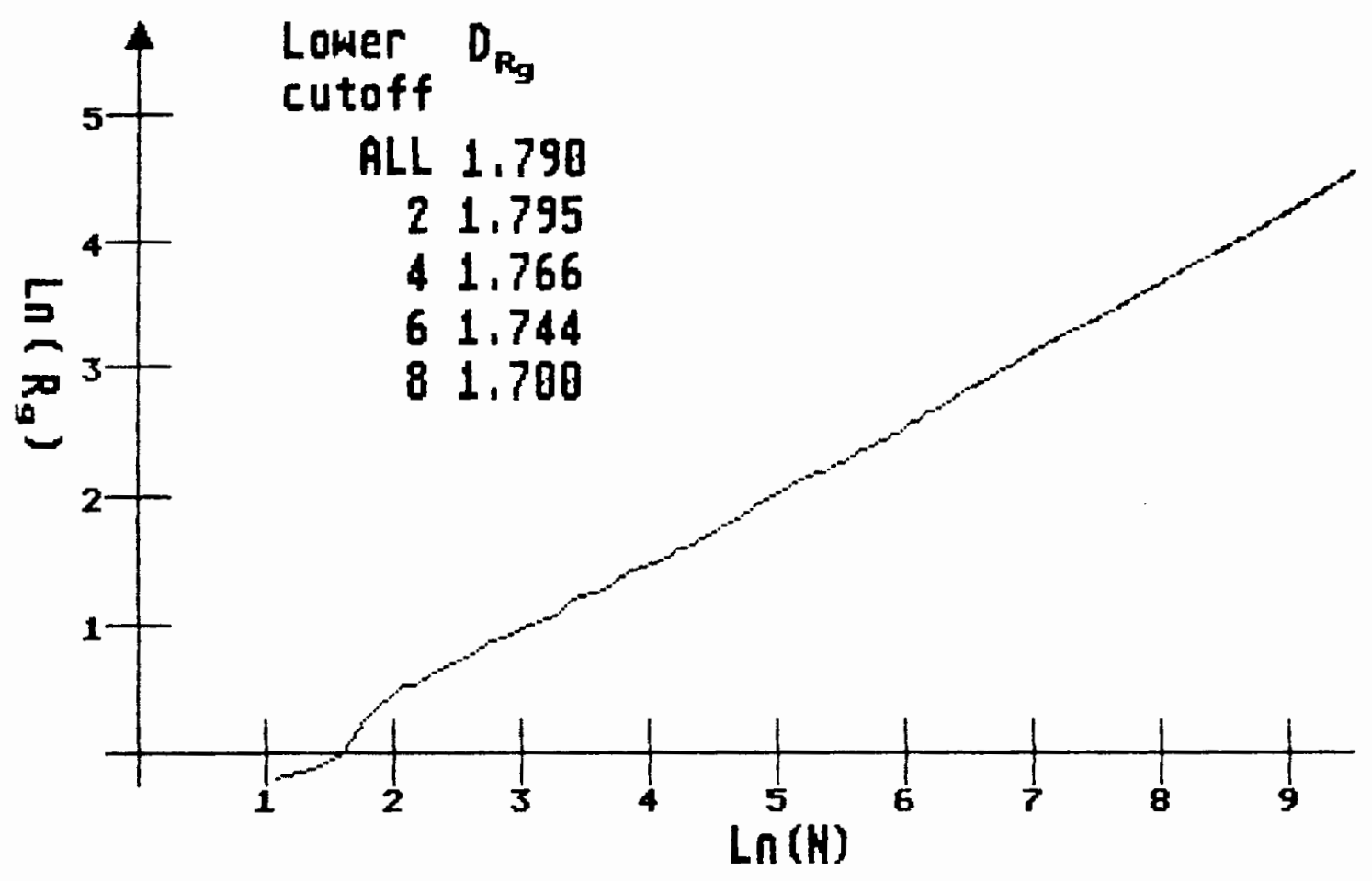

Fiqure 4.3. $\operatorname{Ln}\left(R_{0}\right)$ vs. Ln(N) for aggregate number 23.

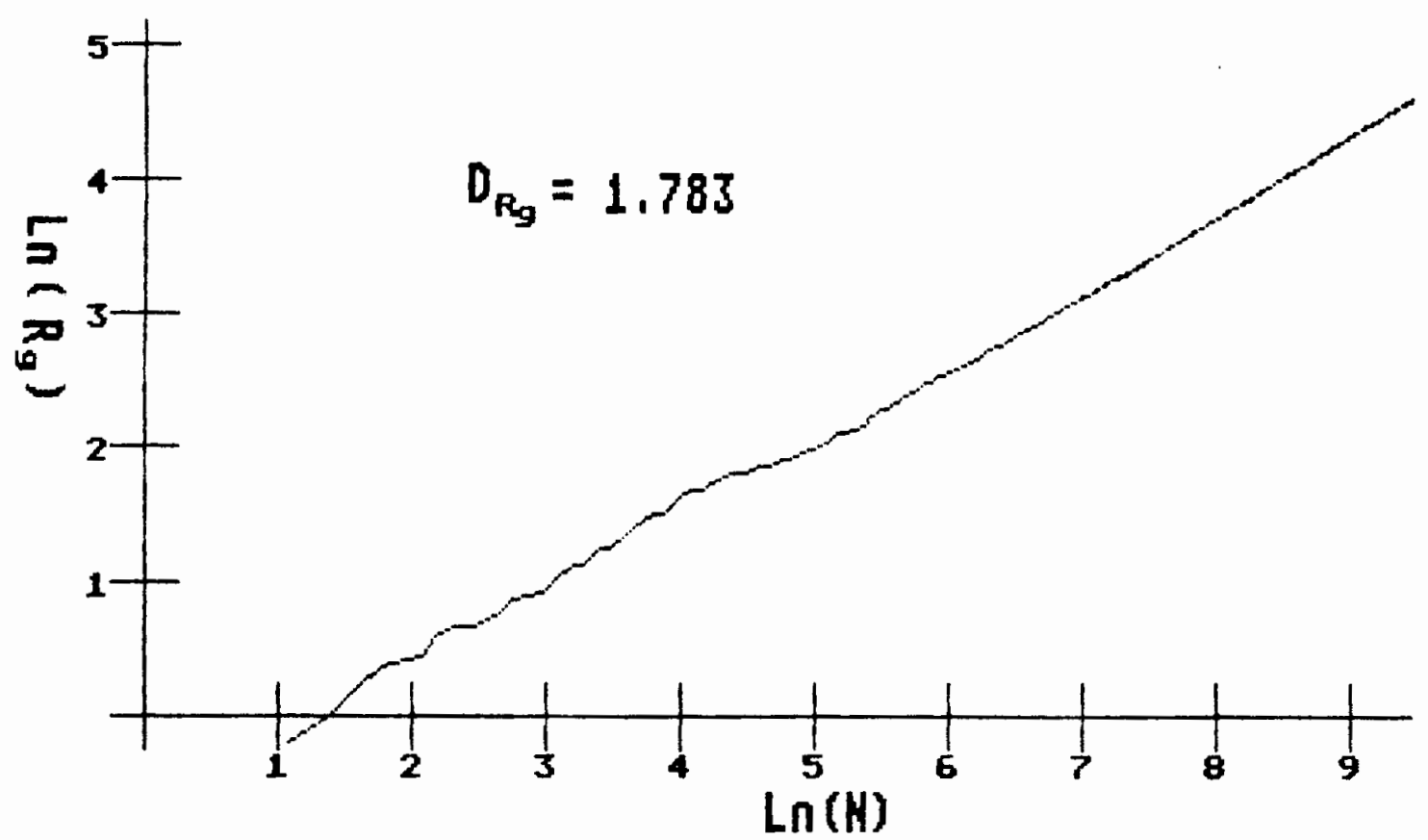

Fiqure 44. $\operatorname{Ln}\left(R_{0}\right)$ vs. $\operatorname{Ln}(N)$ for aggregate number 24. 


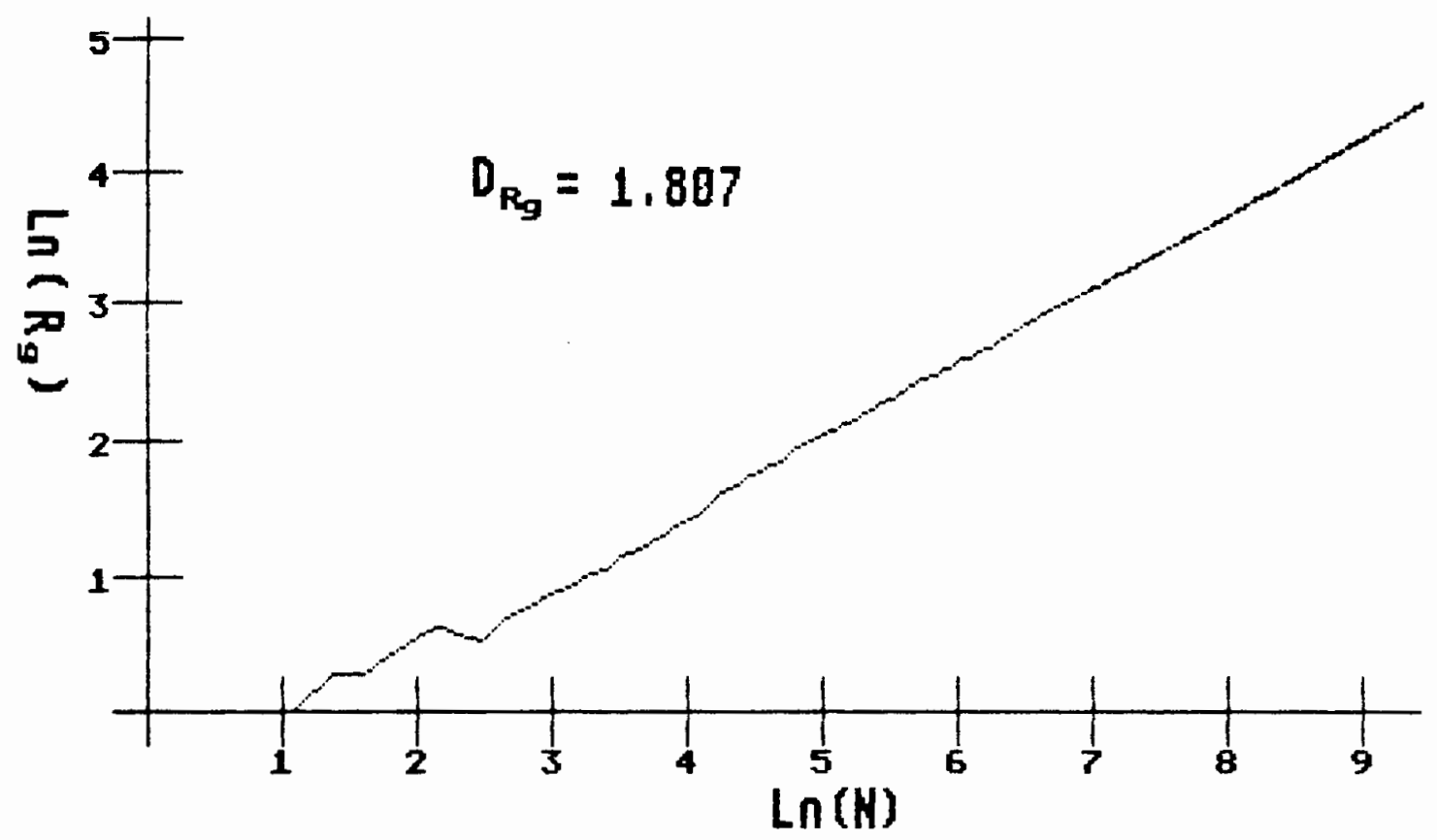

Figure 45. Ln(Ro) vs. Ln(N) for aggregate number 25.

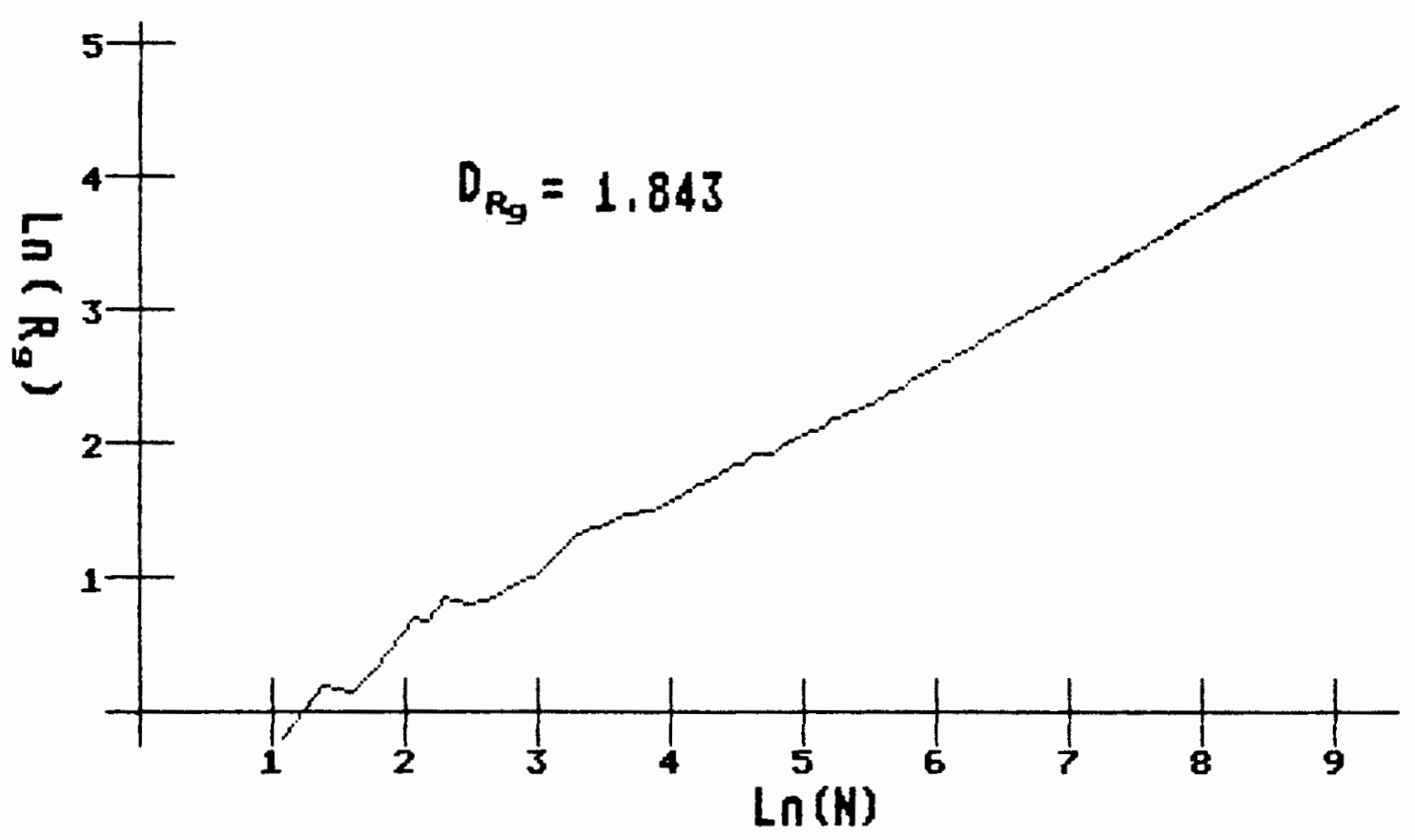

Fiqure 46. Ln $\left(R_{0}\right)$ vs. Ln (N) for aggregate number 26. 


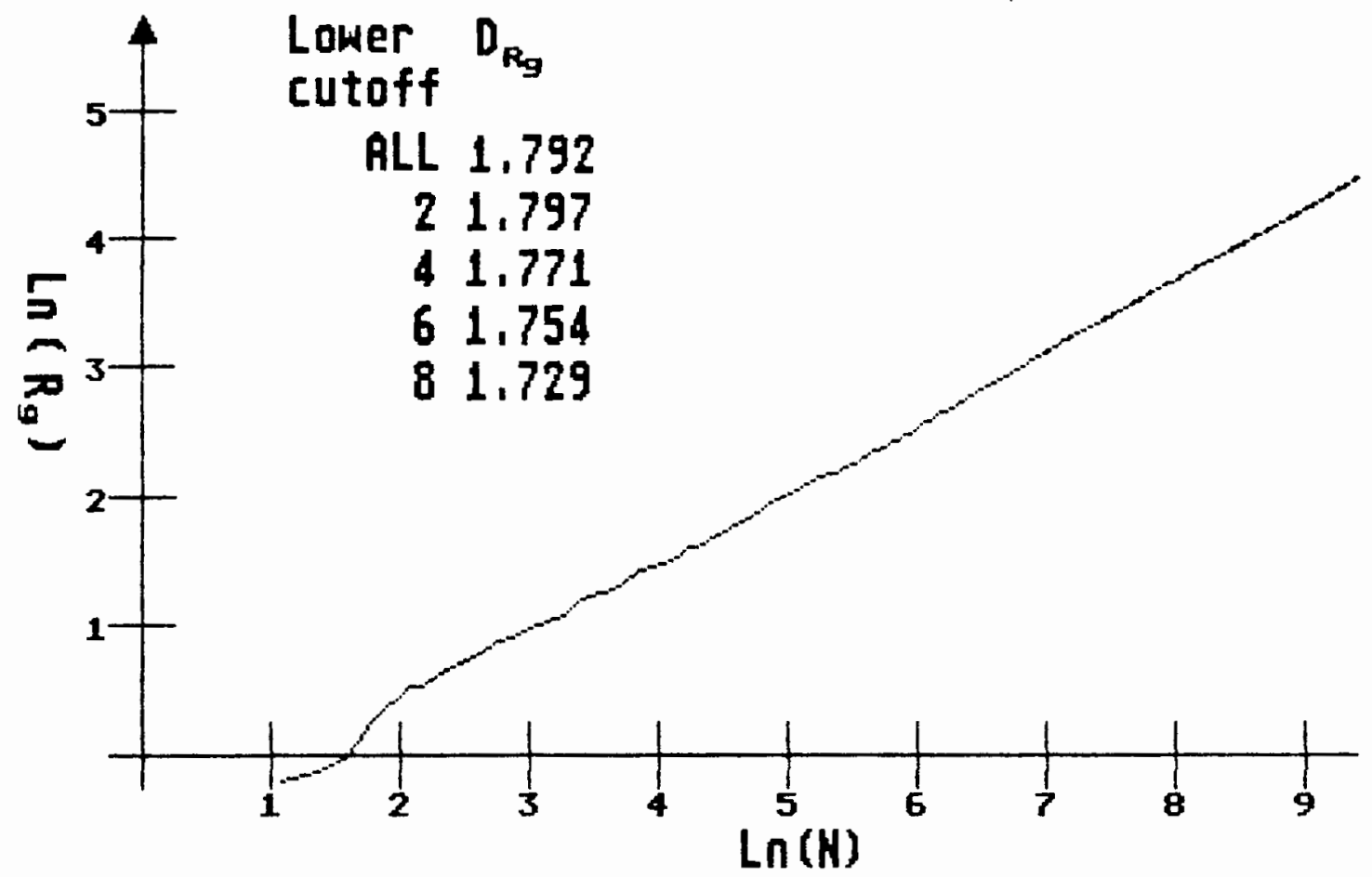

Fiqure 47. $\operatorname{Ln}\left(R_{0}\right)$ vs. $\operatorname{Ln}(N)$ for aggregate number 27.

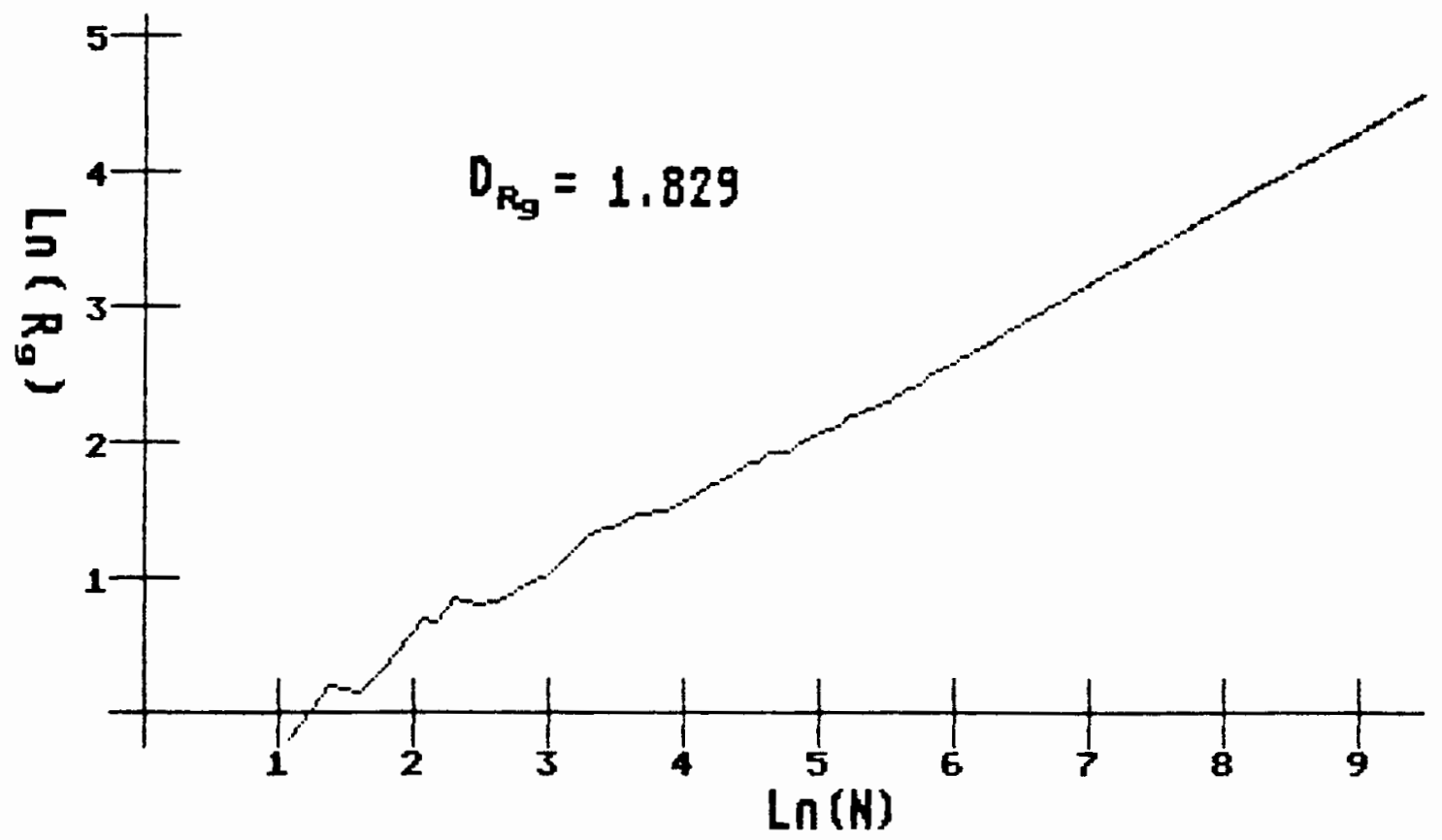

Fiqure 48. $\operatorname{Ln}\left(R_{0}\right)$ vs. $\operatorname{Ln}(N)$ for aggregate number 28. 


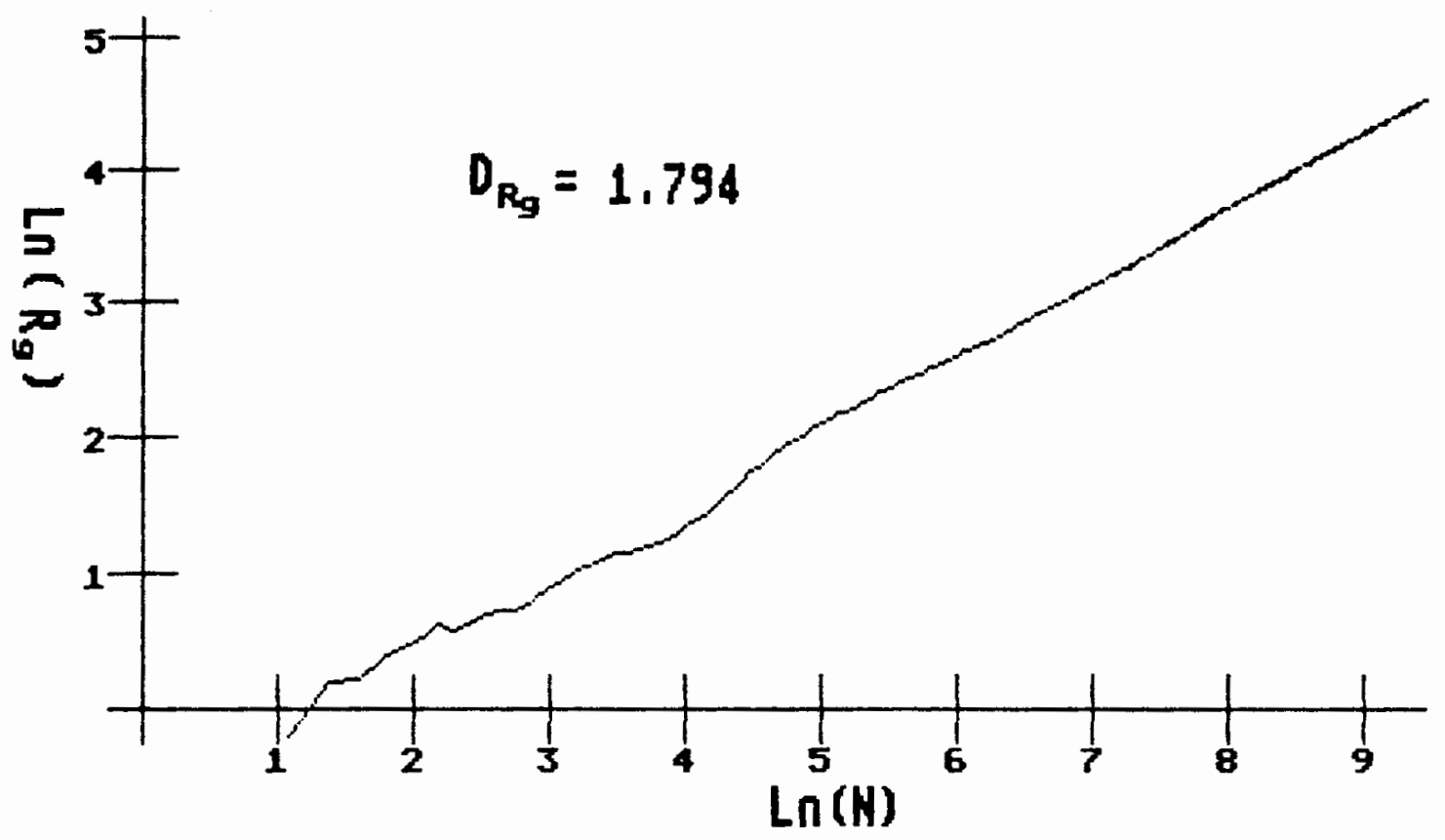

Fiqure 49. $L n\left(R_{0}\right)$ vs. $L n(N)$ for aggregate number 29.

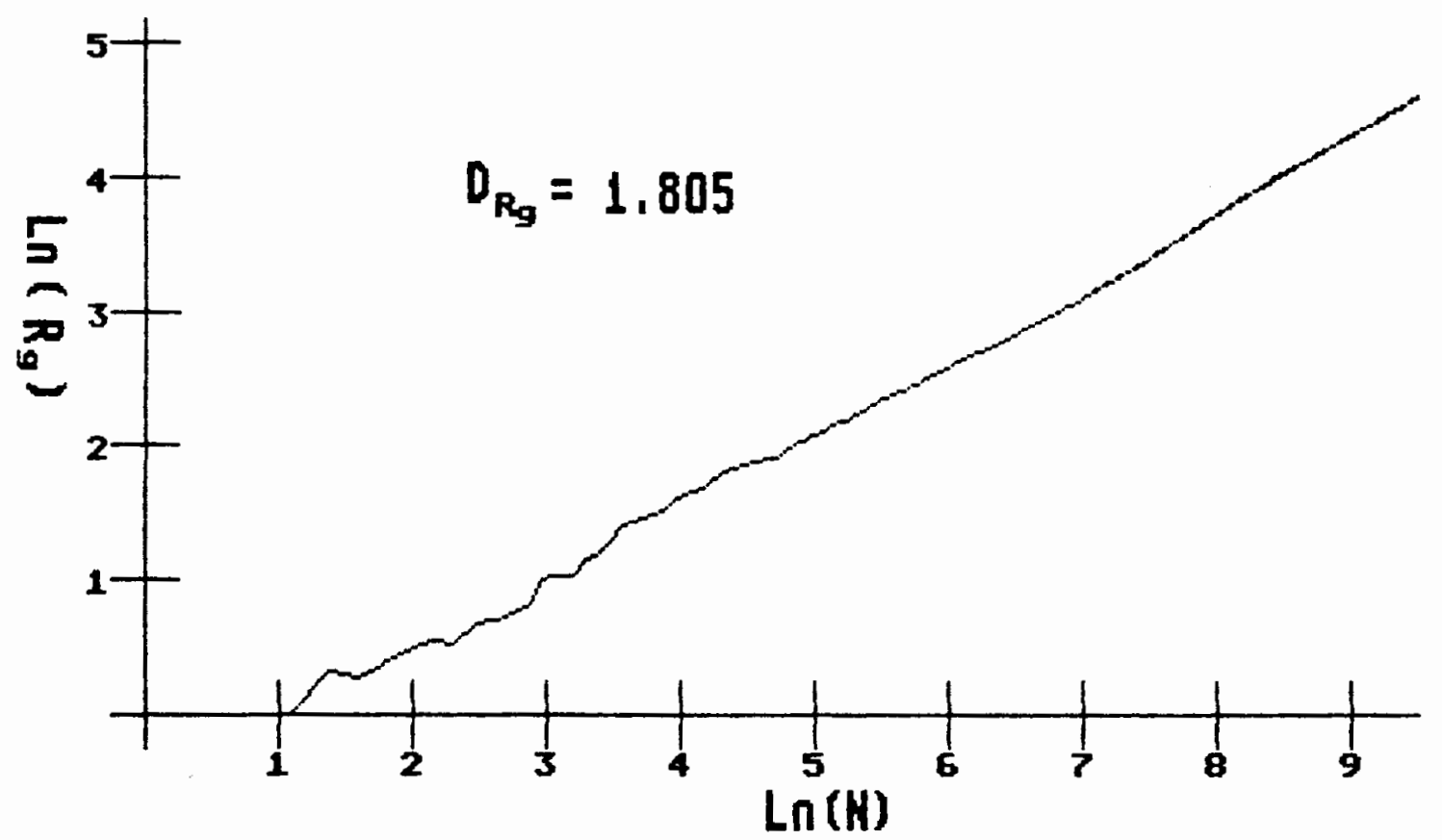

Fiqure 50. Ln(Ro) vs. $\operatorname{Ln}(N)$ for aggregate number 30. 
APPENDIX $\mathbf{C}$

GRAPHICAL DATA

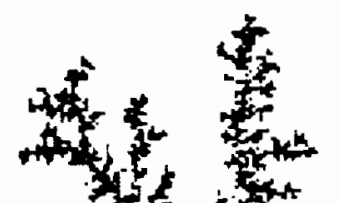

7.

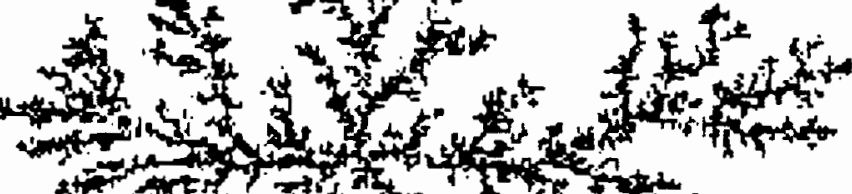

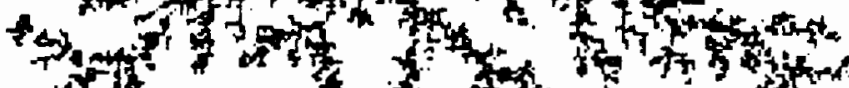
$\frac{1+4}{4}$
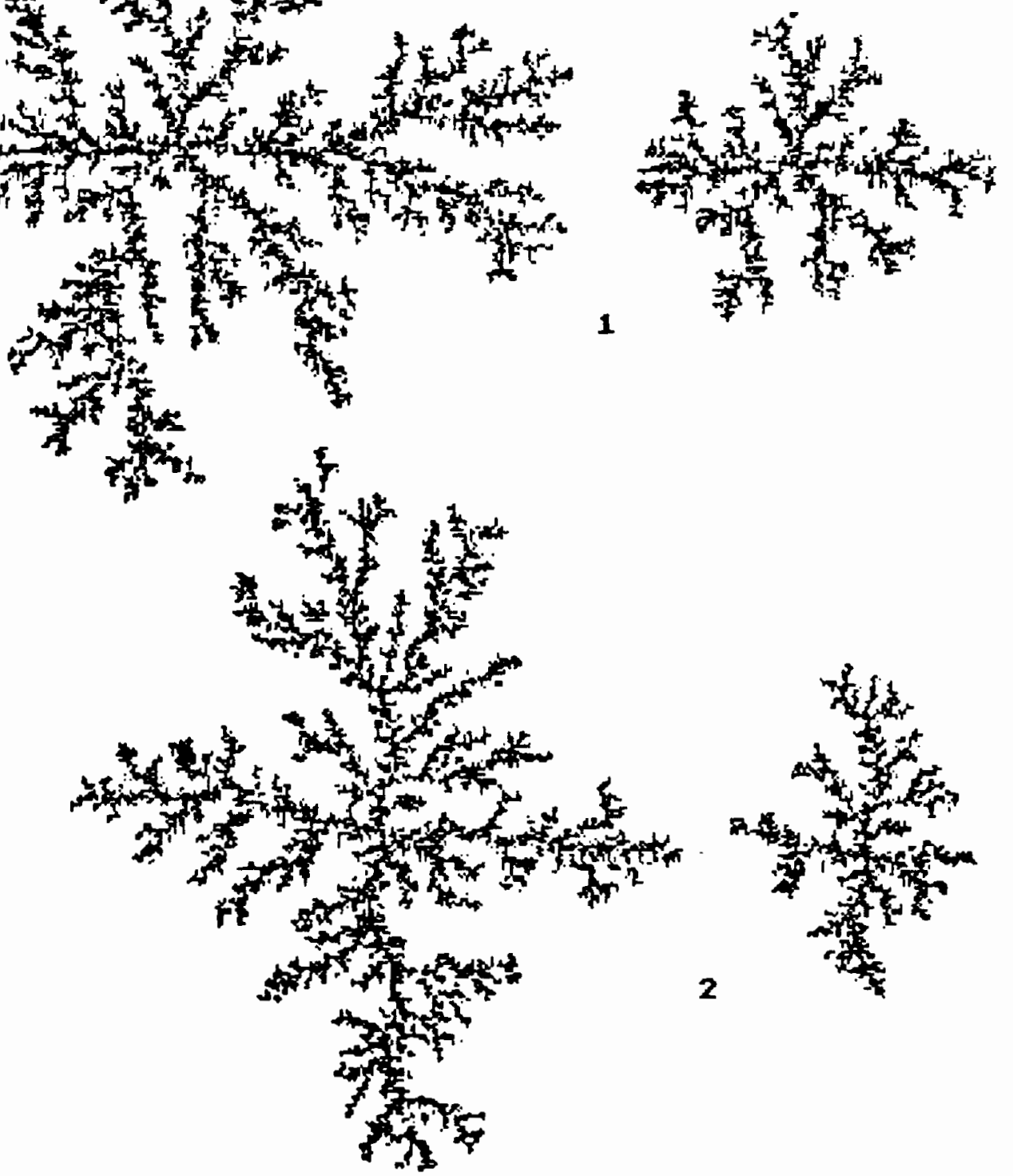

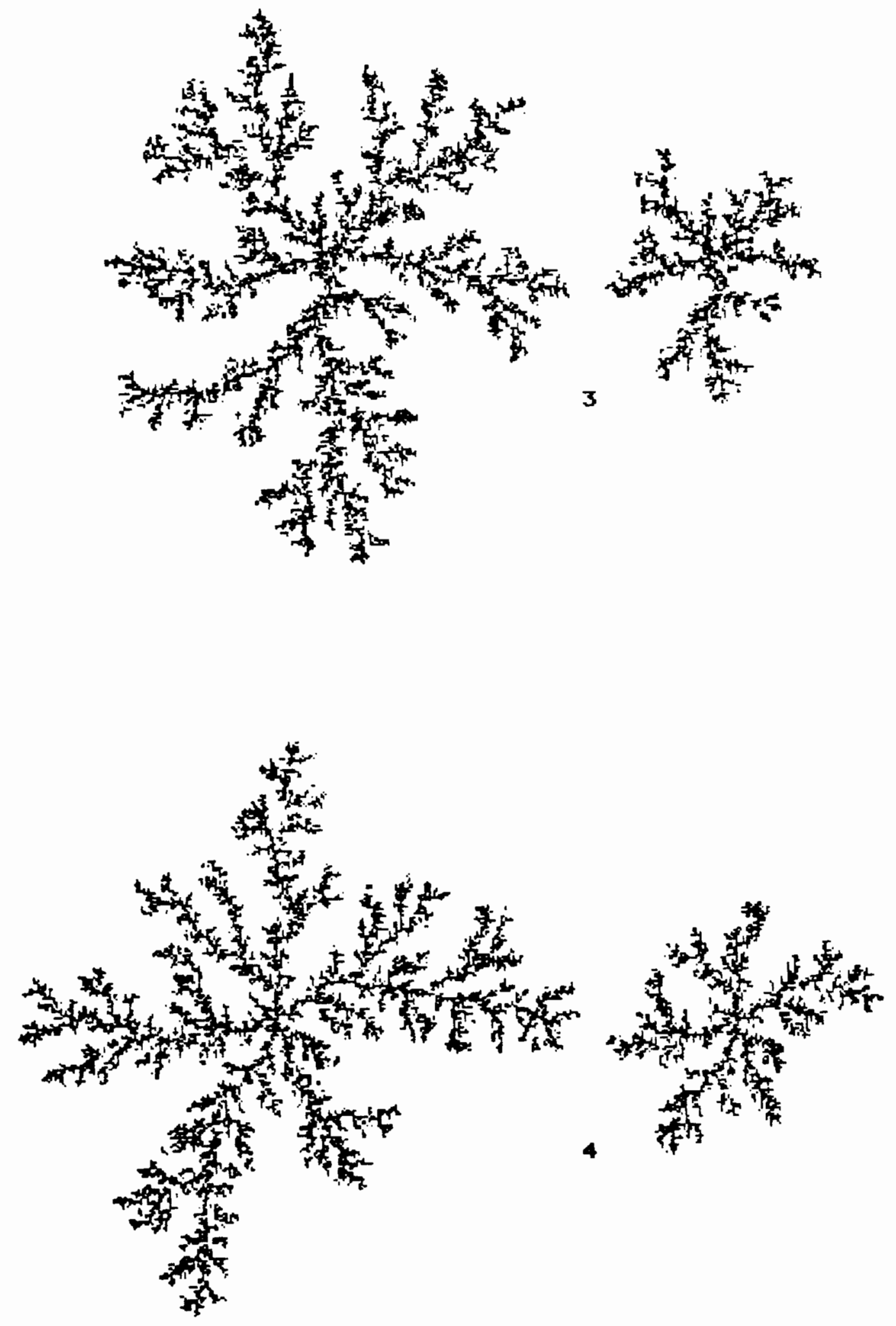

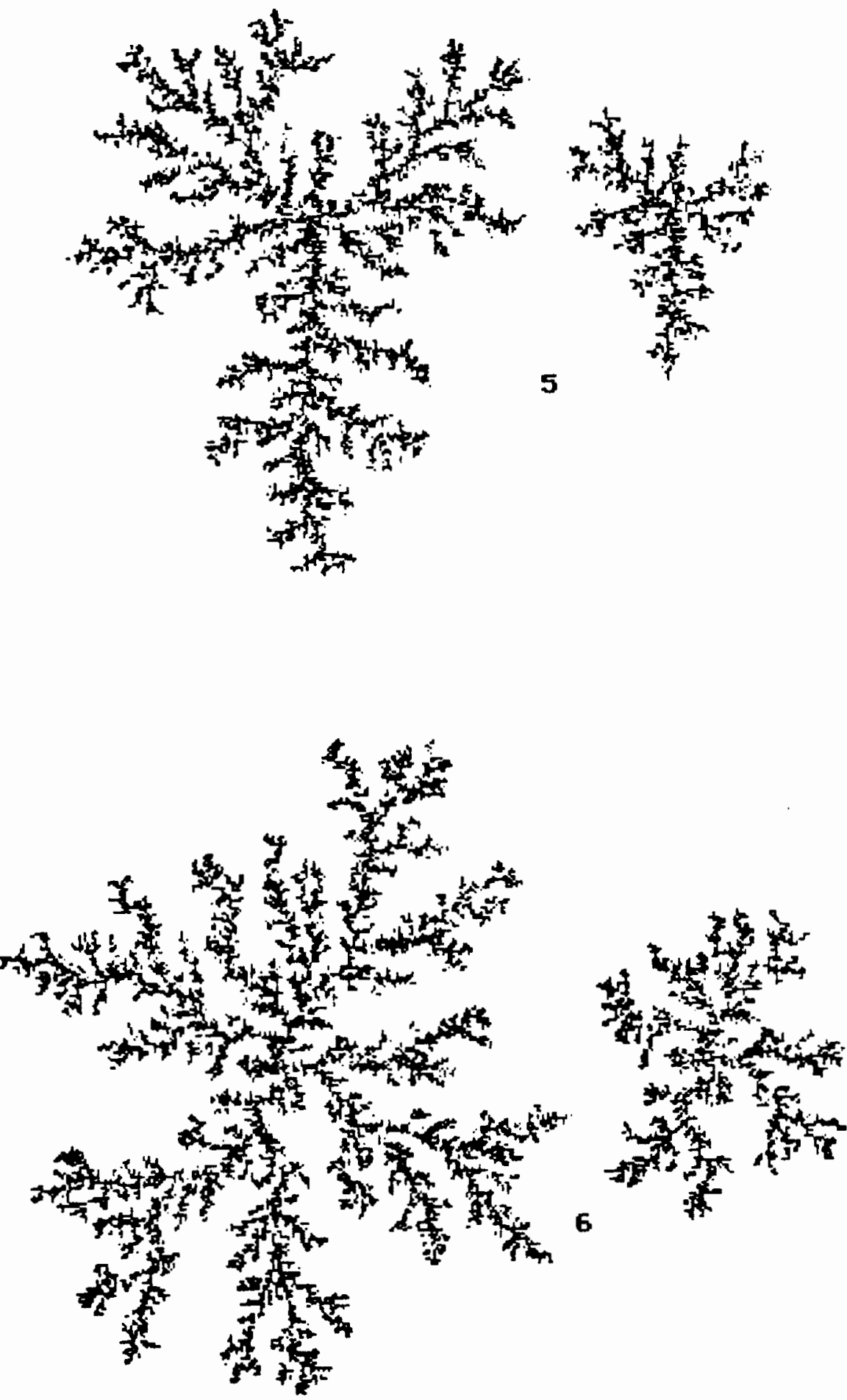

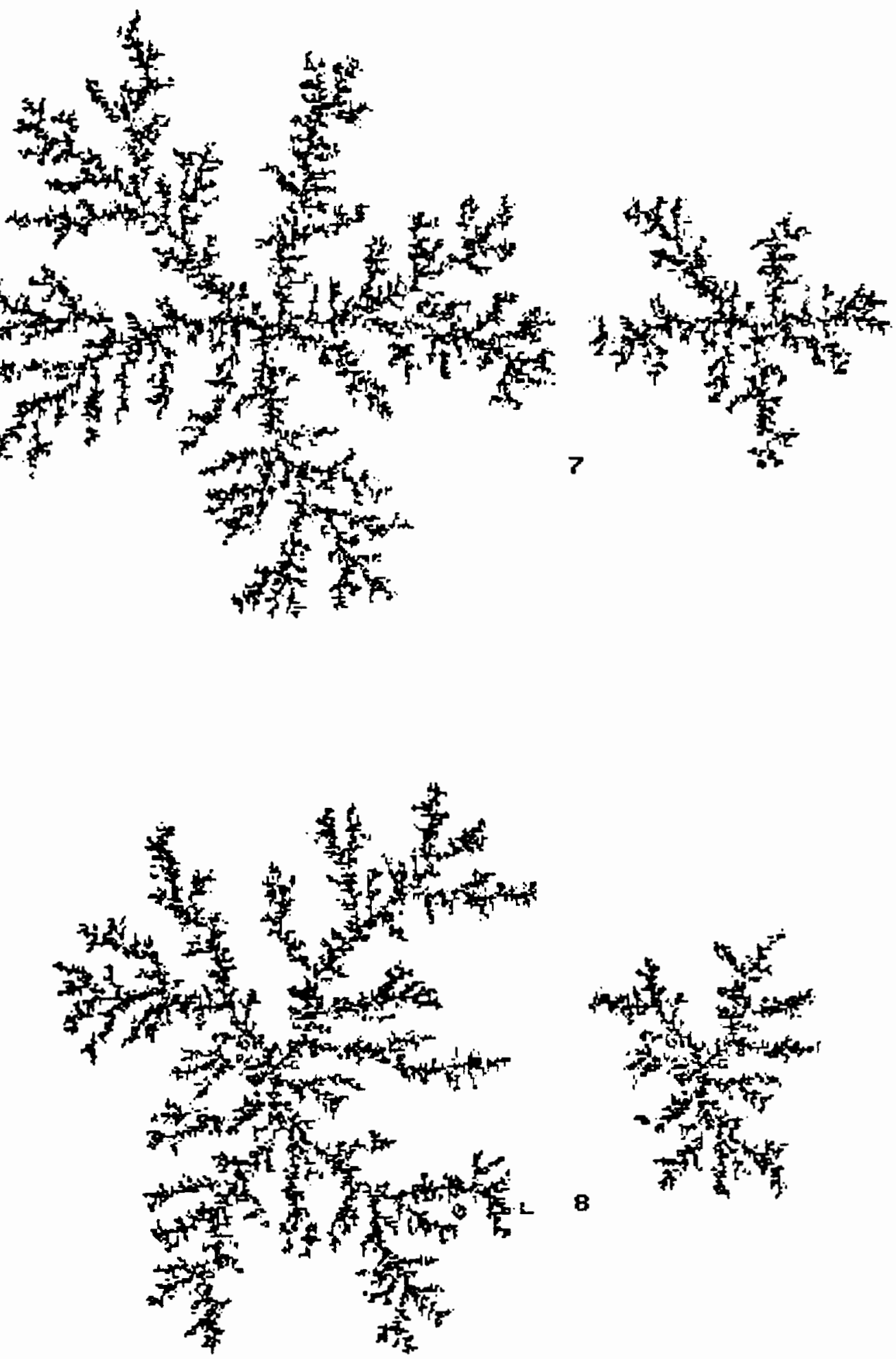

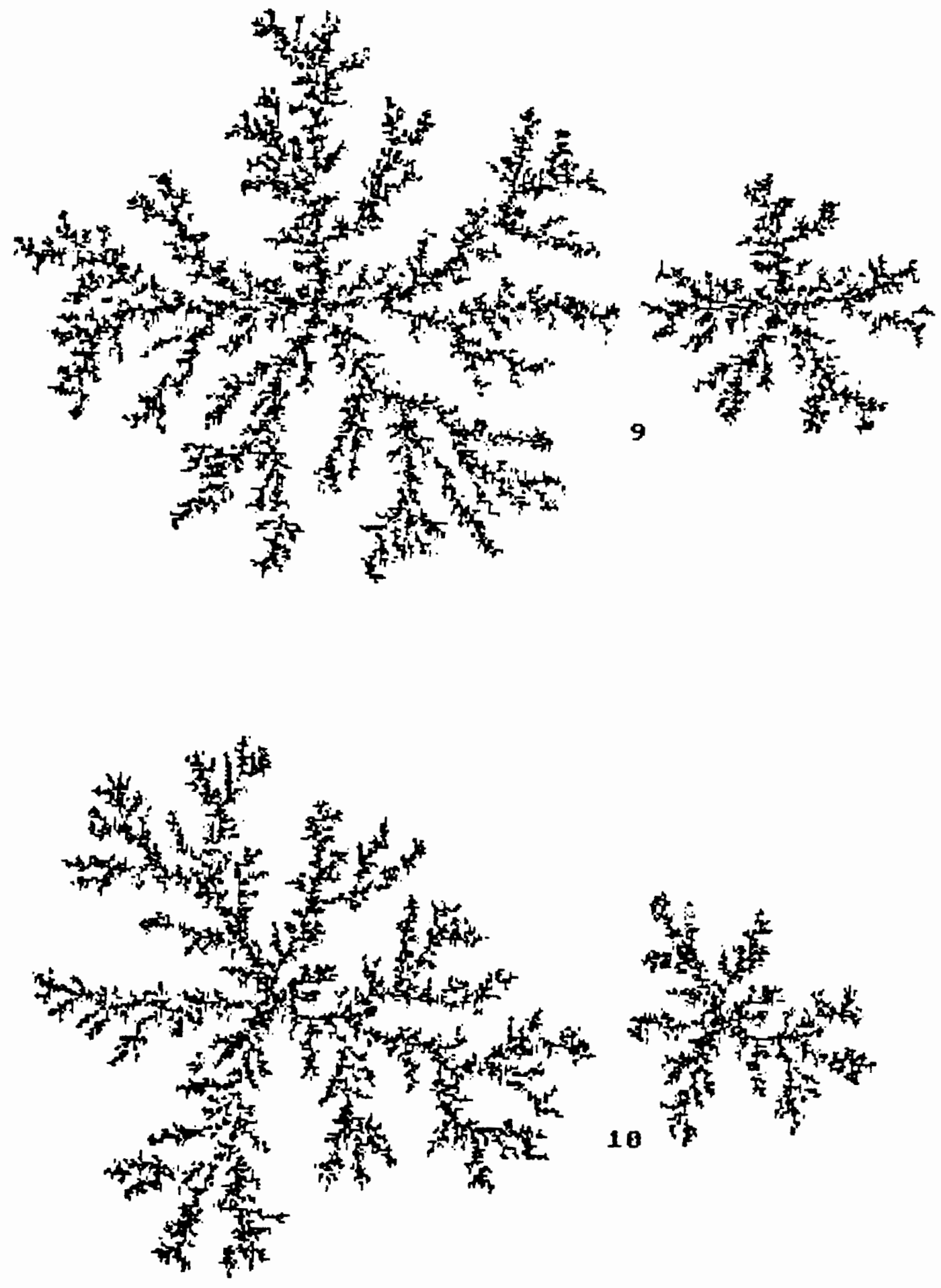


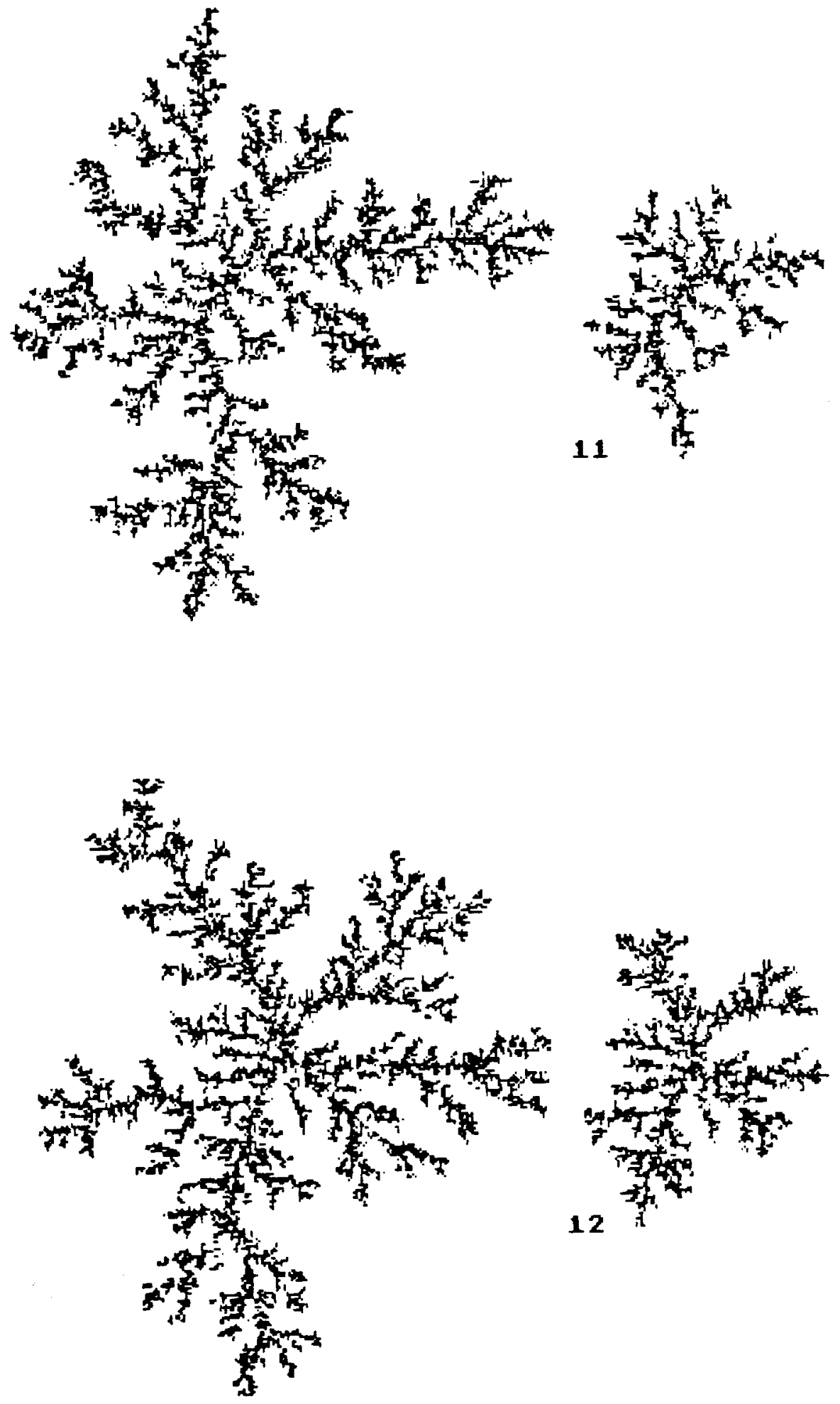



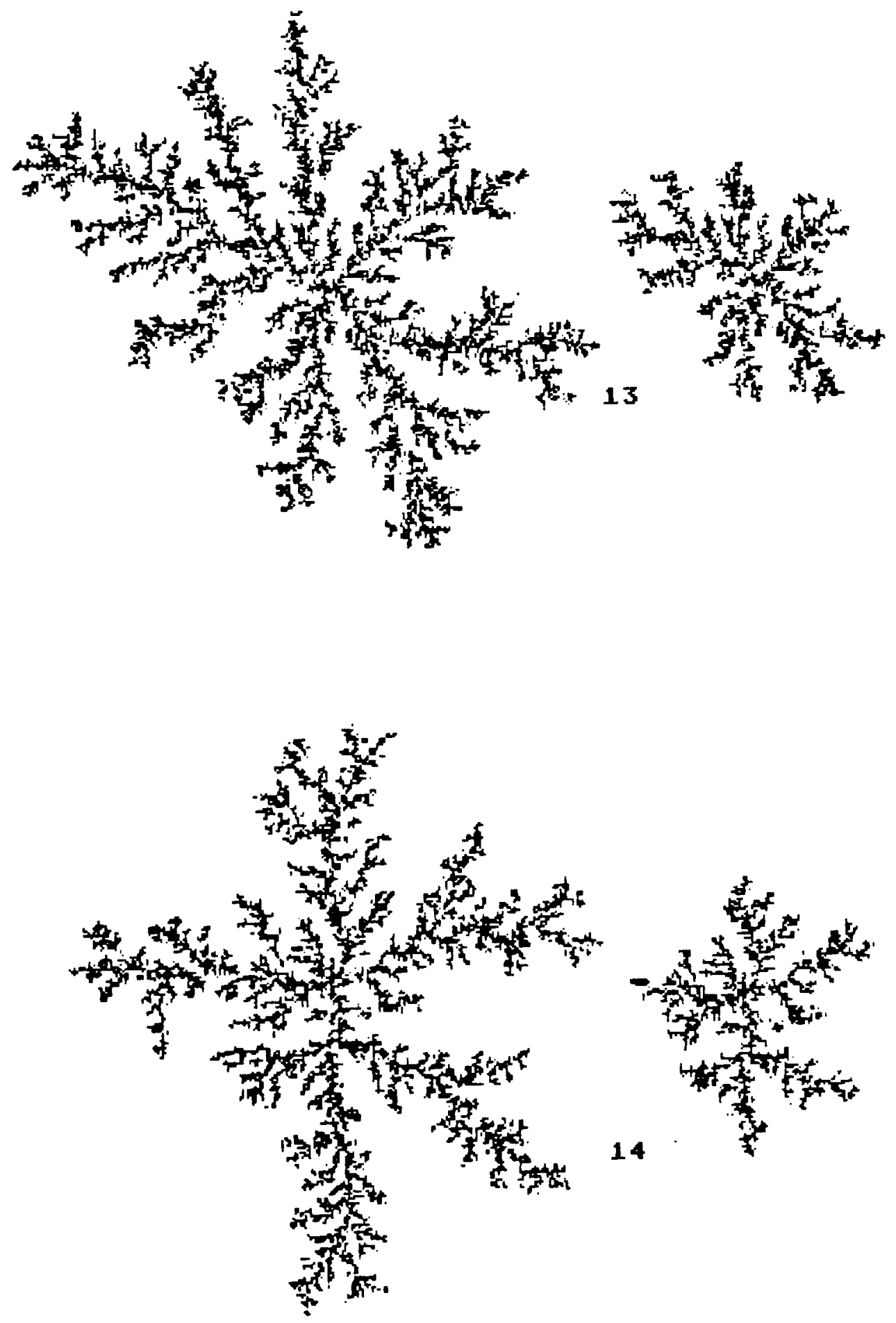

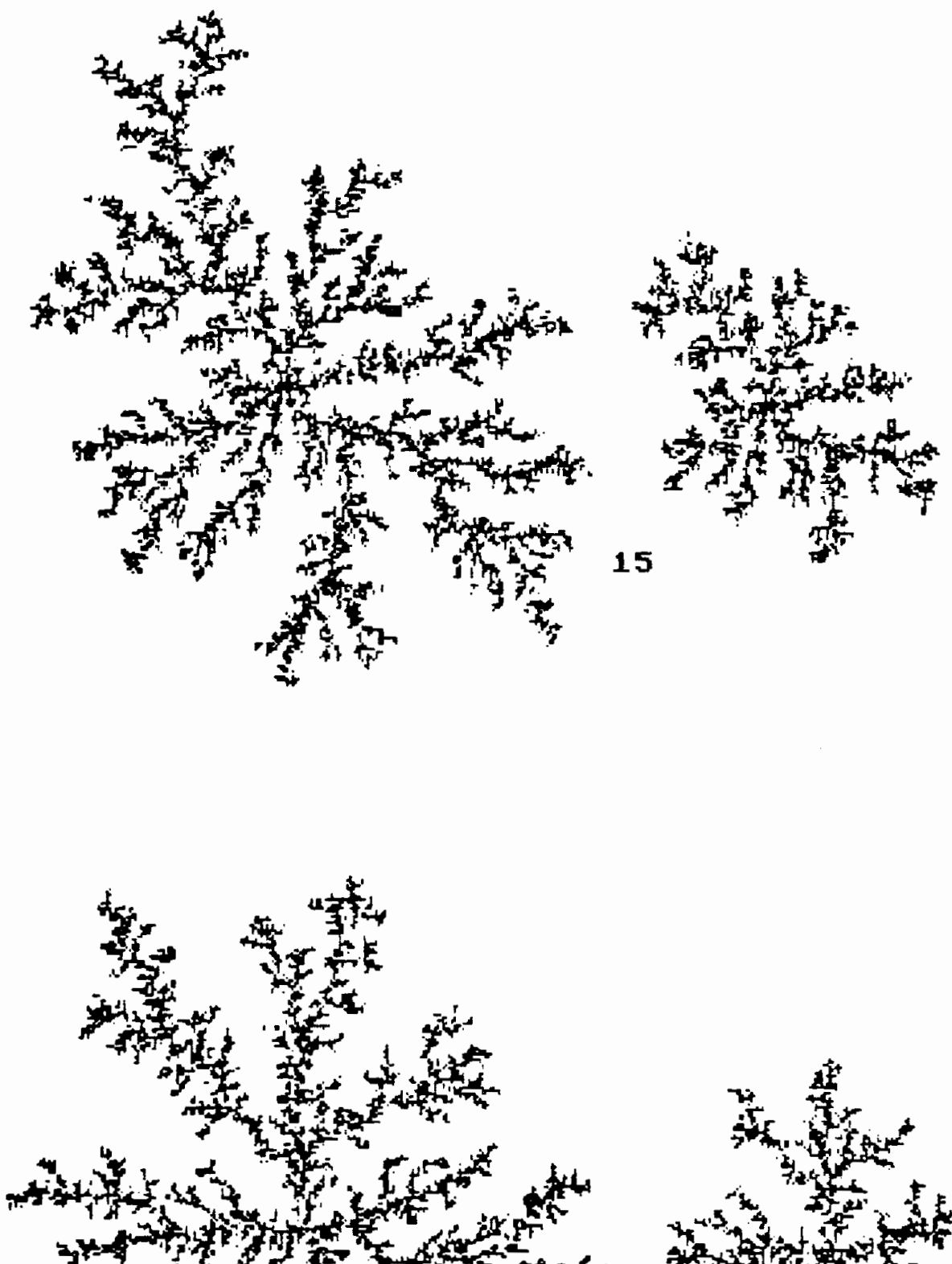
24ty

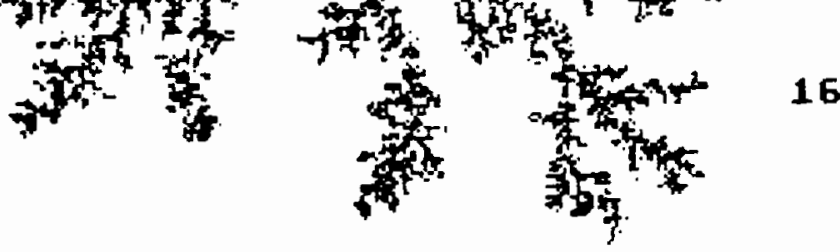

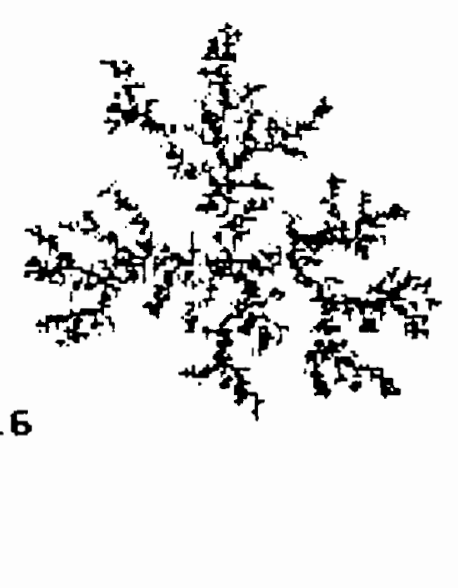



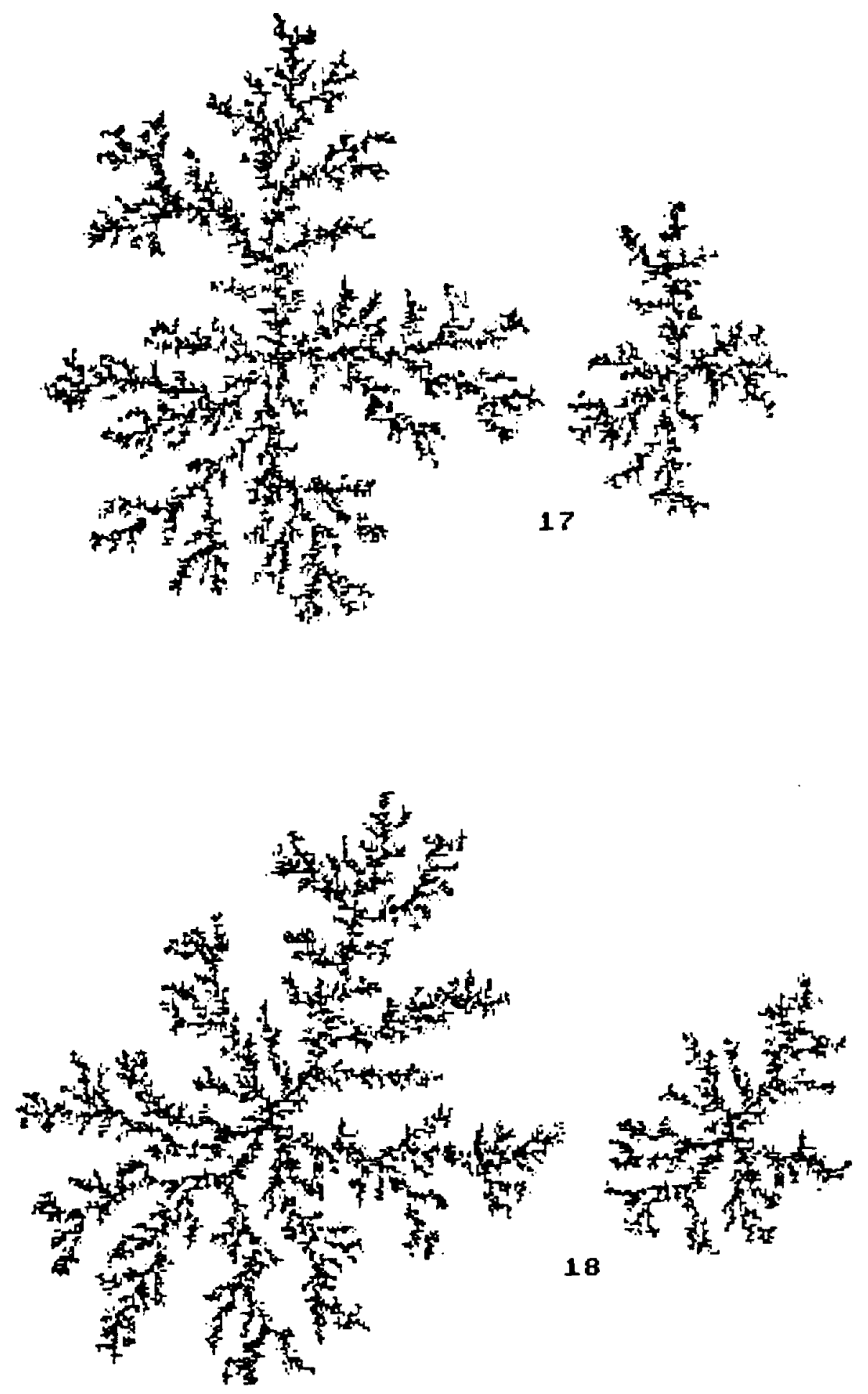


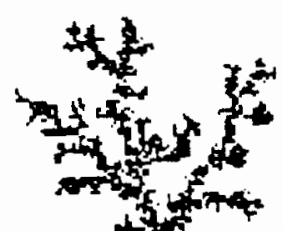

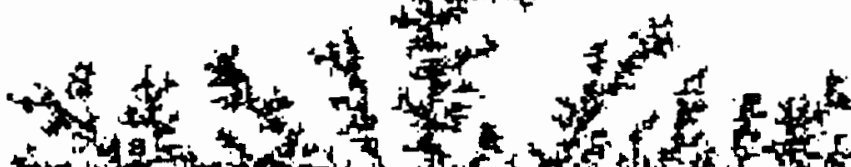

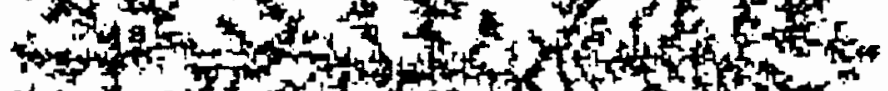
iftitis

$$
\text { (1) }
$$
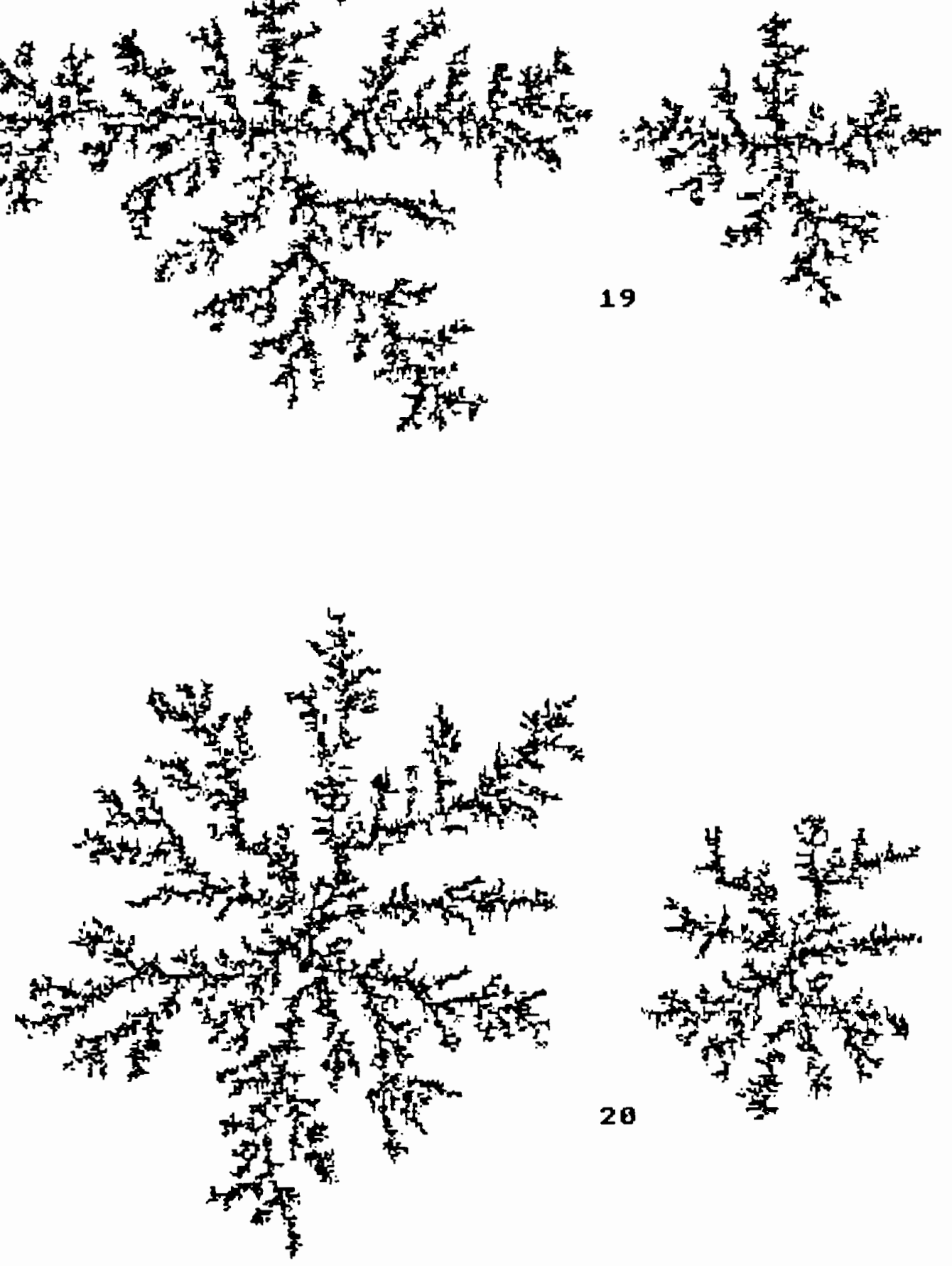

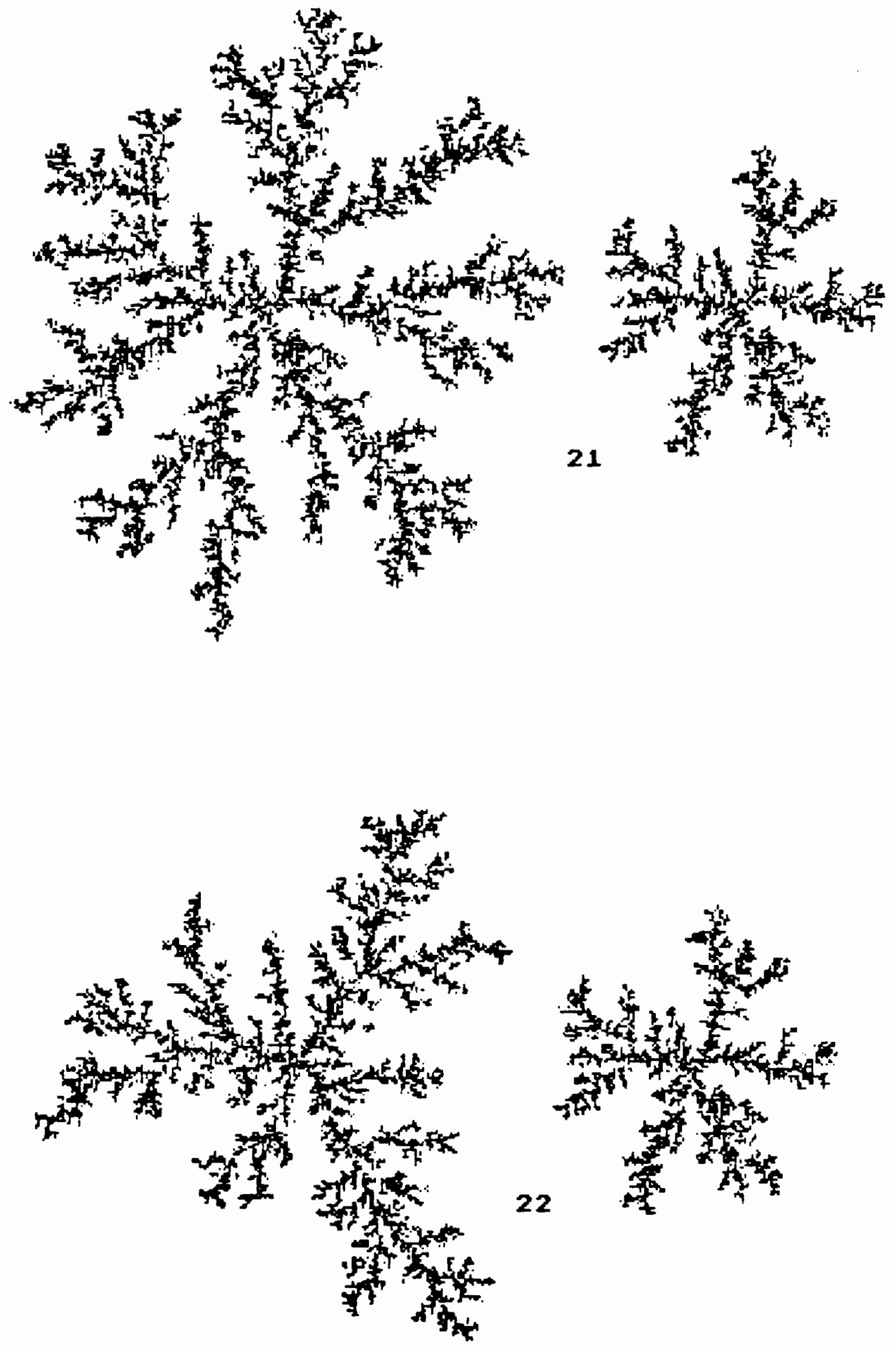

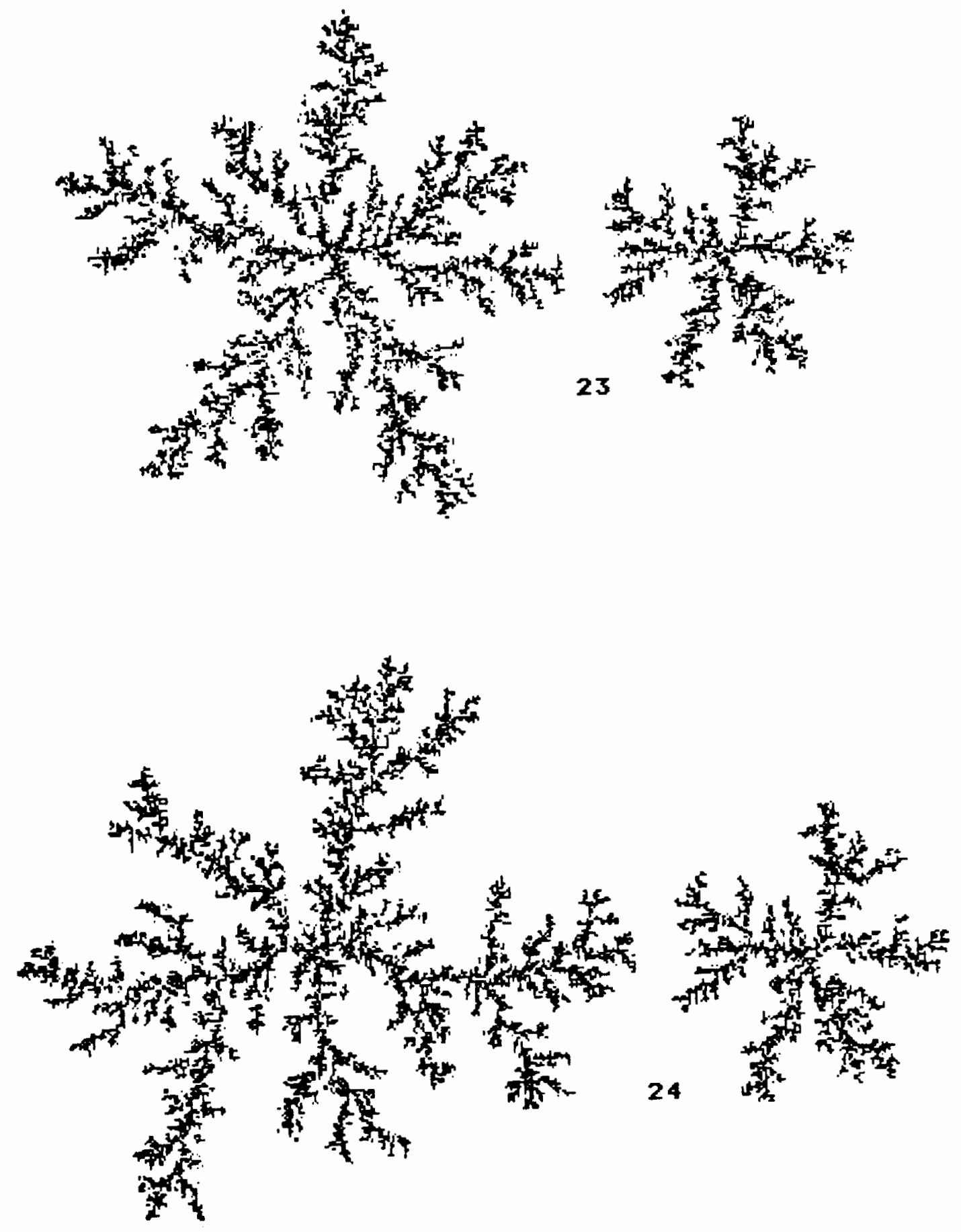

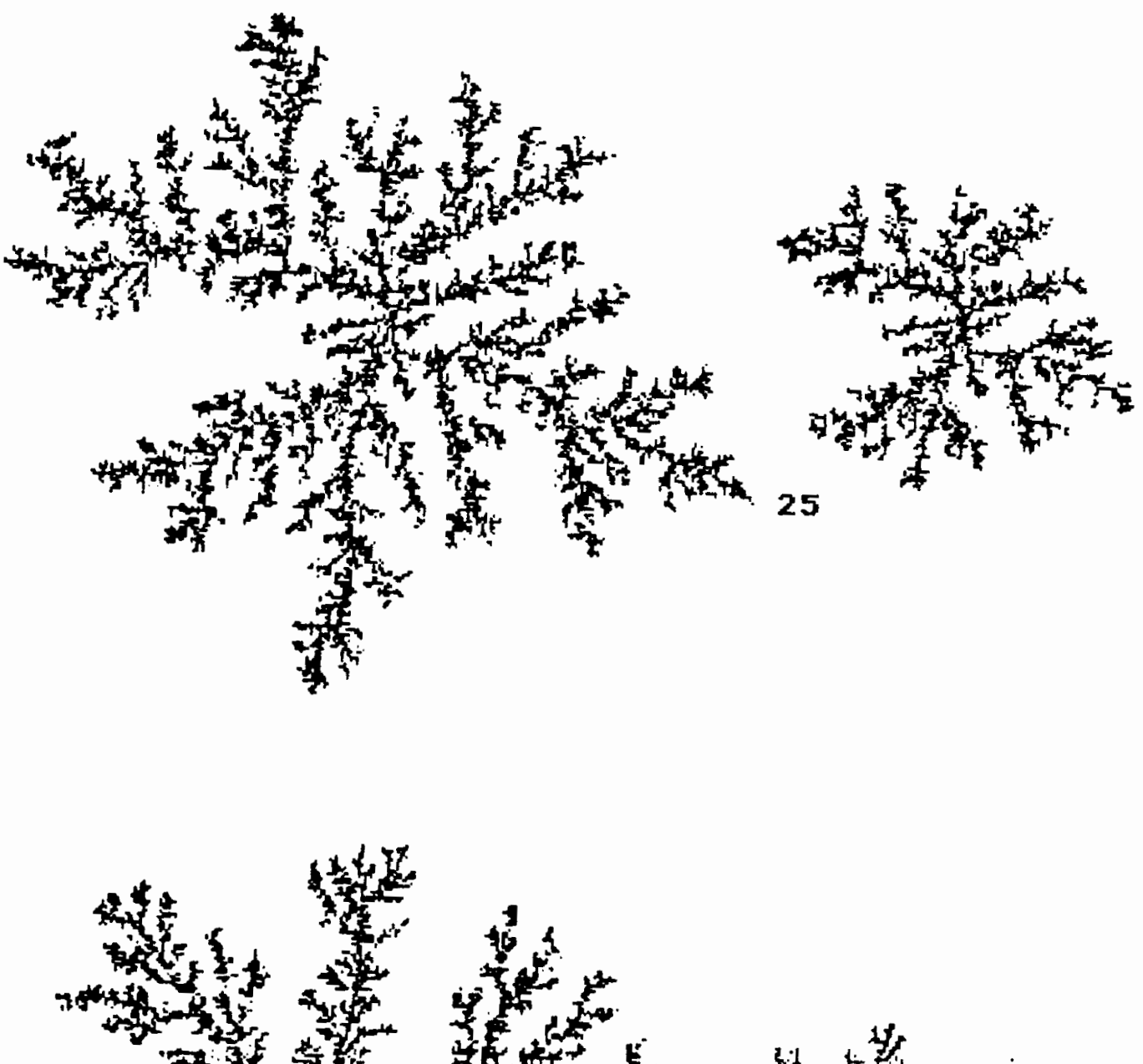

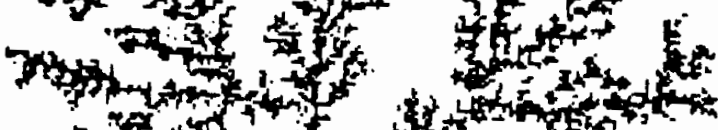
rete

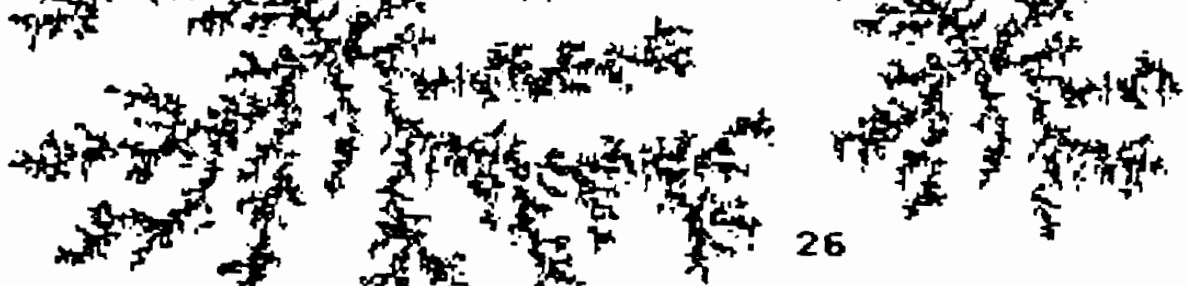
ats at is

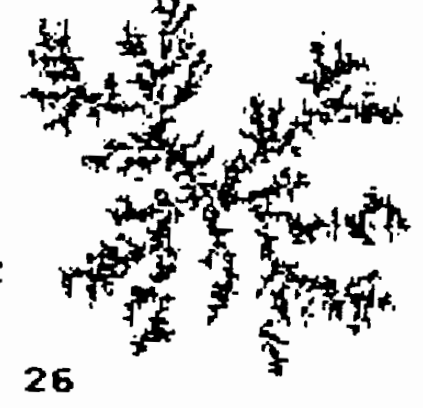
瀑禁 

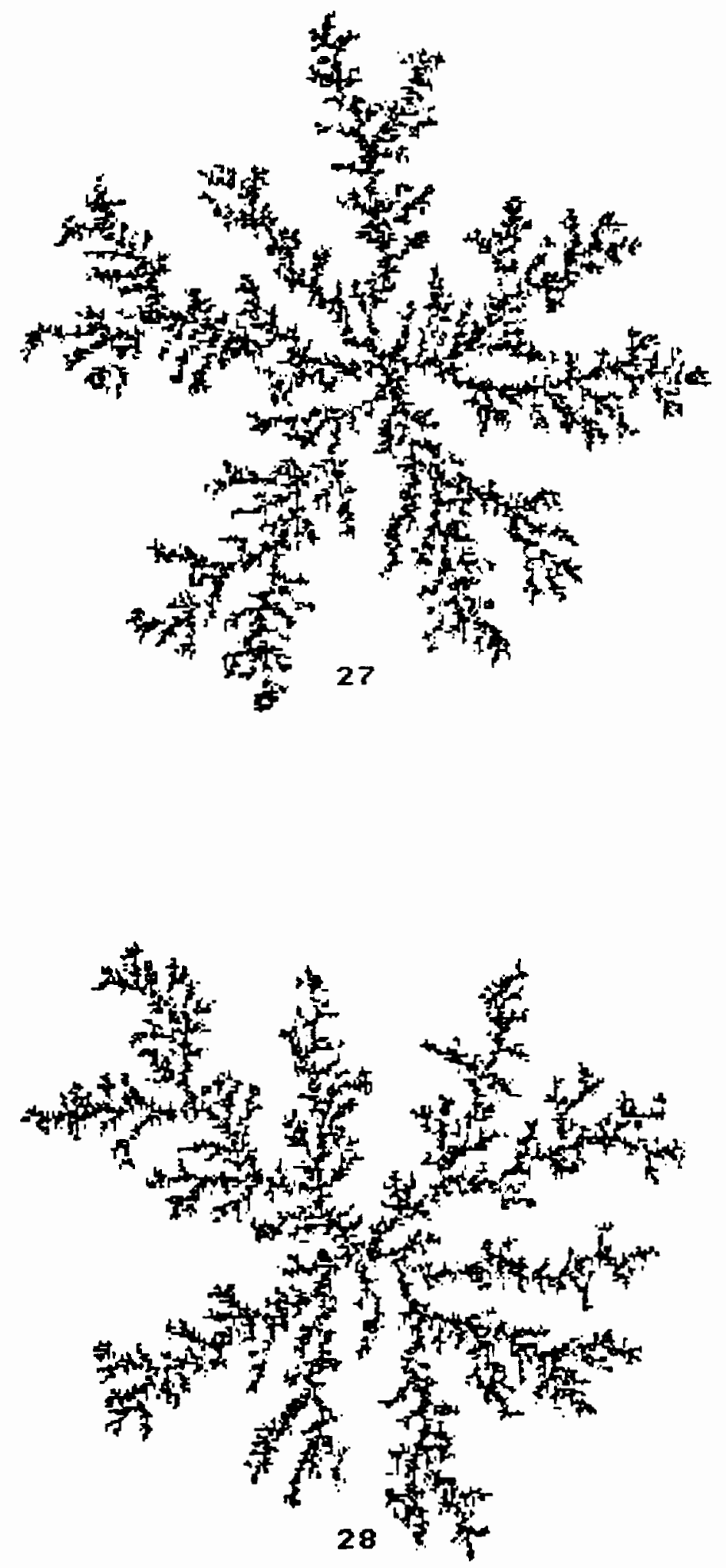

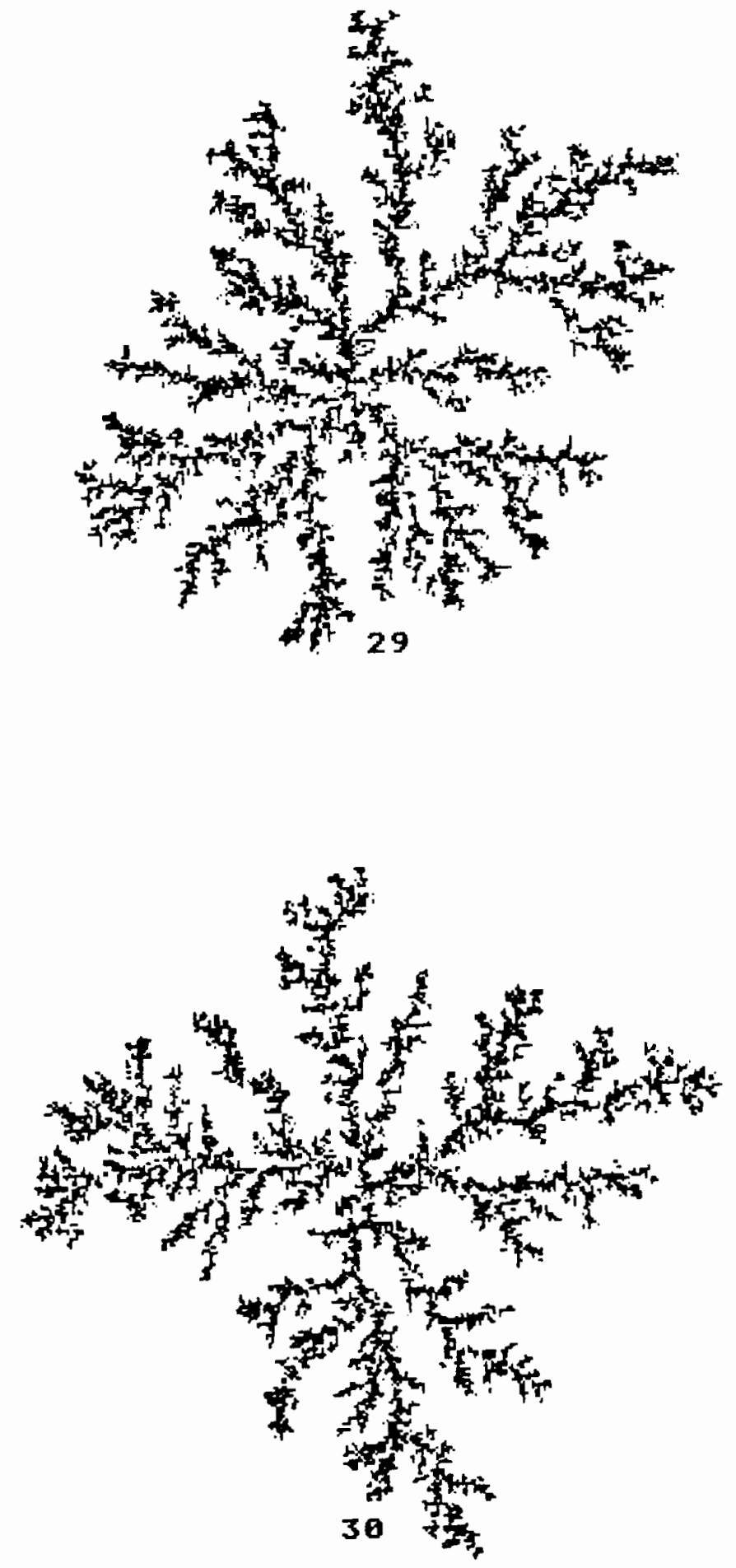


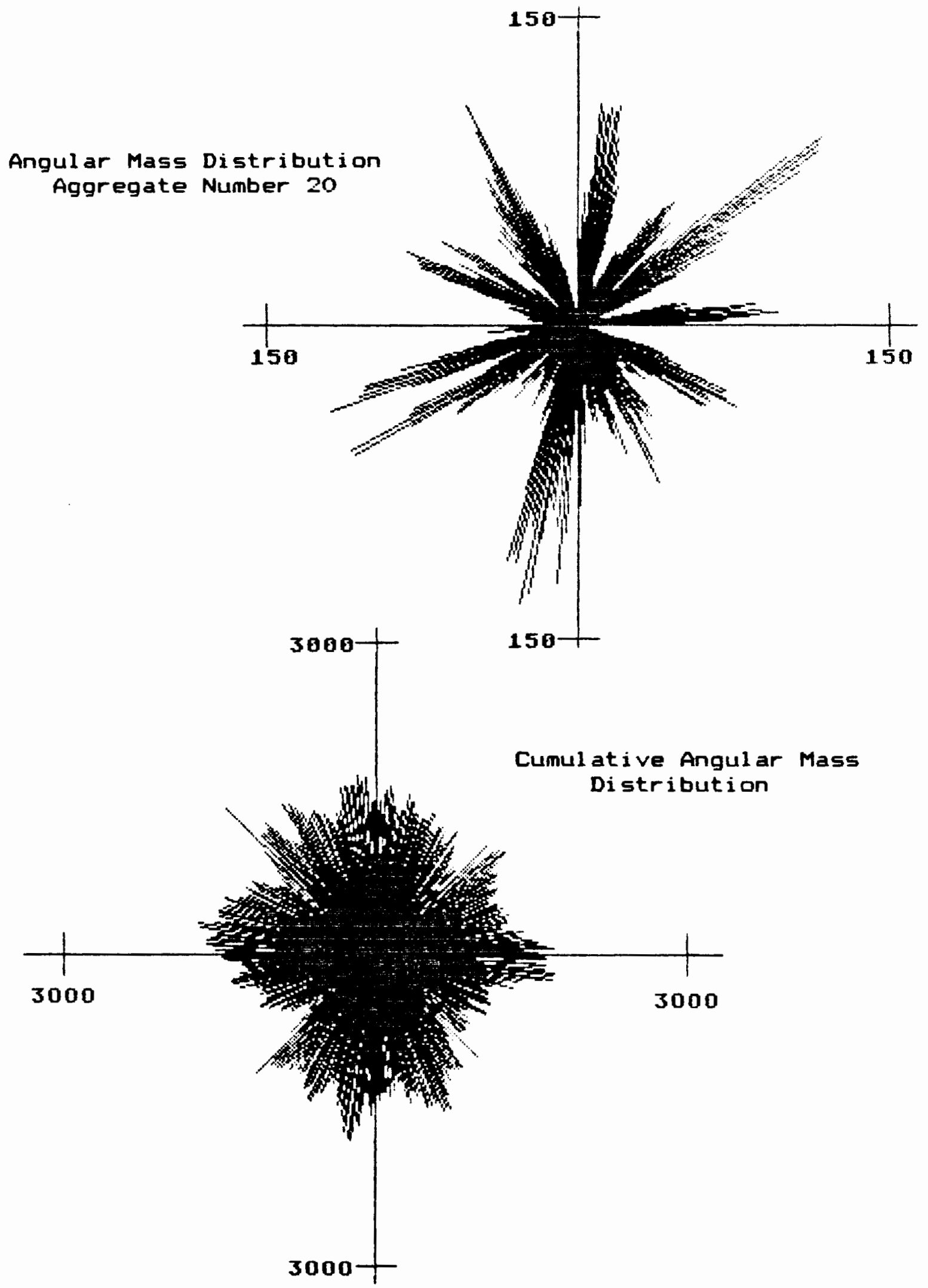


APPENDIX D

ADDITIONAL RADIUS OF GYRATION ANALYSIS

The radius of gyration is defined as the average sum of squares of the distances from the center of deposition to each deposit. The exact calculation of the radius of gyration dependence on the number of deposits would have necessitated $\mathbf{N}$ recalculations for the center of deposition and consequently a much longer process time. The assumption was made that the average center of deposition, for a large sample of aggregates, would be near the lattice origin. However, as discussed above, the average center of deposition was appreciably displaced from the origin. Moreover, the discrepancy in the fractal dimension, as based on this approximate radius of gyration, was unacceptable. In order to obtain a reasonable bound on this error it would be necessary to be able to estimate the dependence that this displacement had on the number of deposits. Analysis of the composite of all the aggregates and also of aggregate number 20, indicated that this displacement was not even monotonic. Instead of analyzing this distribution further, and estimating the fractal dimension using data that was known to be in error, it became obvious that it would be most prudent to recalculate the exact radius of gyration for a 
selected number of deposits and to obtain an approximate fractal dimension based on exact data. The following provides the details of the above argument and the resulting analysis.

The parallel axis theorem for the moment of inertia, $I=I_{c . m_{-}}+N^{\circ} L=$, where $L$ is the displacement from the center of mass, c.m., can be utilized to modify the radius of gyration, $R_{\theta}=(I / N)^{2 / 2}$. The dependence, $L=L(N)$, was not obtainable, only $L\left(N_{\max }\right)$ was known. Although, regression over all N of the deposits would have been the preferred method, however, without the corrections based on $L(N)$, the results would have been systematically in error. A twopoint approximation for the slope of $\operatorname{Ln}\left(R_{\sigma}\right)$ vs. Ln(N) could have been obtained lutilizing the parallel axis theorem with the final displacements of the centers of deposition) by using the final deposits of the small and large forms of the same aggregate (Slope $=$ Slope $\left(N_{\max .}\right)$ ). However: recalculation of the radius of gyration based on the center of mass for a limited number of points would not have required an excessive amount of time. Thus, the radius of gyration program was modified and these data points were calculated directly. A more thorough analysis of aggregate number 20 was also performed in order to provide an additional comparison. These slopes, of 26 independent aggregates, were averaged. The result was compared with the slope of the least squares regression line based on the plot 
of the $\mathbf{5 2}$ data points. Any discrepancy here would indicate correlations between those data points associated with the large and small forms of the same aggregate.

The result of the two-point slope calculation for aggregate number 20 is, slope $=0.592$, which gives a fractal dimension of $D_{n o}=1.69$. The results based on the approximate radius of gyration for aggregate number 20 from Appendix B, are, for the small aggregate, $D_{n o}=1.83$, and for the large aggregate, $D_{\text {no }}=1.81$, their average is 1.82 . Even though there is considerable variation among any of the individual deposits, this discrepancy is substantial. Aggregate number 20 was sampled at 20 increments of $5 \%$ of $N_{\max }$ and this data was analyzed using least squares. The resulting fractal dimension based on the slope of the regression line is $D_{n e}=1.67$. The coefficient of determination, $R^{2}$, for the regression is 0.95 . This is in close agreement with the more approximate result based on the two-point slope calculation. Thus, the two-point slope method yields credible results. The data obtained for aggregate number 20 is listed below in Table IV and the graph is in Figure 51.

The average of the two-point slope calculations of aggregates numbers 1 to 26 , inclusive, using the final deposits of the small and large forms of each aggregate is, slope $=.58 \pm .02$. This result yields a fractal dimension of $1.73 \pm .06$. The raw data for this calculation is 1 isted 
TABLE IV

CORRECTED RADIUS OF GYRATION RESULTS FOR AGGREGATE NUMBER 20

\begin{tabular}{|c|c|c|c|c|}
\hline$\underline{Z N}$ & DEPOSITS & $\ln (N)$ & $\operatorname{Ln}\left(R_{0}\right)$ & $\underline{R}=. m$. \\
\hline 100 & 17715 & 9.782170 & 4.690065 & 4.017097 \\
\hline 95 & 16829 & 9.730858 & 4. 658574 & 4.096033 \\
\hline 90 & 15944 & 9.676838 & 4.626698 & 3.901621 \\
\hline 85 & 15058 & 9.619665 & 4. 593242 & 4.014032 \\
\hline 80 & 14172 & 9.559023 & 4. 558203 & 4.011677 \\
\hline 75 & 13286 & 9.494467 & 4. 520182 & 4.383139 \\
\hline 70 & 12401 & 9.425532 & 4.479921 & 4. 449924 \\
\hline 65 & 11515 & 9.351406 & 4.436583 & 4. 809595 \\
\hline 60 & 10629 & 9.271342 & 4.390161 & 5.046088 \\
\hline 55 & 9743 & 9.184303 & 4. 338853 & 5.412689 \\
\hline 50 & 8858 & 9.089076 & 4.281730 & 6.012647 \\
\hline 45 & 7972 & 8.983691 & 4.218701 & 5.740685 \\
\hline 40 & 7086 & 8. 865877 & 4. 148299 & 5.921705 \\
\hline 35 & 6200 & 8.732305 & 4.069269 & 6.429103 \\
\hline 30 & 5315 & $8.57828 B$ & 3.977641 & 7.069431 \\
\hline 25 & 4429 & 8. 395929 & 3. 868652 & 7.602726 \\
\hline 20 & 3543 & 8. 172728 & 3.739125 & 7.767349 \\
\hline 15 & 2657 & 7.884954 & 3.571964 & 6.718258 \\
\hline 10 & 1772 & 7.479864 & 3.332235 & 5.915089 \\
\hline 5 & 886 & 6.786717 & 2. 950274 & 4. 181003 \\
\hline
\end{tabular}

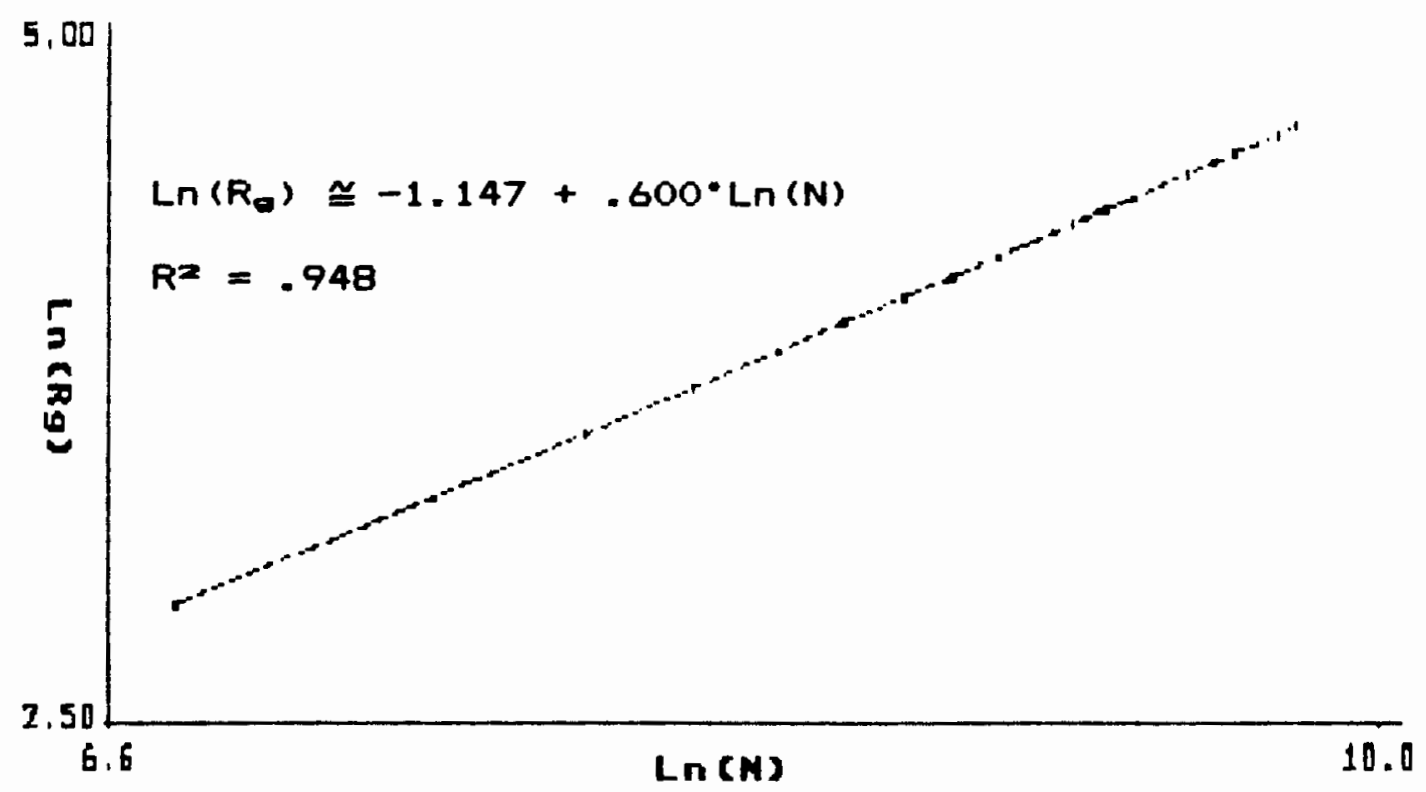

Fiqure 51. Corrected radius of gyration dependence on number of deposits for aggregate number 20 . 
in Table $V$ and the coordinates are plotted in Figure 52. The graph was analyzed using 1 inear regression and the slope of the regression line is, slope $=.571$. The correlation coefficient for the regression $i s, \quad R=.99$ and the residual variance is .028. These results yield a fractal dimension, $D_{\text {ne }}=1.75 \pm .08$. Additional analysis of the covariance of the paired points associated with the small and large forms of the aggregates was not performed because the results of the two methods of calculation were in agreement.

\section{TABLE $V$}

CORRECTED RADIUS DF GYRATION RESULTS FOR AGGREGATES NUMBEFS 1 TO 20, INCLUSIVE

AGGREGATE NUMBER

1

2

3

4

5

6

7

8

9

10

11

12

13

14

15

16

17

18

19

20

21

22

23

24

25

26
SMALL

L(N)

8. 469472

8. 249315

B. 267962

B. 438366

7.996991

8. 510973

8. 442254

8. 378850

8. 582045

8. 414496

B. 105911

B. 438581

8. 418256

8. 240913

8. 517194

8. 525558

B. 241439

8. 393839

8. 391857

8. 659385

8. 615770

8. 162231

8. 563695

B. 326517

B. 558719

8. 533068

\section{Ln (Ro)}

3.963612

3. 853862

3.850265

3.962984

3. 731075

3.994435

4.004926

3.920564

4.004637

3.925898

3. 785593

3. 952668

3.906203

3.833317

3.979296

3.983830

3.902611

3.920980

3.934570

4. 025738

4. 043975

3.839269

3. 986628

3.892380

3. 985567

4.018763

\section{LARGE}

$\begin{array}{cc}\text { L(N) } & \text { Ln }\left(R_{0}\right) \\ 9.765833 & 4.693800 \\ 9.607237 & 4.646392 \\ 9.642836 & 4.639245 \\ 9.631877 & 4.669662 \\ 9.409845 & 4.545592 \\ 9.801012 & 4.722027 \\ 9.874521 & 4.784022 \\ 9.712629 & 4.706285 \\ 9.964959 & 4.782405 \\ 9.752024 & 4.699201 \\ 9.525589 & 4.614721 \\ 9.574636 & 4.629936 \\ 9.636914 & 4.618569 \\ 9.461409 & 4.592153 \\ 9.608915 & 4.633099 \\ 9.700514 & 4.667667 \\ 9.612064 & 4.631654 \\ 9.701248 & 4.659043 \\ 9.664720 & 4.672567 \\ 9.782168 & 4.690065 \\ 9.865526 & 4.744267 \\ 9.442482 & 4.597081 \\ 9.708932 & 4.676501 \\ 9.750394 & 4.742227 \\ 9.735482 & 4.672455 \\ 9.884102 & 4.752329\end{array}$


Although time did not allow for additional analysis, an examination of the dependence that the displacement of the center of deposition has on the number of deposits could explain the concavity which was previously noticed in the graphs of $\operatorname{Ln}\left(R_{0}\right)$ vs. $\operatorname{Ln}(N)$. The previously mentioned cut offs in the regression analysis of, 0 to 6 , only excluded a relatively small number of pixels $1<2.5 \%$ of the average number of pixels, 16298). Furthermore, the displacement of the center of deposition appears to quickly attain a value comparable with the final displacement after only $5 \%$ of the total deposits. The sequence of regressions which indicated a convexity in the graphs of $\operatorname{Ln}\left(R_{0}\right) \mathrm{vs}$. $L n(N)$ (concavity in the fractal dimension) occurred over the same range of

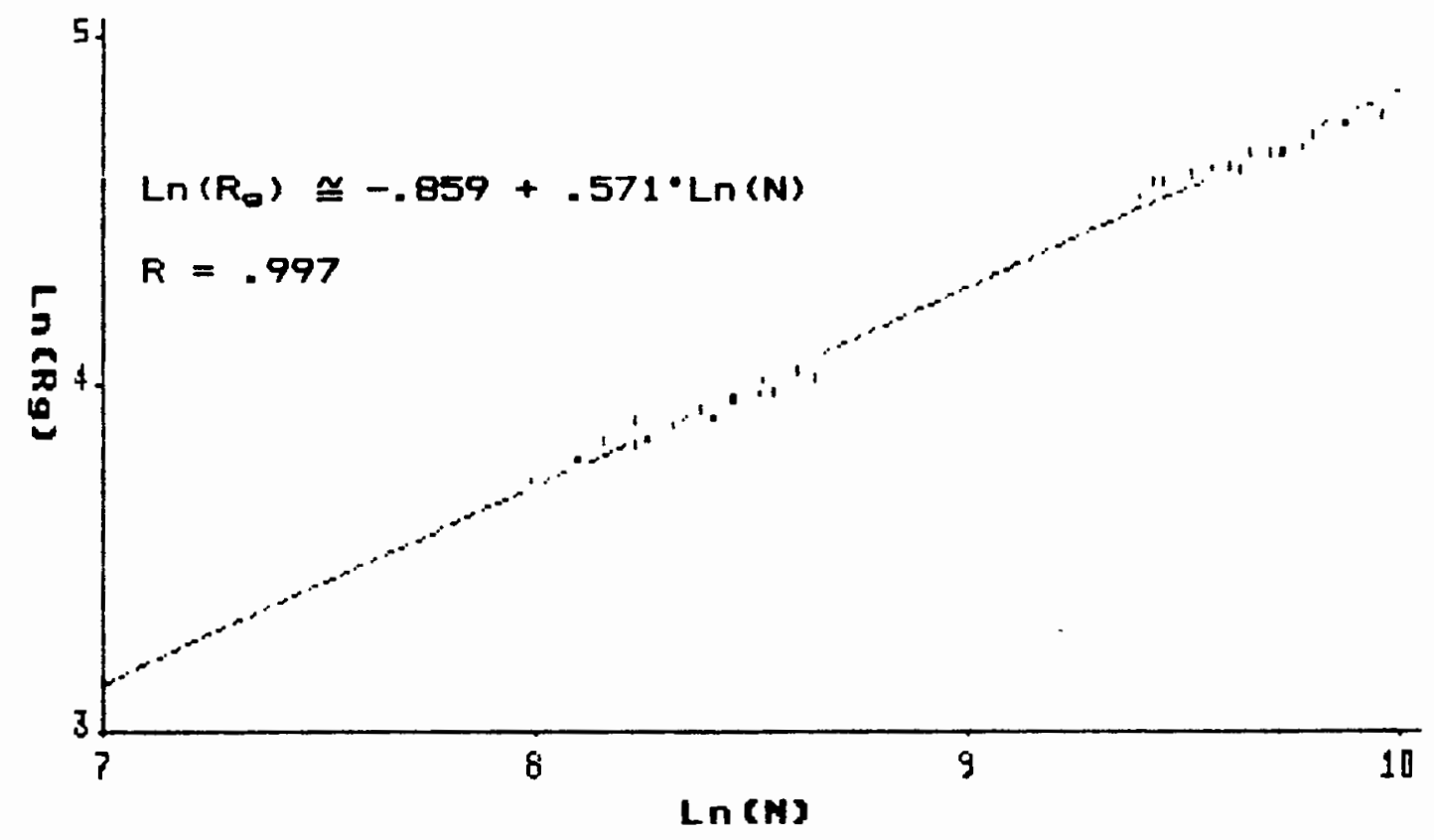

Fiqure 52. Corrected radius of gyration dependence on total number of deposits for 26 small and large aggregates. 
deposition in which the displacement was convex, evident in the data shown in Table IV for Rc.m. and $N$. This suggests that they are correlated just as the corrections to the formula for the radius of gyration would require and that the concavity may be related to the systematic error.

The estimate for the fractal dimension which is based on the average of the slopes is regarded as the most accurate. This result, $D_{m_{0}}=1.73 \pm .06$, reflecting the corrections in the radius of gyration, is approximately $3 \%$ less than the result which utilized the uncorrected radius of gyration. 
APPENDIX E

CONSIDERATIONS FOR FUFTHER WORK

In addition to those items already presented as subjects for further study, the following ideas could also provide more insight into the model.

Analysis of the effect of varying the width of the exclusion zone, or of making it more closely conform to the mean perimeter, instead of merely being concentric with the lattice origin, could provide insight into the active zone. The correlation function could also be separately evaluated over the excluded edge and the results compared to the results from the interior.

The average coordination number could be used to measure the local density and then be compared to the results of the correlation function. The sizes of the correlation windows could also be varied, although, no effect was noticed between the sizes used in this thesis to those used by Meakin.

The random walk routine could be altered with a deterministic component to simulate motion in an imposed field (Langevin equation).

The 'sticking' probability could be made to be a function of the local curvature, (Gibbs-Thompson relation) 
to realistically model solidification processes. Diffusion within the aggregate and "slumping" of the perimeter could also be investigated.

The number of jumps a random walker takes prior to deposition could be used as a psuedo-time in order to study the dynamics of growth. However, it would be necessary to adjust its values so that the velocities would not be greater for the longer jump distances in the diffusion zone. The axial center of mass could be defined along the arms of the aggregate to study the motion of the arms. Patterns and cycles of movement, independent of and also in coordination with neighboring arms could possibly be detected.

Dimensionless ratios of the step-size in the deposition zone, the size of the random walkers, and the distance of interaction with the aggregate could be formed, analogous to the Peclet number, and could be related to the fractal dimension.

The deposition probability could be found using relaxation methods, similarly, a large deposit could be bombarded many times and the number of attempted depositions could be recorded for the perimeter sites also giving the probability distribution. It is expected that the tips of the arms would have the greatest probablity. The average penetration depth could also be found.

If a color monitor were used, the age of the deposits 
could be color coded, and each color could have different diffusion and deposition properties.

The geometry of the arms could be analyzed to determine what factors might affect the ratios of the length and spacing and lengths of the side branches.

Various boundary conditions could be utilized in place of a the 'killing" circle such as reflecting or toroidial, and the geometry of the boundary could be changed to model diffusion along a channel or at a planar surface.

Finally, seeds of different geometries could be utilized, in order to investigate how persistent a sharp corner might grow, or how a cavity might be filled in. 